\title{
ELASTIC WAVES IN LAMINATED COMPOSITE PLATES FOR APPLICATION IN ULTRASONIC NONDESTRUCTIVE EVALUATION
}

\author{
BY \\ WARNAKULASOORIYA MUDIYANSELAGE \\ KARUNASENA
}

\begin{abstract}
A Thesis
Submitted to the Faculty of Graduate Studies in Partial Fulfillment of the Requirements

for the Degree of
\end{abstract}

DOCTOR OF PHILOSOPHY

Department of Civil Engineering

University of Manitoba

Winnipeg, Manitoba, Canada.

(C) April, 1992 
Bibliothèque nationale

du Canada

Direction des acquisitions et

des services bibliographiques

395 , rue Wellington

Ottawa (Ontario)
The author has granted an irrevocable non-exclusive licence allowing the National Library of Canada to reproduce, loan, distribute or sell copies of his/her thesis by any means and in any form or format, making this thesis available to interested persons.
L'auteur a accordé une licence irrévocable et non exclusive permettant à la Bibliothèque nationale du Canada de reproduire, prêter, distribuer ou vendre des copies de sa thèse de quelque manière et sous quelque forme que ce soit pour mettre des exemplaires de cette thèse à la disposition des personnes intéressées.

L'auteur conserve la propriété du droit d'auteur qui protège sa thèse. Ni la thèse ni des extraits substantiels de celle-ci ne doivent être imprimés ou autrement reproduits sans son autorisation. 
ELASTIC WAVES IN LAMINATED COMPOSITE PLATES FOR

APPLICATION IN ULTRASONIC NONDESTRUCTIVE EVALDATION

BY

WARNAKULASOOIYA MUDIYANSELAGE RARUNASENA

A Thesis submitted to the Faculty of Graduate Studies of the University of Manitoba in partial fulfillment of the requirements for the degree of

DOCTOR OF PHILOSOPHY

(C) 1992

Permission has been granted to the LIBRARY OF THE UNIVERSITY OF MANITOBA to lend or sell copies of this thesis, to the NATIONAL LIBRARY OF CANADA to microfilm this thesis and to lend or sell copies of the film, and UNIVERSTTY MICROFILMS to publish an abstract of this thesis.

The author reserves other publication rights, and neither the thesis nor extensive extracts from it may be printed or otherwise reproduced without the author's permission. 
To my family members 


\begin{abstract}
Propagation and scattering of free guided waves in laminated composite plates are investigated with particular reference to ultrasonic nondestructive evaluation (NDE) of material properties and flaws.

A stiffness method and an analytical method have been developed to study wave propagation in laminated composite plates. Using these methods, the effects of interface layers, anisotropy, and layering on dispersion characteristics of free guided waves are investigated. It is shown that ultrasonic NDE techniques can be used to characterise material properties of these plates.

As a special case of a wave scattering problem, the problem of reflection of waves normally incident upon the free edge of a laminated composite plate has been investigated in detail using least-squares and variational methods. A wave function expansion procedure has been used in developing least-squares and variational methods for this problem. The end resonance for a homogeneous fiber-reinforced composite plate is reported.

A hybrid method is presented for analyzing scattering of waves by the flaws in laminated composite plates. The modeling is achieved by dividing the domain of the plate into two regions: an interior region that consists of the flaws and a finite region of the plate around the flaw; and an unbounded exterior region. The hybrid method combines a finite element formulation in the interior region with a wave function representation in the exterior region. The method is illustrated through solving the problems of scattering by a symmetric normal edge crack and a centrally located normal matrix crack that grows into delamination. The technique presented has potential applications in ultrsonic NDE.
\end{abstract}




\section{Acknowledgements}

The author wishes to express his deepest gratitude to his thesis advisor, Prof. A.H. Shah, for suggesting the research topic and providing continued expert guidance up to the completion of this study. Encouragement and support received from Prof. Shah throughout this work is greatly appreciated.

The author is extremely thankful to Prof. R.K.N.D. Rajapakse and Prof. L. Shafai for helpful suggestions and serving in the advisory committee. Sincere thanks are extended to Prof. S.K. Datta for his fruitful suggestions and guidance.

The author deeply appreciates the support and encouragement of his colleagues, Dr. Y.M. Desai and Dr. U.G.A. Puswewala, during graduate work at The University of Manitoba. A word of thanks is also extended to the all of authors friends, and specially to Dr. R. Paskaramoorthy, Dr. R.L. Bratton, Dr. R. Zhang, Miss. G. Abeydeera, Mr. N. Rattanawangcharoen, and Mr. Y. Wang for their help. Grateful acknowledgements are also due to the staff of the Computer Services of The University of Manitoba for their help in providing computer facilities.

Financial support in the form of a research assistantship from the NSERC research grant of Prof. Shah, a graduate fellowship from The University of Manitoba, and a teaching assitantship from the Department of Civil Engineering enabled the author to pursue his graduate studies. They are kindly acknowledged.

The influence of Prof. P. Karasudhi of The Asian Institute of Technology in developing author's interest in research is greatly appreciated.

The author wishes to convey sincere thanks to his wife for her understanding and moral support during the course of this study. Special thanks are due to the author's little son, Sajith, for his patience when he missed cuddling by daddy. Moral 
support received from author's parents, brothers and sister are kindly appreciated.

Finally, the author respectfully dedicate this thesis to his parents whose unfailing love and inspiration helped him to achieve this stage of his career.

April, 1992

Warnakulasooriya M. Karunasena

Winnipeg, Canada. 


\section{Table of Contents}

Title

Page No.

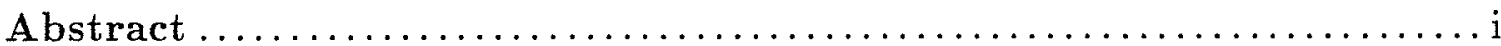

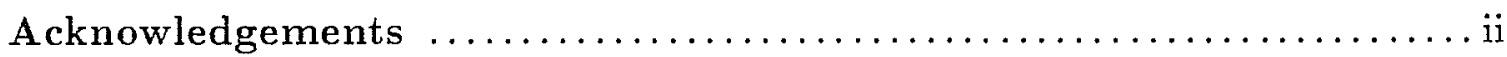

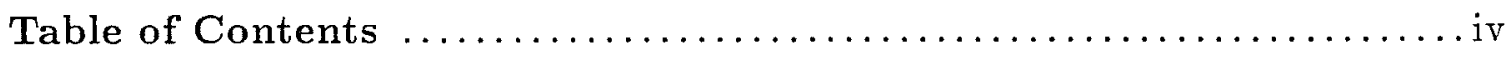

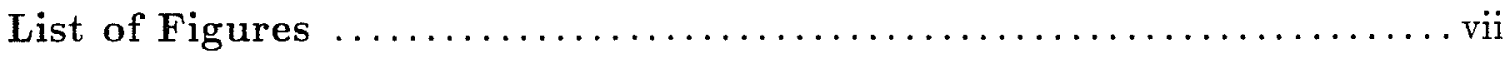

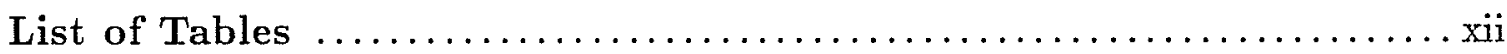

1. Introduction 1

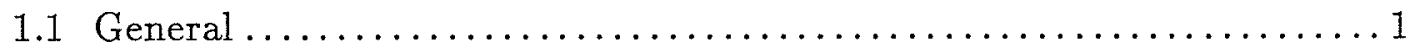

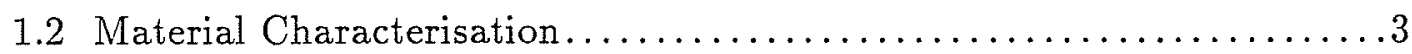

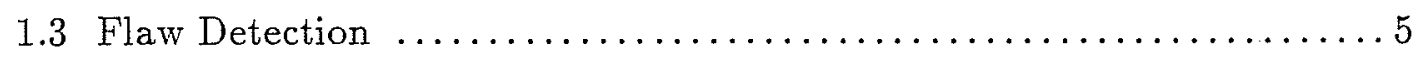

1.4 An Overview of the Present Study $\ldots \ldots \ldots \ldots \ldots \ldots \ldots \ldots \ldots \ldots \ldots$

1.4.1 Guided Wave Propagation $\ldots \ldots \ldots \ldots \ldots \ldots \ldots \ldots \ldots \ldots$

1.4.2 Free End Reflection .......................... 10

1.4.3 Wave Scattering by Cracks and Delaminations............11

1.5 Organisation of the Thesis $\ldots \ldots \ldots \ldots \ldots \ldots \ldots \ldots \ldots \ldots \ldots \ldots \ldots \ldots \ldots \ldots \ldots \ldots \ldots$

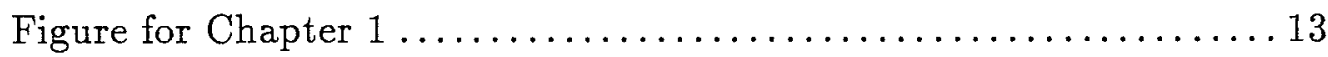

2. Wave Propagation in Laminated Composite Plates 14

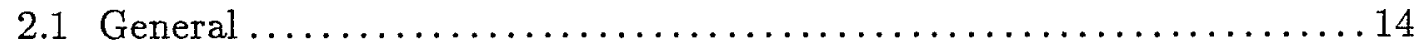

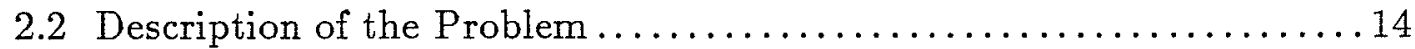

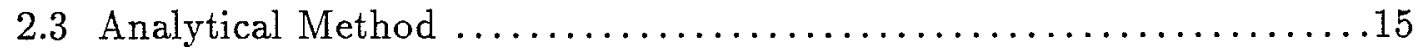

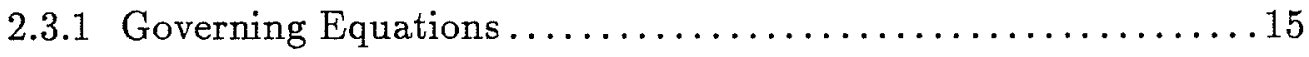

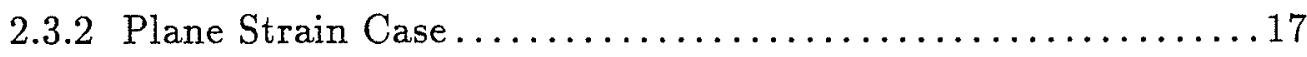

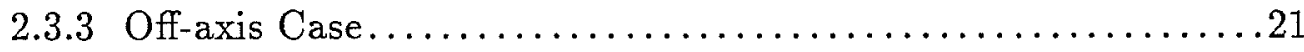

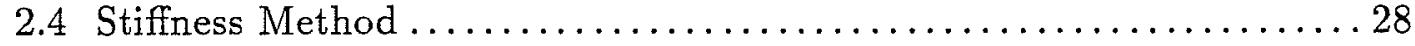

2.5 Roots of Dispersion Equation ............................ 33

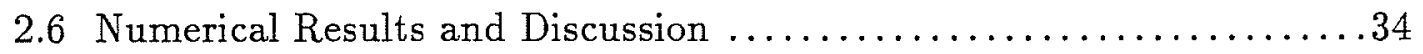

2.6.1 Accuracy of Stiffness Method I .................... 35

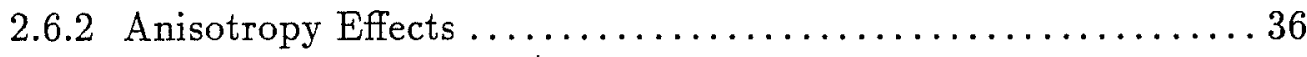

2.6.3 Interface Layer Effects . ......................... 37

2.6.4 Layering Effects............................. 38 


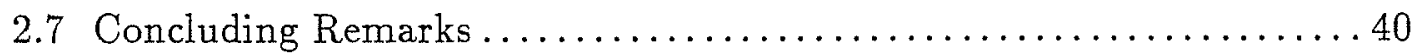

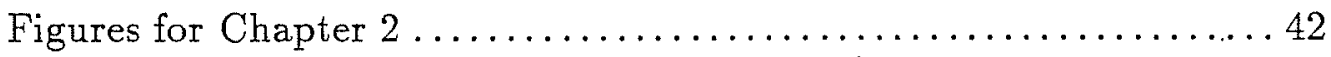

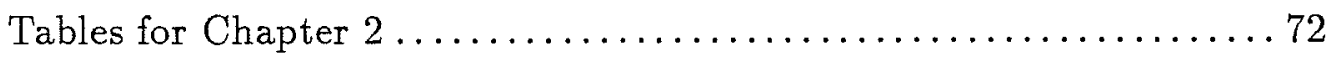

3. Reflection of Waves at the Free Edge of a Laminated

Composite Plate $\quad 74$

3.1 General ............................................. 74

3.2 Description of the Problem ............................. 75

3.3 Wave Functions ................................... 75

3.4 Reflection Coefficients ................................ 77

3.5 Energy Flux....................................... 81

3.6 Numerical Results and Discussion $\ldots \ldots \ldots \ldots \ldots \ldots \ldots \ldots \ldots \ldots . \ldots . \ldots \ldots$

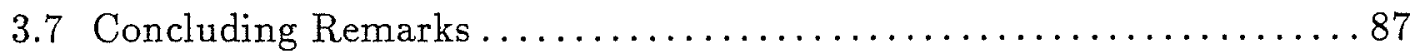

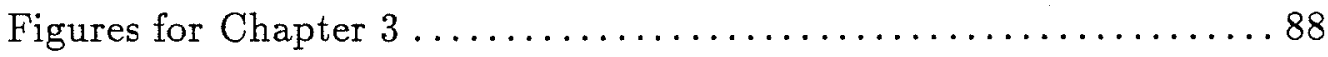

Tables for Chapter $3 \ldots \ldots \ldots \ldots \ldots \ldots \ldots \ldots \ldots \ldots \ldots \ldots \ldots \ldots$

4. Wave Scattering by Cracks and Delaminations in Laminated

Composite Plates $\quad 99$

4.1 General ............................................... 99

4.2 Description of the Problem.............................. 100

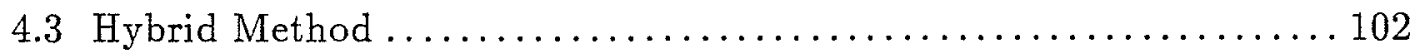

4.4 Plane Strain Case ................................... 103

4.4.1 Wave Functions for Exterior Regions ................. 103

4.4.2 Finite Element Model of Interior Region................. 105

4.4 .3 Global Solution ............................... 109

4.4.4 Energy Conservation............................ 111

4.4.5 Reciprocity Relations ............................ 112

4.5 General Case....................................... 113

4.5.1 Wave Functions for Exterior Regions .................. 114

4.5.2 Finite Element Model of Interior Region................ 118

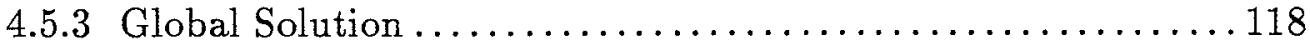

4.5.4 Energy Conservation and Reciprocity Relations............119

4.6 Numerical Results and Discussion ........................ 119

4.6.1 Plane Strain Case.............................. 122 
4.6.1.1 Normal Edge Crack......................... 122

4.6.1.2 Normal Central Crack Extending to Delamination.... 124

4.6.2 General Case ............................... 127

4.7 Concluding Remarks ............................... 129

Figures for Chapter $4 \ldots \ldots \ldots \ldots \ldots \ldots \ldots \ldots \ldots \ldots \ldots \ldots \ldots \ldots \ldots$

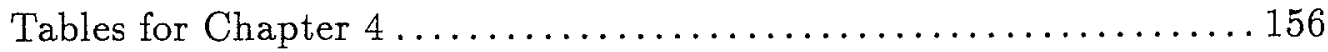

5. Conclusions, and Recommendations for Future Work 158

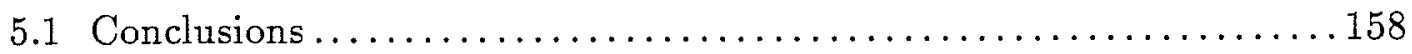

5.2 Recommendations for Future Work ....................... 160

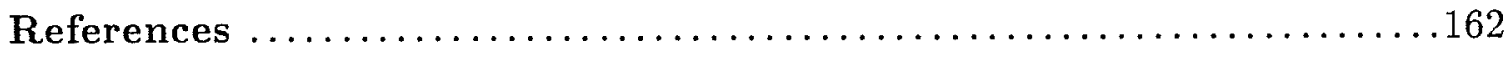

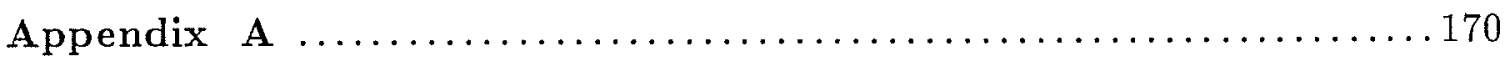

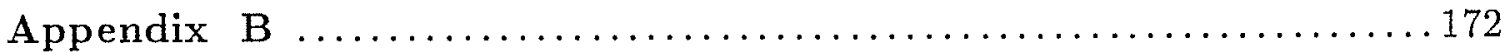

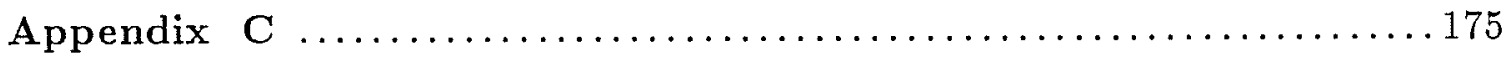

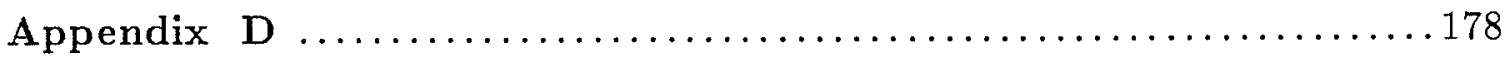

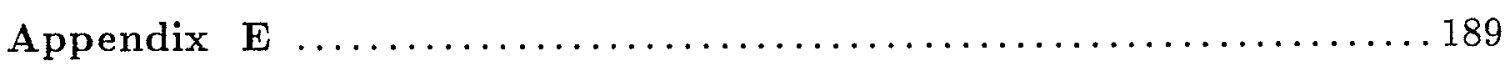

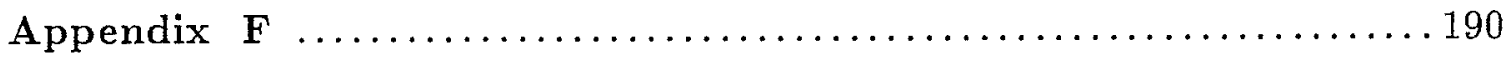




\section{List of Figures}

Figure

Title

Page

1.1 Schematic diagram of a typical ultrasonic flaw detector. ............ 13

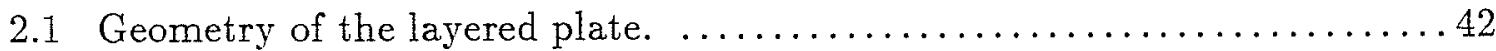

2.2 Comparison of the results for the dispersion curves from the stiffness method I with analytical results for a homogeneous graphite-epoxy plate, for propagation in the $0^{\circ}$ direction. $\ldots \ldots \ldots \ldots \ldots \ldots \ldots \ldots \ldots \ldots \ldots \ldots \ldots$

2.3 Dispersion curves for a 3 -layer cross-ply $\left(0^{\circ} / 90^{\circ} / 0^{\circ}\right)$ graphite-epoxy plate, for propagation in the $45^{\circ}$ direction. . . . . . . . . . . . . . . . . 44

2.4 Dispersion curves for a 35 -layer cross-ply $\left(0^{\circ} / 90^{\circ} / 0^{\circ} / \cdots \cdots / 0^{\circ} / 90^{\circ} / 0^{\circ}\right)$ graphite-epoxy plate, for propagation in the $45^{\circ}$ direction. . . . . . . . . 45

2.5 Dispersion curves for a homogeneous graphite-epoxy plate, for propagation in the:
(a) $5^{\circ}$ direction,
(b) $30^{\circ}$ direction,
(c) $60^{\circ}$ direction,
(d) $85^{\circ}$ direction.

2.6 Dispersion curves for a 3-layer angle-ply $\left(0^{\circ} / 2 \alpha / 0^{\circ}\right)$ graphite-epoxy plate, for propagation in the $\alpha$ direction:
(a) $\alpha=5^{\circ}$,
(b) $\alpha=30^{\circ}$,
(c) $\alpha=60^{\circ}$,
(d) $\alpha=85^{\circ}$.

2.7 Dispersion of waves in a 19 -layer cross-ply $\left(0^{\circ} / 90^{\circ} / 0^{\circ} / \cdots \cdots / 0^{\circ} / 90^{\circ} / 0^{\circ}\right)$ graphite-epoxy plate, for propagation in the $0^{\circ}$ direction: $\ldots \ldots \ldots \ldots \ldots 50$

2.8 Dispersion of waves in a 19 -layer cross-ply $\left(0^{\circ} / 90^{\circ} / 0^{\circ} / \cdots \cdots / 0^{\circ} / 90^{\circ} / 0^{\circ}\right)$ graphite-epoxy plate, for propagation in the $90^{\circ}$ direction: $\ldots \ldots \ldots \ldots \ldots 1$

2.9 Dispersion of waves in a 19-layer cross-ply $\left(0^{\circ} / 90^{\circ} / 0^{\circ} / \cdots \cdots / 0^{\circ} / 90^{\circ} / 0^{\circ}\right)$ graphite-epoxy plate with interface layers between $0^{\circ}$ and $90^{\circ}$ layers: The ratios of the thickness and densities of a layer and an interface are 10 and 1.5 , respectively; Propagation is in the $0^{\circ}$ direction. ..........52

2.10 Dispersion of waves in a 19 -layer cross-ply $\left(0^{\circ} / 90^{\circ} / 0^{\circ} / \cdots \cdots / 0^{\circ} / 90^{\circ} / 0^{\circ}\right)$ graphite-epoxy plate with interface layers between $0^{\circ}$ and $90^{\circ}$ layers: The ratios of the thickness and densities of a layer and an interface are 10 and 1.5, respectively; Propagation is in the $90^{\circ}$ direction. ..........53

2.11 Same as in Figure 2.9, but the density ratio is $2 / 3 . \ldots \ldots \ldots \ldots \ldots \ldots . .54$ 
2.12 Same as in Figure 2.10, but the density ratio is $2 / 3 . \ldots \ldots \ldots \ldots \ldots \ldots 55$

2.13 Same as in Figure 2.9, but the thickness ratio is $5 . \ldots \ldots \ldots \ldots \ldots \ldots 56$

2.14 Same as in Figure 2.9, but the thickness ratio is $10 / 3 \ldots \ldots \ldots \ldots \ldots \ldots 7$

2.15 Dispersion of waves in a 3-layer cross-ply $\left(0^{\circ} / 90^{\circ} / 0^{\circ}\right)$ graphite-epoxy

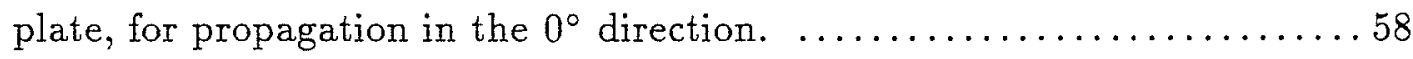

2.16 Dispersion of waves in a 3-layer cross-ply $\left(0^{\circ} / 90^{\circ} / 0^{\circ}\right)$ graphite-epoxy plate, for propagation in the $90^{\circ}$ direction. ......................5 59

2.17 Dispersion of waves in a 21 -layer cross-ply $\left(0^{\circ} / 90^{\circ} / 0^{\circ} / \cdots \cdots / 0^{\circ} / 90^{\circ} / 0^{\circ}\right)$ graphite-epoxy plate, for propagation in the $0^{\circ}$ direction. $\ldots \ldots \ldots \ldots \ldots 60$

2.18 Dispersion of waves in a 21 -layer cross-ply $\left(0^{\circ} / 90^{\circ} / 0^{\circ} / \cdots \cdots / 0^{\circ} / 90^{\circ} / 0^{\circ}\right)$ graphite-epoxy plate, for propagation in the $90^{\circ}$ direction. ............61

2.19 Dispersion of waves in a 27 -layer cross-ply $\left(0^{\circ} / 90^{\circ} / 0^{\circ} / \cdots \cdots / 0^{\circ} / 90^{\circ} / 0^{\circ}\right)$ graphite-epoxy plate, for propagation in the $0^{\circ}$ direction. ............662

2.20 Dispersion of waves in a 27 -layer cross-ply $\left(0^{\circ} / 90^{\circ} / 0^{\circ} / \cdots \cdots / 0^{\circ} / 90^{\circ} / 0^{\circ}\right)$ graphite-epoxy plate, for propagation in the $90^{\circ}$ direction. . ...........66 63

2.21 Dispersion of waves in a 35-layer cross-ply $\left(0^{\circ} / 90^{\circ} / 0^{\circ} /\right.$ cdots $\left.\cdots / 0^{\circ} / 90^{\circ} / 0^{\circ}\right)$ graphite-epoxy plate, for propagation in the $0^{\circ}$ direction. . ............64

2.22 Dispersion of waves in a 35-layer cross-ply $\left(0^{\circ} / 90^{\circ} / 0^{\circ} / \cdots \cdots / 0^{\circ} / 90^{\circ} / 0^{\circ}\right)$ graphite-epoxy plate, for propagation in the $90^{\circ}$ direction. ............65

2.23 Prediction of dispersion from the effective modulus model,

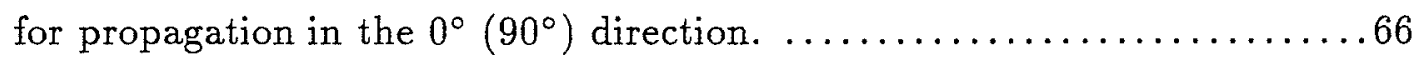

2.24 Dispersion of waves in a 35-layer cross-ply $\left(0^{\circ} / 90^{\circ} / 0^{\circ} / \cdots \cdots / 0^{\circ} / 90^{\circ} / 0^{\circ}\right)$ graphite-epoxy plate, for propagation in the $22.5^{\circ}$ direction. ..........67 67

2.25 Prediction of dispersion from the effective modulus model, for propagation in the $22.5^{\circ}$ direction. $\ldots \ldots \ldots \ldots \ldots \ldots \ldots \ldots \ldots \ldots 6$

2.26 Dispersion of waves in a 35-layer cross-ply $\left(0^{\circ} / 90^{\circ} / 0^{\circ} / \cdots \cdots / 0^{\circ} / 90^{\circ} / 0^{\circ}\right)$ graphite-epoxy plate, for propagation in the $45^{\circ}$ direction. . ...........69 69

2.27 Prediction of dispersion from the effective modulus model,

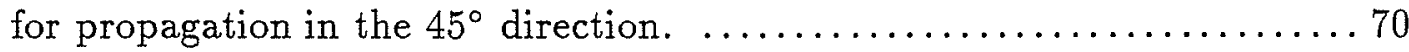

2.28 Dispersion of waves in a 35-layer cross-ply $\left(0^{\circ} / 90^{\circ} / 0^{\circ} / \cdots \cdots / 0^{\circ} / 90^{\circ} / 0^{\circ}\right)$ graphite-epoxy plate, for propagation in the $67.5^{\circ}$ direction. ..........71

3.1 Geometry of the semi-infinite layered plate. $\ldots \ldots \ldots \ldots \ldots \ldots \ldots \ldots . \ldots 8$ 
3.2 Real branches of the frequency spectrum

(a) Homogeneous graphite-epoxy plate,

(b) 35-layer cross-ply $\left(90^{\circ} / 0^{\circ} / \cdots / 90^{\circ} / 0^{\circ} / 90^{\circ} / \cdots / 0^{\circ} / 90^{\circ}\right)$

graphite-epoxy plate.

3.3 The proportions of energy $E_{1 n}$ vs $\Omega$, for the homogeneous isotropic plate, due to first symmetric incident mode $(\nu=0.25) . \ldots \ldots \ldots \ldots \ldots \ldots$

3.4 The proportions of energy $E_{1 n}$ vs $\Omega$, for the homogeneous graphite-epoxy plate, due to first symmetric incident mode. . ........ 91

3.5 The reflection coefficient $\left|R_{1 n}\right|$ vs $\Omega$, for the homogeneous graphite-epoxy plate, due to first symmetric incident mode
(a) $n=1$,
(b) $n=2$.

3.6 The proportions of energy $E_{1 n}$ vs $\Omega$, for the 35-layer graphite-epoxy plate, due to first symmetric incident mode. . . . . . . . . . . . . . . 93

3.7 The proportions of energy $E_{1 n}$ vs $\Omega$, for the 35-layer graphite-epoxy plate, due to first antisymmetric incident mode. ................ 94

3.8 The reflection coefficient $\left|R_{11}\right|$ vs $\Omega$, for the 35 -layer graphite-epoxy plate,

(a) due to first symmetric incident mode,

(b) due to first antisymmetric incident mode. 95

4.1 Geometry of the layered plate

(a) Elevation showing subdivision of layers,

(b) Plan view of a typical $(i$-th) sublayer showing orientation of fibers and wave normals.

4.2 Geometry of flaws and details of regions

(a) Normal edge crack in á plate

(b) Centrally located normal matrix crack extending to delamination

(shown by solid and dashed lines) in an 8-layer cross-ply

$\left(0^{\circ} / 90^{\circ} / 0^{\circ} / 90^{\circ} / 90^{\circ} / 0^{\circ} / 90^{\circ} / 0^{\circ}\right)$ plate

(c) A typical finite element mesh near the crack tip.

4.3 $R_{11}$ vs normalized crack length at $\Omega=\pi / 2$ for example 1 , due to first symmetric incident mode (plane strain case).

$4.4 R_{11}$ vs normalized crack length at $\Omega=2.0$ for example 2 , due to first symmetric incident mode (plane strain case). 
4.5 $R_{1 n}$ vs normalized crack length at $\Omega=4.0$ for example 2 , due to first symmetric incident mode (plane strain case). . . . . . . . . . . . 136

4.6 $R_{11}$ vs normalized crack length at $\Omega=1.2$ for example 3 , due to first symmetric incident mode (plane strain case). .....................

4.7 $R_{11}$ vs normalized crack length at $\Omega=2.5$ for example 3 , due to first symmetric incident mode (plane strain case) . .................... 138

$4.8 R_{11}$ vs normalized crack length at $\Omega=1.2$ for example 3 , due to first antisymmetric incident mode (plane strain case) . .................. 139

$4.9 R_{1 n}$ vs normalized crack length at $\Omega=2.5$ for example 3 , due to first antisymmetric incident mode (plane strain case).

4.10 Real branches of the frequency spectrum for an 8-layer cross-ply $\left(0^{\circ} / 90^{\circ} / 0^{\circ} / 90^{\circ} / 90^{\circ} / 0^{\circ} / 90^{\circ} / 0^{\circ}\right)$ graphite-epoxy plate (plane strain case).

4.11 Variation of $\left|T_{1 n}\right|$ and $\left|R_{1 n}\right|$ with normalized crack length at $\Omega=4.0$ for example 4 , due to first symmetric incident mode (plane strain case):
(a) $\left|T_{1 n}\right|$ vs $b / b_{1}$,
(b) $\left|R_{1 n}\right|$ vs $b / b_{1}$

4.12 Variation of $\left|T_{2 n}\right|$ and $\left|R_{2 n}\right|$ with normalized crack length at $\Omega=4.0$ for example 4 , due to second symmetric incident mode (plane strain case):
(a) $\left|T_{2 n}\right|$ vs $b / b_{1}$
(b) $\left|R_{2 n}\right|$ vs $b / b_{1}$.

4.13 Variation of $\left|T_{3 n}\right|$ and $\left|R_{3 n}\right|$ with normalized crack length at $\Omega=4.0$ for example 4 , due to third symmetric incident mode (plane strain case):
(a) $\left|T_{3 n}\right|$ vs $b / b_{1}$,
(b) $\left|R_{3 n}\right|$ vs $b / b_{1}$

144

4.14 Variation of $\left|T_{1 n}\right|$ and $\left|R_{1 n}\right|$ with normalized crack length at $\Omega=4.0$ for example 4 , due to first antisymmetric incident mode (plane strain case):

$\begin{array}{ll}\text { (a) }\left|T_{1 n}\right| \text { vs } b / b_{1}, & \text { (b) }\left|R_{1 n}\right| \text { vs } b / b_{1}\end{array}$ .145

4.15 Variation of $\left|T_{2 n}\right|$ and $\left|R_{2 n}\right|$ with normalized crack length at $\Omega=4.0$ for example 4 , due to second antisymmetric incident mode (plane strain case): 
(a) $\left|T_{2 n}\right|$ vs $b / b_{1}$,

(b) $\left|R_{2 n}\right|$ vs $b / b_{1}$.

4.16 Variation of $\left|T_{3 n}\right|$ and $\left|R_{3 n}\right|$ with normalized crack length

at $\Omega=4.0$ for example 4 , due to third antisymmetric incident mode (plane strain case):

(a) $\left|T_{3 n}\right|$ vs $b / b_{1}, \quad$ (b) $\left|R_{3 n}\right|$ vs $b / b_{1}$

4.17 Variation of $\left|T_{11}\right|$ and $\left|R_{11}\right|$ with $\Omega$ for example 4, due to first symmetric incident mode, when $b / b_{1}=1.0$ (plane strain case).

4.18 Variation of $\left|T_{11}\right|$ and $\left|R_{11}\right|$ with $\Omega$ for example 4 , due to first symmetric incident mode, when $b / b_{1}=1.5$ (plane strain case).

4.19 Variation of $\left|T_{1 n}\right|$ and $\left|R_{1 n}\right|$ with $\Omega$ for example 4 , due to first symmetric incident mode, when $b / b_{1}=1.0$ (plane strain case):
(a) $\left|T_{1 n}\right|$ vs $\Omega$,
(b) $\left|R_{1 n}\right|$ vs $\Omega$.

4.20 Variation of $\left|T_{1 n}\right|$ and $\left|R_{1 n}\right|$ with $\Omega$ for example 4, due to first symmetric incident mode, when $b / b_{1}=1.5$ (plane strain case):
(a) $\left|T_{1 n}\right|$ vs $\Omega$,
(b) $\left|R_{1 n}\right|$ vs $\Omega$.

4.21 Variation of $\left|T_{2 n}\right|$ and $\left|R_{2 n}\right|$ with $\Omega$ for example 4, due to second symmetric incident mode, when $b / b_{1}=1.0$ (plane strain case):
(a) $\left|T_{2 n}\right|$ vs $\Omega$,
(b) $\left|R_{2 n}\right|$ vs $\Omega$.

4.22 Variation of $\left|T_{2 n}\right|$ and $\left|R_{2 n}\right|$ with $\Omega$ for example 4, due to second symmetric incident mode, when $b / b_{1}=1.5$ (plane strain case):
(a) $\left|T_{2 n}\right|$ vs $\Omega$,
(b) $\left|R_{2 n}\right|$ vs $\Omega$.

4.23 Variation of $\left|T_{3 n}\right|$ and $\left|R_{3 n}\right|$ with $\Omega$ for example 4 , due to third symmetric incident mode, when $b / b_{1}=1.0$ (plane strain case):

(a) $\left|T_{3 n}\right|$ vs $\Omega$,

(b) $\left|R_{3 n}\right|$ vs $\Omega$. 154

4.24 Variation of $\left|T_{3 n}\right|$ and $\left|R_{3 n}\right|$ with $\Omega$ for example 4 , due to third symmetric incident mode, when $b / b_{1}=1.5$ (plane strain case):
(a) $\left|T_{3 n}\right|$ vs $\Omega$,
(b) $\left|R_{3 n}\right|$ vs $\Omega$. 


\section{List of Tables}

Table

Title

Page

2.1 Comparison among results for frequencies from stiffness method I, stiffness method II, and analytical method, for propagation in the $45^{\circ}$ direction in a homogeneous graphite-epoxy plate.

2.2 Comparison among results for frequencies from stiffness method I, stiffness method II, and analytical method, for propagation in the $45^{\circ}$ direction in a 3 -layer cross-ply $\left(0^{\circ} / 90^{\circ} / 0^{\circ}\right)$ graphite-epoxy plate. $\ldots 73$

3.1 Elastic stiffnesses of $0^{\circ}$ and $90^{\circ}$ graphite-epoxy laminae. . . . . . . . 96

3.2 Variation of percentage error $\varepsilon$ in energy balance and reflection coefficient $\left|R_{11}\right|$ with number of modes $M$, for homogeneous

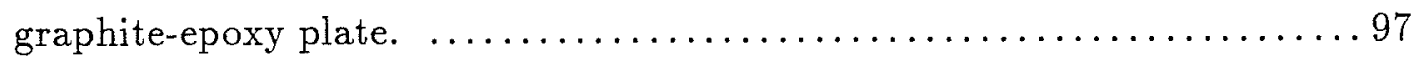

3.3 Variation of percentage error $\varepsilon$ in energy balance and reflection coefficient $\left|R_{11}\right|$ with number of modes $M$, for 35-layer

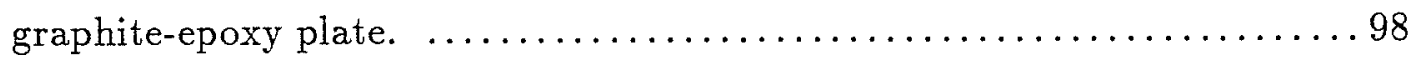

4.1 Numerical reults for scattering problem in example 2 at $\Omega=2.0$ (general case).

4.2 Numerical reults for scattering problem in example 5 at $\Omega=4.0$ and $a / H=0.5$ (general case). 157 


\section{Ch a p t e r 1}

\section{Introduction}

\subsection{General}

Composite materials are ushering in a new era for material science and engineering due to their low density, increased service life, and high performance not obtainable with conventional engineering materials. A composite material is a combination of two or more constituents which results in a substance having a potentially new range of performance characteristics. There are two basic types of composites, namely, particle-reinforced composites and fiber-reinforced composites. Particle reinforced composites are elemental or alloy matrices within which fine particles of such materials as $\mathrm{Al}_{2} \mathrm{O}_{3}, \mathrm{TnO}_{2}, \mathrm{Cr}_{2} \mathrm{O}_{3}, \mathrm{SiO}_{2}, \mathrm{SiC}$ are dispersed. Fiber-reinforced composites are elemental, alloy or polymeric matrices strengthened by random or oriented fibers (filaments). The contents of this thesis will be mainly directed towards the oriented fiber-reinforced composites, although some of the principles to be discussed will have general applicability.

In fiber-reinforced composites, the role of the fibers is to impart the stiffness and strength characteristics of the fibers by being able to carry a major part of the load in the direction of the fibers. The fibers may be organic or metalic. Examples of the commonly used fibers are glass, graphite, boron, and aramid (Kevlar). The diameters of these fibers are typically in the range of $0.008 \mathrm{~mm}$ to $0.15 \mathrm{~mm}$. Generally the fibers are 10 to 100 times stronger than the matrix. The role of the matrix is: to maintain alignment of the fibers and increase structural stiffness; to provide adequate transeverse properties perpendicular to the fibers; to act as a load transfer medium for discontinuous or broken fibers; and to protect the fibers from damage, 
mutual abrasion and environmental degradation. The matrix may be organic or metalic. Some examples of the matrices are epoxy, polyester, and silicon.

The use of composite materials began with the development and use of fiberreinforced plastic composites in the 1940s, when the engineers were looking for alternatives to expensive and scarce steels or alloys. Since then, the military and a variety of other industries (such as aerospace, marine, automobile, construction) have developed numerous applications for composites. Many of these applications use composites in the form of plates. In the manufacturing process, the continuous fibers are laid parallel to each other, and then emersed into the matrix to form a single layer or lamina. Some applications use composites in the form of single lamina, which are commonly referred to as homogeneous or uniaxial fiber-reinforced composite plates. For many other applications, the single layers are hand or machine bound together with thin resin or plastic bond layers to produce what is known as laminated composite plates. The present investigation is concerned with such plates. Almost any number of single layers can be laminated, and the fibers in adjacent layers can be oriented in differnt directions to suit with the structural requirements. The composite plates have several advantages over the plates made of conventional materials. They offer superior strength to weight ratio, and are corrosion-resistant, thermally insulating, non-magnetic, and dielectric. Another advantage of using these structural members is the possibilty of tailoring the properties of the fibers for a particular use. In order to use these stuctural members at their fullest potential, several problems related to their strength behaviour have to be solved. The strength of these plates is dependent on the elastic properties of the composite material, and in addition the presence of flaws or defects in the composite material has a great influence on the strength. 


\subsection{Material Characterisation}

Ultrasonic waves provide an efficient means of characterising effective elastic properties of a composite or a heterogeneous material. Several studies (Datta, 1977; Read and Ledbetter, 1977; Berryman, 1980a, 1980b; Datta and Ledbetter, 1983; Kinra et al., 1980; Datta et al., 1984; Ledbetter and Datta, 1984; Varadan et al., 1985; Ledbetter and Datta, 1986; Datta and Ledbetter, 1987; Ledbetter et al., 1989) show that, for long wave lengths one can predict the effective wave speeds of plane waves through such a material. At long wave lengths wave speeds are nondispersive, and hence provide the values for effective elastic properties of the composite material.

In laminated composite plates, the material properties of interface bond layers and laminae are often quite different in bulk from those of manufactured specimens, due to changes induced by the curing process. Thus it is important to nondestructively characterise these materials in situ. Several ultrasonic techniques are currently available for nondestructive evaluation (NDE) of plate-like specimens. Early techniques used body waves. New techniques employing guided waves are under development. There are several advantages in using guided waves in the NDE. First, their dispersive and multimodal nature can be used to generate a large number of data points in a given frequency range, compared with only a few in the case of body waves. Second, the velocity of guided waves is very sensitive to the material properties of the plate. Finally, the velocity of guided waves can be very accurately measured as a function of frequency.

In order to interpret ultrasonic test results, it is neccessary to have theoretical predictions for dispersion characteristics of guided waves as fuctions of the properties to be characterised. Comparision of test results with theoretical predictions leads 
to determining unknown properties. Dispersion charateristics of guided waves in an undamaged laminated composite plate are influenced by many factors, the most important of which are: interfaces, fiber orientation in each lamina, number of layers, and effective material properties of each lamina.

Using elasticity equations, dispersion relation for a homogeneous isotropic plate has been extensively studied by Mindlin (1960). Dispersion characteristics of two- or three-layer istropic plates have been analytically investigated by Yu (1960), Jones (1964), and Lee and Chang (1979). Frequency spectrum for monoclinic crystal plates has been investigated by Kaul and Mindlin (1962). Recently, Nayfeh and Chimenti (1989) studied propagation of guided waves in an anisotropic homogeneous plate in detail. This study contains a comprehensive survey of the literature on guided wave propagation in homogeneous anisotropic plates. Baylis and Green (1986a, 1986b) and Baylis (1988) investigated analytical dispersion equations for two- or three-layer transversely isotropic plates.

Several approximate theories have been proposed to derive the dispersion relations in the form of generalized eigenproblems. The most common ones are plate theories. A list of references on numerous refined plate theories for homogeneous or laminated media consisting of isotropic or anisotropic materials can be found in $\mathrm{Ka}$ pania and Raciti (1989), and Librescu and Reddy (1989). However, plate theories are cumbersome to use and they do not provide very accurate eigenvalues for wave velocities. Theories which yield accurate eigenvalues and are computationally very convenient to use are the theories derived through the stiffness method of analysis. Dong and his co-workers (Dong and Nelson, 1972; Dong and Pauley, 1978; Dong and Huang, 1985) presented a stiffness method of analysis to study wave propagation in laminated anisotropic plates. They discretized the plate in thickness direction with subdivision into mathematical sublayers and used quadratic interpolation polyno- 
mials that involve only the displacements at the interfaces between sublayers and at the middle of the sublayers as the generalized coordinates. Since only displacement continuity is maintained, this method is not very effective for obtaining accurate wave velocities at high frequencies. Recently, Datta et al. (1988) presented an approximate stiffness method applicable to a layered anisotropic plate with arbitrary number of layers. In this method, the continuity of both displacements and tractions are maintained. In their analysis, they considered only plane strain and antiplane strain propagation problems. A comprehensive review of previous work on guided wave propagation in the presence of thin interface bond layers can be found in Datta et al. (1990b). In most of these studies, the bond layer is approximated as a massless spring or a fluid layer that allows jump in the displacement keeping traction continuous. Attempts at using this spring or slip model to characterise bond layer have shown their applicability to a limited class of bonds. For further references on previous studies of guided wave dispersion, the reader is referred to the edited volume of Datta et al. (1990a). However, in none of the above studies a systematic study of effects of interface bond layers, anisotropy, and layering on the dispersion characteristics of guided waves in a laminated composite plate has been reported.

In the studies mentioned above, attention was focussed on free guided waves. It is noted here that the effect of fluid loading on ultrasonic guided waves has also been investigated by several authors. References to these can be found in the works by Rokhlin and Marom (1986), Nayfeh and Chimenti (1988), Pilarski and Rose (1988), Chimenti and Nayfeh (1990), and Mal et al. (1990).

\subsection{Flaw Detection}

Presence of flaws is a major controlling factor of strength of a laminated com- 
posite plate. These plates have unique failure mechanisms. For example, the bond between the layers may fail causing delamination or debonding. Similarly, the matrix can have surface cracks. A most likely failure in a cross-ply laminated plate is a normal matrix crack initiated within an inner layer, extending to the adjacent layers and then growing into delamination. If the plate is a part of a structural system that cannot be dismantled, a preferable way to find the nature and existence of a flaw (crack or delamination) is in a nondestructive manner. Recently, it has been demonstrated by Spetzler and Datta (1990) that detection of flaws in plates can be carried out using guided ultrasonic waves. A schematic diagram of a typical ultrasonic flaw detector is shown in Figure 1.1. These flaw detection techniques use a transducer to send ultrasonic waves into the plate. The waves incident on the plate travel through the plate, and when a flaw is encountered they scatter into reflected and transmitted signals. Of particular interest is the scattered signal, which reaches the surface of the plate and is measured by receivers located on the suraface. The scattered signals carry a substantial amount of information about the size, shape, and location of the flaw. Ultrasonic flaw detection techniques rely on the theoretical predictions of amplitudes of scattered signals. The nature and the existence of the flaw is determined by comparing ultrasonic measurements of signal amplitudes with theoretical predictions. Therefore, it is crucial to have a theoretical model which can accurately predict the ultrasonic wave scattering by flaws.

The free edge of a plate can be considered as a through-thickness crack which is a special case of a normal edge crack. Thus reflection of waves at the free edge of a plate can be considered as a special case of a wave scattering problem. Torvik (1967) treated the free end reflection of a homogeneous isotropic plate by expanding the reflected wave field in wave functions. He determined the amplitudes of modes, approximately, by using a variational principle. Wu and Plunkett (1967) 
also addressed this problem by using a variational principle method and a residual boundary value minimization method. Gregory and Gladwell (1983) have reported a detailed investigation of symmetric Rayleigh-Lamb wave reflection at the edge of a homogeneous isotropic plate by using the method of projection. However, to our knowledge, a study of free end reflection of homogeneous anisotropic and laminated anisotropic plates has not been reported.

Many references on earlier work on problems of wave scattering by cracks can be found in the review article by Miklowitz (1960). The edited volume of Datta et al. (1990a) is a good source of reference on current works on wave scattering by cracks. Rokhlin $(1980,1981)$ has analysed the scattering of Lamb waves by a crack parallel to the surface of a homogeneous isotropic plate using the modified Wiener-Hopf technique and the method of multiple diffractions. Tan and Auld (1980) investigated the scattering of Lamb waves by a crack normal to the surface of a homogeneous isotropic plate using the normal mode variational method. Recently, Achenbach and his co-workers (Achenbach and Li, 1986; Achenbach and Kitahara, 1986; Angle and Achenbach, 1987; Mikata and Achenbach, 1988) studied the wave scattering by a periodic distribution of cracks and cavities in a homogeneous isotropic infinite solid. However, application of these methods is extremely difficult, if not impossible, for flaws of arbitrary shape.

Shah et al. (1982) and Wong (1985) used a hybrid method combining an analytical procedure with a finite element method to study the scattering of horizontally polarzed shear ( $\mathrm{SH}$ ) waves by arbitrarily shaped cracks and cavities located either at the surface or at a depth in a homogeneous isotropic half-space. Later, Abduljabbar et al. (1983) extended this hybrid method for the analysis of the scattering of SH waves by arbitrarily shaped flaws in homogeneous isotropic plates. Koshiba et al. (1984) extended this approach to Lamb wave scattering by flaws in homogeneous 
isotropic plates. Recently, Paskaramoorthy et al. (1989) used the hybrid method to investigate scattering of slow flexural waves by a crack in a homogeneous isotropic plate. Information on wave scattering by flaws in anisotropic plates is very limited. It is noted here that while this study was in progress, three other parallel investigations were underway. In one, Al-Nassar (1990) used the hybrid method to study Lamb wave scattering by weldments in isotropic plates. In the second, using the hybrid method, Bratton et al. (1990) studied scattering by a normal surface breaking crack in a homogeneous (uniaxial) fiber-reinforced composite plate. In the other, $\mathrm{Ju}$ et al. (1990) investigated scattering by a interior crack parallel to the surface of a homogeneous fiber-reinforced plate, using a different technique that combines finite elements and Green's function integral. However, wave scattering by cracks and delaminations in laminated composite plates has not been investigated.

\subsection{An Overview of the Present Study}

The objective of the present study is to investigate propagation and scattering of free guided waves in laminated composite plates with particular reference to material characterisation and flaw detection. The analysis is carried out on the assumption that the lateral dimensions of the plate are very large in comparison to the thickness of the plate. A stiffness method and an analytical method are used to study the dispersion characteristics of free guided waves. As a special case of a scattering problem, the free end reflection problem is investigated in detail using least-squares and variational methods. Wave scattering by cracks and delaminations is studied by extending the hybrid method.

\subsubsection{Guided Wave Propagation}

The stiffness method used in this study is an extension of that presented by 
Datta et al. (1988) to the off-axis case where direction of wave propagation is at an arbitrary angle to the fiber direction in each lamina. In this method, the dynamic behaviour of the composite plate is approximated by dividing the plate into several sublayers and representing the displacement distribution through the thickness of each sublayer by cubic interpolation polynomials. These polynomial functions involve a number of discrete generalized coordinates, which are displacements and tractions at the interfaces between the adjoining sublayers. By applying the Hamilton's principle, the dispersion equation is obtained as a standard algebraic eigenvalue problem. Eigenvalues and eigenvectors of this equation yield the frequencies of propagating, nonpropagating, and evanescent modes, and the associated displacements and tractions at the interfaces.

An efficient method to obtain the exact dispersion characteristics of a plate having arbitrary number of anisotropic layers has not been reported in the literature. Although it is possible to obtain, using a propagator matrix approach (see Mal, 1988), exact dispersion equation governing guided waves in a layered anisotropic plates, finding roots of this transcendental equation is quite cumbersome and computationally very expensive. As the number of layers increases, the exact dispersion relation becomes extremely complicated and requires robust search techniques in locating roots. To circumvent this difficulty, an analytical method that combines an efficient root locating scheme is proposed here. In this method, exact dispersion relation of the layered anisotropic plate is constructed using the propagator matrices. Mullers's method as given in Conte and Boor (1972) is then used, in conjunction with initial guesses obtained from an approximate theory, to obtain analytical dispersion characteristics.

The accuracy of the stiffness method is demonstrated by comparing the results with analytical results for homogeneous and layered fiber-reinforced plates. The ef- 
fect of anisotropy on the dispersion characteristics in a homogeneous fiber-reinforced plate is studied by changing the angle between the fiber direction and the direction of wave propagation. The effect of interface layers is investigated using an actual modeling approach. In this approach, the interface layer is considered as a sublayer, thus taking into account both the stiffness and the density of the bond layer. The results of the interface effects show the possibility of using ultrasonic techniques to characterise bond layer properties and thickness. Finally, the effect of number of layers on the dispersion characteristics in fiber-reinforced cross-ply laminated plates is investigated. It is shown that the material properties of a composite plate with a sufficiently large number of layers can be charaterised as homogeneous anisotropic with six elastic constants.

\subsubsection{Free End Reflection}

A wave function expansion procedure is used to solve the problem of reflection of waves normally incident upon the free edge of a semi-infinite, laminated composite plate. The reflected field consists of a finite number of propagating modes and an infinite number of nonpropagating and evanescent modes. A finite number of wave functions are superposed to represent the reflected field. The least-squares and variational principle methods are used to determine the unknown reflected wave amplitudes. The numerical results of wave amplitudes and division of energy among various reflected modes for a homogeneous fiber-reinforced plate and for a 35-layer cross-ply laminated plate are presented. The accuracy of the results are checked by satisfying the principle of energy conservation. The end resonance for the homogeneous fiber-reinforced composite plate is also reported. 


\subsubsection{Wave Scattering by Cracks and Delaminations}

In the present study, the hybrid method is extended to the analysis of wave scattering by flaws in laminated composite plates. The hybrid method combines finite element formulation in a bounded interior region of the plate with a wave function expansion representation in the exterior region. All the flaws are assumed to be contained in the bounded region. The two regions are connected along vertical boundaries. Continuity conditions for the displacement and interaction forces are imposed at the nodes lying on the boundaries. This results in a system of linear equations which is solved for the unknown wave function amplitudes. These amplitudes are used to obtain boundary nodal displacements and in turn to obtain interior nodal displacements. The hybrid method is illustrated for the case of scattering by a symmetric normal edge crack and a centrally located normal matrix crack that grows into delamination. Numerical results of the scattering problem are presented for an isotropic plate, a homogenous fiber-reinforced plate, and for 8-layer and 35-layer cross-ply laminated plates. The accuracy of the results are checked by the satisfaction of the reciprocity relations and the principle of energy conservation. The results of this investigation have potential applications in ultrasonic nondestructive evaluation.

\subsection{Organisation of the Thesis}

This thesis is concerned with the investigation of propagation and scattering of guided waves in laminated composite plates with particular reference to material characterisation and flaw detection. In Chaper 2, the formulation of an analytical and an approximate wave propagation model is presented. The accuracy of the approximate model and the numerical results for effects of interface bond layers, anisotropy, and layering on the dispersion characteristics are discussed in the same 
chapter. Formulation and discussion of numerical results of the free end reflection problem are presented in Chapter 3. In Chapter 4, the formulation of the hybrid method for wave scattering by flaws in laminated composite plates is given. Also given in the same chapter is the numerical results for scattering by a symmetric normal edge crack and a normal central crack that grows into delamination. Finally, conclusions of the research are presented in Chapter 5. Some recommendations for future work are also made in this chapter. 


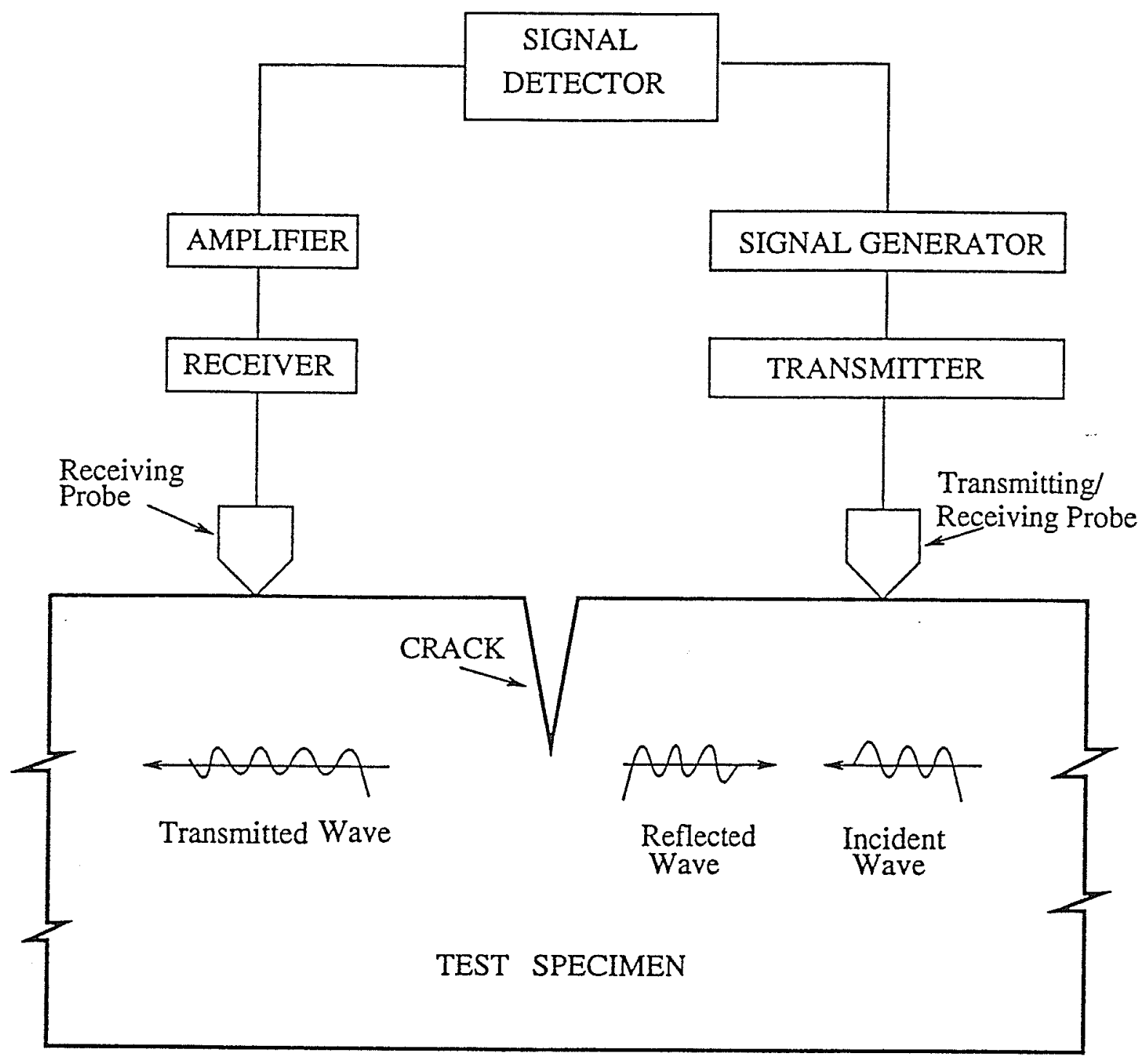

Figure 1.1 : Schematic diagram of a typical ultrasonic flaw detector. 


\section{C h a p t e 2}

\section{Wave Propagation in Laminated \\ Composite Plates}

\subsection{General}

In this chapter, wave propagation in laminated composite plates is investigated in detail. As mentioned in Chapter 1, two wave propagation models are developed: one employing an analytical formulation; and the other employing an approximate displacement and stress based stiffness method. The main objective of developing these models is to find the dispersion relations and thereby to study guided wave propagation in a laminated composite plate. The effects of interface layers, anisotropy, and layering are investigated by making use of dispersion characteristics obtained from these models. Later in this chapter, it is shown that the material properties of a composite plate with a large number of laminae can be characterised as homogeneous anisotropic.

\subsection{Description of the problem}

Time-harmonic elastic wave propagation in an infinite plate composed of perfectly bonded layers with possibly distinct mechanical properties and thickness is considered. The two faces of the plate $z=0$ and $z=H$ are traction-free, and the global rectangular cartesian coordinate system $(x, y, z)$ is as shown in Figure 2.1. The assumed direction of plane wave propagation is $x$. Since the plate is infinite in $y$ direction, all field quantities (dispalcements, strains, stresses etc.) are independent of the $y$ coordinate. 


\subsection{Analytical Method}

In this section, a wave propagation model based on exact analytical formulation is developed.

\subsubsection{Governing Equations}

Since the concern here is with a plate having a large and a varying number of layers, it will be convenient to resort to an analytical technique in which the number and properties of layers can be arbitrarily varied without substantially changing the solution procedure. In the technique adopted here, each layer is divided into several sublayers so that the total number of sublayers through the thickness, $H$, is $N$. For simplicity in the analysis, each layer is assumed to have tranversely isotropic material properties with the material symmetry axis making an arbitrary angle with the $x$ - axis. In general, waves propagating in a direction making an arbitrary angle with the symmetry axis of each lamina will produce a three dimensional wave motion. Let $t$ denotes time, and $u(x, z, t), v(x, z, t), w(x, z, t)$ denote particle displacement components in $x, y, z$ directions, respectively. Consider the $i$-th sublayer bounded by $z=z_{i}$ and $z=z_{i+1}$. A local rectangular cartesian coordinate system $(X, Y, Z)$ is chosen in the $i$-th sublayer with the origin in the mid-plane of the sublayer, $X$-axis along the material symmetry axis (fiber direction), $Z$ - axis vertically down, and $Y$ axis parallel to the plane of the plate. Let $U(X, Y, Z, t), V(X, Y, Z, t), W(X, Y, Z, t)$ be the displacement components of any particle within the $i$-th sublayer in $X, Y$, $Z$ directions, respectively.

The strain-displacement relations within the $i$-th sublayer in the local coordinate system are given by

$$
\epsilon_{X X}=\frac{\partial U}{\partial X}
$$




$$
\begin{aligned}
& \epsilon_{Y Y}=\frac{\partial V}{\partial Y} \\
& \epsilon_{Z Z}=\frac{\partial W}{\partial Z} \\
& \epsilon_{Y Z}=\frac{1}{2} \gamma_{Y Z}=\frac{1}{2}\left(\frac{\partial V}{\partial Z}+\frac{\partial W}{\partial Y}\right) \\
& \epsilon_{Z X}=\frac{1}{2} \gamma_{Z X}=\frac{1}{2}\left(\frac{\partial U}{\partial Z}+\frac{\partial W}{\partial X}\right) \\
& \epsilon_{X Y}=\frac{1}{2} \gamma_{X Y}=\frac{1}{2}\left(\frac{\partial V}{\partial X}+\frac{\partial U}{\partial Y}\right)
\end{aligned}
$$

where $\epsilon_{I J}$ represent strain components.

The stress-strain relation of this sublayer in the local coordinate system is given by (see Lekhnitskii, 1963)

$$
\left\{\begin{array}{c}
\sigma_{X X} \\
\sigma_{Y Y} \\
\sigma_{Z Z} \\
\sigma_{Y Z} \\
\sigma_{Z X} \\
\sigma_{X Y}
\end{array}\right\}=\left[\begin{array}{cccccc}
C_{11} & C_{12} & C_{13} & 0 & 0 & 0 \\
C_{12} & C_{22} & C_{23} & 0 & 0 & 0 \\
C_{13} & C_{23} & C_{33} & 0 & 0 & 0 \\
0 & 0 & 0 & C_{44} & 0 & 0 \\
0 & 0 & 0 & 0 & C_{55} & 0 \\
0 & 0 & 0 & 0 & 0 & C_{66}
\end{array}\right]\left\{\begin{array}{c}
\epsilon_{X X} \\
\epsilon_{Y Y} \\
\epsilon_{Z Z} \\
\gamma_{Y Z} \\
\gamma_{Z X} \\
\gamma_{X Y}
\end{array}\right\}
$$

where $\sigma_{I J}$ are the stress components and $C_{I J}$ are the elements of constitutive matrix for the sublayer. Note that

$$
C_{22}=C_{33}, \quad C_{55}=C_{66}, \quad C_{12}=C_{13}, \quad C_{44}=\frac{\left(C_{22}-C_{23}\right)}{2}
$$

Let $\theta$ be the angle between the global $x$ - axis and the local $X$-axis measured anticlockwise from the global $x$ - axis. The stress and strain components for the $i$-th sublayer in the global $(x, y, z)$ coordinate system are related by

$$
\left\{\begin{array}{c}
\sigma_{x x} \\
\sigma_{y y} \\
\sigma_{z z} \\
\sigma_{y z} \\
\sigma_{z x} \\
\sigma_{x y}
\end{array}\right\}=\left[\begin{array}{cccccc}
D_{11} & D_{12} & D_{13} & 0 & 0 & D_{16} \\
D_{12} & D_{22} & D_{23} & 0 & 0 & D_{26} \\
D_{13} & D_{23} & D_{33} & 0 & 0 & D_{36} \\
0 & 0 & 0 & D_{44} & D_{45} & 0 \\
0 & 0 & 0 & D_{45} & D_{55} & 0 \\
D_{16} & D_{26} & D_{36} & 0 & 0 & D_{66}
\end{array}\right]\left\{\begin{array}{c}
\epsilon_{x x} \\
\epsilon_{y y} \\
\epsilon_{z z} \\
\gamma_{y z} \\
\gamma_{z x} \\
\gamma_{x y}
\end{array}\right\}
$$


where $\sigma_{i j}$ and $\epsilon_{i j}$ represent stress and strain components, respectively, in the $(x, y, z)$ coordinate system and $D_{i j}$ are related to $C_{I J}$ by the equations presented in Appendix A.

In the absence of body forces, the equations of motion in the local coordinate system are given by

$$
\begin{aligned}
& \frac{\partial \sigma_{X X}}{\partial X}+\frac{\partial \sigma_{X Y}}{\partial Y}+\frac{\partial \sigma_{X Z}}{\partial Z}=\rho \frac{\partial^{2} U}{\partial t^{2}} \\
& \frac{\partial \sigma_{X Y}}{\partial X}+\frac{\partial \sigma_{Y Y}}{\partial Y}+\frac{\partial \sigma_{Y Z}}{\partial Z}=\rho \frac{\partial^{2} V}{\partial t^{2}} \\
& \frac{\partial \sigma_{X Z}}{\partial X}+\frac{\partial \sigma_{Y Z}}{\partial Y}+\frac{\partial \sigma_{Z Z}}{\partial Z}=\rho \frac{\partial^{2} W}{\partial t^{2}}
\end{aligned}
$$

where $\rho$ is the density of the sublayer under consideration.

If the direction of the fibers in each lamina is aligned with either $x$ - or $y$-axis, then the wave propagation problem reduces to two uncoupled problems: plane strain case in which the displacement components are $u$ and $w$, and antiplane strain case where the only nonzero displacement component is $v$. When the wave propagation occurs along an off-axis direction (i.e. $\theta \neq 0^{\circ}$ or $90^{\circ}$ ), the particle dispalcement vector will have all three displacement components: $u, v, w$. In $\S 2.3 .2$ analysis for plane strain case is presented. Since the analysis for antiplane strain case is very similar to the plane strain case, no details of the antiplane strain case are presented here. Off-axis case is dealt with in $\S 2.3 .3$.

\subsubsection{Plane Strain case}

The nonvanishing displacement components in this case are $u(x, z, t)$ and $w(x, z, t)$ in $x$ and $z$ directions, respectively. The governing equations (2.1), (2.4) and (2.5) for the $i$-th sublayer in the global $(x, y, z)$ coordinate system reduce, 
respectively, to:

$$
\begin{gathered}
\epsilon_{x x}=\frac{\partial u}{\partial x} \\
\epsilon_{z z}=\frac{\partial w}{\partial z} \\
\gamma_{z x}=\frac{\partial w}{\partial x}+\frac{\partial u}{\partial z} \\
\left\{\begin{array}{l}
\sigma_{x x} \\
\sigma_{z z} \\
\sigma_{z x}
\end{array}\right\}=\left[\begin{array}{ccc}
D_{11} & D_{13} & 0 \\
D_{13} & D_{33} & 0 \\
0 & 0 & D_{55}
\end{array}\right]\left\{\begin{array}{c}
\epsilon_{x x} \\
\epsilon_{z z} \\
\gamma_{z x}
\end{array}\right\},
\end{gathered}
$$

and

$$
\begin{aligned}
& \frac{\partial \sigma_{x x}}{\partial x}+\frac{\partial \sigma_{z x}}{\partial z}=\rho \frac{\partial^{2} u}{\partial t^{2}} \\
& \frac{\partial \sigma_{z x}}{\partial x}+\frac{\partial \sigma_{z z}}{\partial z}=\rho \frac{\partial^{2} w}{\partial t^{2}}
\end{aligned}
$$

Note that the analysis presented in this subsection is valid not only for transversely isotropic material properties but also for orthotropic material properties. In the latter case, equation (2.3) does not hold and the appropriate material property transformation equation from Appendix A has to be used.

The appropriate forms for $u$ and $w$, which satisfy equations (2.8), are

$$
\begin{aligned}
u & =j k\left(\Omega_{1}^{+}+B \Omega_{2}^{+}\right) \exp [j(k x-\omega t)], \\
w & =\left(A s_{1} \Omega_{1}^{-}+s_{2} \Omega_{2}^{-}\right) \exp [j(k x-\omega t)] .
\end{aligned}
$$

where

$$
\begin{aligned}
& \Omega_{1}^{+}=a_{11} \cos \left(s_{1} z\right)+a_{12} \sin \left(s_{1} z\right), \\
& \Omega_{1}^{-}=a_{12} \cos \left(s_{1} z\right)-a_{11} \sin \left(s_{1} z\right), \\
& \Omega_{2}^{+}=a_{21} \cos \left(s_{2} z\right)+a_{22} \sin \left(s_{2} z\right), \\
& \Omega_{2}^{-}=a_{22} \cos \left(s_{2} z\right)-a_{21} \sin \left(s_{2} z\right),
\end{aligned}
$$




$$
\begin{aligned}
& A=\frac{k_{1}^{2}-\lambda_{1} k^{2}-s_{1}^{2}}{\delta_{1} s_{1}^{2}}, \\
& B=\frac{k_{1}^{2}-k^{2}-\beta_{1} s_{2}^{2}}{\delta_{1} k^{2}}
\end{aligned}
$$

$$
\begin{aligned}
{s_{1}}^{2}, s_{2}{ }^{2} & =\frac{k_{1}{ }^{2}\left(1+\beta_{1}\right)-k^{2} \eta_{1} \pm \Gamma_{1}}{2 \beta_{1}} \\
\Gamma_{1} & =\sqrt{\left[k^{2} \eta_{1}-k_{1}{ }^{2}\left(1+\beta_{1}\right)\right]^{2}-4 \beta_{1}\left(\lambda_{1} k^{2}-k_{1}{ }^{2}\right)\left(k^{2}-k_{1}{ }^{2}\right)}
\end{aligned}
$$

$$
\begin{array}{r}
\lambda_{1}=\frac{D_{11}}{D_{55}}, \quad \beta_{1}=\frac{D_{33}}{D_{55}}, \quad \delta_{1}=1+\frac{D_{13}}{D_{55}} \\
\eta_{1}=1+\lambda_{1} \beta_{1}-\delta_{1}{ }^{2}, \quad k_{1}=\sqrt{\frac{\rho \omega^{2}}{D_{55}}}
\end{array}
$$

In the above, $k$ is the wavenumber in $x$ direction; $\omega$ is the circular frequency; $j=\sqrt{-1} ;$ and $a_{11}, a_{12}, a_{21}$, and $a_{22}$ are arbitrary constants for the sublayer. Stress and displacement components at any point within the sublayer can be expressed in terms of the four unknown constants $a_{11}, a_{12}, a_{21}$, and $a_{22}$. By evaluating the stresses and displacements at $z=z_{i}$ and $z=z_{i+1}$, and by eliminating the four unknown constants, the following relation can be obtained:

$$
\left\{B_{i+1}\right\}=\left[P_{i}\right]\left\{B_{i}\right\}
$$

where

$$
\left\{B_{i}\right\}^{\mathrm{T}}=\left\langle u_{i} \quad w_{i} \quad \sigma_{z z i} \quad \sigma_{z x i}\right\rangle
$$

The vector quantity $\left\{B_{i}\right\}$, which is unknown yet, represents the displacement and stress components at $z=z_{i} ;\left\{B_{i+1}\right\}$ represents the same quantities at $z=z_{i+i}$; 
superscript $\mathrm{T}$ denotes the matrix transpose; and $\left[P_{i}\right]$ is the propagator matrix for the $i$-th sublayer. The elements of $\left[P_{i}\right]$ are given in Appendix B.

The global propagator matrix $[P]$ for the entire plate is obtained by the repeated application of equation (2.14) as

$$
\left\{B_{N+1}\right\}=[P]\left\{B_{1}\right\},
$$

where

$$
[P]=\left[P_{N}\right]\left[P_{N-1}\right] \cdots\left[P_{i}\right] \cdots\left[P_{2}\right]\left[P_{1}\right]
$$

The repeated application of equation (2.14) ensures the continuity of the displacement components $u$ and $w$, and stress components $\sigma_{z z}$ and the $\sigma_{z x}$, at the interfaces between sublayers. Denoting the elements of the 4 by 4 matrix $[P]$ by $P_{m n}$ $(m, n=1,2,3,4)$ and invoking the zero traction conditions at interfaces 1 and $(N+1)$, the following can be obtained from equation (2.16):

$$
\left[\begin{array}{ll}
P_{31} & P_{32} \\
P_{41} & P_{42}
\end{array}\right]\left\{\begin{array}{l}
u_{1} \\
w_{1}
\end{array}\right\}=\left\{\begin{array}{l}
0 \\
0
\end{array}\right\} \text {. }
$$

The exact dispersion equation (frequency equation) for the plate is obtained by setting the determinant of the coefficient matrix to zero as

$$
f(\omega, k)=\left|\begin{array}{ll}
P_{31} & P_{32} \\
P_{41} & P_{42}
\end{array}\right|=0 .
$$

Equation (2.19) can be solved for $k$, for given $\omega$, or alternatively, it can be solved for $\omega$, for given $k$. The details of the method employed to determine the roots of the dispersion equation will be discussed in $\S 2.5$.

Once the roots of the dispersion equation are determined, the corresponding wave functions (displacement and stress eigenvectors) can be obtained using equation (2.18) and the successive application of equation (2.14) at interfaces. It should 
be noted at this stage that division of layers into sublayers is not required to obtain the exact dispersion equation but is required to calculate the values of wave funtions at a sufficiently large number of points through the thickness. The usefulness of the subdivision will be evident in Chapters 3 and 4 .

If the problem under consideration is symmetric or antisymmetric, it is possible to model only the half-thickness of the plate in the analysis. In this case, the boundary conditions at the middle surface of the plate, $z=H / 2$, are :

$$
\begin{aligned}
& w=0 \quad ; \quad \sigma_{z x}=0, \quad \text { for symmetric problems, } \\
& u=0 ; \quad \sigma_{z z}=0, \quad \text { for antisymmetric problems. }
\end{aligned}
$$

Application of these boundary conditions in equation (2.16) results in the following dispersion equations:

$$
\left|\begin{array}{ll}
P_{21} & P_{22} \\
P_{41} & P_{42}
\end{array}\right|=0, \quad \text { for symmetric problems, }
$$

and

$$
\left|\begin{array}{ll}
P_{11} & P_{12} \\
P_{31} & P_{32}
\end{array}\right|=0, \quad \text { for antisymmetric problems. }
$$

\subsubsection{Off-axis Case}

In order to solve the governing equations for this case, it will be neccessary to manipulate them in such a way that tractable differential equations are obtained. For this purpose, it is convenient to carry out the analysis for the $i$-th sublayer in the local coordinate system first and use appropriate transformations to global coordinate system later on. In order to uncouple the governing equations, three potential functions $\Theta, \Phi$, and $\Psi$ are introduced, as defined below:

$$
U=\frac{\partial \Theta}{\partial X},
$$




$$
\begin{aligned}
& V=\frac{\partial \Phi}{\partial Y}+\frac{\partial \Psi}{\partial Z} \\
& W=\frac{\partial \Phi}{\partial Z}-\frac{\partial \Psi}{\partial Y}
\end{aligned}
$$

In view of equations (2.1), (2.2), and (2.23), the stress components can be written as

$$
\begin{aligned}
& \sigma_{X X}=C_{11} \frac{\partial^{2} \Theta}{\partial X^{2}}+C_{13}\left(\frac{\partial^{2} \Phi}{\partial Y^{2}}+\frac{\partial^{2} \Phi}{\partial Z^{2}}\right) \\
& \sigma_{Y Y}=C_{13} \frac{\partial^{2} \Theta}{\partial X^{2}}+C_{33}\left(\frac{\partial^{2} \Phi}{\partial Y^{2}}+\frac{\partial^{2} \Psi}{\partial Y \partial Z}\right)+C_{23}\left(\frac{\partial^{2} \Phi}{\partial Z^{2}}-\frac{\partial^{2} \Psi}{\partial Z \partial Y}\right) \\
& \sigma_{Z Z}=C_{13} \frac{\partial^{2} \Theta}{\partial X^{2}}+C_{23}\left(\frac{\partial^{2} \Phi}{\partial Y^{2}}+\frac{\partial^{2} \Psi}{\partial Y \partial Z}\right)+C_{33}\left(\frac{\partial^{2} \Phi}{\partial Z^{2}}-\frac{\partial^{2} \Psi}{\partial Z \partial Y}\right) \\
& \sigma_{Y Z}=C_{44}\left(2 \frac{\partial^{2} \Phi}{\partial Y \partial Z}+\frac{\partial^{2} \Psi}{\partial Z^{2}}-\frac{\partial^{2} \Psi}{\partial Y^{2}}\right) \\
& \sigma_{Z X}=C_{55}\left(\frac{\partial^{2} \Theta}{\partial Z \partial X}+\frac{\partial^{2} \Phi}{\partial X \partial Z}-\frac{\partial^{2} \Psi}{\partial X \partial Y}\right) \\
& \sigma_{X Y}=C_{55}\left(\frac{\partial^{2} \Phi}{\partial X \partial Y}+\frac{\partial^{2} \Psi}{\partial X \partial Z}+\frac{\partial^{2} \Theta}{\partial Y \partial X}\right)
\end{aligned}
$$

Substitution of equations (2.23) and (2.24) in equations (2.5), and subsequent algebraic manipulation of the resulting equations leads to the following three equations:

$$
\begin{gathered}
C_{55} \frac{\partial^{2}}{\partial X^{2}}\left(\nabla^{2} \Theta\right)+\left(C_{13}+C_{55}\right) \frac{\partial^{2}}{\partial X^{2}}\left(\nabla^{2} \Phi\right)+\left(C_{11} \frac{\partial^{2}}{\partial X^{2}}-\rho \frac{\partial^{2}}{\partial t^{2}}\right) \frac{\partial^{2} \Theta}{\partial X^{2}}=0 \\
\left(C_{13}+C_{55}\right) \frac{\partial^{2}}{\partial X^{2}}\left(\nabla^{2} \Theta\right)+C_{55} \frac{\partial^{2}}{\partial X^{2}}\left(\nabla^{2} \Phi\right)+C_{33} \nabla^{4} \Phi-\rho \frac{\partial^{2}}{\partial t^{2}}\left(\nabla^{2} \Phi\right)=0 \\
C_{44} \nabla^{4} \Psi+\left(C_{55} \frac{\partial^{2}}{\partial X^{2}}-\rho \frac{\partial^{2}}{\partial t^{2}}\right) \nabla^{2} \Psi=0
\end{gathered}
$$

where

$$
\begin{aligned}
& \nabla^{2}=\frac{\partial^{2}}{\partial Y^{2}}+\frac{\partial^{2}}{\partial Z^{2}} \\
& \nabla^{4}=\frac{\partial^{4}}{\partial Y^{4}}+2 \frac{\partial^{4}}{\partial Y^{2} \partial Z^{2}}+\frac{\partial^{4}}{\partial Z^{4}} .
\end{aligned}
$$


Equation (2.25a) is obtained by partial differentiation of equation (2.5a) writh respect to $X$. Equation (2.25b) is obtained by summation of the partial derivative of equation (2.5b) with respect to $Y$, and the partial derivative of equation (2.5c) with respect to $Z$. The partial derivative of equation (2.5c) with respect to $Y$ substracted from the partial derivative of equation (2.5b) with respect to $Z$, yields equation $(2.25 \mathrm{c})$.

It can be seen that equations (2.25a) and (2.25b) are coupled while equation $(2.25 \mathrm{c})$ is independent. The basic unknowns of the problem posed now are $\Theta, \Phi$, and $\Psi$. Since time-harmonic waves propagating in $x$ direction are considered, the $x$ and $t$ variation of unknown functions can be assumed to be of the form $\exp [j(k x-\omega t)]$ (where $k$ denotes the wavenumber in $x$ direction). On the other hand, since the plate is infinite in $y$ direction, unknown functions should not have any dependence on $y$. Therefore, the appropriate forms for the potential funtions are

$$
\begin{aligned}
& \Theta=f_{1}(Z) \exp (j \psi), \\
& \Phi=f_{2}(Z) \exp (j \psi) \\
& \Psi=f_{3}(Z) \exp (j \psi)
\end{aligned}
$$

where

$$
\begin{aligned}
\psi & =k x-\omega t=K X+L Y-\omega t \\
K & =k \cos \theta \\
L & =k \sin \theta
\end{aligned}
$$

In the above, $f_{1}(Z), f_{2}(Z)$, and $f_{3}(Z)$ are unknown functions; and $K$ and $L$ represent wavenumbers in $X$ and $Y$ directions, respectively, for the $i$-th sublayer. Substitution of equations (2.26) in equations (2.25) results in

$$
\left(\frac{d^{2}}{d Z^{2}}-L^{2}-\lambda_{2} K^{2}+k_{2}^{2}\right) f_{1}+\delta_{2}\left(\frac{d^{2}}{d Z^{2}}-L^{2}\right) f_{2}=0
$$




$$
\begin{gathered}
-\delta_{2} K^{2}\left(\frac{d^{2}}{d Z^{2}}-L^{2}\right) f_{1}+\left[\left(k_{2}{ }^{2}-K^{2}\right)+\beta_{2}\left(\frac{d^{2}}{d Z^{2}}-L^{2}\right)\right]\left(\frac{d^{2}}{d Z^{2}}-L^{2}\right) f_{2}=0 \\
{\left[-K^{2}+k_{2}{ }^{2}+\varepsilon_{2}\left(\frac{d^{2}}{d Z^{2}}-L^{2}\right)\right]\left(\frac{d^{2}}{d Z^{2}}-L^{2}\right) f_{3}=0}
\end{gathered}
$$

where

$$
\begin{aligned}
\lambda_{2}=\frac{C_{11}}{C_{55}}, \quad \beta_{2}=\frac{C_{33}}{C_{55}}, & \varepsilon_{2}=\frac{C_{44}}{C_{55}} \\
\delta_{2}=1+\frac{C_{13}}{C_{55}}, & k_{2}=\sqrt{\frac{\rho \omega^{2}}{C_{55}}} .
\end{aligned}
$$

The solution which satisfies equations (2.28) can be written as

$$
\begin{aligned}
& f_{1}=\Omega_{1}^{+(o)}+B^{(o)} \Omega_{2}^{+(o)}, \\
& f_{2}=A^{(o)} \Omega_{1}^{+(o)}+\Omega_{2}^{+(o)}, \\
& f_{3}=\Omega_{3}^{-(o)}
\end{aligned}
$$

where

$$
\begin{gathered}
\Omega_{1}^{+(o)}=a_{11}^{(o)} \cos \left(r_{1} Z\right)+a_{12}^{(o)} \sin \left(r_{1} Z\right), \\
\Omega_{2}^{+(o)}=a_{21}^{(o)} \cos \left(r_{2} Z\right)+a_{22}^{(o)} \sin \left(r_{2} Z\right), \\
\Omega_{3}^{-(o)}=a_{32}^{(o)} \cos (\zeta Z)-a_{31}^{(o)} \sin (\zeta Z), \\
A^{(o)}=\frac{{k_{2}}^{2}-\lambda_{2} K^{2}-\left(r_{1}{ }^{2}+L^{2}\right)}{\delta_{2}\left(r_{1}{ }^{2}+L^{2}\right)}, \\
B^{(o)}=\frac{{k_{2}}^{2}-K^{2}-\beta_{2}\left(r_{2}{ }^{2}+L^{2}\right)}{\delta_{2} K^{2}} \\
r_{1}{ }^{2}, r_{2}{ }^{2}=\frac{k_{2}{ }^{2}\left(1+\beta_{2}\right)-K^{2} \eta_{2} \pm \Gamma_{2}}{2 \beta_{2}} \\
\Gamma_{2}=\sqrt{\left[K^{2} \eta_{2}-k_{2}{ }^{2}\left(1+\beta_{2}\right)\right]^{2}-4 \beta_{2}\left(\lambda_{2} K^{2}-k_{2}{ }^{2}\right)\left(K^{2}-k_{2}{ }^{2}\right)},
\end{gathered}
$$




$$
\begin{gathered}
\zeta^{2}=\frac{k_{2}{ }^{2}-K^{2}-\varepsilon_{2} L^{2}}{\varepsilon_{2}} \\
\eta_{2}=1+\lambda_{2} \beta_{2}-\delta_{2}{ }^{2}
\end{gathered}
$$

In equations $(2.31), a_{11}^{(o)}, a_{12}^{(o)}, a_{21}^{(o)}, a_{22}^{(o)}, a_{31}^{(o)}$, and $a_{32}^{(o)}$ are arbitrary constants for the sublayer. The superscript $(o)$, used above and in the subsequent analysis, represents the off-axis case.

After dropping the factor $\exp (j \psi)$, the displacement and stress components in local coordinate system can now be written as

$$
\begin{gathered}
U=j K\left(\Omega_{1}^{+(o)}+B^{(o)} \Omega_{2}^{+(o)}\right) \\
V=j L\left(\Omega_{2}^{+(o)}+A^{(o)} \Omega_{1}^{+(o)}\right)-\zeta \Omega_{3}^{+(o)} \\
W=\left(r_{2} \Omega_{2}^{-(o)}+A^{(o)} r_{1} \Omega_{1}^{-(o)}\right)-j L \Omega_{3}^{-(o)} \\
\sigma_{X X}=C_{55}\left\{\left[-\lambda_{2} K^{2}+A^{(o)}\left(1-\delta_{2}\right)\left(r_{1}^{2}+L^{2}\right)\right] \Omega_{1}^{+(o)}\right. \\
\left.+\left[-\lambda_{2} K^{2} B^{(o)}+\left(1-\delta_{2}\right)\left(r_{2}{ }^{2}+L^{2}\right)\right] \Omega_{2}^{+(o)}\right\} \\
\sigma_{Y Y}=C_{55}\left\{\left[\left(1-\delta_{2}\right) K^{2}+A^{(o)}\left(2 \varepsilon_{2} r_{1}{ }^{2}-\beta_{2}\left(r_{1}{ }^{2}+L^{2}\right)\right)\right] \Omega_{1}^{+(o)}\right. \\
\left.+\left[\left(1-\delta_{2}\right) K^{2} B^{(o)}+2 \varepsilon_{2} r_{2}{ }^{2}-\beta_{2}\left(r_{2}{ }^{2}+L^{2}\right)\right] \Omega_{2}^{+(o)}-2 \varepsilon_{2} j L \zeta \Omega_{3}^{+(o)}\right\}, \\
\sigma_{Z Z}=C_{55}\left\{\left[\left(1-\delta_{2}\right) K^{2}+A^{(o)}\left(2 \varepsilon_{2} L^{2}-\beta_{2}\left(r_{1}{ }^{2}+L^{2}\right)\right)\right] \Omega_{1}^{+(o)}\right. \\
\left.+\left[\left(1-\delta_{2}\right) K^{2} B^{(o)}+2 \varepsilon_{2} L^{2}-\beta_{2}\left(r_{2}{ }^{2}+L^{2}\right)\right] \Omega_{2}^{+(o)}+2 \varepsilon_{2} j L \zeta \Omega_{3}^{+(o)}\right\},(2) \\
\sigma_{Y Z}=C_{55}\left\{2 j L r_{1} A^{(o)} \Omega_{1}^{-(o)}+2 j L r_{2} \Omega_{2}^{-(o)}+\left(L^{2}-\zeta^{2}\right) \Omega_{3}^{-(o)}\right\} \\
\sigma_{Z X}=C_{55} K\left\{j r_{1}\left(1+A^{(o)}\right) \Omega_{1}^{-(o)}+j r_{2}\left(1+B^{(o)}\right) \Omega_{2}^{-(o)}+L \Omega_{3}^{-(o)}\right\} \\
\sigma_{X Y}=-C_{55} K\left\{L\left(1+A^{(o)}\right) \Omega_{1}^{+(o)}+L\left(1+B^{(o)}\right) \Omega_{2}^{+(o)}+j \zeta \Omega_{3}^{+(o)}\right\}
\end{gathered}
$$


where

$$
\begin{aligned}
& \Omega_{1}^{-(o)}=a_{12}^{(o)} \cos \left(r_{1} Z\right)-a_{11}^{(o)} \sin \left(r_{1} Z\right), \\
& \Omega_{2}^{-(o)}=a_{22}^{(o)} \cos \left(r_{2} Z\right)-a_{21}^{(o)} \sin \left(r_{2} Z\right), \\
& \Omega_{3}^{+(o)}=a_{31}^{(o)} \cos (\zeta Z)+a_{32}^{(o)} \sin (\zeta Z),
\end{aligned}
$$

The next step in the analysis is to transform the displacements and stresses from the local coordinate system to the global coordinate system. These transformations are defined by

$$
\begin{aligned}
& u=m U+n V, \\
& v=-n U+m V, \\
& w=W
\end{aligned}
$$

and

$$
\begin{aligned}
& \sigma_{x x}=m^{2} \sigma_{X X}+n^{2} \sigma_{Y Y}+2 m n \sigma_{X Y}, \\
& \sigma_{y y}=n^{2} \sigma_{X X}+m^{2} \sigma_{Y Y}-2 m n \sigma_{X Y}, \\
& \sigma_{x y}=m n\left(\sigma_{Y Y}-\sigma_{X X}\right)+\left(m^{2}-n^{2}\right) \sigma_{X Y}, \\
& \sigma_{z x}=m \sigma_{Z X}+n \sigma_{Y Z}, \\
& \sigma_{y z}=-n \sigma_{Z X}+m \sigma_{Y Z}, \\
& \sigma_{z z}=\sigma_{Z Z},
\end{aligned}
$$

respectively, where $m=\sin \theta$ and $n=\cos \theta$.

The six arbitrary constants $a_{11}^{(o)}, a_{12}^{(o)}, a_{21}^{(o)}, a_{22}^{(o)}, a_{31}^{(o)}, a_{32}^{(o)}$ can be eliminated by evaluating the displacement components $u, v, w$, and the stress components $\sigma_{z z}$, 
$\sigma_{z x}, \sigma_{z y}$ at $z=z_{i}$ and $z=z_{i+1}$. This leads to

$$
\left\{B_{i+1}^{(o)}\right\}=\left[P_{i}^{(o)}\right]\left\{B_{i}^{(o)}\right\}
$$

where

$$
\left\{B_{i}^{(o)}\right\}^{\mathrm{T}}=\left\langle\begin{array}{llllll}
u_{i} & v_{i} & \sigma_{z z i} & \sigma_{z x i} & \sigma_{y z i} & w_{i}
\end{array}\right\rangle
$$

and $\left[P_{i}^{(o)}\right]$ is the propagator matrix for the $i$-th sublayer, which is defined in Appendix B.

The global propagator matrix $\left[P^{(o)}\right]$ for the entire plate is obtained by the repeated application of equation (2.41) as

$$
\left\{B_{N+1}^{(o)}\right\}=\left[P^{(o)}\right]\left\{B_{1}^{(o)}\right\}
$$

where

$$
\left[P^{(o)}\right]=\left[P_{N}^{(o)}\right]\left[P_{N-1}^{(o)}\right] \cdots\left[P_{i}^{(o)}\right] \cdots\left[P_{2}^{(o)}\right]\left[P_{1}^{(o)}\right]
$$

The repeated application of equation (2.41) ensures the continuity of the displacement components $u, v, w$, and the stress components $\sigma_{z z}, \sigma_{z x}, \sigma_{z y}$ at the interfaces between sublayers. Let the elements of the 6 by 6 matrix $\left[P^{(o)}\right]$ be denoted by $P_{m n}^{(o)}$ $(m, n=1,2, \cdots, 6)$. Invoking the zero traction conditions at the interfaces 1 and $(N+1)$, the following is obtained from equation (2.43):

$$
\left[\begin{array}{lll}
P_{31}^{(o)} & P_{32}^{(o)} & P_{36}^{(o)} \\
P_{41}^{(o)} & P_{42}^{(o)} & P_{46}^{(o)} \\
P_{51}^{(o)} & P_{52}^{(o)} & P_{56}^{(o)}
\end{array}\right]\left\{\begin{array}{c}
u_{1} \\
v_{1} \\
w_{1}
\end{array}\right\}=\left\{\begin{array}{l}
0 \\
0 \\
0
\end{array}\right\}
$$

The exact dispersion equation (frequency equation) for the plate is obtained by setting the determinant of the coefficient matrix in equation (2.45) to zero as

$$
f^{(o)}(\omega, k)=\left|\begin{array}{lll}
P_{31}^{(o)} & P_{32}^{(o)} & P_{36}^{(o)} \\
P_{41}^{(o)} & P_{42}^{(o)} & P_{46}^{(o)} \\
P_{51}^{(o)} & P_{52}^{(o)} & P_{56}^{(o)}
\end{array}\right|=0 .
$$


As in the plane strain case, if the problem under consideration is symmetric or antisymmetric, only a half-thickness of the plate is modeled in the analysis. In this case, the boundary conditions at the middle surface of the plate, $z=H / 2$, are :

$$
\begin{array}{llll}
w=0 ; & \sigma_{z x}=0 ; & \sigma_{y z}=0, & \text { for symmetric problems } \\
u=0 ; & ; \quad v=0 \quad ; \quad \sigma_{z z}=0, & \text { for antisymmetric problems. }
\end{array}
$$

Applying these boundary conditions in equation (2.43), the dispersion equations are obtained as

$$
\left|\begin{array}{lll}
P_{41}^{(o)} & P_{42}^{(o)} & P_{46}^{(o)} \\
P_{51}^{(o)} & P_{52}^{(o)} & P_{56}^{(o)} \\
P_{61}^{(o)} & P_{62}^{(o)} & P_{66}^{(o)}
\end{array}\right|=0, \quad \text { for symmetric problems }
$$

and

$$
\left|\begin{array}{lll}
P_{11}^{(o)} & P_{12}^{(o)} & P_{16}^{(o)} \\
P_{21}^{(o)} & P_{22}^{(o)} & P_{26}^{(o)} \\
P_{31}^{(o)} & P_{32}^{(o)} & P_{36}^{(o)}
\end{array}\right|=0, \quad \text { for antisymmetric problems. }
$$

\subsection{Stiffness Method}

Dong and his co-workers (Dong and Nelson, 1972; Dong and Pauley, 1978; Dong and Huang, 1985) presented a numerical technique applicable for wave propagation analysis in a layered anisotropic plate. In their technique, thickness variations of the displacements are approximated by quadratic functions of a thickness variable. The generalized coordinates in this representation are the displacements at the top, middle, and bottom of each mathematical layer (sublayer). Datta et al.(1988) presented a stiffness method that employs a higher order polynomial representation where generalized coordinates are displacements and tractions at the top and bottom of each mathematical layer. In their analysis, only plane strain and antiplane 
strain problems were considered. Herein, this stiffness method is extended to incorperate off-axis propagation where direction of wave propagation is at an aribitrary angle to the fiber direction in each lamina. For convenience, in what follows in this thesis, the present stiffness method is referred to as stiffness method I whilst the method of Dong and his co-workers is referred to as stiffness method II. As in the case of the analytical method presented in $\$ 2.3$, the stiffness method I starts with dividing each layer into several sublayers so that the total number of sublayers through the thickness of the plate is $N$. For transversely isotropic material properties, the stress-strain relation within the $i$-th sublayer is given by equation (2.2) and equation (2.4) in the local and the global coordinate systems, respectively. By using the interpolation polynomials in the $z$ direction, the displacement components can be approximated as

$$
\begin{aligned}
& u=u_{i} n_{1}+u_{i+1} n_{2}+u_{i}^{*} n_{3}+u_{i+1}^{*} n_{4}, \\
& v=v_{i} n_{1}+v_{i+1} n_{2}+v_{i}^{*} n_{3}+v_{i+1}^{*} n_{4}, \\
& w=w_{i} n_{1}+w_{i+1} n_{2}+w_{i}^{*} n_{3}+w_{i+1}^{*} n_{4},
\end{aligned}
$$

where $n_{1}, n_{2}, n_{3}$, and $n_{4}$ are cubic polynomials in the local coordinate $Z$ given by

$$
\begin{aligned}
n_{1} & =\frac{1}{4}\left(2-3 \eta+\eta^{3}\right), \\
n_{2} & =\frac{1}{4}\left(2+3 \eta-\eta^{3}\right), \\
n_{3} & =\frac{h}{4}\left(1-\eta-\eta^{2}+\eta^{3}\right), \\
n_{4} & =\frac{h}{4}\left(-1-\eta+\eta^{2}+\eta^{3}\right),
\end{aligned}
$$

and

$$
\eta=\frac{Z}{h}
$$


In the above, $u_{i}, v_{i}$, and $w_{i}$ are the values of $u, v$, and $w$, respectively, at the $i$-th interface. Subscript $i+1$ denotes the same quantities at the $(i+1)$-th interface. In addition, $u_{i}^{*}, v_{i}^{*}, w_{i}^{*}$, and $u_{i+1}^{*}, v_{i+1}^{*}, w_{i+1}^{*}$ represent some unknown coefficients associated with $i$-th and $(i+1)$-th interfaces, respectively. Using strain-displacement relations and stress-strain relations along with equation $(2.50)$, stress components at the sublayer interfaces can be expressed in terms of displacement components and unknown coefficients associated with the interfaces of the sublayer. This allows one to determine unknown coefficients as

$$
\begin{aligned}
u_{i}^{*} & =\frac{D_{44}}{\Delta} \chi_{i}-\frac{D_{45}}{\Delta} \tau_{i}-\frac{\partial w_{i}}{\partial x} \\
v_{i}^{*} & =\frac{D_{55}}{\Delta} \tau_{i}-\frac{D_{45}}{\Delta} \chi_{i} \\
w_{i}^{*} & =\frac{\sigma_{i}}{D_{33}}-\frac{D_{13}}{D_{33}} \frac{\partial u_{i}}{\partial x}-\frac{D_{36}}{D_{33}} \frac{\partial v_{i}}{\partial x}
\end{aligned}
$$

where

$$
\Delta=D_{44} D_{55}-D_{45}^{2}
$$

and $\chi_{i}, \tau_{i}, \sigma_{i}$ are the values of $\sigma_{z x}, \sigma_{y z}, \sigma_{z z}$, respectively, at the $i$-th interface. The corresponding expressions for $u_{i+1}^{*}, v_{i+1}^{*}$, and $w_{i+1}^{*}$ are obtained from equations (2.53) by simply replacing $i$ with $i+1$.

The equations governing the nodal generalized coordinates $u_{i}, v_{i}, w_{i}, \chi_{i}, \tau_{i}$, and $\sigma_{i} \quad(i=1,2, \cdots, N+1)$ are obtained using the Hamilton's principle. For this purpose, the Lagrangian $L_{i}$ per unit length in the $y$ direction for the $i$-th sublayer is written as

$$
L_{i}=\frac{1}{2} \int\left[\int_{-h}^{h}\left(\rho\{\dot{\bar{u}}\}^{\mathrm{T}}\{\dot{u}\}-\{\bar{\epsilon}\}^{\mathrm{T}}[D]\{\epsilon\}\right) d z\right] d x
$$

where

$$
\begin{aligned}
& \{u\}^{\mathrm{T}}=\left\langle\begin{array}{llllll}
u & v & w
\end{array}\right) \\
& \{\epsilon\}^{\mathrm{T}}=\left(\begin{array}{llllll}
\epsilon_{x x} & \epsilon_{y y} & \epsilon_{z z} & \gamma_{y z} & \gamma_{z x} & \gamma_{x y}
\end{array}\right\rangle,
\end{aligned}
$$


and $[D]$ is the constitutive matrix, the elements of which are the $D_{i j}$ in equation (2.4). The overbar and overdot denote, respectively, the complex conjugate and the derivative with respect to time.

Using equation (2.50) in strain-displacement relations, and in turn in equation $(2.55), L_{i}$ can be written as

$$
\begin{aligned}
L_{i}=\frac{1}{2} \int[ & {\left[\dot{q}^{\prime}\right\}^{\mathrm{T}}\left[c_{2}\right]\left\{\dot{q}^{\prime}\right\}+\left\{\dot{\bar{q}}^{\prime}\right\}^{\mathrm{T}}\left[c_{3}\right]\{\dot{q}\}+\{\dot{\bar{q}}\}^{\mathrm{T}}\left[c_{3}\right]\left\{\dot{q}^{\prime}\right\}+\{\dot{\bar{q}}\}^{\mathrm{T}}[m]\{\dot{q}\}-\left\{\dot{\bar{q}}^{\prime \prime}\right\}^{\mathrm{T}}\left[e_{1}\right]\left\{q^{\prime \prime}\right\} } \\
& -\left\{\bar{q}^{\prime \prime}\right\}^{\mathrm{T}}\left[e_{2}\right]\left\{q^{\prime}\right\}-\left\{\bar{q}^{\prime \prime}\right\}^{\mathrm{T}}\left[e_{3}\right]\{q\}-\left\{\bar{q}^{\prime}\right\}^{\mathrm{T}}\left[e_{2}\right]^{\mathrm{T}}\left\{q^{\prime \prime}\right\}-\left\{\bar{q}^{\prime}\right\}^{\mathrm{T}}\left[e_{4}\right]\left\{q^{\prime}\right\} \\
& \left.-\left\{\bar{q}^{\prime}\right\}^{\mathrm{T}}\left[e_{5}\right]\{q\}-\{\bar{q}\}^{\mathrm{T}}\left[e_{3}\right]^{\mathrm{T}}\left\{q^{\prime \prime}\right\}-\{\bar{q}\}^{\mathrm{T}}\left[e_{5}\right]^{\mathrm{T}}\left\{q^{\prime}\right\}-\{\bar{q}\}^{\mathrm{T}}\left[e_{6}\right]\{q\}\right] d x(2.56)
\end{aligned}
$$

where $\{q\}$ is defined as

$$
\{q\}^{\mathrm{T}}=\left\langle\begin{array}{llllllllllll}
u_{i} & \chi_{i} & v_{i} & \tau_{i} & w_{i} & \sigma_{i} & u_{i+1} & \chi_{i+1} & v_{i+1} & \tau_{i+1} & w_{i+1} & \sigma_{i+1}
\end{array}\right\rangle,
$$

and the primes denote derivatives with respect to $x$. The matrices $\left[c_{2}\right],\left[c_{3}\right],[m]$ and $\left[e_{1}\right]$ through $\left[e_{6}\right]$ are defined in Appendix C.

The Lagrangian for the entire plate is obtained by summation over all the sublayers, and its first variation leads to an approximate governing equation for the plate. Assuming the time dependence in the form $\exp (-j \omega t)$, the governing equation is derived as:

$$
\begin{aligned}
& \omega^{2}\left(-\left[C_{2}\right]\left\{Q^{\prime \prime}\right\}-\left[C_{1}\right]\left\{Q^{\prime}\right\}+[M]\{Q\}\right) \\
& -\left(\left[E_{1}\right]\left\{Q^{i v}\right\}+\left[E_{2}\right]\left\{Q^{\prime \prime \prime}\right\}+\left[E_{3}\right]\left\{Q^{\prime \prime}\right\}+\left[E_{4}\right]\left\{Q^{\prime}\right\}+\left[E_{5}\right]\{Q\}\right)=0 .
\end{aligned}
$$

The generalized displacement-traction vector $\{Q\}$ appearing above is the assembly of $\{q\}$ for all sublayers, and the matrices $\left[C_{1}\right],\left[C_{2}\right],[M]$, and $\left[E_{1}\right]$ through $\left[E_{5}\right]$ 
are defined in Appendix C. Note that the matrices $[M],\left[C_{2}\right],\left[E_{1}\right],\left[E_{3}\right]$ and $\left[E_{5}\right]$ are symmetric, whereas $\left[C_{1}\right],\left[E_{2}\right]$ and $\left[E_{4}\right]$ are antisymmetric. It is seen that $\{Q\}$ satisfies a fourth order homogeneous ordinary differential equation in $x$.

A solution to equation (2.58) can be assumed in the form

$$
\{Q\}=\left\{Q_{0}\right\} \exp [j(k x-\omega t)]
$$

where $\left\{Q_{0}\right\}$ represents the amplitude vector. Substitution of equation (2.59) into equation (2.58) leads to the following set of linear homogeneous equations to solve for $\left\{Q_{0}\right\}$ :

$$
\left(k^{4}\left[K_{1}\right]-j k^{3}\left[K_{2}\right]-k^{2}\left[K_{3}\right]+j k\left[K_{4}\right]+\left[K_{5}\right]\right)\left\{Q_{0}\right\}=0
$$

where $\left[K_{1}\right]=\left[E_{1}\right],\left[K_{2}\right]=\left[E_{2}\right],\left[K_{3}\right]=\left[E_{3}\right]+\omega^{2}\left[C_{2}\right],\left[K_{4}\right]=\left[E_{4}\right]+\omega^{2}\left[C_{1}\right]$, and $\left[K_{5}\right]=\left[E_{5}\right]-\omega^{2}[M]$. A nontrivial solution can be obtained by setting the determinant of the coefficient matrix to zero. This results in the dispersion relation to solve for the eigenvalues $k$ for a given $\omega$. In other words, $k$ for a given $\omega$ is found by treating equation (2.60) as a fourth order eigenvalue problem in $k$. Alternatively, equation (2.60) can be written as

$$
\left(\left[K_{\rho}\right]-\omega^{2}\left[M_{\rho}\right]\right)\left\{Q_{0}\right\}=0
$$

where

$$
\begin{aligned}
& {\left[K_{\rho}\right]=k^{4}\left[K_{1}\right]-j k^{3}\left[K_{2}\right]-k^{2}\left[E_{3}\right]+j k\left[E_{4}\right]+\left[E_{5}\right],} \\
& {\left[M_{\rho}\right]=[M]-j k\left[C_{1}\right]+k^{2}\left[C_{2}\right] .}
\end{aligned}
$$

This leads to the standard eigenvalue problem for solving $\omega^{2}$ for a given value of $k$. The approximate wave functions are given by the eigenvectors of equation $(2.60)$ or $(2.61)$. 
It should be noted that the formulation presented in this subsection is also valid when the sublayers have orthotropic or even monoclinic material properties in the local $(X, Y, Z)$ coordinate system. This is due to the fact that: orthotropic or monoclinic material properties in local coordinate system remain as monoclinic when transformed into the global $(x, y, z)$ coordinate system; and in equation (2.4) as well as in the subsequent analysis, material properties appear as monoclinic in $(x, y, z)$ coordinate system. The only requirement is that appropriate material transformation equations should be selected from Appendix A.

Formulation for the analysis of plane strain and the antiplane-strain propagation problems using this stiffness method is not presented here since these cases have been well documented by Datta et. al. (1988).

\subsection{Roots of Dispersion Equation}

Dispersion equations obtained in $\S 2.3$ and $\S 2.4$ can be solved for $k$, for given $\omega$ or alternatively, they can be solved for $\omega$, for a given $k$. Due to physical reasons, only real values are acceptable for $\omega$. For a particular value of $\omega$, dispersion equations will have a finite number of real roots and an infinite number of imaginary and complex roots for $k$. The main interest here is to obtain the frequency spectrum (plot of frequency vs. wavenumber) or the phase velocity spectrum (plot of phase velocity vs. frequency) where phase velocity is defined as the ratio $\omega / k$. The frequency spectrum has three different kinds of branches: real, imaginary and complex (for details see Mindlin, 1960; Datta et al., 1988) corresponding to real, imaginary, and complex roots for $k$, respectively. The real branches represent the propagating modes which dominate the dynamic response of the plate. The imaginary and complex branches represent nonpropagating and evanescent modes, repectively, and these modes decay with $x$. 
Using the stiffness method I, an approximation to the frequency spectrum can be found. The real branches (dominant modes) of the approximate spectrum can be easily obtained by solving the standard (first order) eigenvalue problem given by equation (2.61). A point to be noted here is that for real branches, the matrices $\left[K_{\rho}\right]$ and $\left[M_{\rho}\right]$ are real symmetric and positive definite. However, if imaginary and complex branches of the approximate frequency spectrum are to be obtained, then the fourth order eigenvalue problem defined in equation (2.60), which involves a large amount of computer time and core memory, has to be solved.

For a fixed value of either $\omega$ or $k$, the exact dispersion equations obtained in $\S 2.3$ are transcendental functions of either $k$ or $\omega$. It is possible to find the roots of the these transcendental equations by some search method (see Press et al., 1988). This approach will be computationally formidable since the roots are sparsely scattered. Herein, Muller's method (see Conte and Boor, 1972) is employed to recover the exact roots. Approximate roots obtained from the stiffness method I (or II) are used as initial guesses in the Muller's method. If the roots are required over a given range of $k$ (or $\omega$ ), approximate roots from the stiffness methods are required only at the first step to use as initial guesses. At the next step, $k$ (or $\omega$ ) is changed by a small amount and exact dispersion equation is solved taking the exact roots from the previous step as initial guesses for the current step. The process is repeated until the range of interest is scanned.

\subsection{Numerical Results and Discussion}

In this section, the numerical results for dispersion characteristics of homogeneous and laminated composite plates made up of aligned continuous fiber-reinforced material (graphite-epoxy) are presented. On the assumption that the wavelength is much larger than the fiber diameter and spacing between the fibers, each layer 
(lamina) can be modeled as a transversely isotropic medium with the symmetry axis aligned with the fiber direction (see Datta et al., 1984; Ledbetter et al., 1989). These transversely isotropic elastic constants used in the computations here are (in units of $\left.10^{11} \mathrm{~N} / \mathrm{m}^{2}\right), C_{11}=1.6073, C_{22}=0.1392, C_{12}=0.0644, C_{44}=0.0350$, and $C_{55}=0.0707$. In all numerical results presented in this chapter, the nondimensional frequency, wavenumber, and phase velocity are defined as

$$
\Omega=\frac{\omega H}{2 \sqrt{C_{55} / \rho}} \quad, \quad \gamma=\frac{1}{2} k H \quad, \quad C=\frac{\omega}{k \sqrt{C_{55} / \rho}}
$$

respectively. For simplicity, it is assumed in the case of layered plates that all the layers are of equal thickness.

\subsubsection{Accuracy of Stiffness Method I}

The accuracy of the results obtained by the stiffness method I was tested against the analytical solution for propagation in a homogeneous plate and in several crossply (i.e. adjacent layer fibers are perpendicular to each other) plates. For brevity, only some of the results obtained are presented here. Figure 2.2 shows the results for a homogeneous graphite-epoxy plate for propagation in the fiber direction $\left(0^{\circ}\right.$ direction). For the discretization in the stiffness method I, 8 sublayers of equal thickness were used through the half-thickness of the homogeneous plate. Results for a 3 -layer $0^{\circ} / 90^{\circ} / 0^{\circ}$ and 35 -layer $0^{\circ} / 90^{\circ} / 0^{\circ} \cdots \cdots 0^{\circ} / 90^{\circ} / 0^{\circ}$ cross-ply plates for propagation in the $45^{\circ}$ direction are shown in Figures 2.3 and 2.4, respectively. In the stiffness method I, each layer of the 3-layer plate was divided into 8 sublayers, and each layer of the 35-layer plate was treated as a single sublayer. As pointed out in $\S 2.3$, in the analytical method, there is no need to subdivide layers to obtain dispersion curves (subdivision is required only if values of the wave functions at discrete points are desired). It is seen from Figures 2.2-2.4 that the stiffness method 
I gives results that are in excellent agreement with the analytical solution. In order to further investigate the accuracy of the stiffness method I, a comparison between the stiffness methods I and II was made. Results from the two stiffness methods, obtained by varying the number sublayers in the descretization, are given in Tables 2.1 and 2.2 , for the cases of homogeneous plate and the 3-layer $0^{\circ} / 90^{\circ} / 0^{\circ}$ crossply plate, respectively. Corresponding analytical results are also reported in these tables. It is evident from these results that the stiffness method I yields more accurate results for high frequencies than those yielded by the stiffness method II for the same number of sublayers. This is not unexpected since the stiffness method I involves the continuity of both displacements and stresses, whereas the stiffness method II involves only the continuity of displacements. In the remainder of this chapter, attention will be focussed mainly on the dominant branches, namely, real branches of the frequency spectrum. The stiffness method I will be used to obtain required results for the real branches. The reasons for this choice are that the stiffness method I gives straightforward accurate results (without the need of initial guesses), and, specially, the real branches can be obtained from the first order eigenvalue problem defined in equation (2.61).

\subsubsection{Anisotropy Effects}

The degree of anisotropy in a fiber-reinforced composite plate depends on the orientation of the fibers with respect to the direction of wave propagation. In order to show the effect of anisotropy, the real branches of the frequency spectrum for the homogeneous plate for various propagation directions are presented in Figures 2.5(a)-(d). The effect of anisotropy on the dispersion characteristics is quite pronounced in these figures. Comparison among parts (a), (b), (c) and (d) of Figure 2.5 shows that phase velocities (slopes of $\Omega$ vs. $\gamma$ plot) increase much more steeply 
with increasing frequency for propagation close to the fiber direction than for propagation close to the normal direction of fibers. Figure 2.5(a) shows the strongest anisotropy while Figure 2.5(d) shows the least. The next problem considered was an angle-ply plate with $0^{\circ} / 2 \alpha / 0^{\circ}$ ply lay-out configuration for propagation in the $\alpha$ direction. Figures 2.6(a)-(d) show the results of dispersion curves for different values of $\alpha$. The effect of ply lay-out configuration and hence the anisotropy on the dispersion characteristics can be clearly seen in these figures. Figure 2.6(a) is very close to Figure 2.5(a) and Figure 2.6(d) is close to Figure 2.5(d). This is to be expected, because the coupling between antiplane and in-plane motions are not very strong in these cases. It is also seen that the phase velocities decrease with increasing ply angle, for the same frequency.

\subsubsection{Interface Layer Effects}

With a view to examine the effects of interface layers between adjacent laminae, some results showing the measurable changes in phase velocity dispersion in a crossply laminated plate are presented next. Figures 2.7-2.14 show the variations in phase velocity with frequency in a 19-layer graphite-epoxy cross-ply plate with and without interface layers. The material properties of the interface bond layer in units of $10^{11} \mathrm{~N} / \mathrm{m}^{2}$ are

$$
\begin{aligned}
& C_{11}=C_{22}=C_{33}=0.0865, \\
& C_{12}=C_{13}=C_{23}=0.0475, \\
& C_{44}=C_{55}=C_{66}=0.0195 .
\end{aligned}
$$

Figures 2.7 and 2.8 are for propagation along the $0^{\circ}$ and $90^{\circ}$ directions, respectively, when there are no interface layers. Figures 2.9 and 2.10 show the effects of interface layers when the interface layer thickness is one tenth of a lamina thickness and the ratio of the densities of a lamina and an interface layer is 1.5 . In order to 
show the effect of density of interface layer, the dispersion curves for a density ratio of $2 / 3$ are shown in Figures 2.11 and 2.12. Figures 2.13 and 2.14 show the effect of thickness of interface layer.

It is seen from Figures 2.7 and 2.8 that, at low frequencies, the dispersion for propagation along the $0^{\circ}$ and $90^{\circ}$ directions is the same. However, at high frequencies the dispersions for propagation in these two directions are quite different. Comparison of Figures 2.9 and 2.10 with Figures 2.7 and 2.8 shows that the presence of the interface layers lowers the phase velocities and the cut-off frequencies. This feature may have important implications on ultrasonic characterisation of interface bond layers. Figures 2.11 and 2.12 show that raising the density of the interface layers does not change the dispersion of the first few modes appreciably. However, with increasing frequency the differences between Figures 2.9 and 2.10 and Figures 2.11 and 2.12 become appreciable. Within certain frequency bands, these differences are quite large. Finally, a comparison among Figures 2.9, 2.13, and 2.14 shows that increasing the interface layer thickness significantly lowers the phase velocities and cut-off frequencies, specially at high frequencies.

\subsubsection{Layering Effects}

The effect of number of layers on the dispersion behaviour is considered next. For this purpose, dispersion characteristics of graphite-epoxy laminated plates are investigated. It is assumed that the fibers are oriented at $90^{\circ}$ to one another in adjacent layers and that the layering is symmetric with respect to the midplane of the plate, thus requiring only half-thickness of the plate to be modeled. Fibers next to the middle layer are taken to be in $0^{\circ}$ direction and therefore, the fibers in the middle layer are in $90^{\circ}$ direction (i.e. $\cdots \cdots / 0^{\circ} / 90^{\circ} / 0^{\circ} / \cdots \cdots$ configuration). The number of layers (laminae) is varied from 3 to 39 . It is found that when 
this number is sufficiently large, the results obtained can be predicted by using an effective modulus theory (Postma, 1955; Yeo, 1983). This is illustrated in Figures 2.15-2.23. Figures $2.15,2.17,2.19$, and 2.21 show the variations of phase velocity with frequency for propagation in the $0^{\circ}$ direction in a plate with $3,21,27$, and 35 layers, respectively. It is seen that the dispersion behavior changes considerably with the number of layers when the number of layers considered is small. However, as the number of layers becomes sufficiently large the change is not noticeable except at high frequencies for higher modes (see Figures 2.19 and 2.21). A similar feature is seen in Figures 2.16, 2.18, 2.20, and 2.22 for propagation in the $90^{\circ}$ direction. It is also noticed that the dispersion behaviour depicted in Figures 2.21 and 2.22 for a 35-layer plate are remarkably close. This suggests that the plate can be modeled as homogeneous with some effective modulii. In fact, this is seen from Figure 2.23, which shows the predictions of dispersion characteristics obtained using effective modulii calculated in the manner presented by Postma for periodic isotropic layers and generalized for orthotropic layers by Yeo. For cross-ply plates there are six independent effective elastic constants. Their expressions are given by

$$
\begin{aligned}
& C_{11}^{e}=\frac{2 C_{33}\left(C_{11}+C_{33}\right)-\left(C_{13}-C_{23}\right)^{2}}{2 C_{33}}=C_{22}^{e}, \\
& C_{13}^{e}=\frac{1}{2}\left(C_{23}+C_{13}\right)=C_{23}^{e}, \\
& C_{33}^{e}=C_{33}, \\
& C_{12}^{e}=\frac{4 C_{12} C_{33}+\left(C_{13}-C_{23}\right)^{2}}{4 C_{33}} \\
& C_{66}^{e}=C_{66}, \\
& C_{44}^{e}=C_{55}^{e}=\frac{2 C_{44} C_{55}}{C_{44}+C_{55}}
\end{aligned}
$$

where $C_{i j}$ are elastic constants of the $0^{\circ}$ lamina and $C_{i j}^{e}$ are effective elastic modulii for the entire plate. Thus, using the particular properties considered here, the effective modulii of the plate in units of $10^{11} \mathrm{~N} / \mathrm{m}^{2}$ are 


$$
\begin{aligned}
& C_{11}^{e}=0.8732, \quad C_{13}^{e}=0.0668, \quad C_{12}^{e}=0.0664, \\
& C_{33}^{e}=0.1392, \quad C_{44}^{e}=0.0468, \quad C_{66}^{e}=0.0707 .
\end{aligned}
$$

The fact that the effective modulus method predicts wave propagation characteristics quite well for propagation on other directions is seen from Figures 2.24-2.28. Figures $2.24,2.26$, and 2.28 show the results using the layered model calculation whereas Figures 2.25 and 2.27 show those predicted by the effective modulus model. Note that Figures 2.24 and 2.28 show almost the same behaviour. It should also be noted that the dispersion behaviour for propagation in the $0^{\circ}, 22.5^{\circ}$, and $45^{\circ}$ are quite different.

The fact that a thick composite cross-ply plate with sufficiently large number of layers can be modeled as a homogeneous plate with certain effective properties is not unexpected. The important observation of this systematic study is that even though the dispersion of the first few modes can be predicted by the effective medium approximation, for this approximation to be valid for higher modes the plate must have a threshold number of layers.

\subsection{Concluding Remarks}

A stiffness method based on through-thickness interpolation functions for the displacements that maintain continuity of displacements and tractions at the interfaces between layers has been presented and used to study guided wave propagation in a laminated composite plate. An analytical method which uses the prediction of stiffness method as initial guesses has also been presented. In both methods, the plate can have arbitrary number of layers with distinct material properties and thickness. It is shown that the dispersion behaviour predicted by the stiffness method agree well with the analytical solution. 
It is found that measurable changes in phase velocity are caused by interface layers in laminated composite plates. These changes are quite appreciable at high frequencies and specially in higher modes within certain frequency bands. Thus ultrasonic waves may be used to characterise interface bond layer parameters. Observations made here further suggest that judicious choice of frequency and modes can be made to obtain optimum results in ultrasonic nondestructive evaluation of interface bond layer properties.

It is also shown that the number of layers in a cross-ply laminated plate has a strong influence on the dispersion characteristics of waves when the plate is composed of only a few layers. However, as the number of layers increases, the laminated plate can be modeled as a homogeneous anisotropic plate. This feature may have very important significance in ultrasonic characterisation of mechanical properties in thick laminated composite plates. 


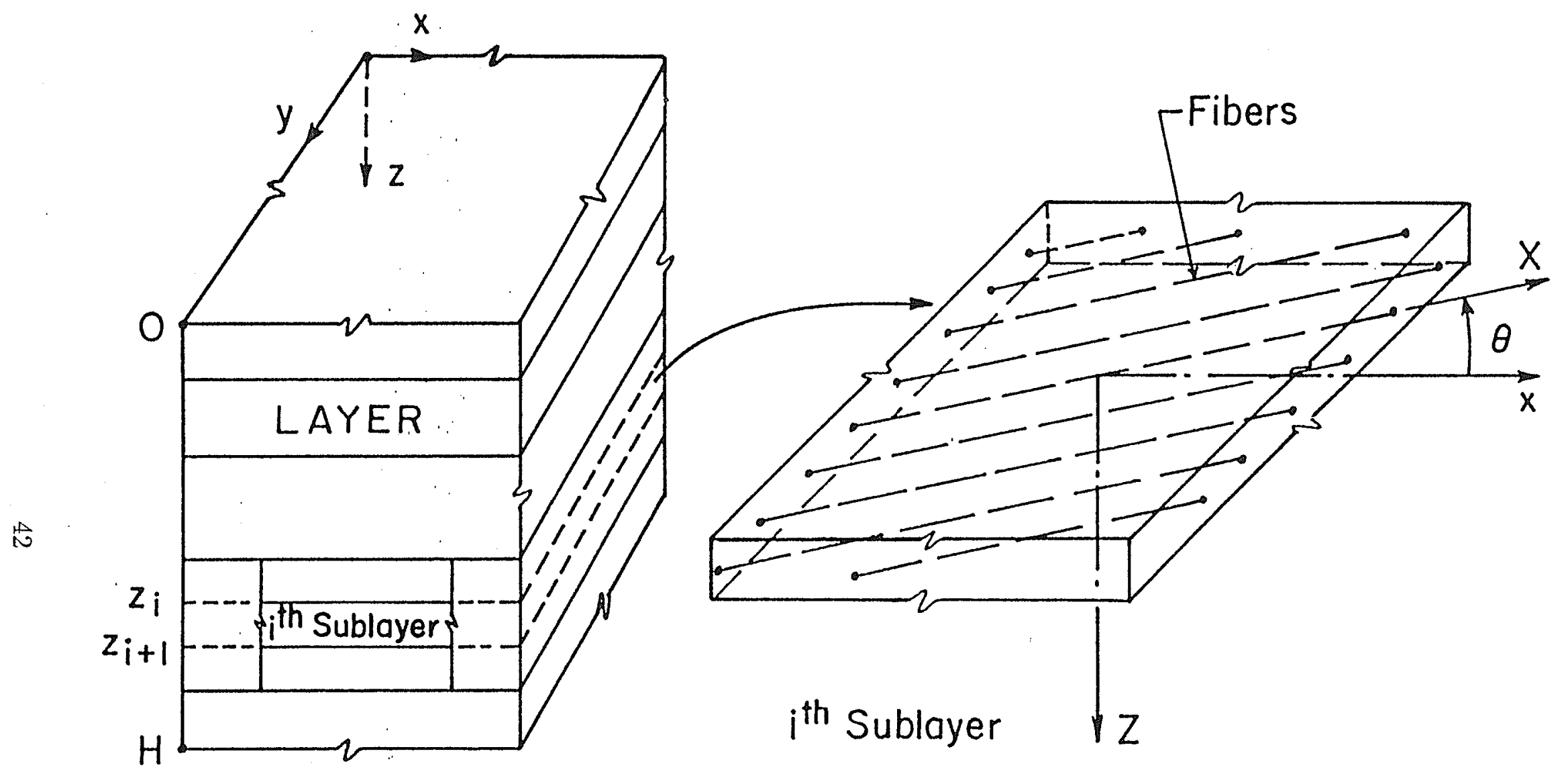

Figure 2.1: Geometry of the layered plate. 
$\Omega$

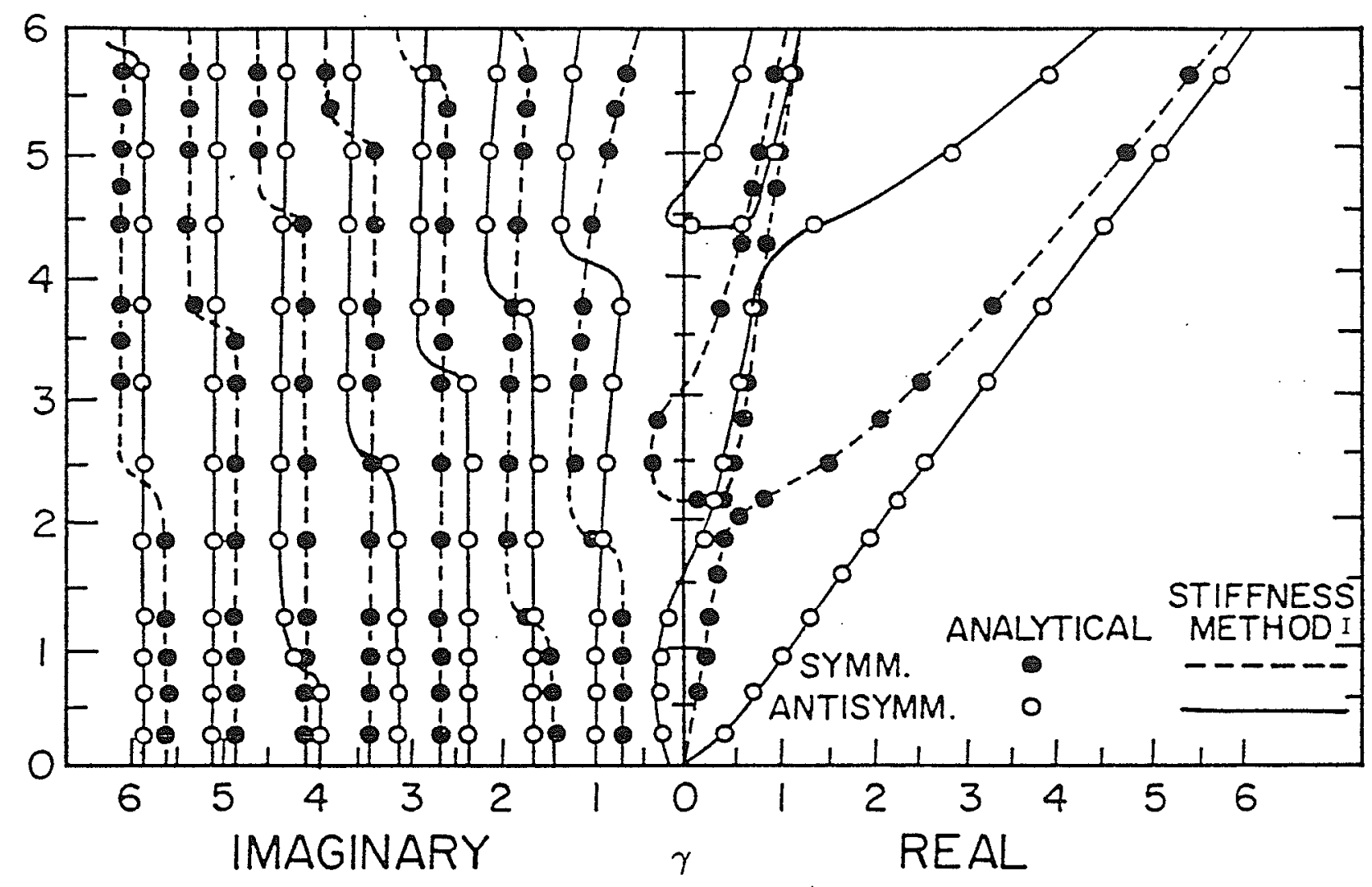

Figure 2.2: Comparison of the results for the dispersion curves from the stiffness method I with analytical results, for a homogeneous graphite-epoxy plate, for propagation in the $0^{\circ}$ direction. 


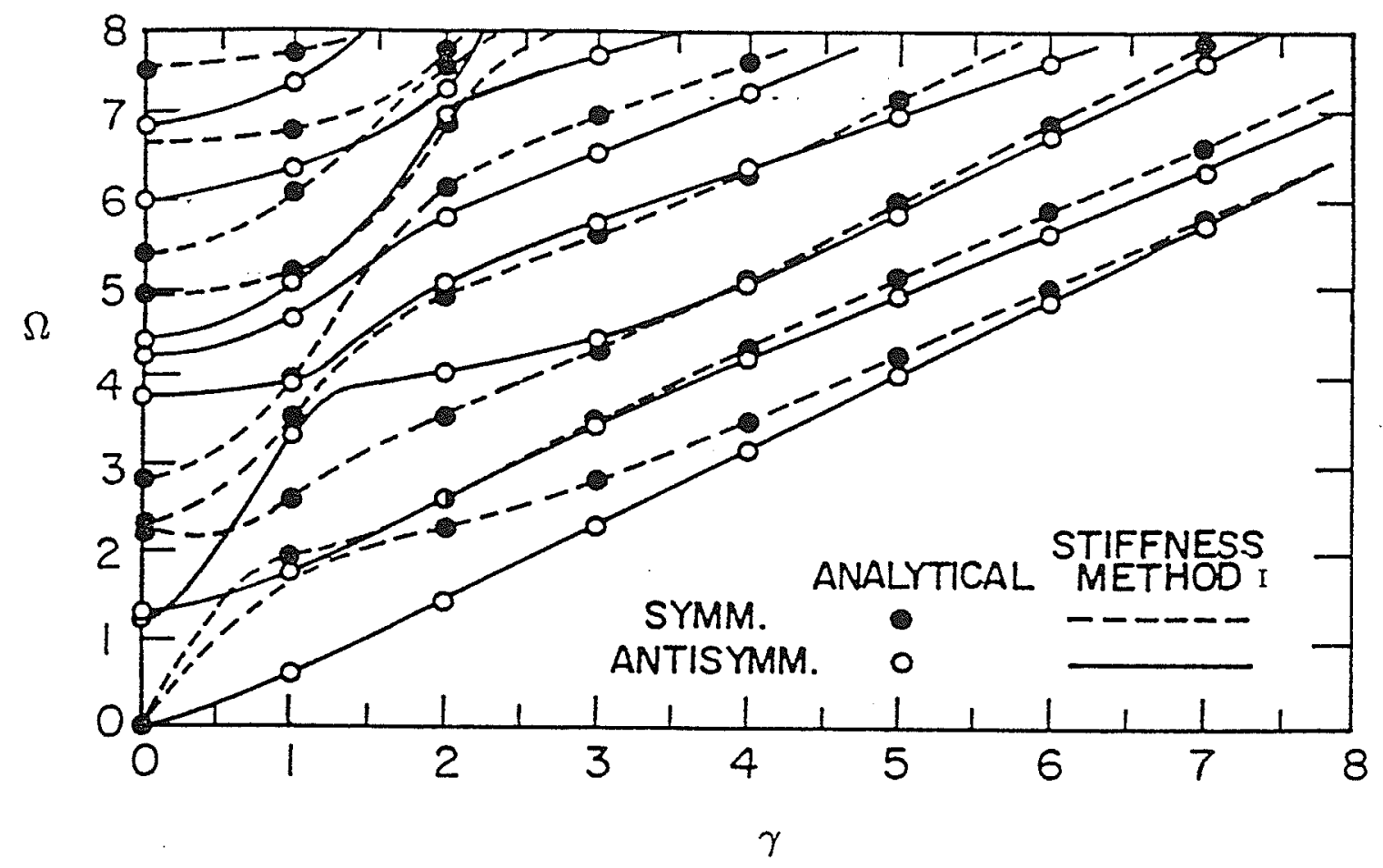

Figure 2.3 : Dispersion curves for a 3-layer cross-ply $\left(0^{\circ} / 90^{\circ} / 0^{\circ}\right)$ graphite-epoxy plate, for propagation in the $45^{\circ}$ direction. 


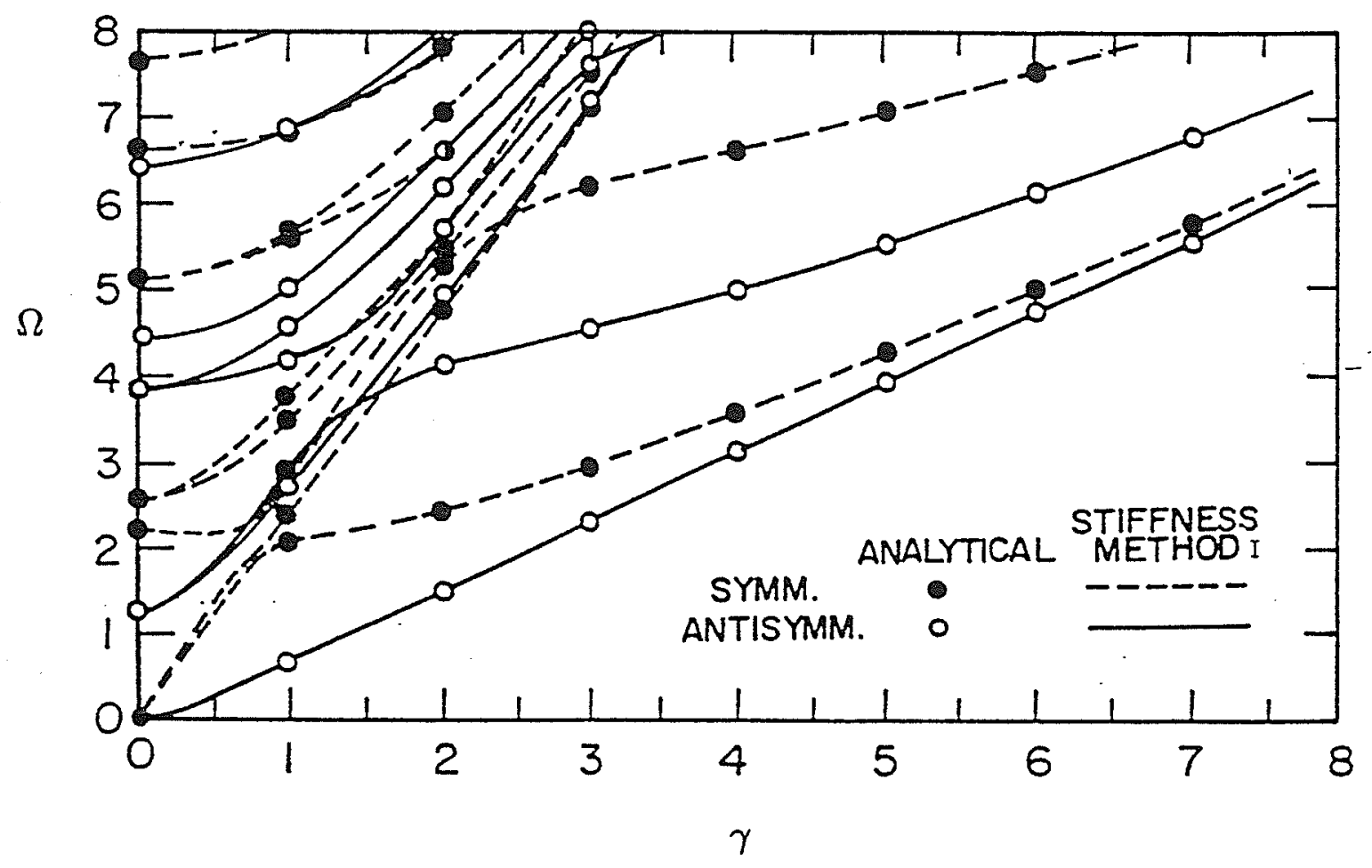

Figure 2.4: Dispersion curves for a 35-layer cross-ply $\left(0^{\circ} / 90^{\circ} / 0^{\circ} / \cdots \cdots / 0^{\circ} / 90^{\circ} / 0^{\circ}\right)$ graphite-epoxy plate, for propagation in the $45^{\circ}$ direction. 

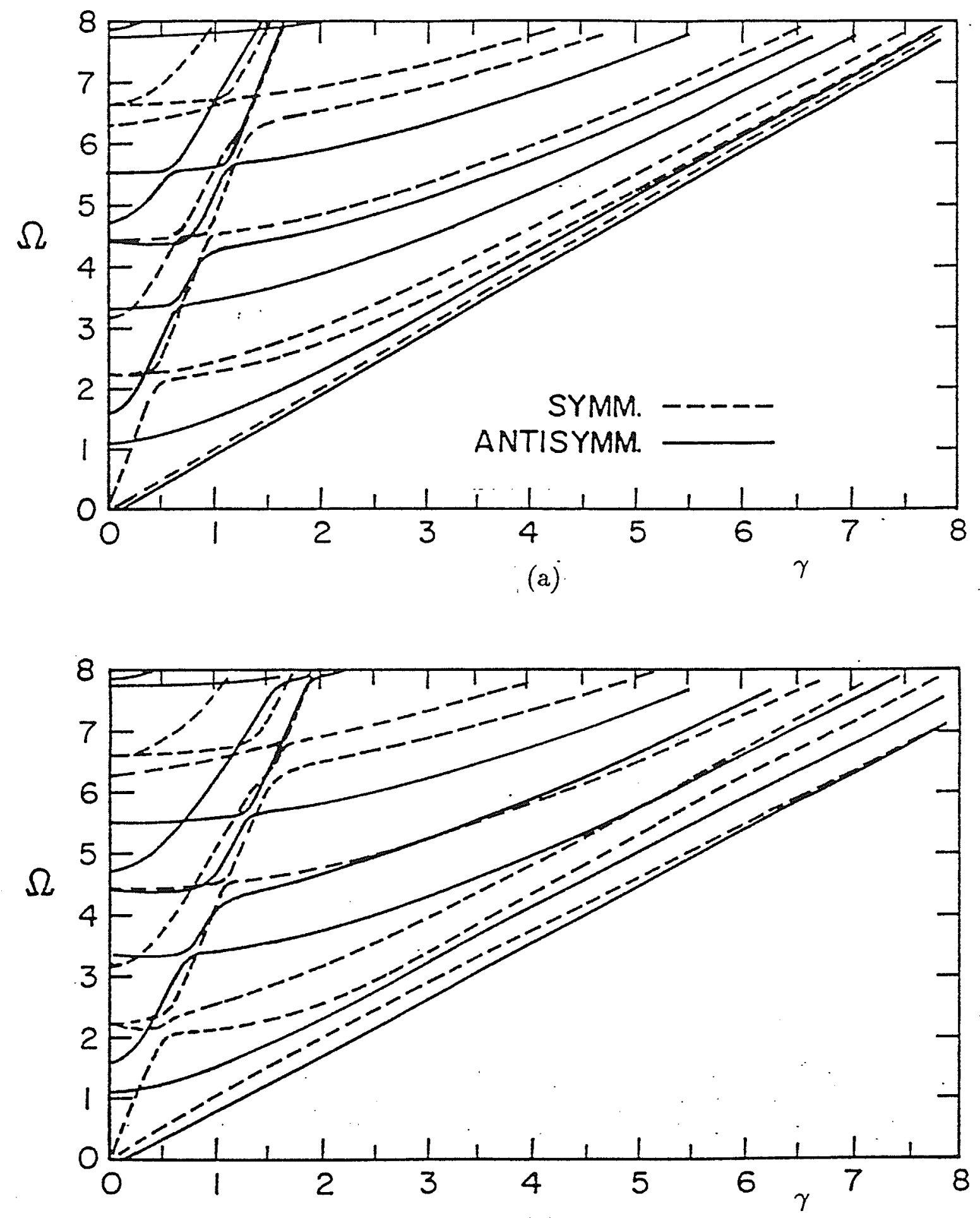

(b)

Figure 2.5 : Dispersion curves for a homogeneous graphite-epoxy plate, for propagation in the:
(a) $5^{\circ}$ direction,
(c) $60^{\circ}$ direction,
(b) $30^{\circ}$ direction,
(d) $85^{\circ}$ direction. 

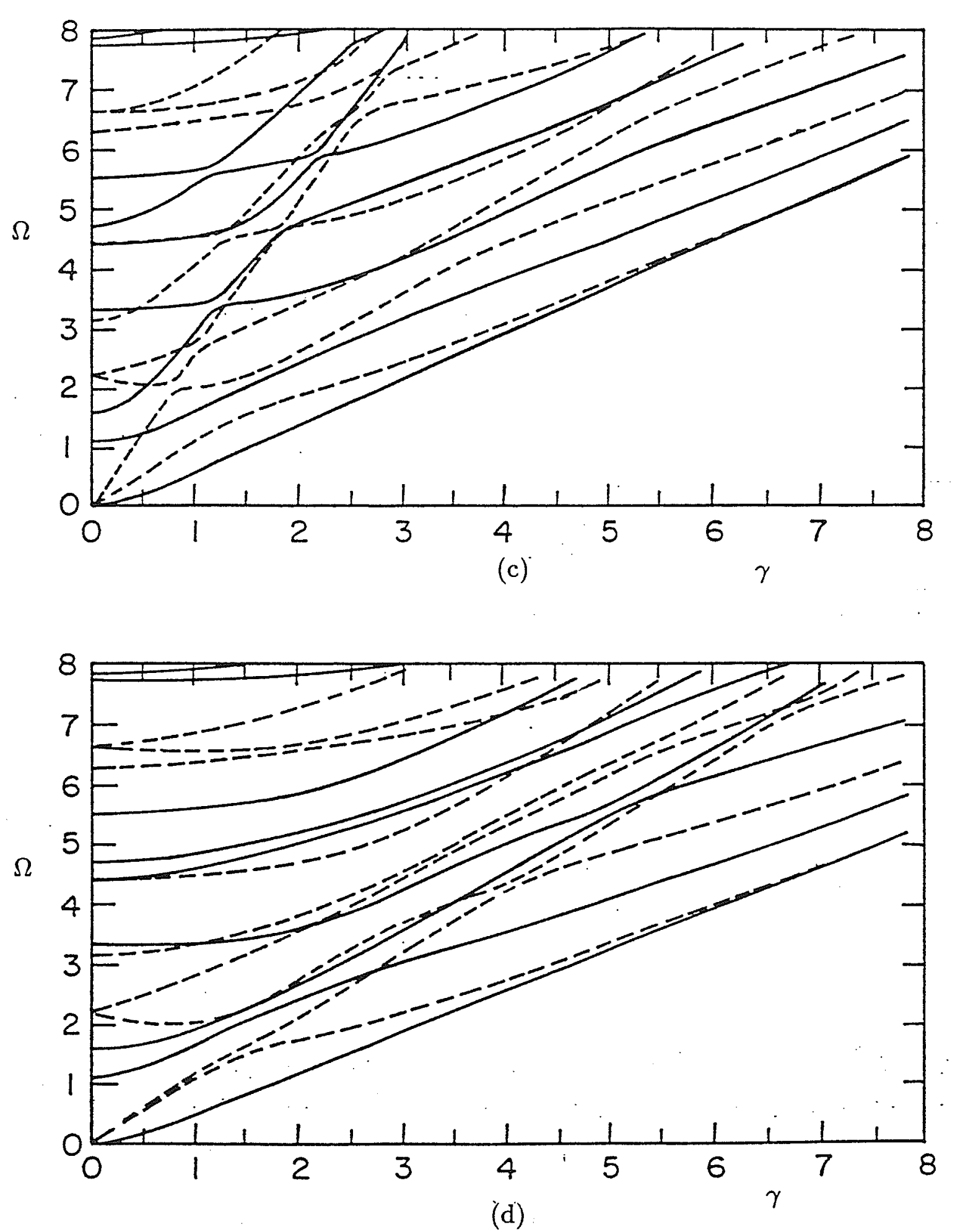

Figure 2.5 : Dispersion curves for a homogeneous graphite-epoxy plate, for propagation in the:
(a) $5^{\circ}$ direction,
(c) $60^{\circ}$ direction,
(b) $30^{\circ}$ direction,
(d) $85^{\circ}$ direction. 


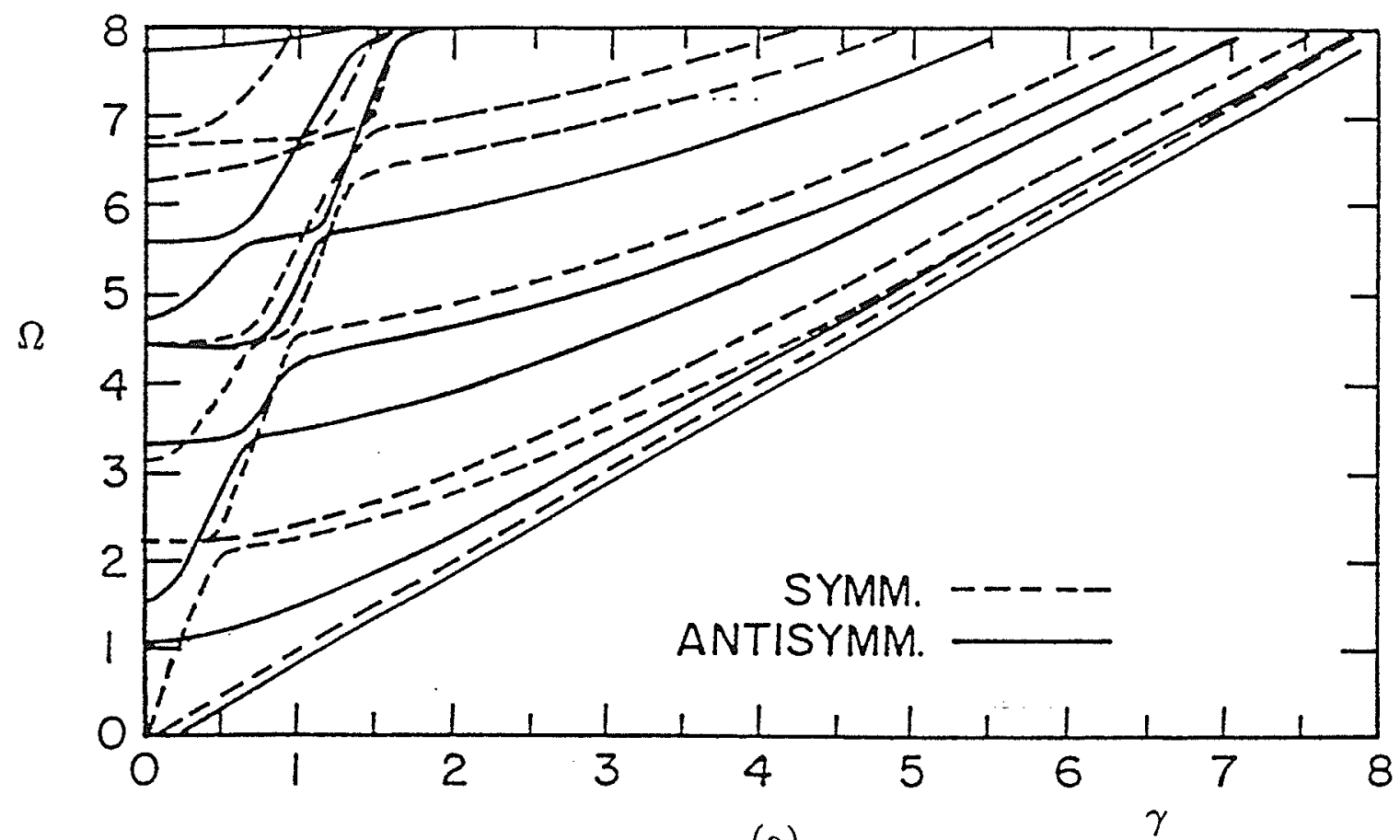

(a)

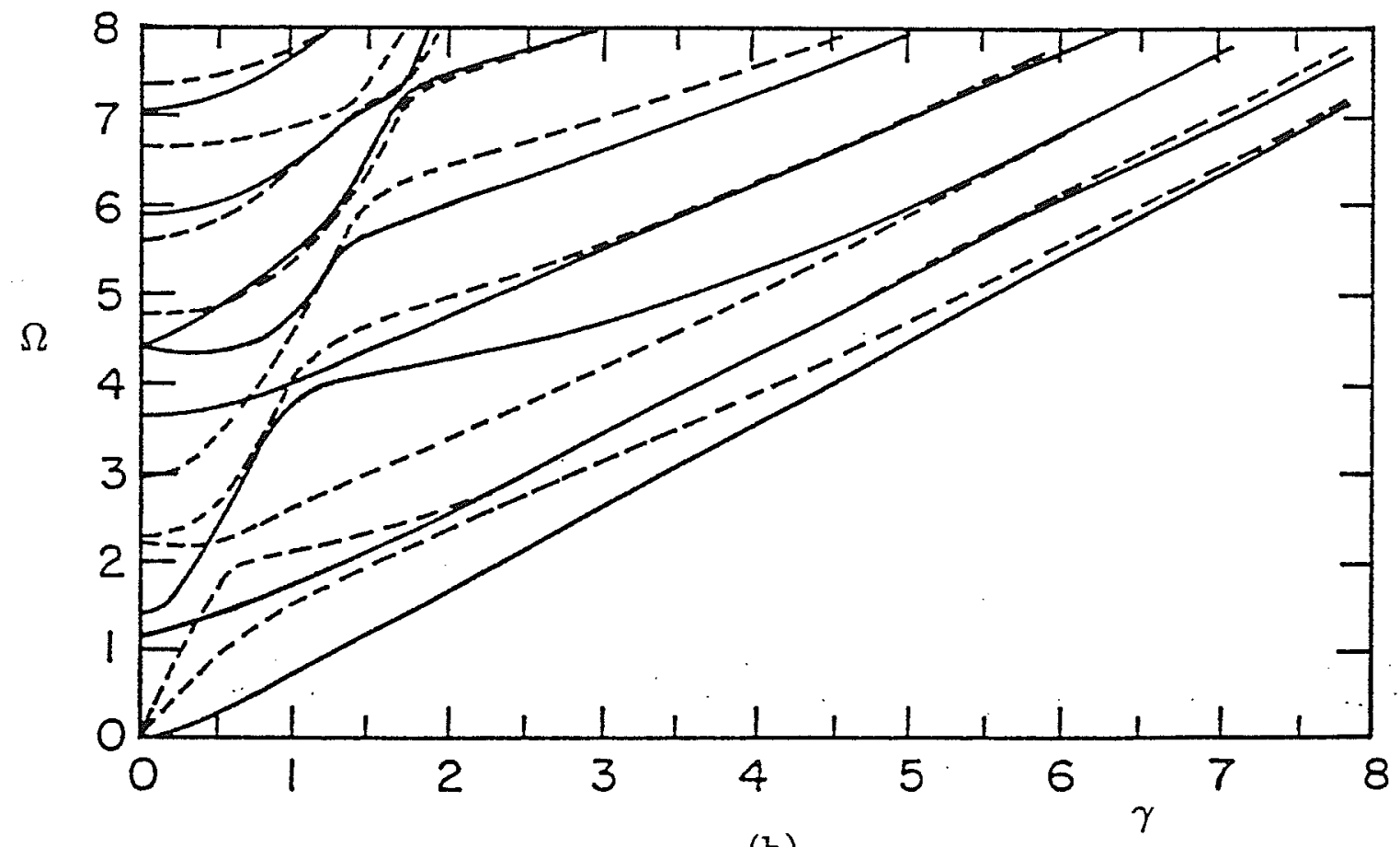

(b)

Figure 2.6 : Dispersion curves for a 3-layer angle-ply $\left(0^{\circ} / 2 \alpha / 0^{\circ}\right)$ graphite-epoxy plate, for propagation in the $\alpha$ direction:
(a) $\alpha=5^{\circ}$,
(b) $\alpha=30^{\circ}$,
(c) $\alpha=60^{\circ}$,
(d) $\alpha=85^{\circ}$. 

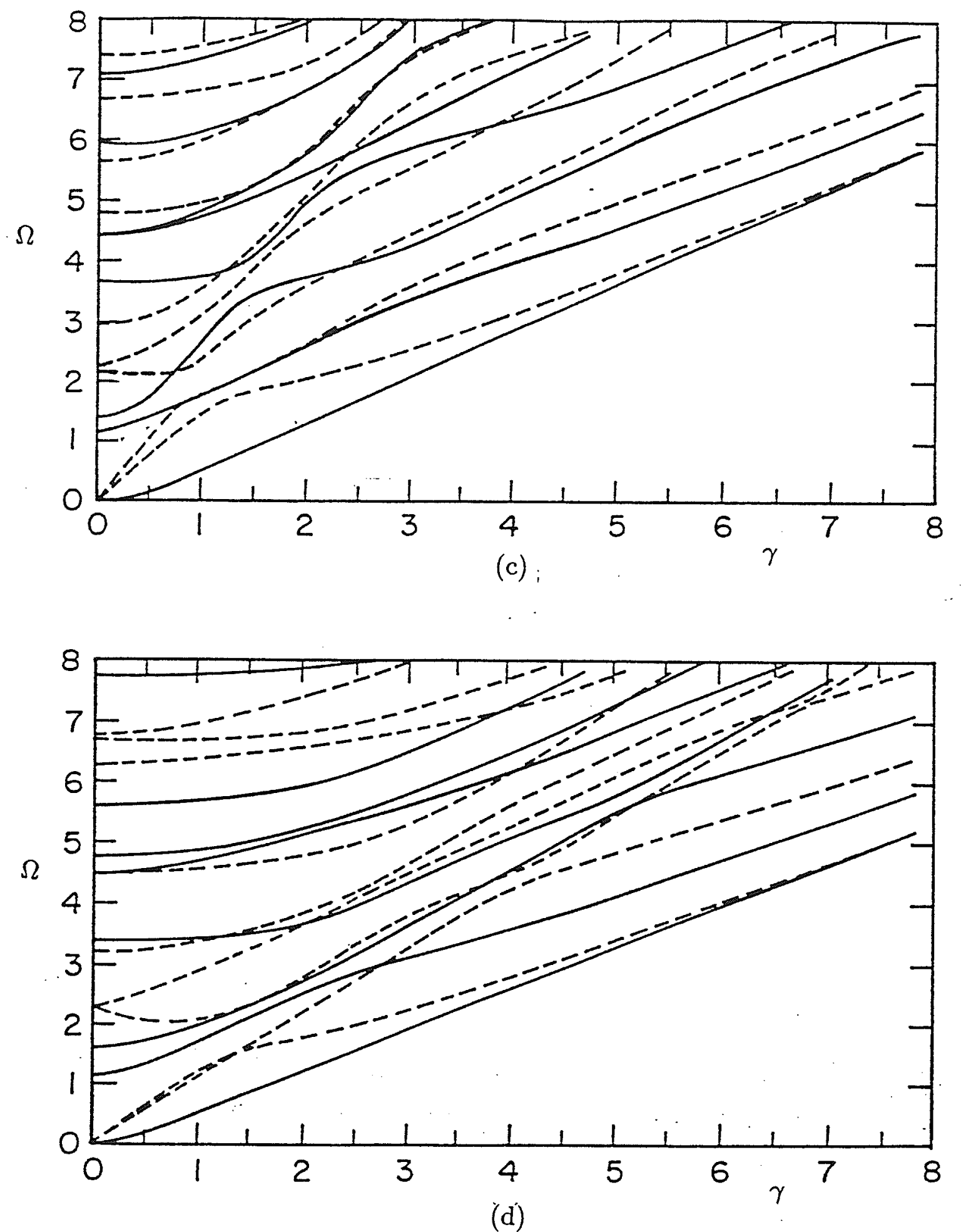

Figure 2.6 : Dispersion curves for a 3-layer angle-ply $\left(0^{\circ} / 2 \alpha / 0^{\circ}\right)$ graphite-epoxy plate, for propagation in the $\alpha$ direction:
(a) $\alpha=5^{\circ}$,
(c) $\alpha=60^{\circ}$,
(b) $\alpha=30^{\circ}$,
(d) $\alpha=85^{\circ}$. 


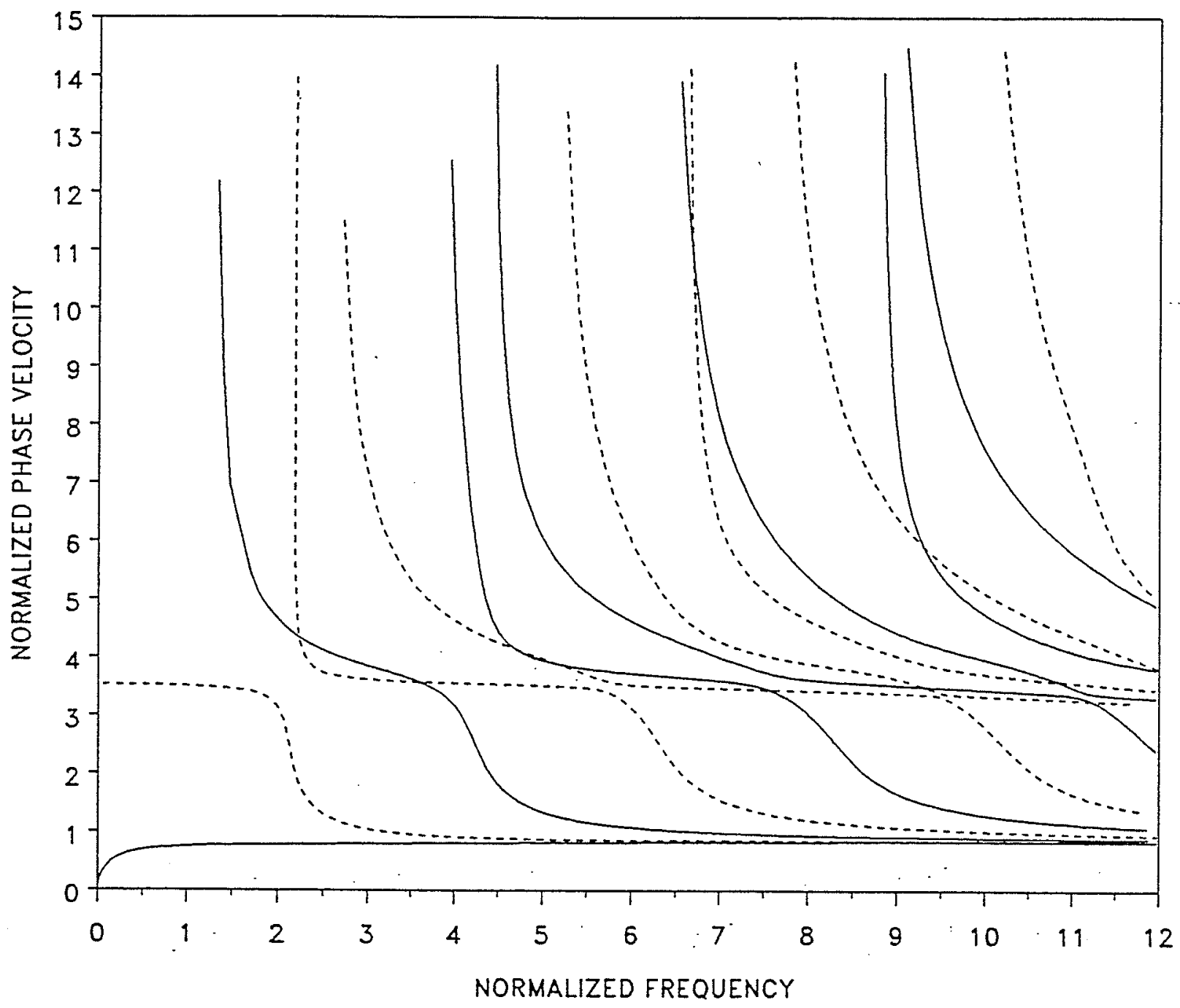

Figure 2.7 : Dispersion of waves in a 19-layer cross-ply $\left(0^{\circ} / 90^{\circ} / 0^{\circ} / \cdots \cdots / 0^{\circ} / 90^{\circ} / 0^{\circ}\right)$ graphite-epoxy plate, for propagation in the $0^{\circ}$ direction:

Symmetric modes - ....; Antisymmetric modes 


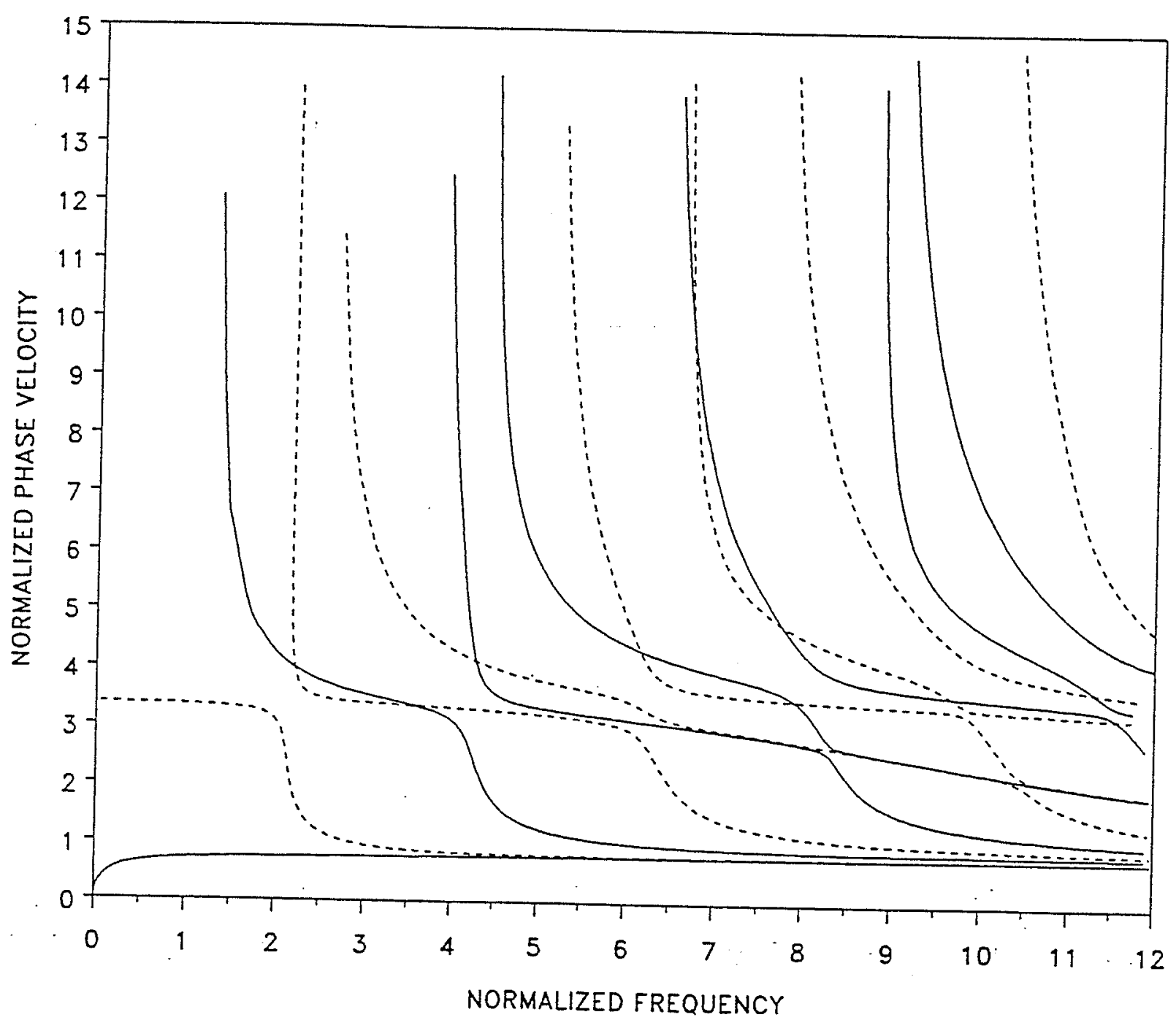

Figure 2.8 : Dispersion of waves in a 19-layer cross-ply $\left(0^{\circ} / 90^{\circ} / 0^{\circ} / \cdots \cdots / 0^{\circ} / 90^{\circ} / 0^{\circ}\right)$ graphite-epoxy plate, for propagation in the $90^{\circ}$ direction: Symmetric modes - .....; Antisymmetric modes 


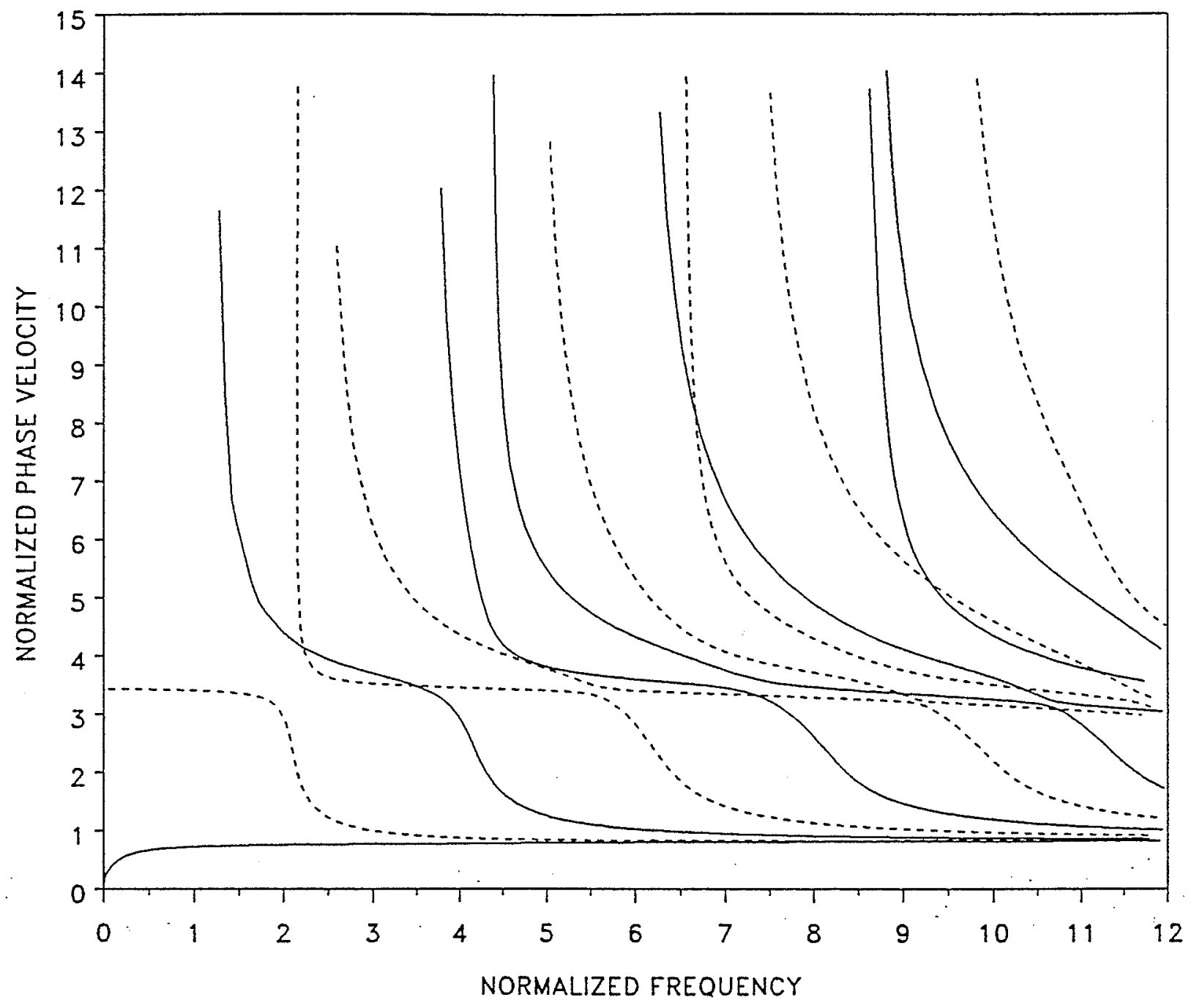

Figure 2.9: Dispersion of waves in a 19-layer cross-ply $\left(0^{\circ} / 90^{\circ} / 0^{\circ} / \cdots \cdots / 0^{\circ} / 90^{\circ} / 0^{\circ}\right)$ graphite-epoxy plate with interface layers between $0^{\circ}$ and $90^{\circ}$ layers: The ratios of the thickness and densities of a layer and an interface are 10 and 1.5 , respectively; Propagation is in the $0^{\circ}$ direction;

Symmetric modes -.... - ; Antisymmetric modes 


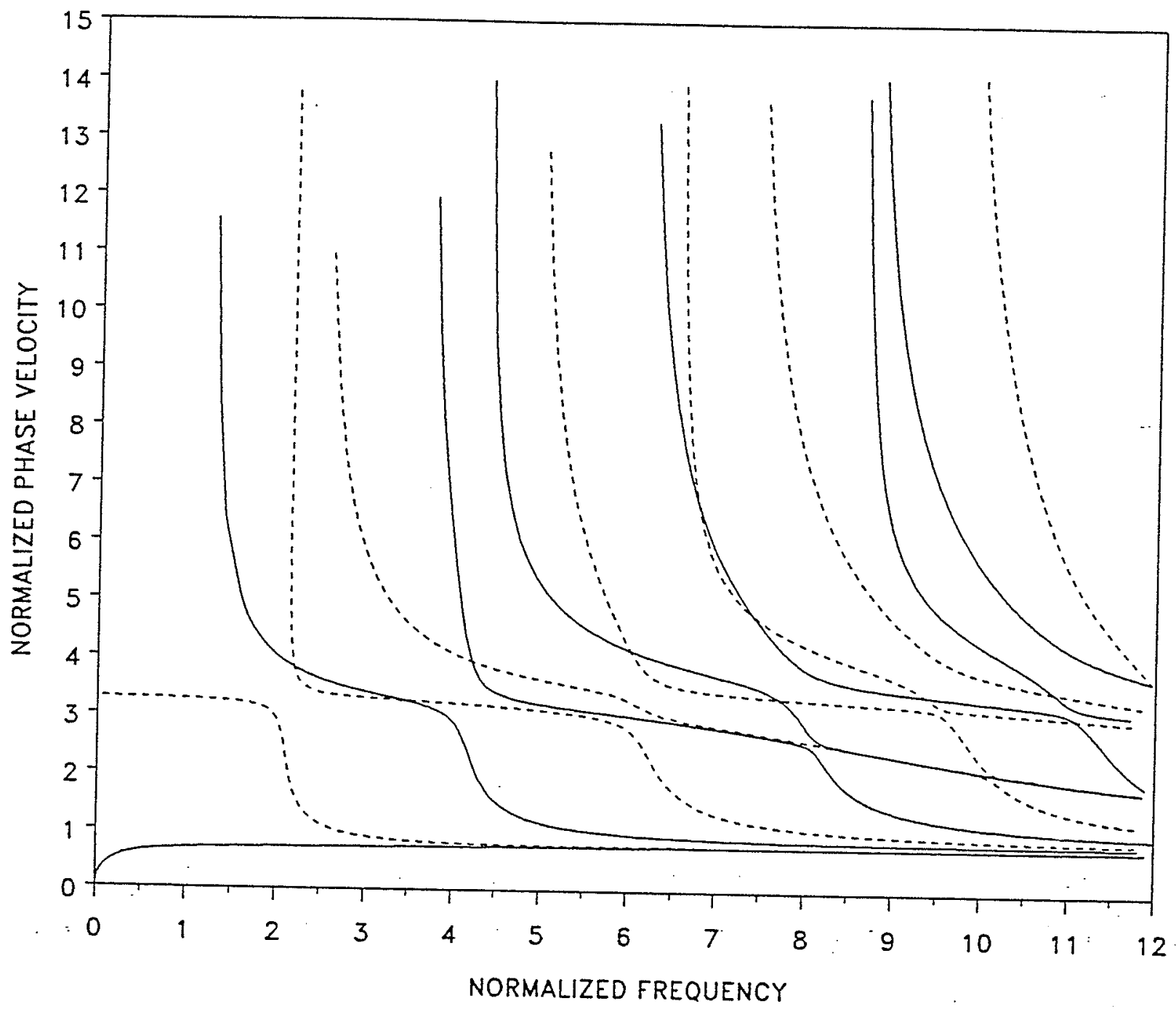

Figure 2.10 : Dispersion of waves in a 19-layer cross-ply $\left(0^{\circ} / 90^{\circ} / 0^{\circ} / \cdots \cdots / 0^{\circ} / 90^{\circ} / 0^{\circ}\right)$ graphite-epoxy plate with interface layers between $0^{\circ}$ and $90^{\circ}$ layers: The ratios of the thickness and densities of a layer and an interface are 10 and 1.5 , respectively; Propagation is in the $90^{\circ}$ direction;

Symmetric modes - ... - ; Antisymmetric modes 


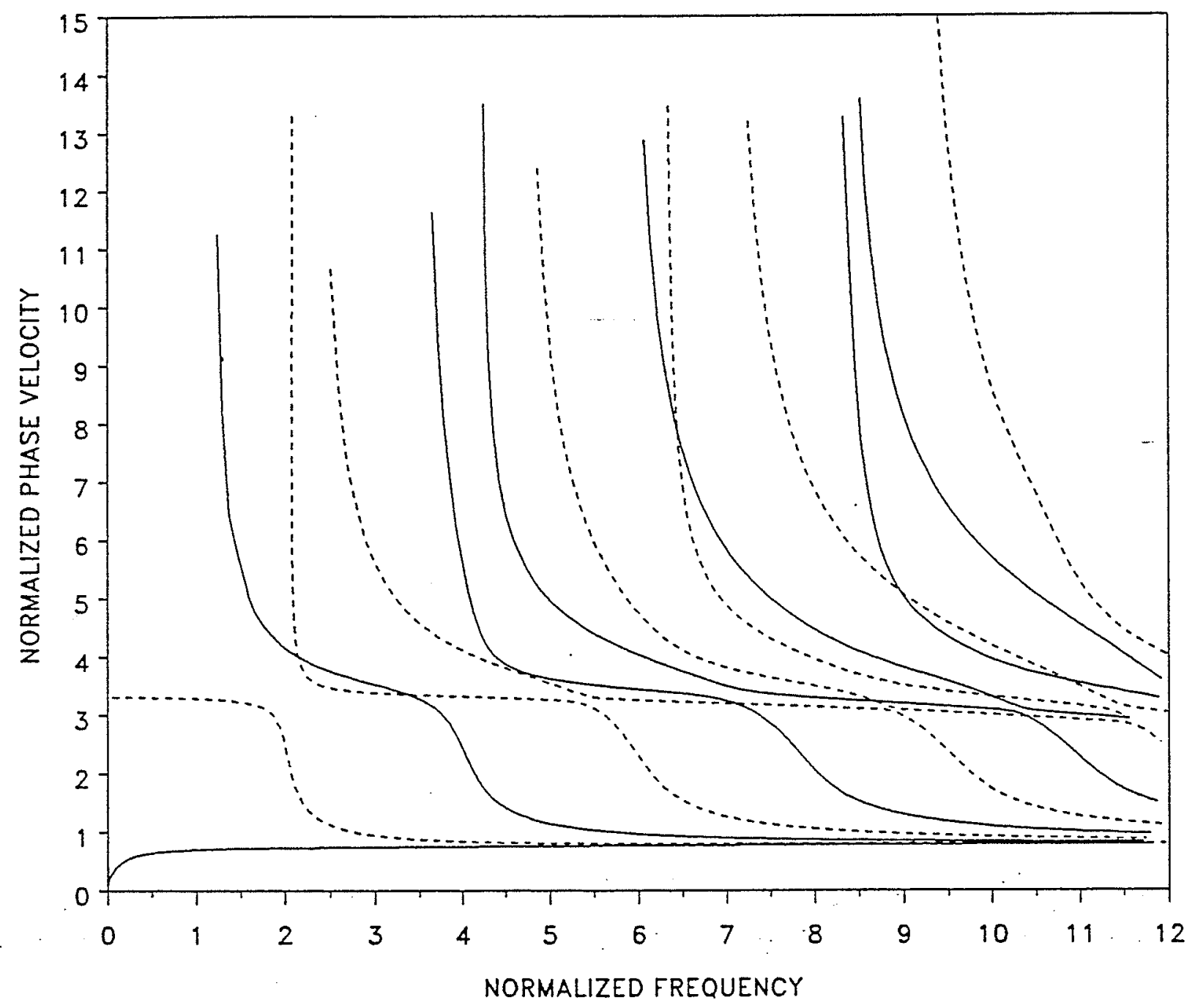

Figure 2.11 : Same as in Figure 2.9, but the density ratio is 2/3: Symmetric modes - . . . . ; Antisymmetric modes 


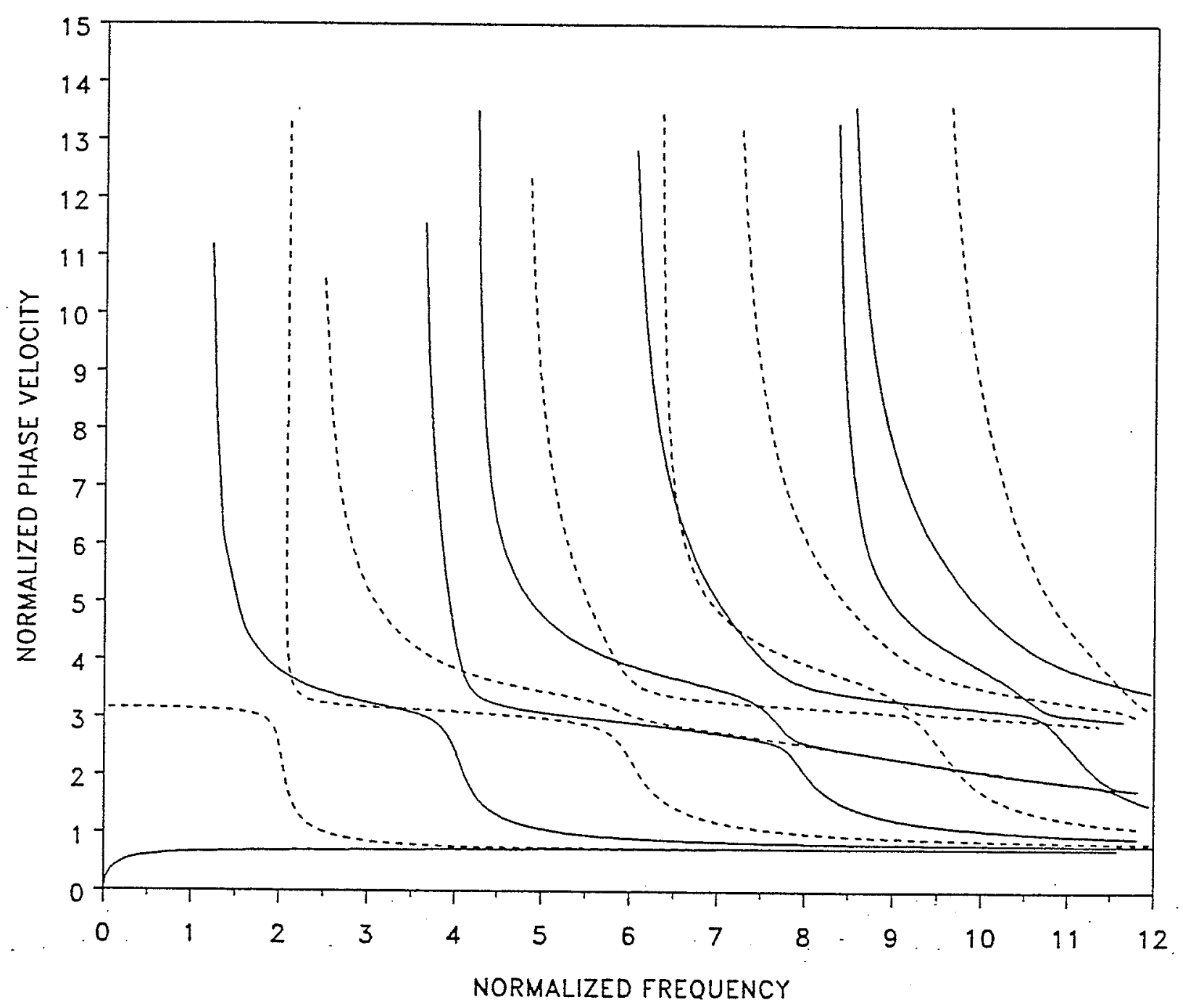

Figure 2.12: Same as in Figure 2.10, but the density ratio is 2/3:

Symmetric modes - . . . - ; Antisymmetric modes 


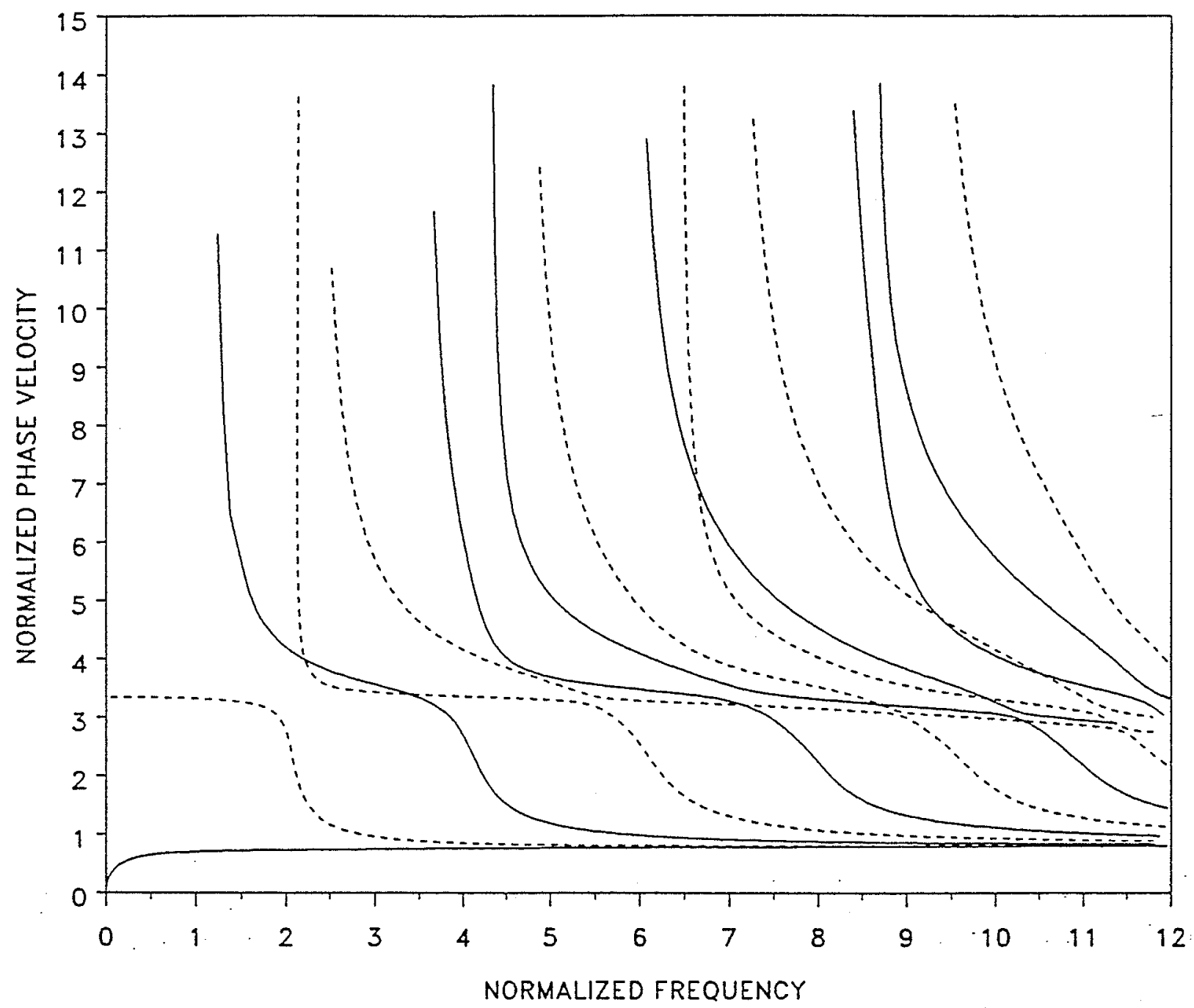

Figure 2.13 : Same as in Figure 2.9, but the thickness ratio is 5: Symmetric modes -..... - Antisymmetric modes 


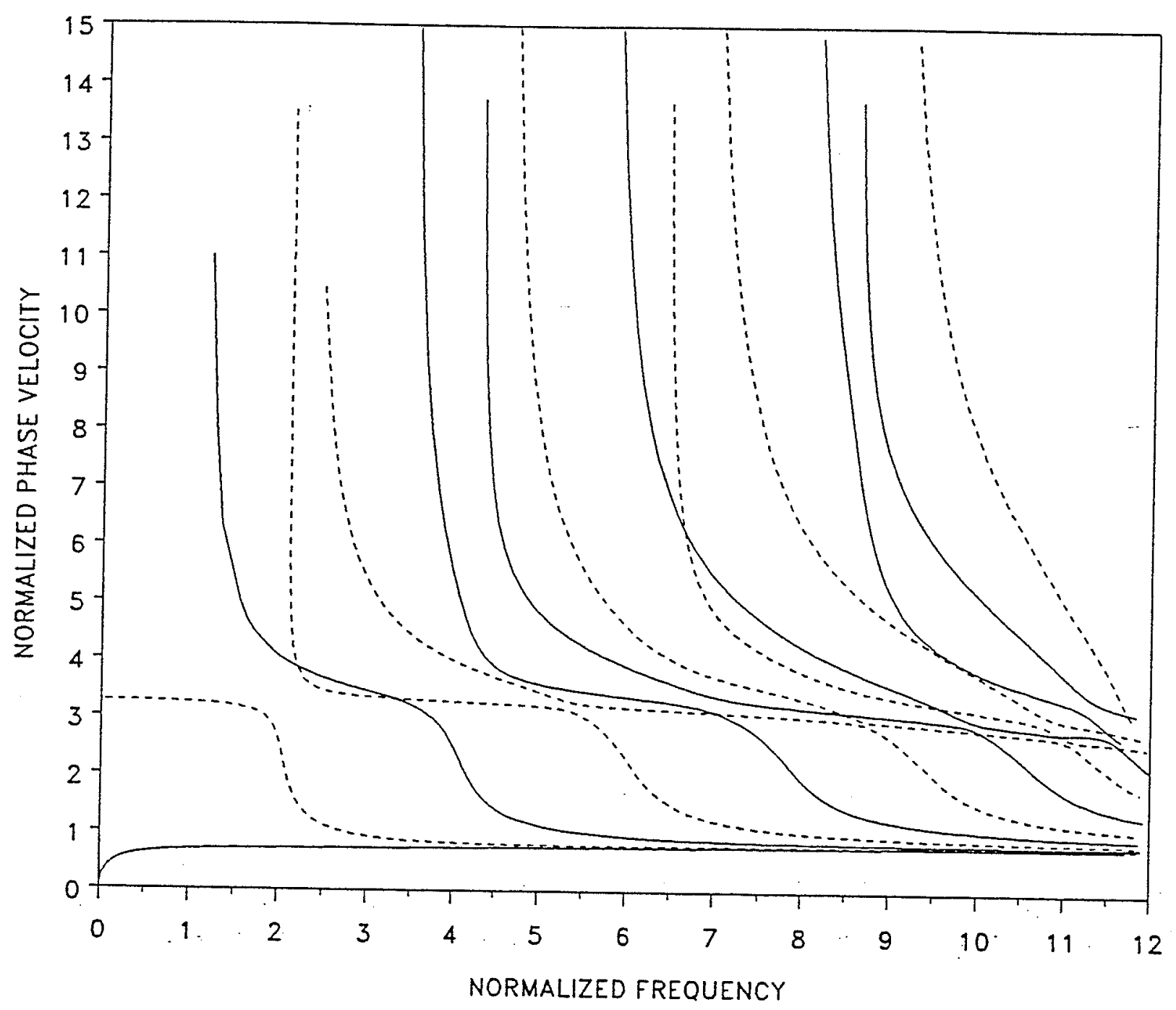

Figure 2.14: Same as in Figure 2.9, but the thickness ratio is 10/3: Symmetric modes - .... . ; Antisymmetric modes 


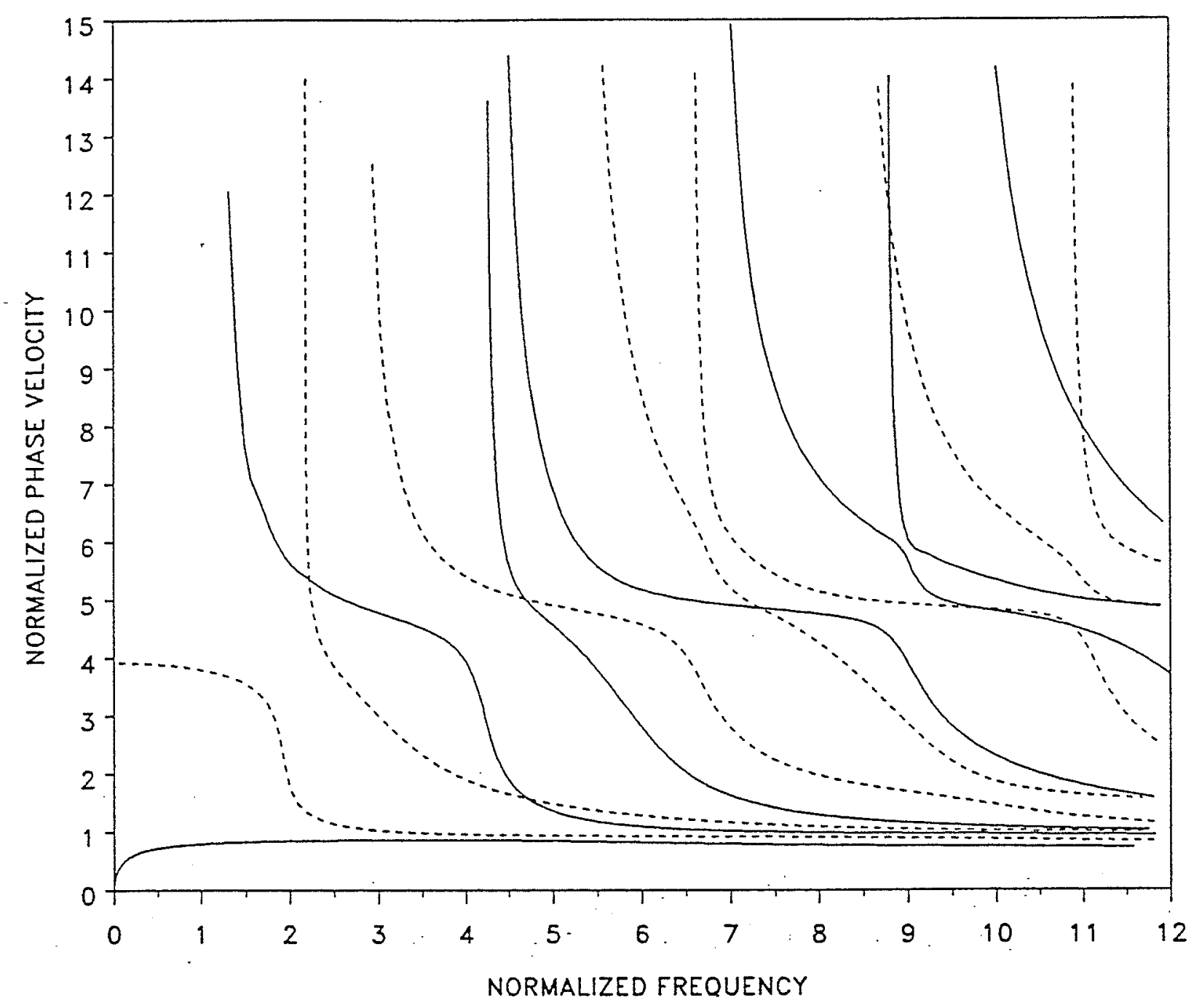

Figure 2.15: Dispersion of waves in a 3-layer cross-ply $\left(0^{\circ} / 90^{\circ} / 0^{\circ}\right)$ graphite-epoxy plate, for propagation in the $0^{\circ}$ direction:

Symmetric modes -..... - Antisymmetric modes - 


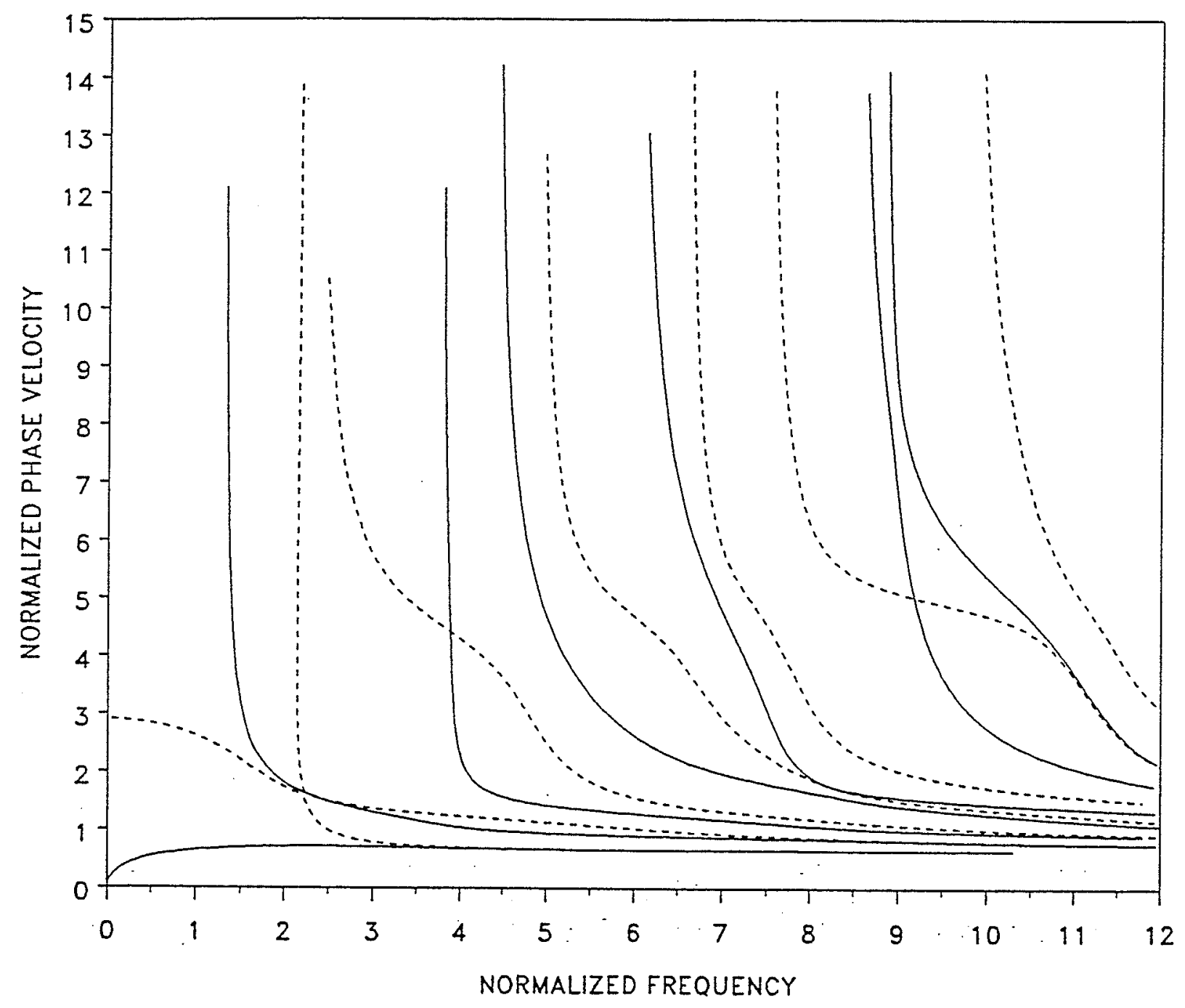

Figure 2.16 : Dispersion of waves in a 3-layer cross-ply $\left(0^{\circ} / 90^{\circ} / 0^{\circ}\right)$ graphite-epoxy plate, for propagation in the $90^{\circ}$ direction:

Symmetric modes - - . - - ; Antisymmetric modes - 


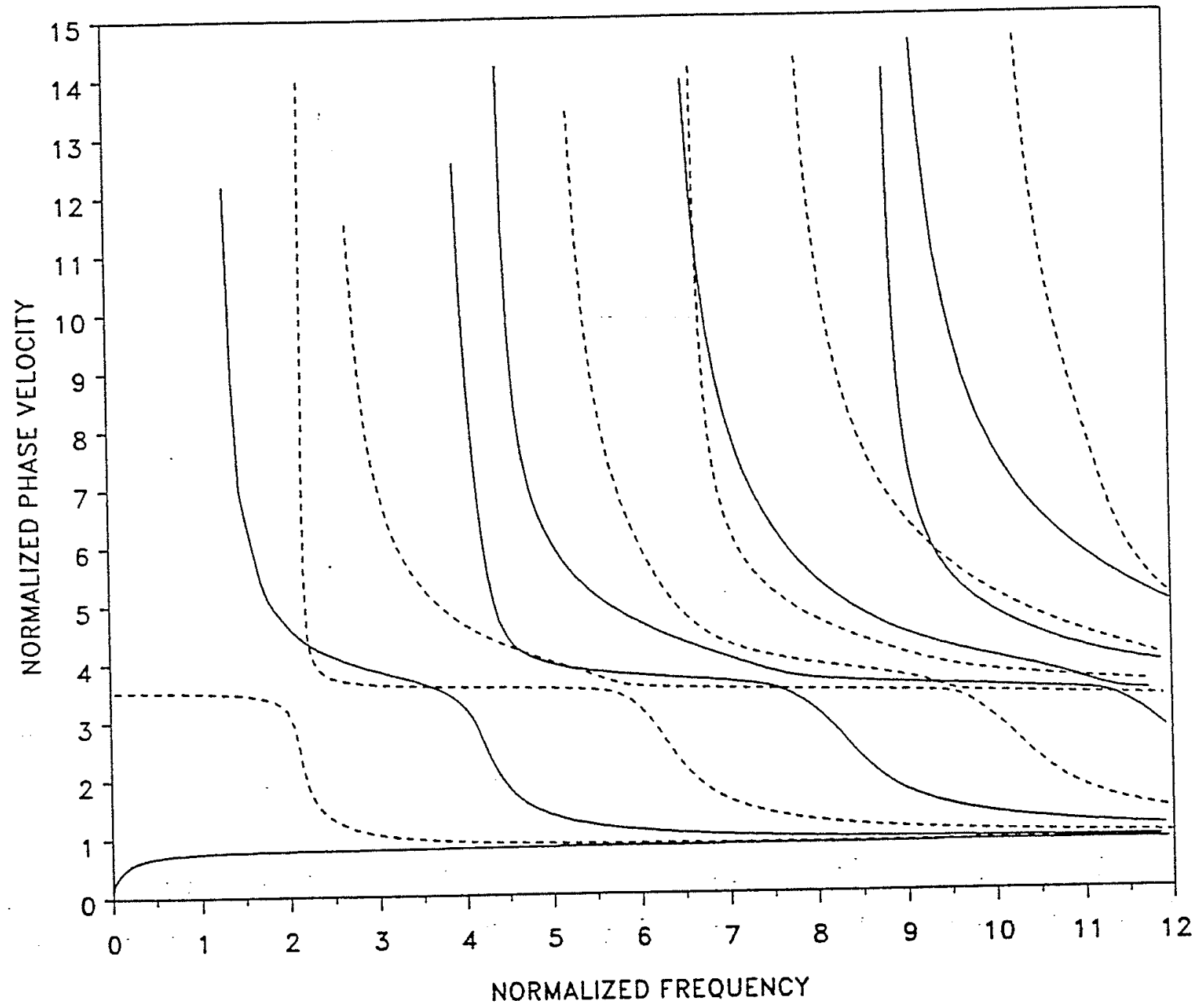

Figure 2.17: Dispersion of waves in a 21-layer cross-ply $\left(0^{\circ} / 90^{\circ} / 0^{\circ} / \cdots \cdots / 0^{\circ} / 90^{\circ} / 0^{\circ}\right)$ graphite-epoxy plate, for propagation in the $0^{\circ}$ direction: Symmetric modes -.....; Antisymmetric modes 


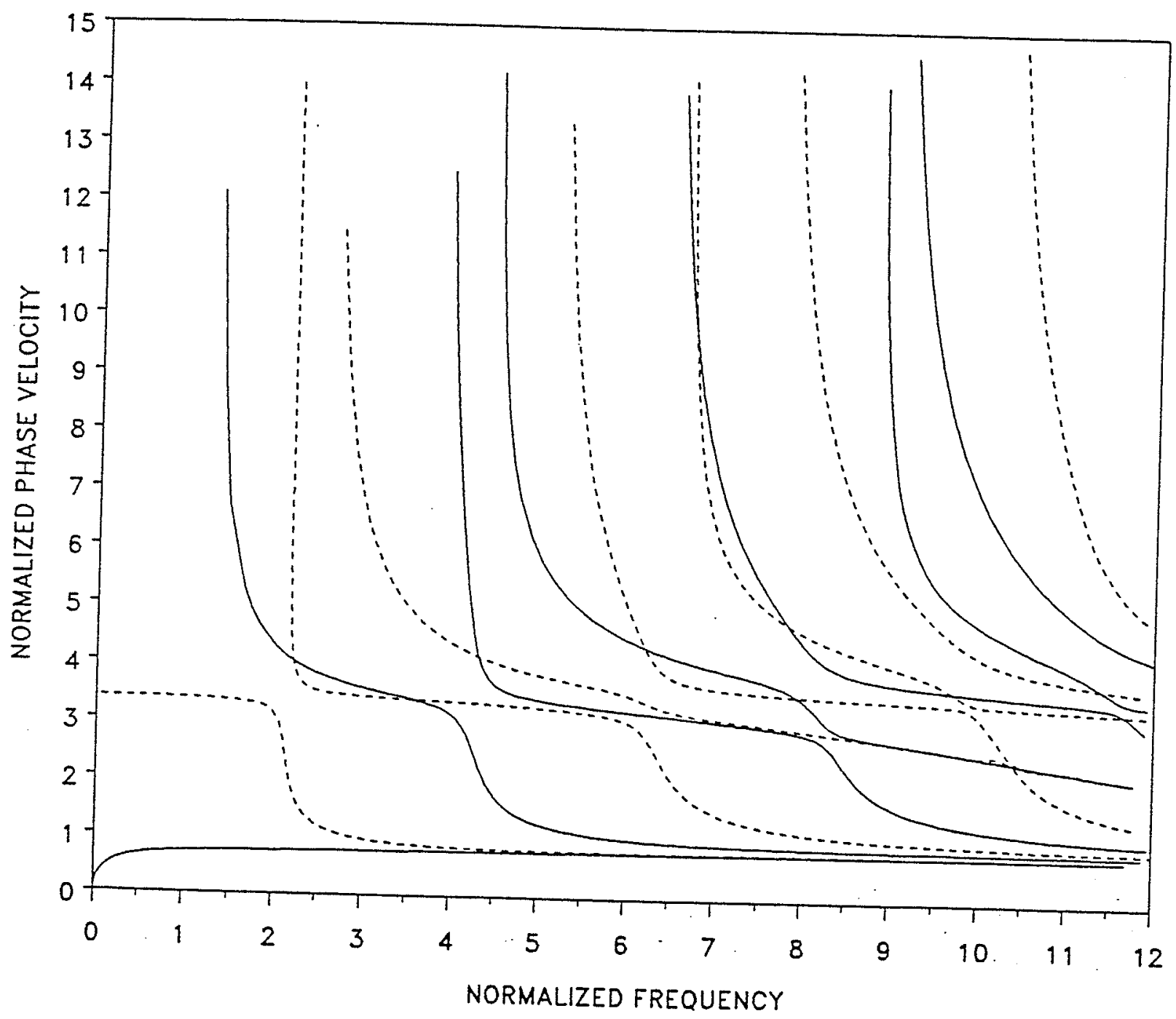

Figure 2.18: Dispersion of waves in a 21-layer cross-ply $\left(0^{\circ} / 90^{\circ} / 0^{\circ} / \cdots \cdots / 0^{\circ} / 90^{\circ} / 0^{\circ}\right)$ graphite-epoxy plate, for propagation in the $90^{\circ}$ direction:

Symmetric modes - ..... ; Antisymmetric modes - 


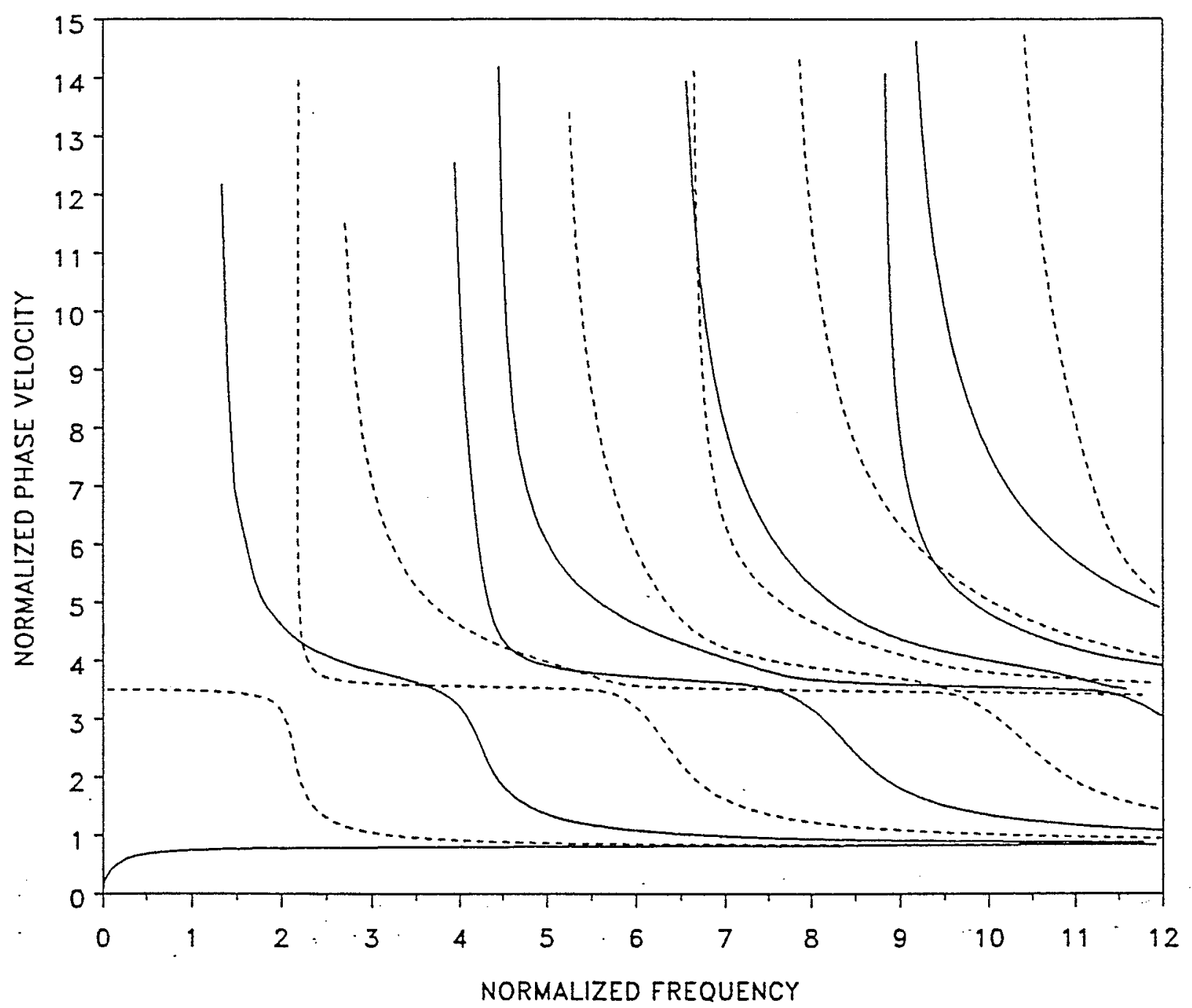

Figure 2.19: Dispersion of waves in a 27-layer cross-ply $\left(0^{\circ} / 90^{\circ} / 0^{\circ} / \cdots \cdots / 0^{\circ} / 90^{\circ} / 0^{\circ}\right)$ graphite-epoxy plate, for propagation in the $0^{\circ}$ direction: Symmetric modes - . - . - ; Antisymmetric modes - 


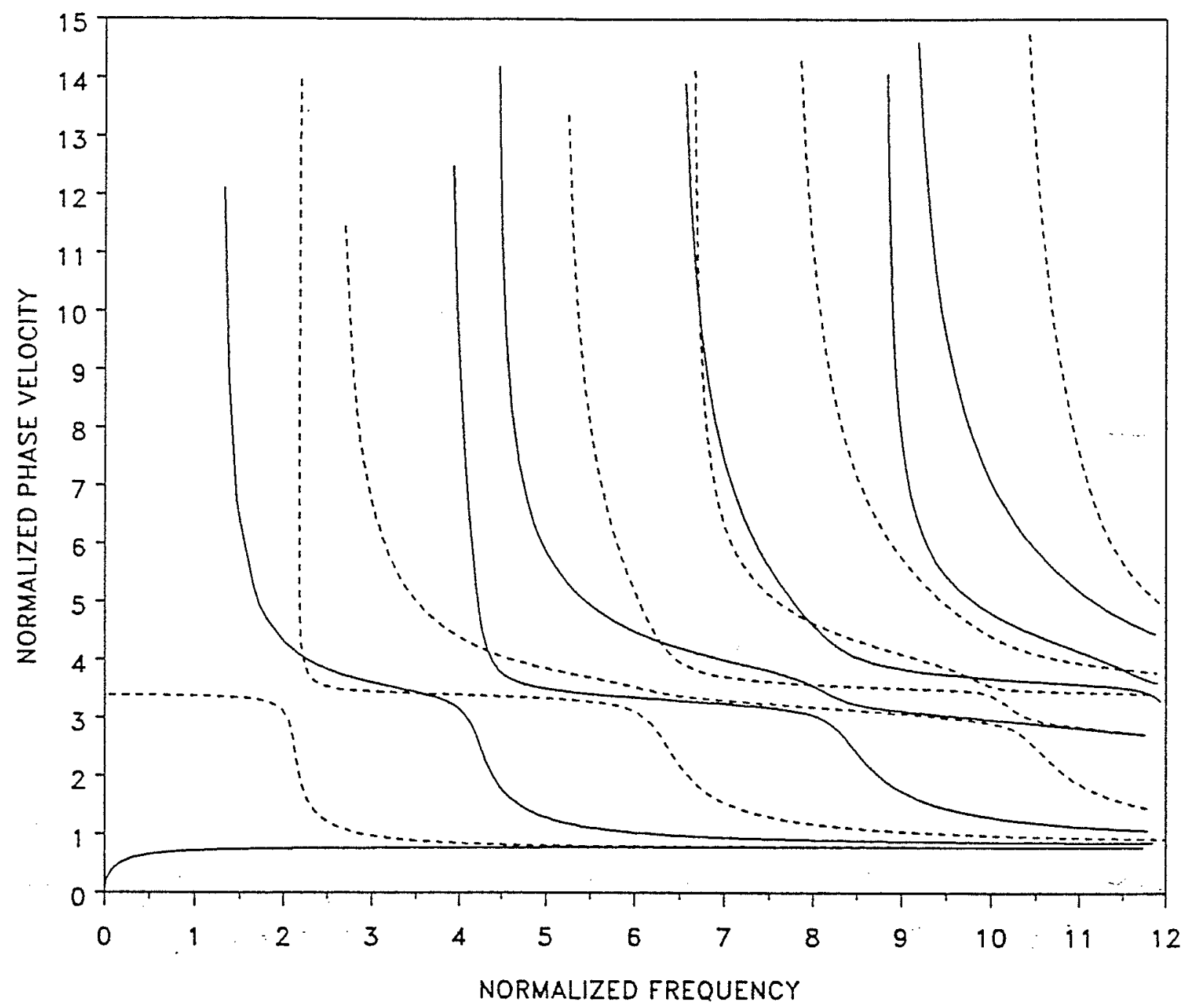

Figure 2.20: Dispersion of waves in a 27-layer cross-ply $\left(0^{\circ} / 90^{\circ} / 0^{\circ} / \cdots \cdots / 0^{\circ} / 90^{\circ} / 0^{\circ}\right)$ graphite-epoxy plate, for propagation in the $90^{\circ}$ direction: Symmetric modes -..... - Antisymmetric modes 


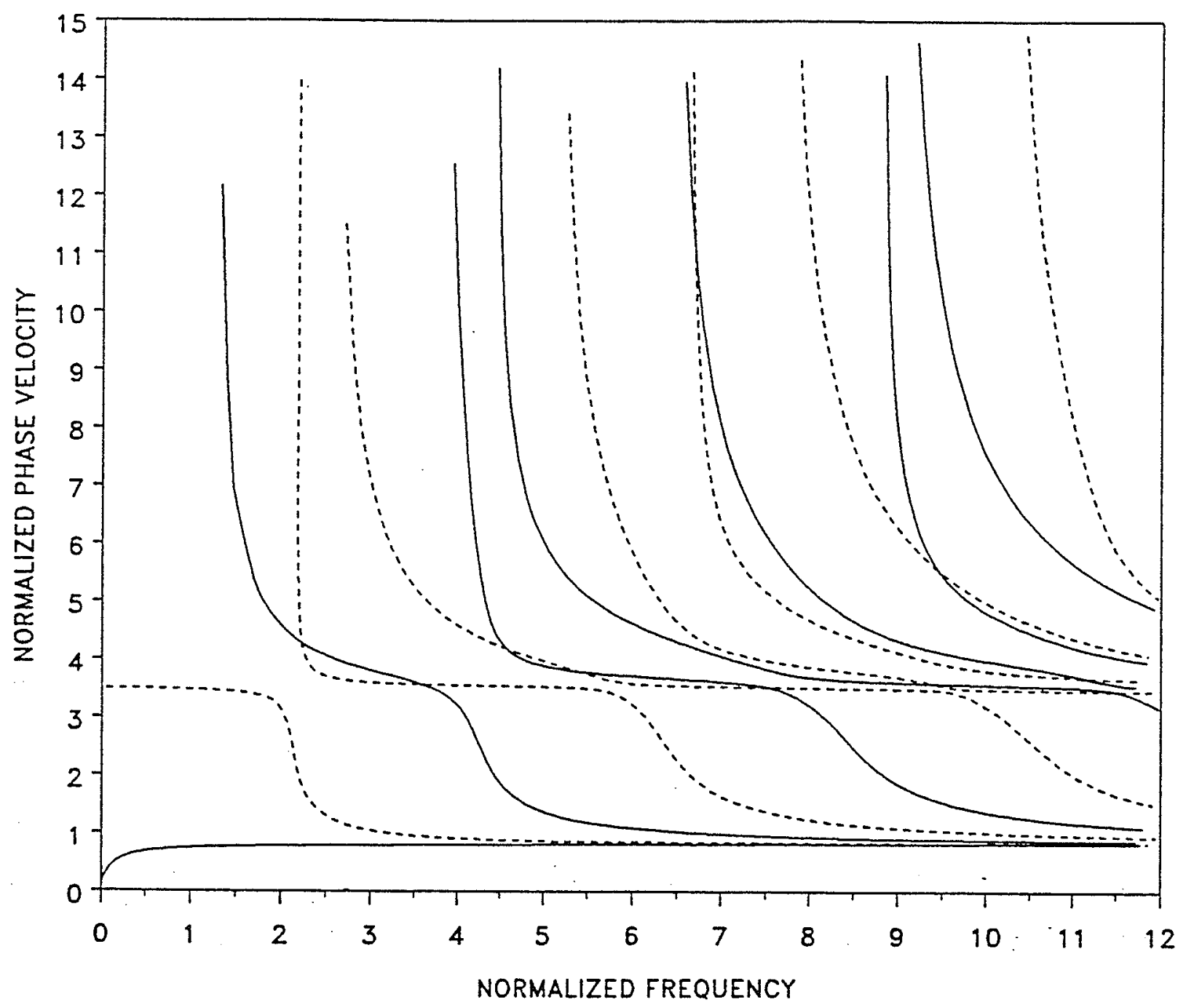

Figure 2.21 : Dispersion of waves in a 35-layer cross-ply $\left(0^{\circ} / 90^{\circ} / 0^{\circ} / \cdots \cdots / 0^{\circ} / 90^{\circ} / 0^{\circ}\right)$ graphite-epoxy plate, for propagation in the $0^{\circ}$ direction:

Symmetric modes -....-; Antisymmetric modes 


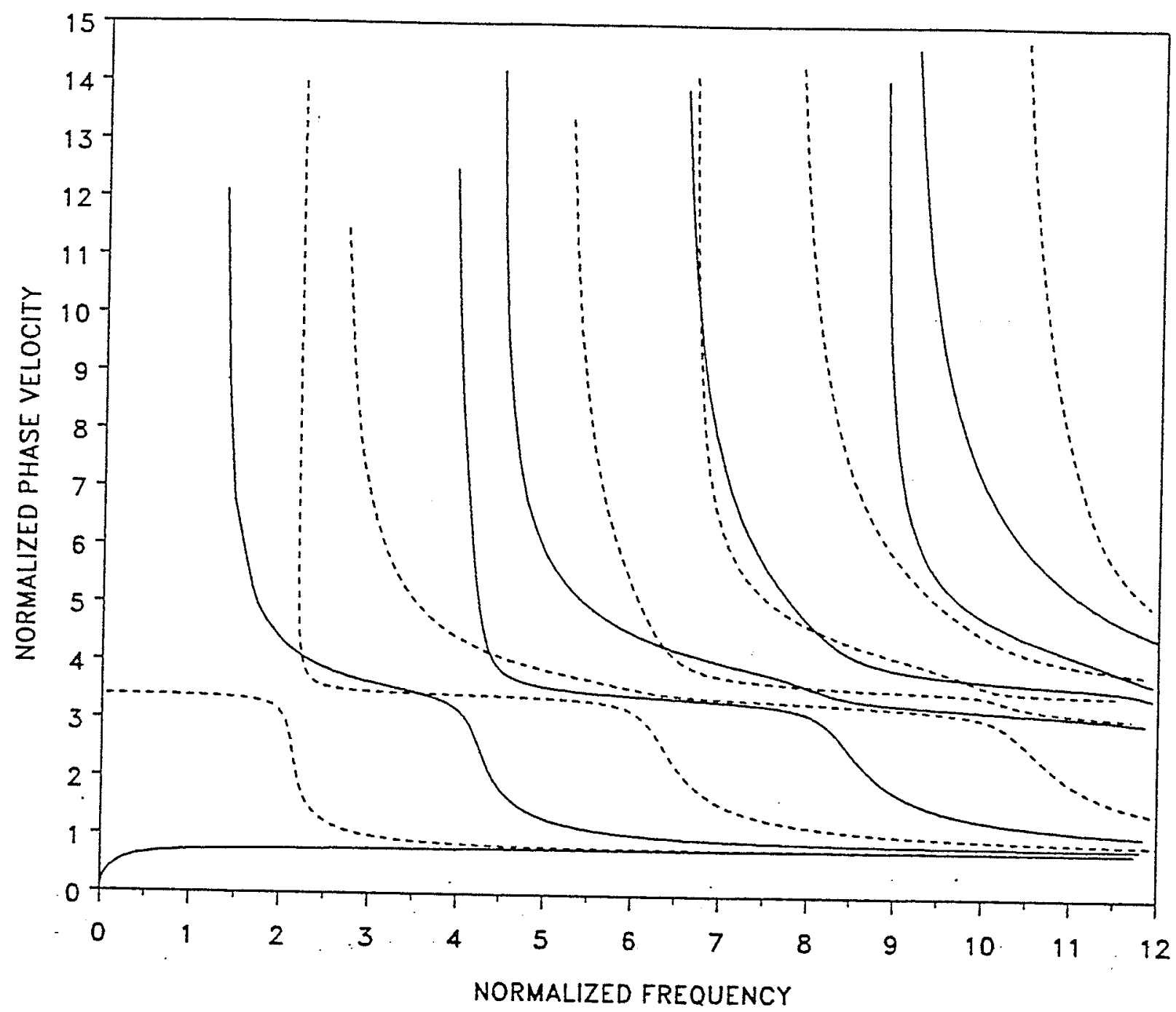

Figure 2.22: Dispersion of waves in a 35-layer cross-ply $\left(0^{\circ} / 90^{\circ} / 0^{\circ} / \ldots \ldots / 0^{\circ} / 90^{\circ} / 0^{\circ}\right)$ graphite-epoxy plate, for propagation in the $90^{\circ}$ direction:

Symmetric modes -...-. - Antisymmetric modes 


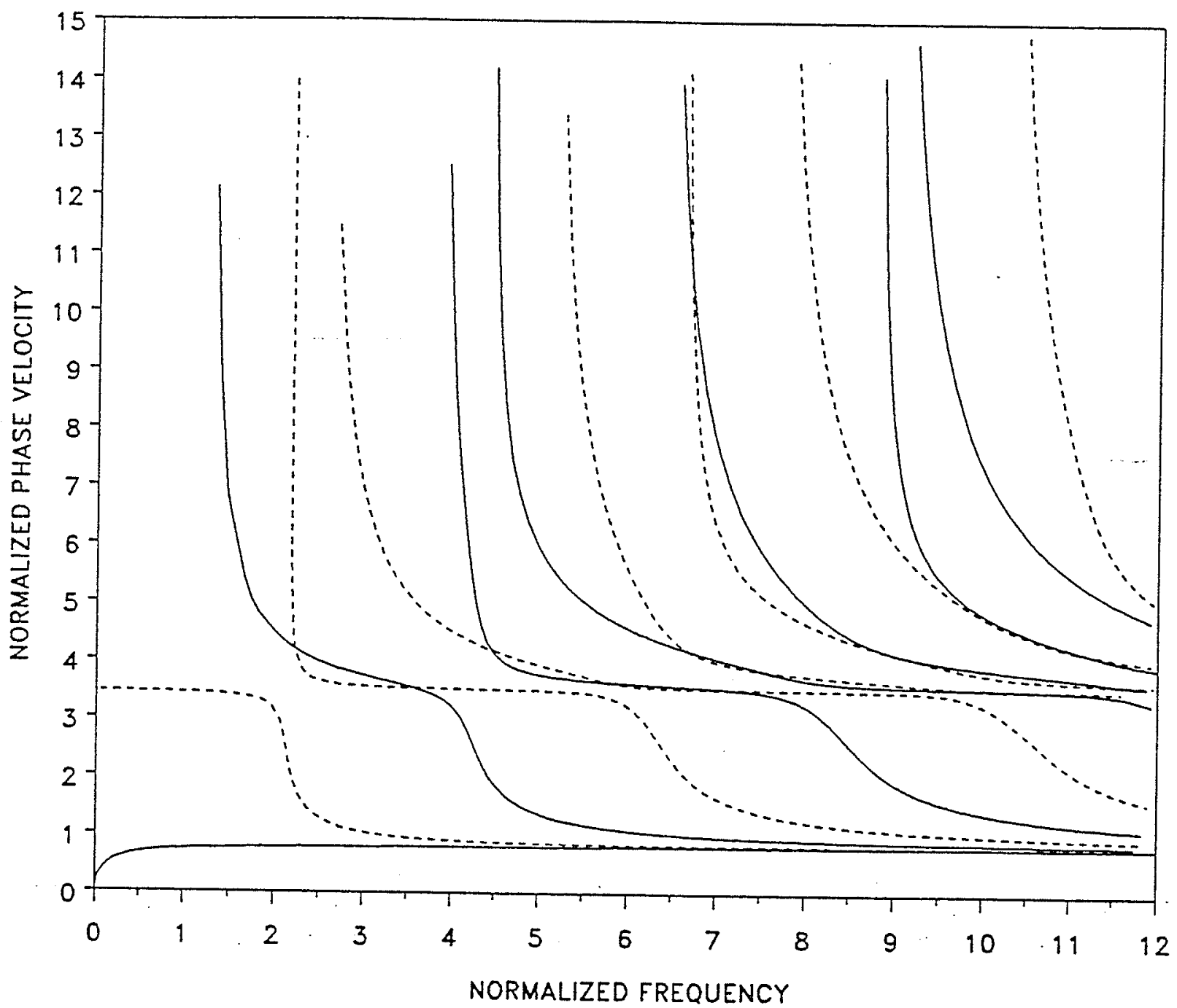

Figure 2.23 : Prediction of dispersion from the effective modulus model, for propagation in the $0^{\circ}\left(90^{\circ}\right)$ direction:

Symmetric modes - . . - . ; Antisymmetric modes —. 


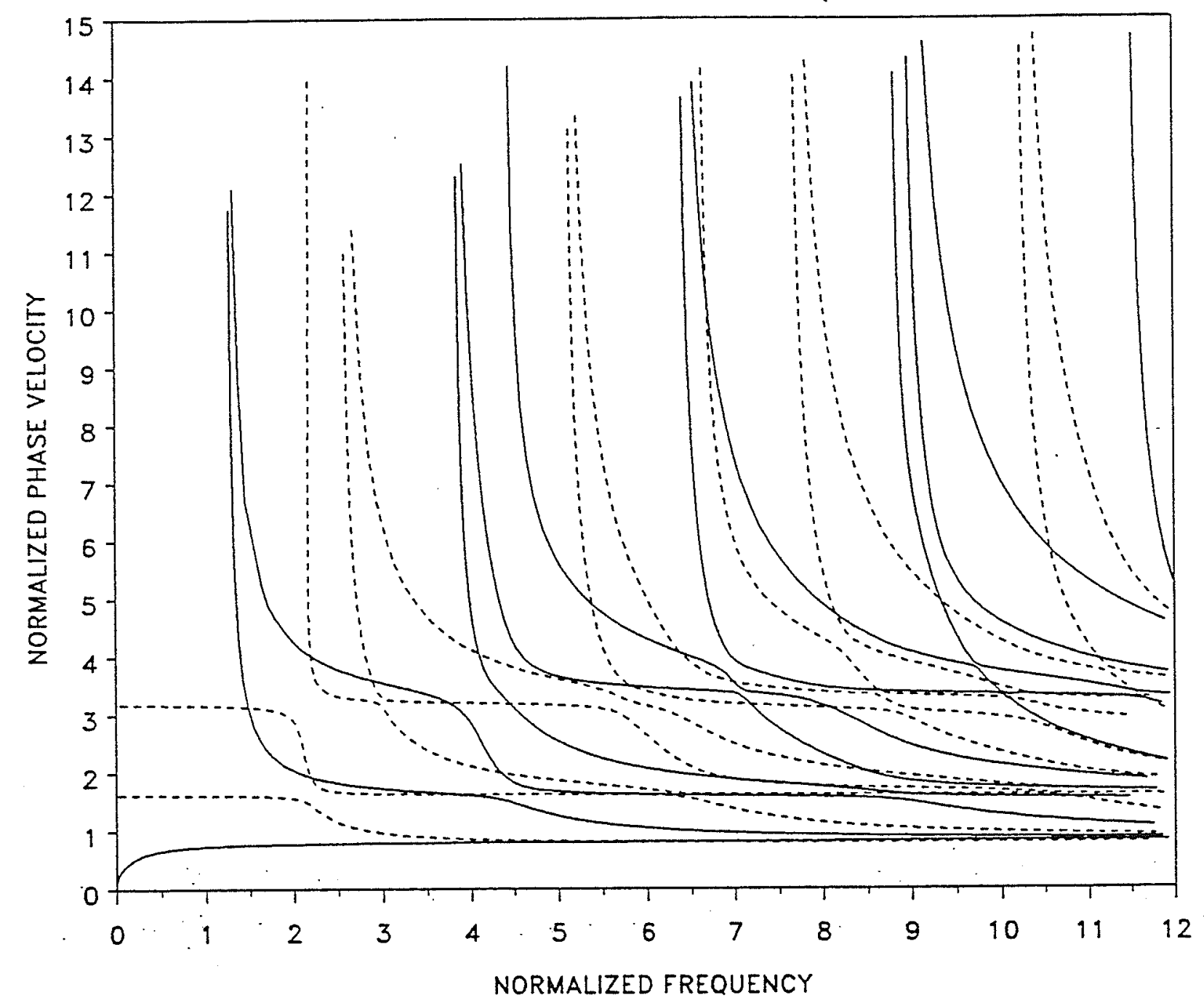

Figure 2.24: Dispersion of waves in a 35-layer cross-ply $\left(0^{\circ} / 90^{\circ} / 0^{\circ} / \cdots \cdots / 0^{\circ} / 90^{\circ} / 0^{\circ}\right)$ graphite-epoxy plate, for propagation in the $22.5^{\circ}$ direction: Symmetric modes -.....; Antisymmetric modes - 


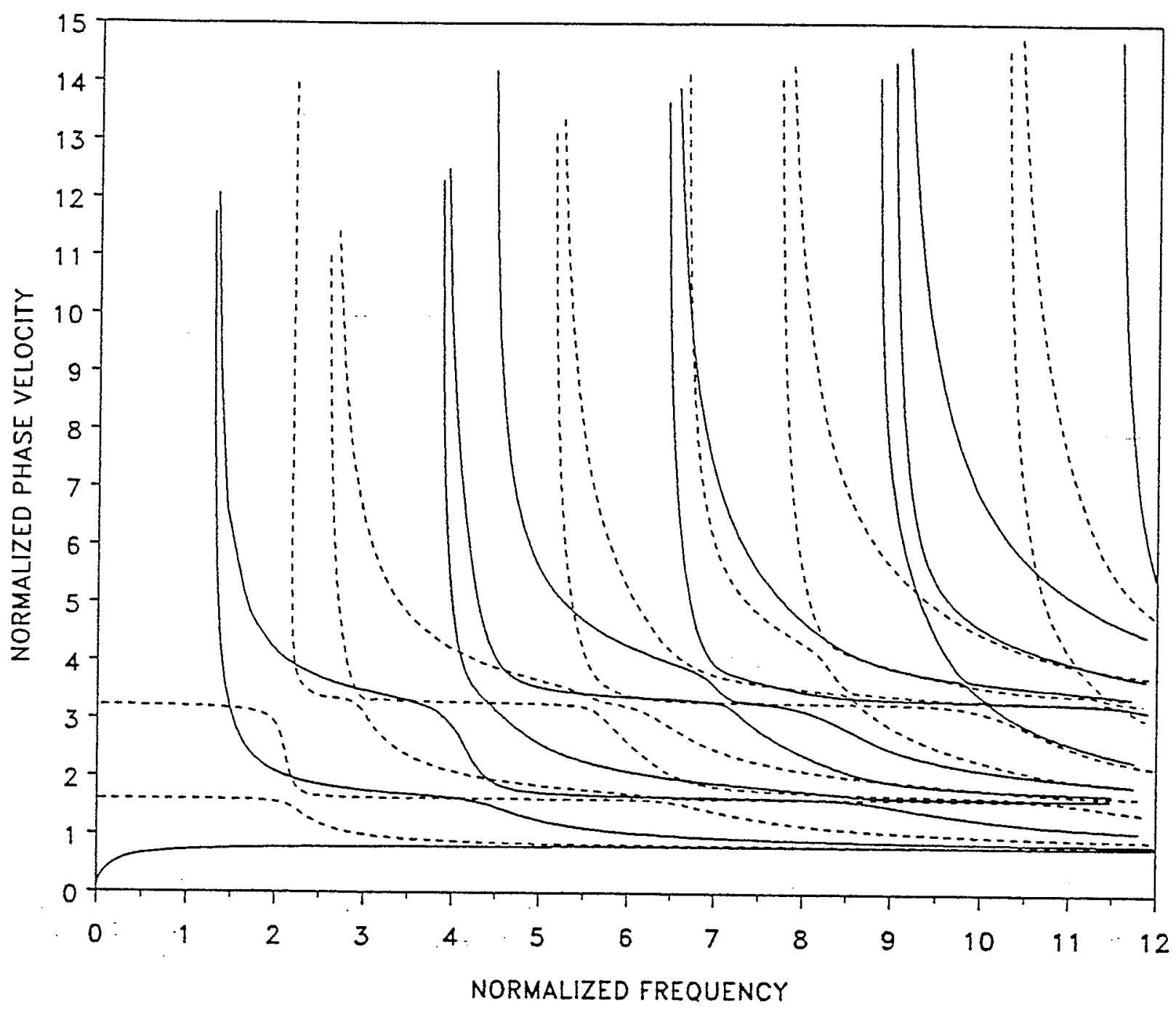

Figure 2.25 : Prediction of dispersion from the effective modulus model, for propagation in the $22.5^{\circ}$ direction:

Symmetric modes - . . . - ; Antisymmetric modes - 


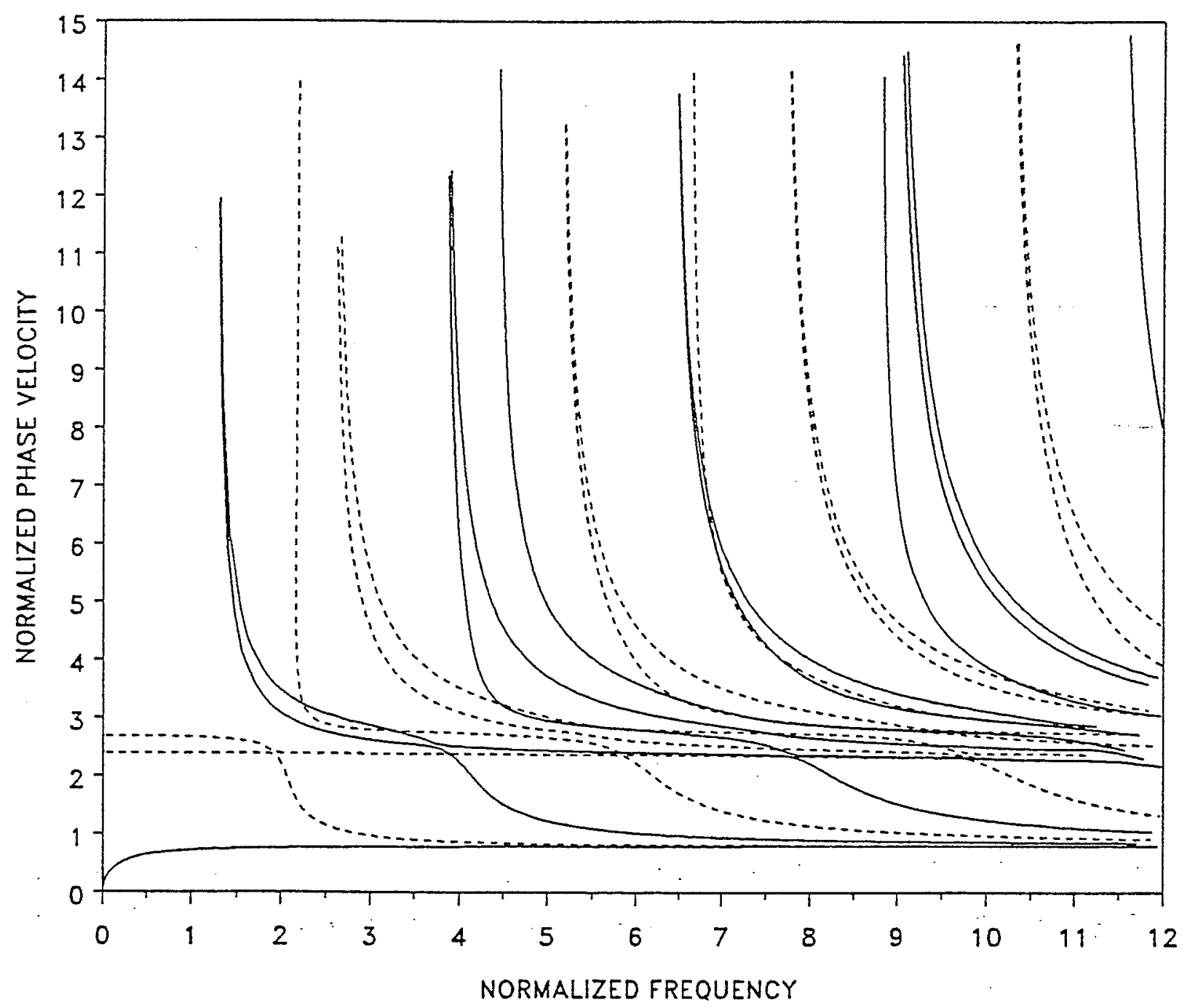

Figure 2.26 : Dispersion of waves in a 35-layer cross-ply $\left(0^{\circ} / 90^{\circ} / 0^{\circ} / \cdots \cdots / 0^{\circ} / 90^{\circ} / 0^{\circ}\right)$ graphite-epoxy plate, for propagation in the $45^{\circ}$ direction: Symmetric modes - ..... - ; Antisymmetric modes - 


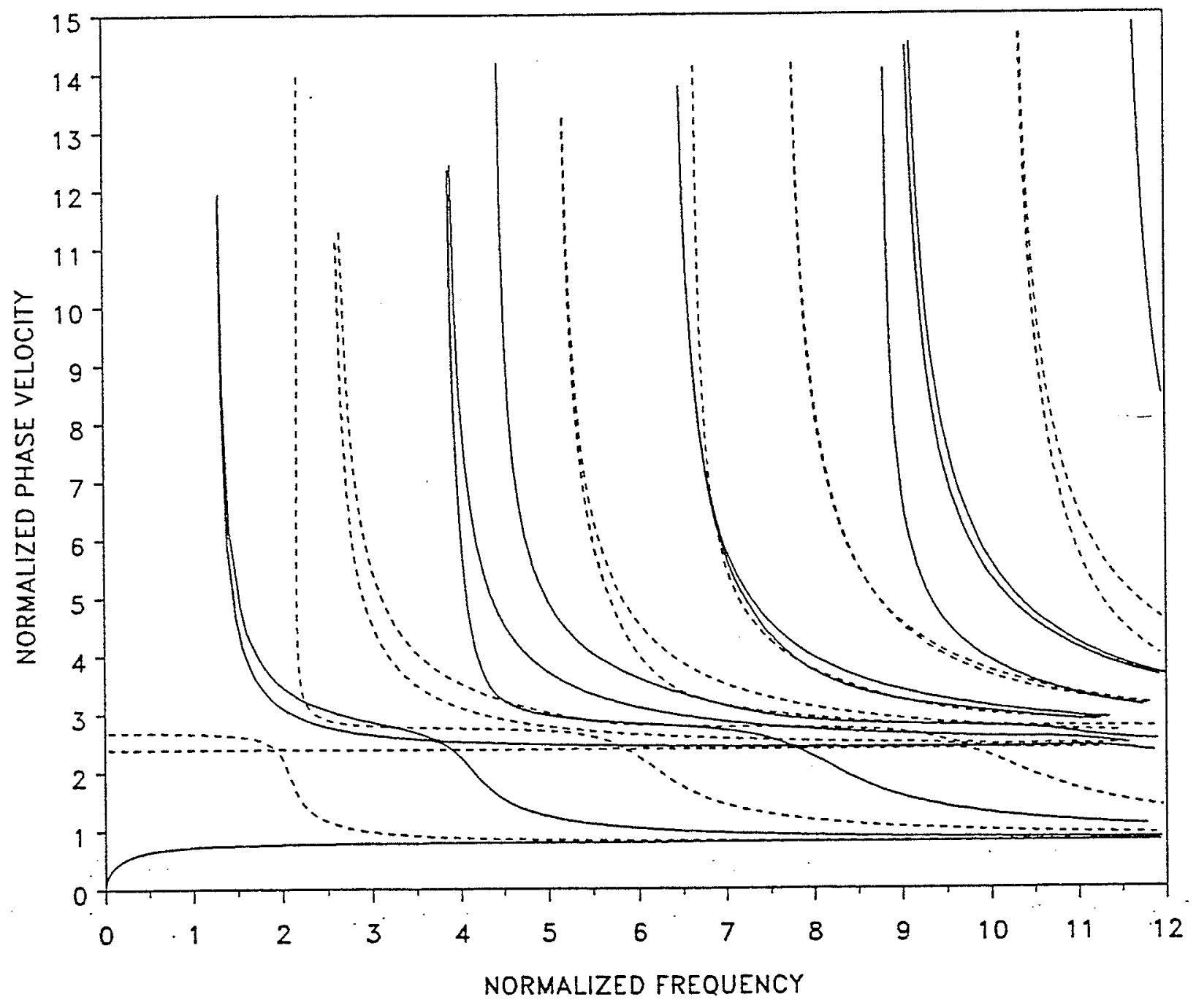

Figure 2.27 : Prediction of dispersion from the effective modulus model, for propagation in the $45^{\circ}$ direction: Symmetric modes - . . . . ; Antisymmetric modes 


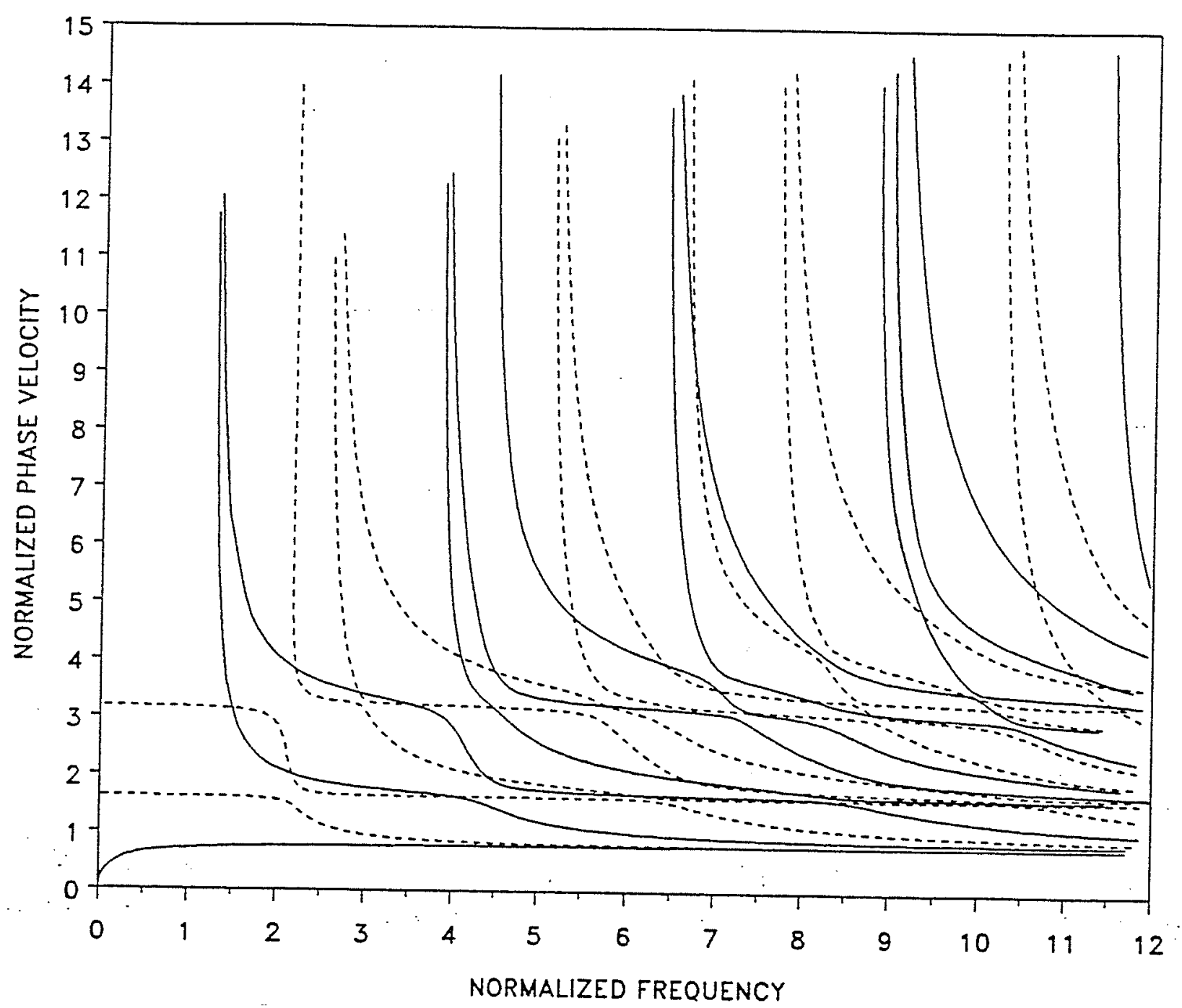

Figure 2.28: Dispersion of waves in a 35-layer cross-ply $\left(0^{\circ} / 90^{\circ} / 0^{\circ} / \cdots \cdots / 0^{\circ} / 90^{\circ} / 0^{\circ}\right)$ graphite-epoxy plate, for propagation in the $67.5^{\circ}$ direction: Symmetric modes - .....; Antisymmetric modes 
Table 2.1

Comparison among results for frequencies from stiffness method I, stiffness method II, and analytical method, for propagation in the $45^{\circ}$ direction in a homogeneous graphite-epoxy plate (real symmetric modes only).

\begin{tabular}{|c|c|r|r|r|r|r|}
\hline \hline Stiffness & & \multicolumn{5}{|c|}{$\Omega$ at $\gamma=\pi$} \\
\cline { 2 - 7 } Method & $\hat{M}$ & $\hat{N}=2$ & $\hat{N}=4$ & $\hat{N}=8$ & $\hat{N}=16$ & Analytical \\
\hline \multirow{6}{*}{ I } & 1 & 2.7896 & 2.7825 & 2.7822 & 2.7822 & 2.7822 \\
& 2 & 3.6370 & 3.6366 & 3.6366 & 3.6366 & 3.6366 \\
& 3 & 4.2288 & 4.2257 & 4.2256 & 4.2256 & 4.2256 \\
& 4 & 5.2572 & 5.2435 & 5.2429 & 5.2429 & 5.2429 \\
& 5 & 7.0209 & 6.9215 & 6.9185 & 6.9184 & 6.9184 \\
& 6 & 7.6411 & 7.4743 & 7.4701 & 7.4699 & 7.4699 \\
& 7 & 10.7008 & 9.2537 & 9.2428 & 9.2417 & 9.2417 \\
\hline \multirow{4}{*}{ II } & 1 & 2.7983 & 2.7847 & 2.7824 & 2.7822 & \\
& 2 & 3.6428 & 3.6371 & 3.6366 & 3.6366 & \\
& 3 & 4.2534 & 4.2280 & 4.2258 & 4.2256 & \\
& 4 & 5.5491 & 5.2598 & 5.2441 & 5.2430 & \\
& 5 & 7.3745 & 6.9792 & 6.9230 & 6.9187 & \\
& 6 & 8.6396 & 7.5375 & 7.4749 & 7.4703 & \\
& 7 & 10.8250 & 10.0289 & 9.2751 & 9.2439 & \\
\hline \hline
\end{tabular}

Note : $\hat{N}=$ Number of sublayers through the half-thickness of the plate $\hat{M}=$ Mode number 
Table 2.2

Comparison among results for frequencies from stiffness method I, stiffness method II, and analytical method, for propagation in the $45^{\circ}$ direction in a 3-layer cross-ply $\left(0^{\circ} / 90^{\circ} / 0^{\circ}\right)$ graphite-epoxy plate

(real symmetric modes only).

\begin{tabular}{|c|c|r|r|r|r|r|}
\hline \hline Stiffness & & \multicolumn{5}{|c|}{$\Omega$ at $\gamma=\pi$} \\
\cline { 3 - 7 } Method & $\hat{M}$ & $\hat{N}=3$ & $\hat{N}=6$ & $\hat{N}=12$ & $\hat{N}=24$ & Analytical \\
\hline & 1 & 2.9338 & 2.9327 & 2.9326 & 2.9326 & 2.9326 \\
& 2 & 3.6295 & 3.6283 & 3.6283 & 3.6283 & 3.6283 \\
& 3 & 4.4421 & 4.4402 & 4.4402 & 4.4402 & 4.4402 \\
& 4 & 5.7145 & 5.7088 & 5.7086 & 5.7086 & 5.7086 \\
& 5 & 7.0667 & 7.0584 & 7.0582 & 7.0582 & 7.0582 \\
& 6 & 8.1816 & 8.1467 & 8.1450 & 8.1450 & 8.1450 \\
& 7 & 8.7594 & 8.7099 & 8.7073 & 8.7072 & 8.7072 \\
& 8 & 10.5329 & 10.4341 & 10.4298 & 10.4297 & 10.4297 \\
& 9 & 10.9441 & 10.8345 & 10.8311 & 10.8309 & 10.8309 \\
& 10 & 11.5937 & 11.4620 & 11.4583 & 11.4581 & 11.4581 \\
\hline \multirow{4}{*}{ II } & 1 & 2.9419 & 2.9336 & 2.9327 & 2.9326 & \\
& 2 & 3.6363 & 3.6291 & 3.6284 & 3.6283 & \\
& 3 & 4.4615 & 4.4419 & 4.4403 & 4.4402 & \\
& 4 & 5.7662 & 5.7134 & 5.7089 & 5.7086 & \\
& 5 & 7.1091 & 7.0638 & 7.0586 & 7.0583 & \\
& 6 & 8.8619 & 8.1802 & 8.1475 & 8.1451 & \\
& 7 & 9.5884 & 8.7498 & 8.7106 & 8.7074 & \\
& 8 & 10.7545 & 10.4947 & 10.4353 & 10.4301 & \\
& 9 & 11.2809 & 10.8839 & 10.8351 & 10.8312 & \\
& 10 & 12.2047 & 11.5234 & 11.4632 & 11.4584 & \\
\hline \hline
\end{tabular}

Note : $\hat{N}=$ Number of sublayers through the half-thickness of the plate $\hat{M}=$ Mode number 


\section{C h a pter 3}

\section{Reflection of Waves at a Free Edge of a Laminated Composite Plate}

\subsection{General}

The present chapter is concerned with the investigation of the free end reflection that occurs when a train of waves travelling in a composite plate strikes a free edge. For simplicity in analysis, attention is confined here only to the timeharmonic wave reflection in plane strain case where the waves are propagating either along or perpendicular to the fibers. However, theoretically any time variation can be considered by using the Fourier Transform Technique. The geometry of the problem considered is depicted in Figure 3.1. When the incident wave strikes the edge $x=0$, a reflected wave field will be generated. The reflected field consists of a finite number of propagating modes and an infinite number of nonpropagating and evanescent modes. A finite number of wave functions are superposed to represent the reflected wave field. Amplitudes of reflected waves are determined by satisfying the traction-free edge condition by the least-squares and variational principle methods. The accuracy of the methods are demonstrated by comparing the results with existing results for a homogeneous isotropic plate and by satisfying energy balance. It is shown that for a laminated composite plate, the least-squares method yields anomalous results. Numerical results from the variational principle method are presented for a homogeneous graphite-epoxy plate and for a 35-layer cross-ply $\left(90^{\circ} / 0^{\circ} / \cdots / 90^{\circ} / 0^{\circ} / 90^{\circ} / \cdots / 0^{\circ} / 90^{\circ}\right)$ laminated graphite-epoxy plate. In each case, the division of energy among various reflected modes is also presented. The end resonance is reported for the homogeneous graphite-epoxy plate. 


\subsection{Description of the Problem}

A time-harmonic plane strain wave excited at $x=+\infty$, propagates in a semiinfinite composite plate in the negative $x$ direction and is incident upon the end $x=0$ (Figure 3.1 ). The plate is composed of perfectly bonded layers with possibly distinct mechanical properties and thickness. Each layer of the composite plate is assumed to have orthotropic material properties. The two faces of the plate $z=0$ and $z=H$, and the edge $x=0$ are traction-free. The incident wave, upon striking the free end, generates a reflected wave field. The objective is to investigate this reflected wave field.

\subsection{Wave Functions}

Wave functions required for the reflection analysis are obtained by considering the plane strain wave propagation in the corresponding infinite plate. For this purpose, each layer is divided into several sublayers so that the total number sublayers through the thickness, $H$, is $N$. The nonvanishing displacement components in the plane strain case are $u(x, z, t)$ and $w(x, z, t)$ in $x$ and $z$ directions, respectively. Following the analysis given in $\$ 2.3 .2$, the dispersion equation governing the plate modes are obtained from equation (2.19) as

$$
f(\omega, k)=P_{31} P_{42}-P_{32} P_{41}=0
$$

For a particular value of $\omega$, equation (3.1) will have a finite number of real roots and an infinite number of imaginary and complex roots for $k$. As reported by Torvik (1967), and Gregory and Gladwell (1983), the admissible $k$ for the reflected wave field of the semi-infinite plate are those real roots with positive group velocity and those non-real roots with $\operatorname{Im}(k)>0$. These conditions ensure that the reflected waves produce bounded displacement and stress fields throughout the plate. It is 
possible to find the roots of equation (3.1) by some search method in a complex wave number plane. However, this approach will be computationally formidable since the roots are sparcely scattered in the complex $k$-plane. As mentioned in chapter 2, Muller's method is employed herein. At the first step, beginning with the highest frequency of interest, the plate is divided into a sufficiently large number of sublayers and the approximate roots are obtained via stiffness method II. To obtain approximate roots, stiffness method II is preferred over stiffness method I mainly due to the fact that, for given $\omega$, stiffness method I involves a fourth order eigenvalue problem whereas stiffness method II involves only a second order eigenvalue problem. One should note here that a fourth order eigenvalue problem involves a much larger computer time and core memory than a second order eigenvalue problem. Those approximate roots lying in the first quadrant of the complex $k$-plane are used as initial guesses in Muller's method to recover the analytical roots. At the next step, $\omega$ is decreased by a small amount and equation (3.1) is solved, taking analytical roots from the previous step as initial guesses for the current step. The process is repeated until the frequency range of interest is scanned. As a check, at some intermediate frequencies, approximate roots from stiffness method II were used as initial guesses in Muller's method to obtain exact roots. After obtaining the wave numbers $k$, for the frequency range of interest, the sign of the real wave numbers were adjusted to have positive group velocities.

To express the reflected wave field as a modal sum to satisfy free edge conditions at $x=0$, all the modes corresponding to the roots with small positive imaginary parts are superposed. Let $M$ be the total number of modes to be used in the modal expansion and $k_{m}$ be the $m$-th root. $M$ number of roots $k$, are ordered as follows: real roots are ordered first in the decreasing order of magnitude. Non-real roots are ordered next in the ascending order of magnitude of their imaginary parts. 
Traction-free conditions at interface 1 and equation (2.16) give the components of the $m$-th eigenvector at interface 1 , as

$$
\left\{B_{1 m}\right\}^{\mathrm{T}}=\left\langle\begin{array}{llll}
1 & -P_{31} / P_{32} & 0 & 0
\end{array}\right\rangle
$$

Then applying equation (2.14) at successive interfaces, the $m$-th mode eigenvector (values of wave function at discrete interfaces) can be obtained, as

$$
\left\{B_{m}\right\}^{\mathrm{T}}=\left\langle\left\{B_{1 m}\right\}^{\mathrm{T}} \quad\left\{B_{2 m}\right\}^{\mathrm{T}} \quad \cdots \quad\left\{B_{i m}\right\}^{\mathrm{T}} \quad \cdots \quad\left\{B_{(N+1) m}\right\}^{\mathrm{T}}\right\rangle
$$

where

$$
\begin{aligned}
& \left\{B_{i m}\right\}^{\mathrm{T}}=\left\langle u_{i m} \quad w_{i m} \quad \sigma_{z z i m} \quad \sigma_{z x i m}\right\rangle, \quad i=1,2, \cdots \cdots, N+1 . \\
& m=1,2, \cdots \cdots, M
\end{aligned}
$$

$u_{i m}, w_{i m}, \sigma_{z z i m}$ and $\sigma_{z x i m}$ are components of the $m$-th mode eigenvector at the $i$-th interface.

If the problem under consideration is symmetric or antisymmtric, it is possible to model only half-thickness of the plate to obtain wave functions, after invoking appropriate boundary conditions at the top and middle surfaces of the plate.

\subsection{Reflection Coefficients}

Consider the case in which the incident wave is the $p$-th propagating mode, corresponding to the wave number $k_{p}$. After striking the edge $x=0$, a reflected wave field will be generated. The displacement vector corresponding to this wave field, $\left\{q_{x}^{\mathrm{r}}\right\}$, at arbitrary $x$, can be approximated by the modal sum of a finite number of modes $M$ in the form

$$
\left\{q_{x}^{\mathrm{r}}\right\}=\sum_{m=1}^{M} A_{m}\left\{q_{m}\right\} \exp \left(j k_{m} x\right) \quad x \geq 0,
$$


where

$$
\begin{aligned}
& A_{m}=\text { amplitude of the } m \text {-th reflected mode, } \\
& \left\{q_{m}\right\}^{\mathrm{T}}=\frac{1}{g_{m}}\left\langle\begin{array}{llllllll}
u_{1 m} & w_{1 m} & \cdots & u_{i m} & w_{i m} & \cdots & u_{(N+1) m} & \left.w_{(N+1) m}\right\rangle,
\end{array}\right. \\
& g_{m}=\sqrt{\sum_{i=1}^{N+1}\left(\left|u_{i m}\right|^{2}+\left|w_{i m}\right|^{2}\right)} .
\end{aligned}
$$

In equation (3.5) and in the subsequent analysis, the time factor $\exp (-j \omega t)$ has been omitted. Equation (3.5) gives the reflected wave field at the edge $x=0$ as

$$
\left\{q_{0}^{\mathrm{r}}\right\}=[G]\{A\},
$$

where

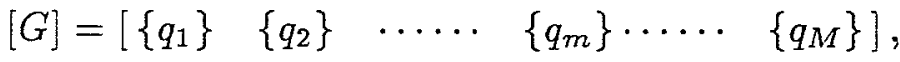

$$
\begin{aligned}
& \{A\}^{\mathrm{T}}=\left\langle\begin{array}{lllll}
A_{1} & A_{2} & \cdots & A_{M}
\end{array}\right\rangle .
\end{aligned}
$$

In view of equations (2.6), (2.7) and (2.9), the $\sigma_{x x}$ component of stresses within the $i$-th sublayer can be expressed as

$$
\sigma_{x x}=\frac{D_{55}}{\Delta_{1}}\left[D_{55}(d e-c f) u+j k(f-B e) \sigma_{z z}\right],
$$

where

$$
\begin{aligned}
& e=\left(1-\delta_{1}\right) A s_{1}^{2}-\lambda_{1} k^{2}, \\
& f=\left(1-\delta_{1}\right) s_{2}^{2}-\lambda_{1} k^{2} B .
\end{aligned}
$$

and $c, d$ and $\Delta_{1}$ are as defined in Appendix B. Since $u_{i m}$ and $\sigma_{z z i m}$ for the $m$ th mode are known from equation (3.3), equation (3.11) can be used to compute 
$\sigma_{x x i m}$ at each interface. It should be noted that $\sigma_{x x}$ is discontinuous at the interfaces between layers. The force vector at the edge due to the reflected field can now be formed as

$$
\left\{R^{\mathrm{r}}\right\}=-[F]\{A\},
$$

where $[F]$ is the force mode shape matrix which represents the nodal force mode shapes at the interfaces, due to stresses $\sigma_{x x}$ and $\sigma_{x z} .[F]$ is a rectangular matrix of size $2(N+1)$ by $M$. In constructing the force vector, the consistent load vector formulation given in Bathe (1982) has been used. The variation of displacements and stresses within the sublayer is assumed to be linear. The explicit form of $[F]$ is given by

$$
[F]=\left[\begin{array}{llllll}
\left\{F_{1}\right\} & \left\{F_{2}\right\} & \cdots \cdots & \left\{F_{m}\right\} & \cdots \cdots & \left\{F_{M}\right\}
\end{array}\right],
$$

where

$$
\begin{aligned}
& \left\{F_{m}^{\mathrm{T}}\right\}=\left\langle\begin{array}{llllllll}
F_{1 m}^{x} & F_{1 m}^{z} & \cdots & F_{i m}^{x} & F_{i m}^{z} & \cdots & F_{(N+1) m}^{x} & F_{(N+1) m}^{z}
\end{array}\right\rangle, \\
& m=1,2, \cdots, M \\
& F_{1 m}^{x}=\frac{h_{1}}{6}\left(2 \sigma_{x x 1 m}^{(+)}+\sigma_{x x 2 m}^{(-)}\right), \\
& F_{1 m}^{z}=\frac{h_{1}}{6}\left(2 \sigma_{z x 1 m}+\sigma_{z x 2 m}\right) \text {, } \\
& F_{i m}^{x}=\frac{h_{i-1}}{6}\left(\sigma_{x x(i-1) m}^{(+)}+2 \sigma_{x x i m}^{(-)}\right)+ \\
& \frac{h_{i}}{6}\left(2 \sigma_{x x i m}^{(+)}+\sigma_{x x(i+1) m}^{(-)}\right) \quad \text { for } \quad 2 \leq i \leq N, \\
& F_{i m}^{z}=\frac{h_{i-1}}{6}\left(\sigma_{z x(i-1) m}+2 \sigma_{z x i m}+\right. \\
& \frac{h_{i}}{6}\left(2 \sigma_{z x i m}+\sigma_{z x(i+1) m}\right) \quad \text { for } \quad 2 \leq i \leq N \text {, } \\
& F_{(N+1) m}^{x}=\frac{h_{N}}{6}\left(\sigma_{x x N m}^{(+)}+2 \sigma_{x x(N+1) m}^{(-)}\right) \text {, } \\
& F_{(N+1) m}^{z}=\frac{h_{N}}{6}\left(\sigma_{z x N m}+2 \sigma_{z x(N+1) m}\right) \text {. }
\end{aligned}
$$


In equations (3.17), $\sigma_{x x i m}^{(-)}$and $\sigma_{x x i m}^{(+)} \quad(1 \leq i \leq N+1)$ denote the $m$-th mode normal stresses in the $x$ direction just, above and below the $i$-th interface, respectively. If the adjacent sublayers of the $i$-th interface have the same material properties, $\sigma_{x x i m}^{(+)}$ will be equal to $\sigma_{x x i m}^{(-)}$.

The edge force vector due to incident field can be written as

$$
\left\{R^{\mathrm{in}}\right\}=A_{p}^{\mathrm{in}}\left\{F_{p}^{-}\right\}
$$

where $A_{p}^{\text {in }}$ is the amplitude of the incident mode and the vector $\left\{F_{p}^{-}\right\}$is obtained from the $p$-th column of $[F]$, after replacing each $x$ direction force component by the negative value of it.

The traction-free edge condition requires that

$$
\{R\}=\left\{R^{\mathrm{r}}\right\}+\left\{R^{\mathrm{in}}\right\}=-[F]\{A\}+A_{p}^{\mathrm{in}}\left\{F_{p}^{-}\right\}=\{0\} .
$$

Subjecting the sum of the squares of the residuals of $\{R\}$ to a least-squares minimization, the least-squares solution for complex amplitudes can be obtained as

$$
\{A\}=A_{p}^{\mathrm{in}}\left[[\bar{F}]^{\mathrm{T}}[F]\right]^{-1}[\bar{F}]^{\mathrm{T}}\left\{F_{p}^{-}\right\} .
$$

A variational solution to the problem can be obtained by applying the principle of virtual displacement as in Wu and Plunkett (1967). This results in

$$
\delta\left\{\bar{q}_{0}\right\}^{\mathrm{T}}\{R\}=\{0\}
$$

where $\delta$ implies first variation, and $\left\{q_{0}\right\}$ denotes the total displacement field at $x=0$. It may be noted that $\left\{q_{0}\right\}$ is given by

$$
\left\{q_{0}\right\}=\left\{q_{0}^{\mathrm{x}}\right\}+\left\{q_{0}^{\text {in }}\right\}
$$


and

$$
\delta\left\{q_{0}\right\}=\delta\left\{q_{0}^{\mathrm{r}}\right\}
$$

where $\left\{q_{0}^{\text {in }}\right\}$ is the displacement vector due to incident field. Substituting equations (3.19) and (3.23) in equation (3.21), and making use of equation (3.8), the variational form of the solution is obtained as

$$
\{A\}=A_{p}^{\text {in }}\left[[\bar{G}]^{\mathrm{T}}[F]\right]^{-1}[\bar{G}]^{\mathrm{T}}\left\{F_{p}^{-}\right\}
$$

Once the amplitudes, $A_{m}$, are known, the displacement and stress field anywhere in the plate can be determined. The reflection coefficient (normalized amplitude) $R_{p m}$ of the $m$-th reflected mode, due to $p$-th incident mode is defined as

$$
R_{p m}=\frac{A_{m}}{A_{p}^{\text {in }}}
$$

\subsection{Energy Flux}

Reflected energy is carried only by the propagating modes. The instantaneous value of the energy flux associated with the $n$-th reflected mode through a plate cross-section (per unit length in $y$ direction) located at any $x \quad(x \geq 0)$, due to $p$-th incident mode is given by

$$
I_{p n}=-\frac{1}{2}\left[\left\{R_{x n}^{\mathrm{r}}\right\}^{\mathrm{T}} \overline{\frac{d}{d t}\left\{q_{x n}^{\mathrm{r}}\right\}}+\overline{\left\{R_{x n}^{\mathrm{r}}\right\}^{\mathrm{T}}} \frac{d}{d t}\left\{q_{x n}^{\mathrm{r}}\right\}\right], \quad 1 \leq n \leq N_{p r}
$$

where

$$
\begin{array}{r}
\left\{q_{x n}^{\mathrm{r}}\right\}=A_{n}\left\{q_{n}\right\} \exp \left[j\left(k_{n} x-\omega t\right)\right] \\
\left\{R_{x n}^{\mathrm{r}}\right\}=A_{n}\left\{F_{n}\right\} \exp \left[j\left(k_{n} x-\omega t\right)\right]
\end{array}
$$


where $\left\{F_{n}\right\}$ is the $n$-th column of matrix $[F]$. In equation (3.26), $\left\{q_{x n}^{r}\right\}$ and $\left\{R_{x n}^{r}\right\}$ represent, respectively, the dispalcement vector and the force vector associated with the $n$-th reflected mode at a plate cross-section located at $x$; and $N_{p r}$ represents the number of propagating modes in the reflected field. Since derivatives with respect to time are appearing in equation (3.26), the time factor $\exp (-j \omega t)$ has not been dropped in equations (3.28) and (3.29). The time-averaged value of the energy flux, $I_{p n}^{\mathrm{r}}$, is obtained by averaging $I_{p n}$ over one cycle. This is given by

$$
I_{p n}^{\mathrm{r}}=\frac{1}{2 \pi / \omega} \int_{0}^{2 \pi / \omega} I_{p n} d t
$$

After carrying out the integration in equation (3.30) explicitly, $I_{p n}^{\mathrm{r}}$ can be written as

$$
I_{p n}^{\mathrm{r}}=-\operatorname{Re}\left[A_{n}\left\{F_{n}\right\}^{\mathrm{T}} \overline{\left[-j \omega A_{n}\left\{q_{n}\right\}\right]}\right], \quad 1 \leq n \leq N_{p r}
$$

where $\operatorname{Re}[$ ] denotes the real part of the complex quantity inside the square bracket. In view of equation (3.25), and after some algebraic simplifications, equation (3.31) can be written as

$$
I_{p n}^{\mathrm{r}}=\omega\left|A_{p}^{\mathrm{in}}\right|^{2}\left|R_{p n}\right|^{2} \vartheta_{n}, \quad 1 \leq n \leq N_{p r},
$$

where

$$
\vartheta_{n}=\operatorname{Im}\left[\left\{F_{n}\right\}^{\mathrm{T}}\left\{\bar{q}_{n}\right\}\right] .
$$

Im[ ] appearing in equation (3.33) denotes the imaginary part of the complex quantity inside the square bracket. It is important to note here that $I_{p n}^{\mathrm{r}}$ is independent of $x$. Following a similar approach, the time-averaged value of the energy flux of the incident wave can be written as

$$
I_{p}^{\text {in }}=\omega\left|A_{p}^{\text {in }}\right|^{2} \vartheta_{p}
$$


Let $E_{p n}$ be the proportion of incident energy transfered into the $n$-th reflected mode. Then

$$
E_{p n}=\frac{I_{p n}^{\mathrm{r}}}{I_{p}^{\mathrm{in}}} .
$$

Another useful index is the percentage error in energy balance, $\varepsilon$, defined by

$$
\varepsilon=\frac{100}{I_{p}^{\mathrm{in}}}\left[I_{p}^{\mathrm{in}}-\sum_{n=1}^{N_{\mathrm{pr}}} I_{p n}^{\mathrm{r}}\right] .
$$

The principle of energy conservation requires that sum of $E_{p n} \quad\left(n=1,2, \cdots, N_{p r}\right)$ be unity, namely $\varepsilon$ should be zero. This condition is used to assess the accuracy of the analysis presented here.

\subsection{Numerical Results and Discussion}

In this section, the numerical results of the reflection problem for following four examples are presented.

Example 1 - a homogeneous isotropic plate with Poisson's ratio, $\nu=0.25$. The incident wave considered is the first symmetric propagating mode.

Example 2 - a homogeneous graphite-epoxy (transversely isotropic) plate with fibers aligned along the $x$ - axis $\left(0^{\circ}\right)$. See Table 3.1 for material properties. The incident wave is the first symmetric propagating mode.

Example 3 - a 35-layer graphite-epoxy cross-ply laminated plate with $90^{\circ} / 0^{\circ} / \cdots / 90^{\circ} / 0^{\circ} / 90^{\circ} / \cdots / 0^{\circ} / 90^{\circ}$ configuration. Material properties are given in Table 3.1. The incident wave is the first symmetric propagating mode.

Example 4 - same as example 3 , but the incident wave is the first antisymmetric 
propagating mode.

Since in each of the above examples, the problem is either symmetric or antisymmetric, only half-thickness of the plate was considered in the analysis. In all four examples, the real branches of the frequency spectrum were plotted to identify the positive group velocity zones. Figure 3.2 show the frequency spectrum for examples 2-4. The following nondimensionalization is used for frequency and wave number throughout this section:

$$
\begin{aligned}
& \text { Example 1 } \quad \Omega=\frac{\omega H}{2 c_{d} \sqrt{\mu / \rho}}, \gamma=\frac{1}{2} k H, \\
& \text { where } \quad c_{d}=\sqrt{\frac{3 \mu}{\rho}} ; \mu=\text { shear modulus. } \\
& \text { Example 2-4 - } \quad \Omega=\frac{\omega H}{2 \sqrt{\left(D_{55} / \rho\right)_{0^{\circ}}}} \quad, \quad \gamma=\frac{1}{2} k H .
\end{aligned}
$$

The total number of sublayers, $N$, used to compute the eigenvectors and the number of modes, $M$, used in the modal expansion, play an important role in the accuracy of the analysis. In order to select a suitable value for $N$, the quantity $\vartheta_{n}$ $\left(n=1,2, \cdots, N_{\mathrm{pr}}\right)$ defined in equation (3.33) was computed by increasing the value of $N$ at a few selected lower, intermediate and higher frequencies in the frequency range of interest, until converged values were obtained for $\vartheta_{n}$. In this way, $N$ through the half-thickness was chosen as 50 in examples 1 and 2 , and 70 in examples 3 and 4 . Thereafter, the reflection problem was solved at the selected frequencies by the least-squares method [equation (3.20)] and by the variational method [equation (3.24)], by increasing the number of modes. Tables 3.2 and 3.3 show some of the results obtained from two methods for percentage error in energy balance, $\varepsilon$, and the modulus of the reflection coefficient of the first reflected mode, $\left|R_{11}\right|$. In example 1 at $\Omega=4.0$, the reflected field consists of 4 symmetric propagating modes; in example 2 at $\Omega=4.0$, the reflected field consists of three symmetric propagating 
modes; in example 3 at $\Omega=5.1$, the reflected field consists of three symmetric propagating modes; and in example 4 at $\Omega=4.0$, the reflected field consists of three antisymmetric propagating modes. Comparison of the results from the two methods shows that the variational method gives very good energy balance and convergence of the reflection coeffcient $\left|R_{11}\right|$, even with a relatively smaller number of modes. It can be noticed that for the homogeneous plate, there is no noticeable difference in the results from the two methods if a sufficiently large number of modes are taken. For the laminated plate, the results obtained by the least-squares method are alarming. Even with thirty modes participation, only $50 \%$ of the incident mode energy is reflected back into the plate from the free edge for symmetric incidence, which is an anomaly. The reason for this anomaly is obvious. Unlike the variational method which minimizes the energy, the least-squares method does not have a physical basis. In the least-squares method, even though the sum of the squares of the residuals in $\{R\}$ is minimized, the minimized residual sum could be large resulting in large errors in $\varepsilon$. In what follows, only the results obtained from the variational method are presented.

Figure 3.3 shows the comparison of proportion of energy $E_{1 n}$ obtained by the present method with those of Gregory and Gladwell (1983) for an isotropic plate. The modal expansion consisted of 21 modes. For the range of $\Omega$ in Figure 3.3, $|\varepsilon|<0.18 \%$; it is seen that the comparison is excellent. Even though the results for reflection coefficient $\left|R_{11}\right|$ are not presented here, results were in complete agreement with those of Gregory and Gladwell (1983). A full discussion on the energy distribution among reflected modes for this case can be found in Gregory and Gladwell (1983).

The energy distribution among various reflected propagating modes in example 2 is shown in Figure 3.4. The modal expansion consisted of 21 modes. For the range 
$0<\Omega<2.196$ (which is not shown in Figure 3.4), $|\varepsilon|$ was less than $0.05 \%$. For the range of results presented here, $|\varepsilon|<0.88 \%$. The range $2.197<\Omega \leq 2.2041$ is the backward-wave transmisson region discussed by Meitzler (1965), where the third propagating mode has a negative phase velocity. In particular, it was observed that at the first cut-off frequency $\Omega=2.2041$, only the second mode carries energy; at the second cut-off frequency $\Omega=3.142$, only the first mode carries energy; at the third cut-off frequency $\Omega=6.283$, all three modes carry energy and in the range $2.4<\Omega<5.9$, the first and third modes share almost the entire reflected energy. In Figure 3.5(a), the variation of reflection coefficient $\left|R_{11}\right|$ with $\Omega$ is shown. It can be seen that $\left|R_{11}\right|=1.0$ in the range $0<\Omega<2.197$. Since only one propagating mode exists in this frequency range, the entire energy is reflected into the first mode, and therefore, by the energy conservation principle, $\left|R_{11}\right|$ has to be equal to unity. For $\Omega>2.197,\left|R_{11}\right|$ is oscillatory. After a careful search, it was noted that edge resonance occurs in the second mode near $\Omega=2.1520$. The variation of reflection coefficient $\left|R_{12}\right|$ near resonant frequency is shown in Figure 3.5(b). At $\Omega=2.1520$, by increasing $M$ from 20 to $30, \varepsilon$ changed from $0.14 \%$ to $0.01 \%$ and only a $0.11 \%$ increase in $\left|R_{12}\right|$ was observed.

The division of energy between various reflected modes for examples 3 and 4 are presented in Figures 3.6 and 3.7, respectively. Figures 3.8(a) and 3.8(b) show reflection coefficient $\left|R_{11}\right|$, for examples 3 and 4 respectively. The first three cut-off frequencies are $\Omega=2.204,2.556$ and 5.111 for symmetric modes, and $\Omega=1.278$, 3.834 and 4.408 for antisymmetric modes. The symmetric case consisted of 22 modes whilst in the antisymmetric case, 21 modes were used. In the frequency ranges considered, $|\varepsilon|<0.44 \%$ for the symmetric case and $|\varepsilon|<0.18 \%$ for the antisymmetric case. In particular, it is seen from Figures 3.6 and 3.7 that between the second and third cut-off frequencies, in the symmetric case, energy is shared 
almost entirely between first and third modes, whereas in the antisymmetric case, energy is shared among all three modes. A careful search was made for the end resonant frequency in the symmetric case but none could be found.

\subsection{Concluding Remarks}

A semi-analytical method employing exact discrete eigenvectors for displacements and stresses has been used to study the guided plane strain wave reflection at the free edge of a laminated composite plate. Problems were solved by the least-squares method and the variational method. It is found that the variational principle method gives very good results. It is shown that the results agree well with known solutions for homogeneous isotropic plate. Since the exact eigenvectors are employed, the method is accurate at both low frequencies and high frequencies. Since the least-squares method gives anomalous results from the point of view of energy balance, it is concluded that the least-squares method should be used with caution for the free end reflection problem of layered anisotropic plates. Although the case of wave propagation along a principle direction has been studied, the method can be easily applied to off-axis propagation. 


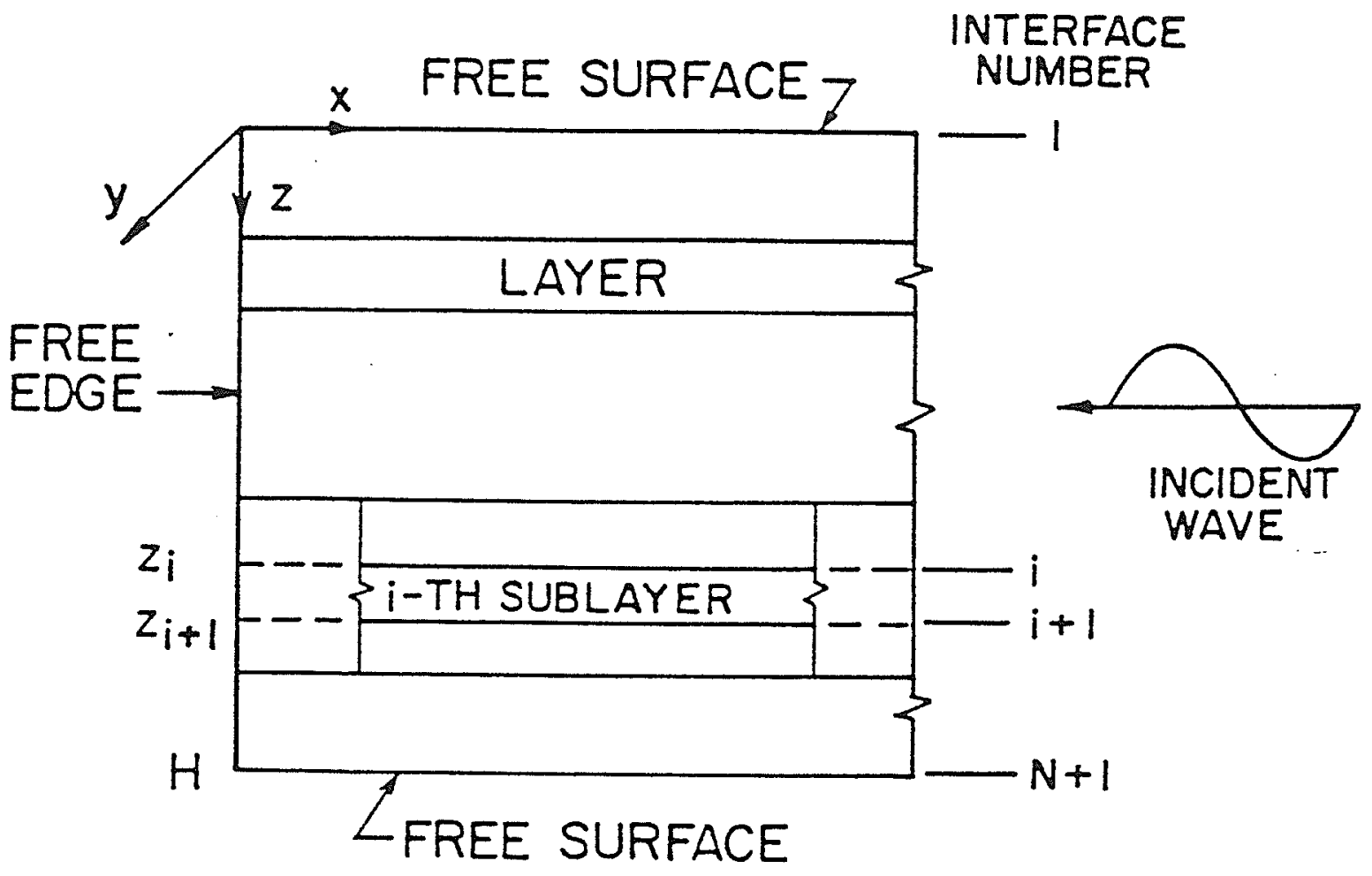

Figure 3.1 : Geometry of the semi-infinite layered plate. 


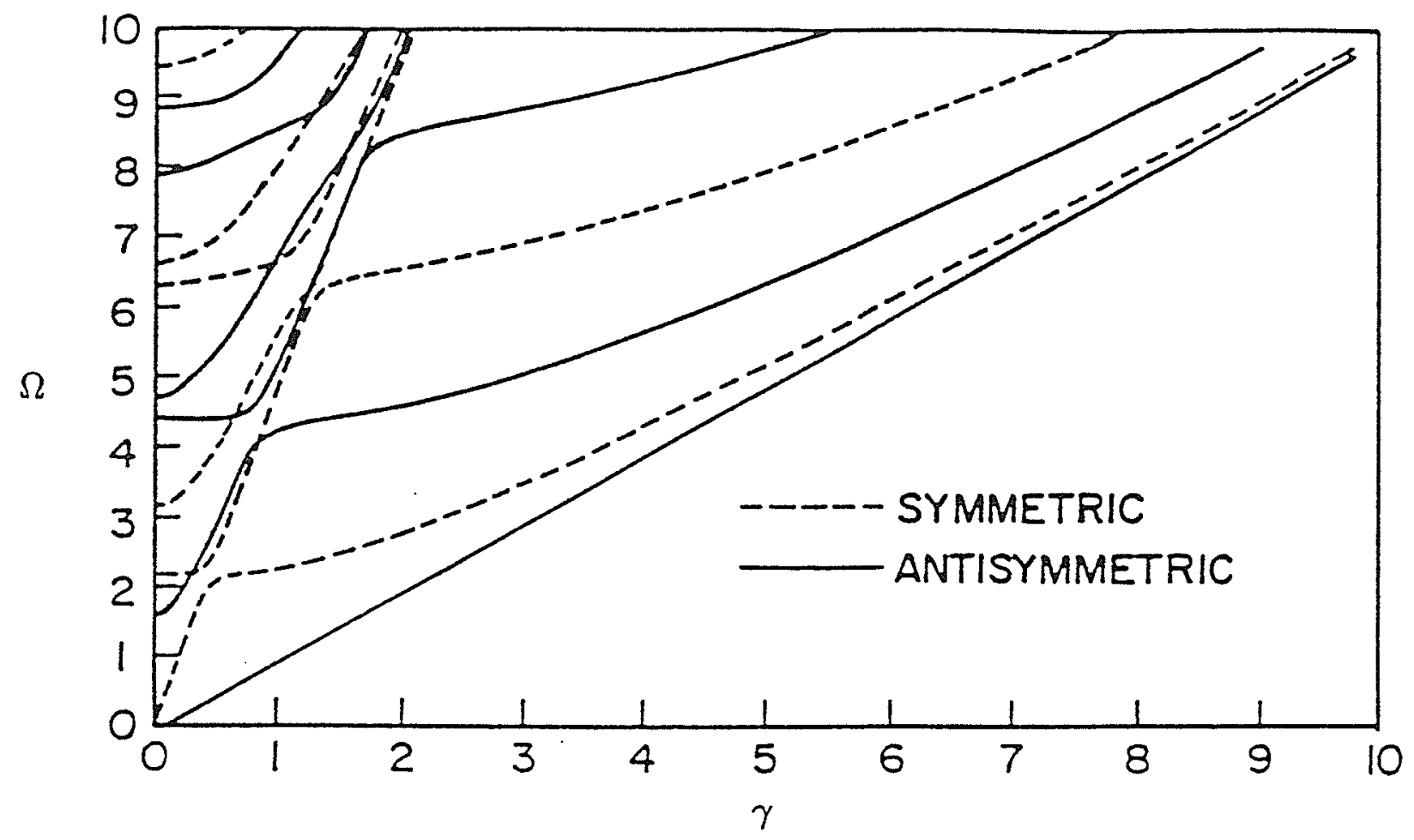

(a)

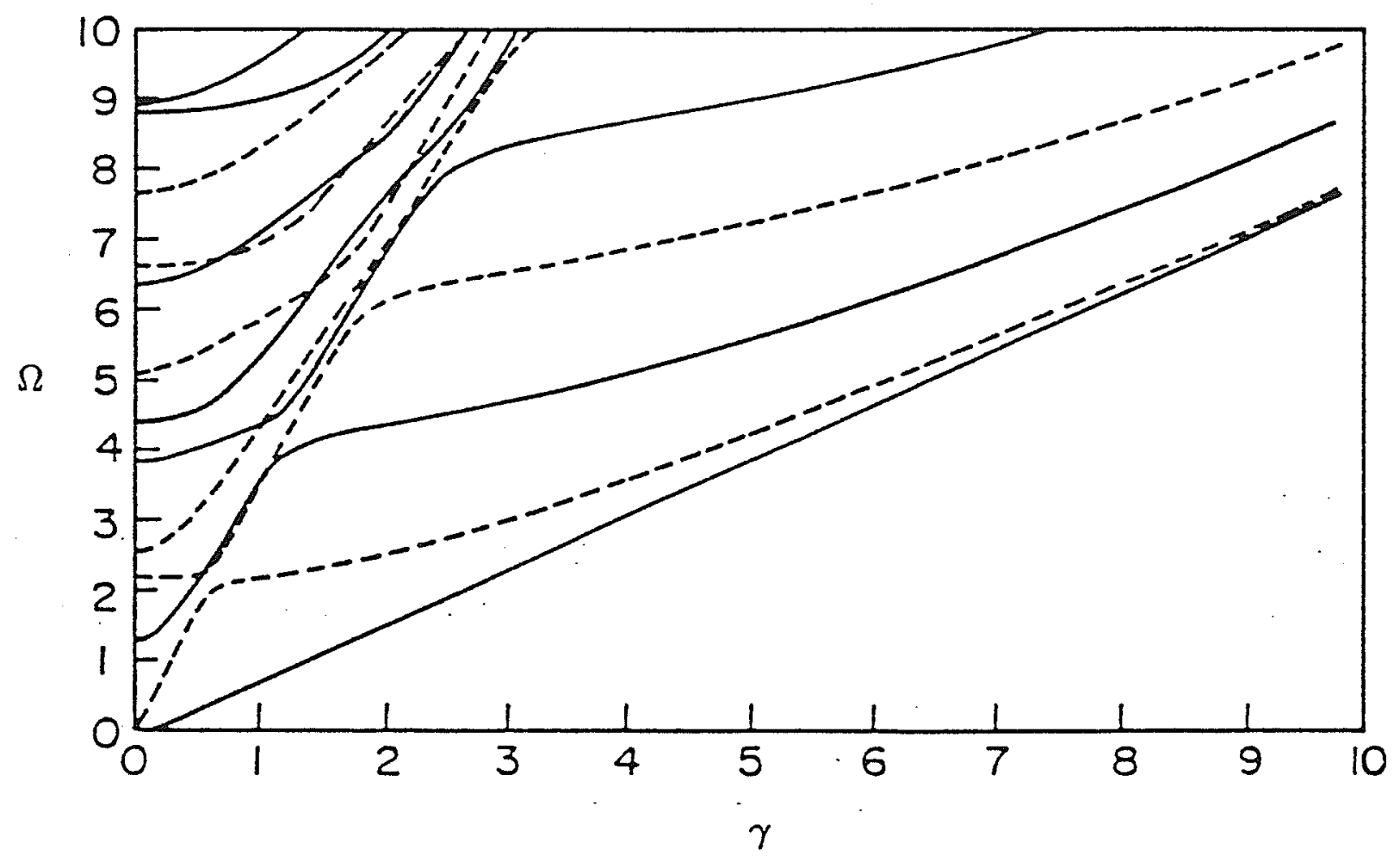

(b)

Figure 3.2 : Real branches of the frequency spectrum

(a) Homogeneous graphite-epoxy plate,

(b) 35-layer cross-ply $\left(90^{\circ} / 0^{\circ} / \cdots / 90^{\circ} / 0^{\circ} / 90^{\circ} / \cdots / 0^{\circ} / 90^{\circ}\right)$ graphite-epoxy plate. 

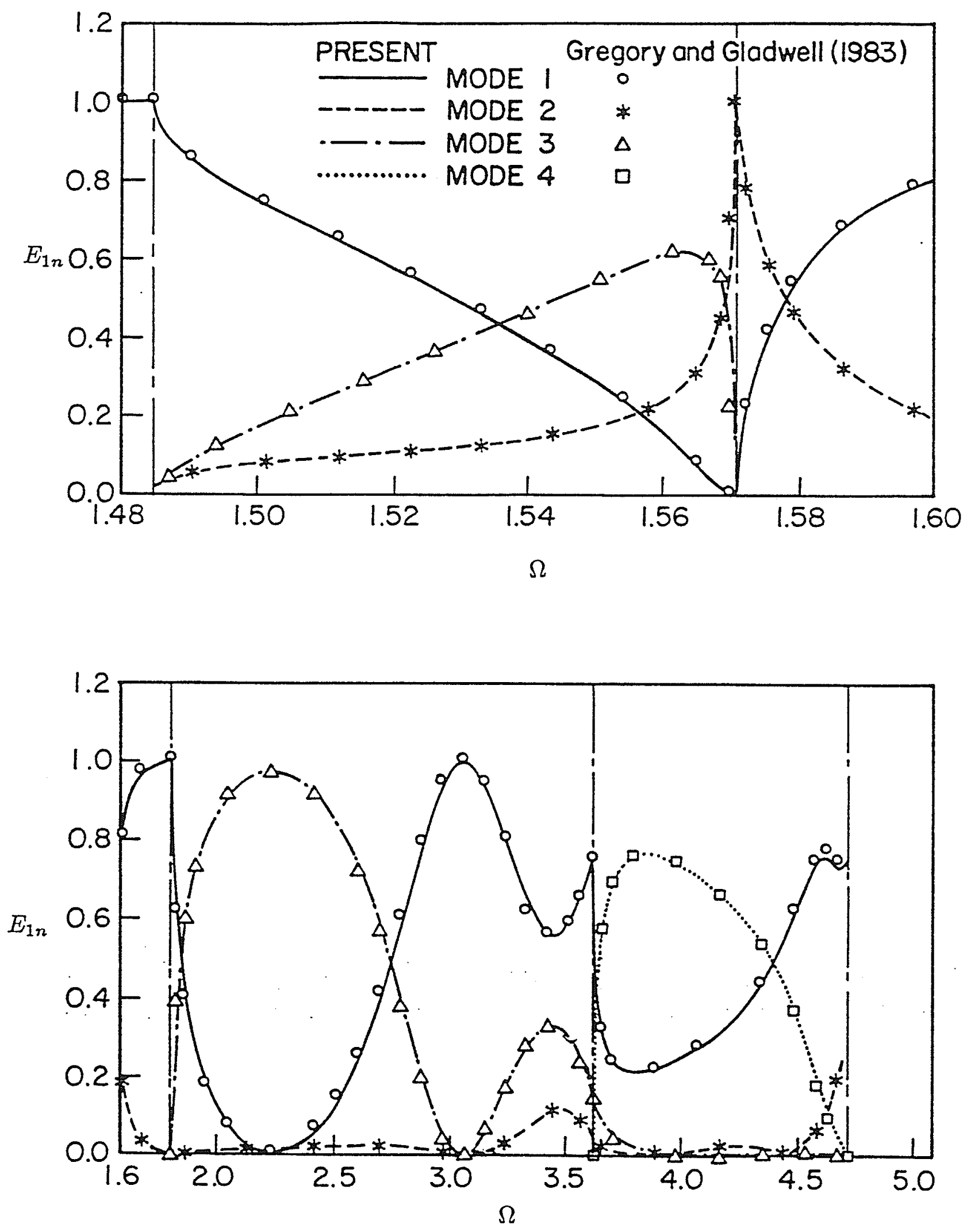

Figure 3.3 : The proportions of energy $E_{1 n}$ vs $\Omega$, for the homogeneous isotropic plate, due to first symmetric incident mode $(\nu=0.25)$. 


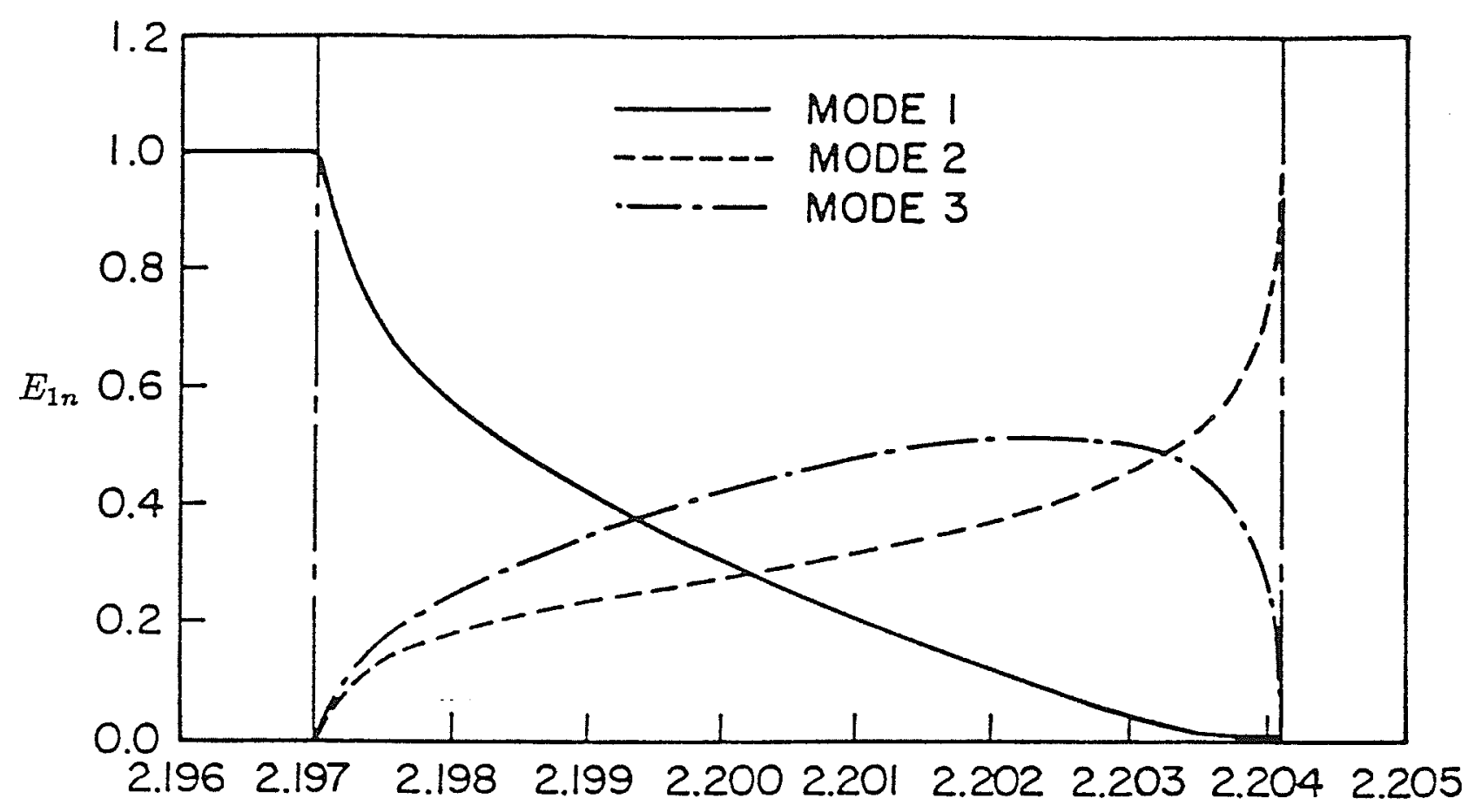

$\Omega$

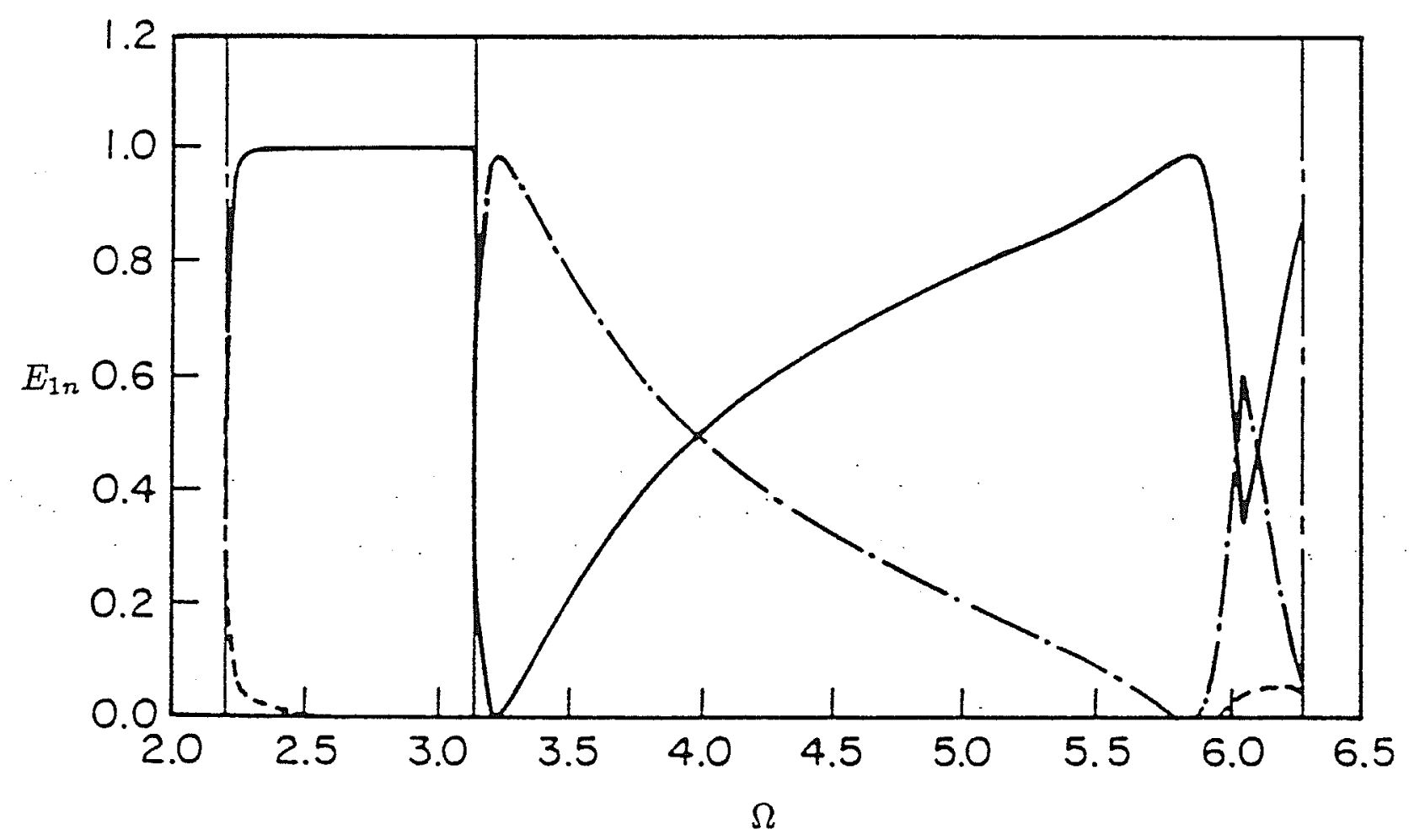

Figure 3.4: The proportions of energy $E_{1 n}$ vs $\Omega$, for the homogeneous graphite-epoxy plate, due to first symmetric incident mode. 


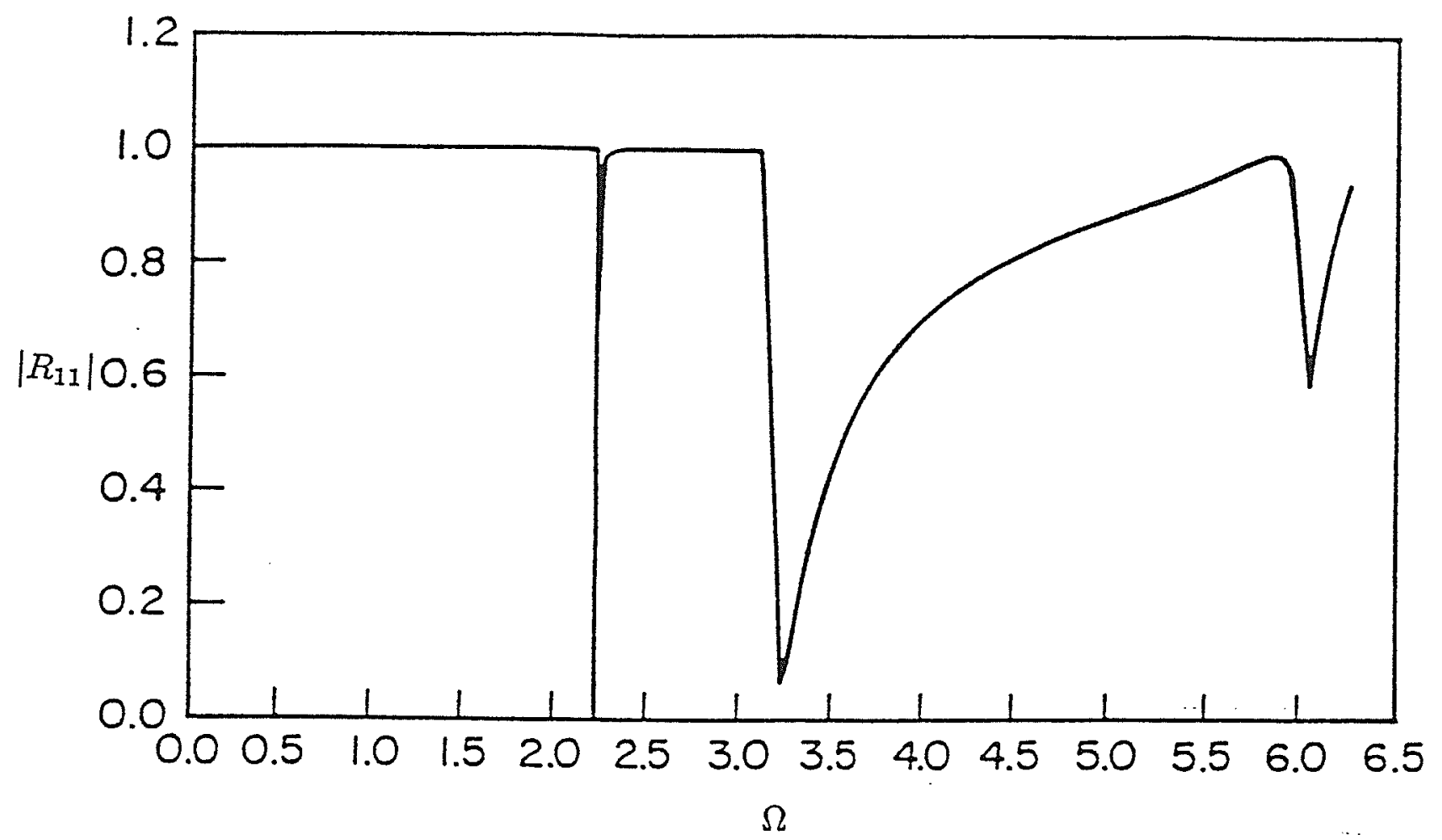

(a):

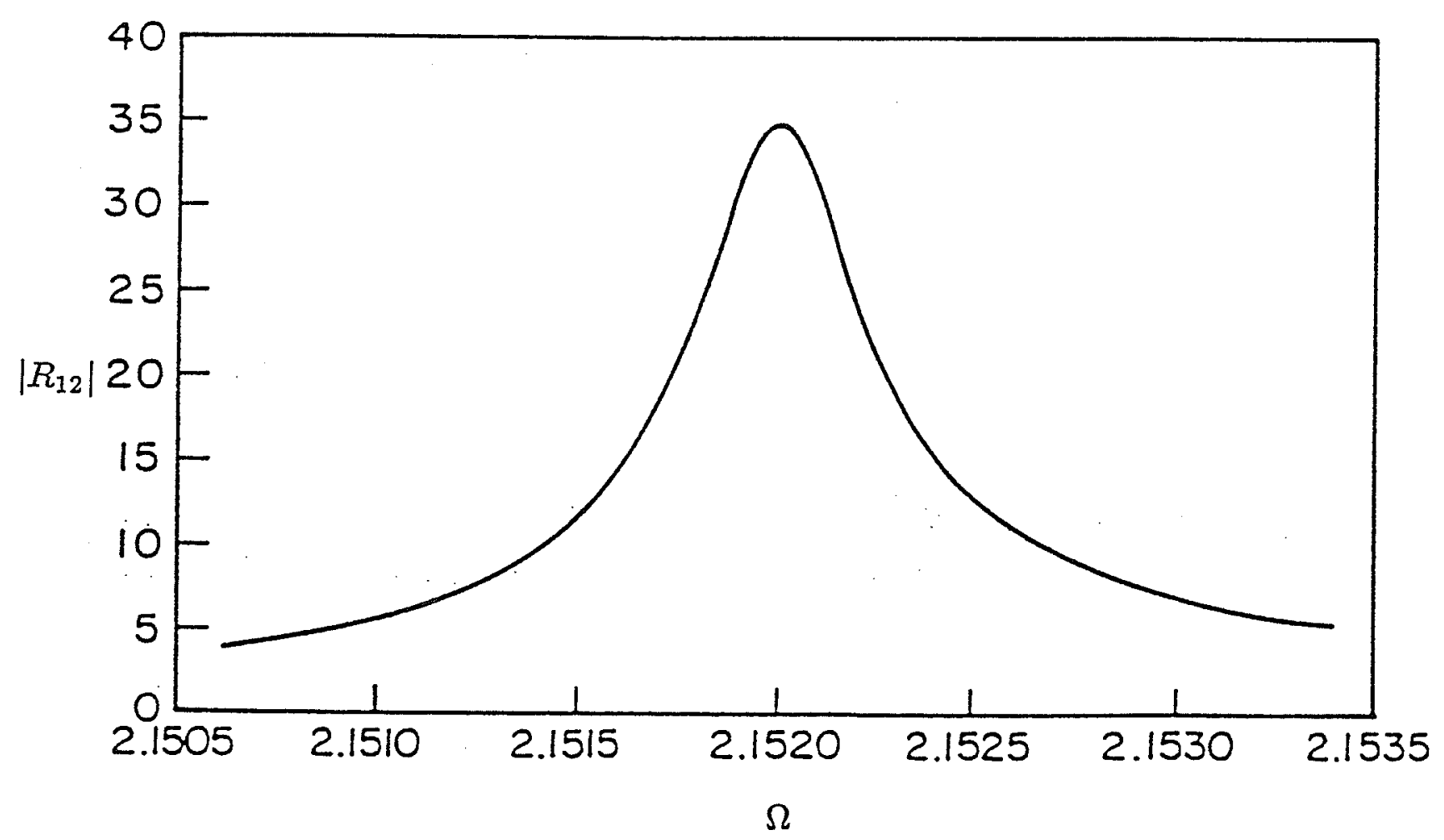

(b)

Figure 3.5: The reflection coefficient $\left|R_{1 n}\right|$ vs $\Omega$, for the homogeneous graphite-epoxy plate, due to first symmetric incident mode

$\begin{array}{ll}\text { (a) } n=1, & \text { (b) } n=2 \text {. }\end{array}$ 

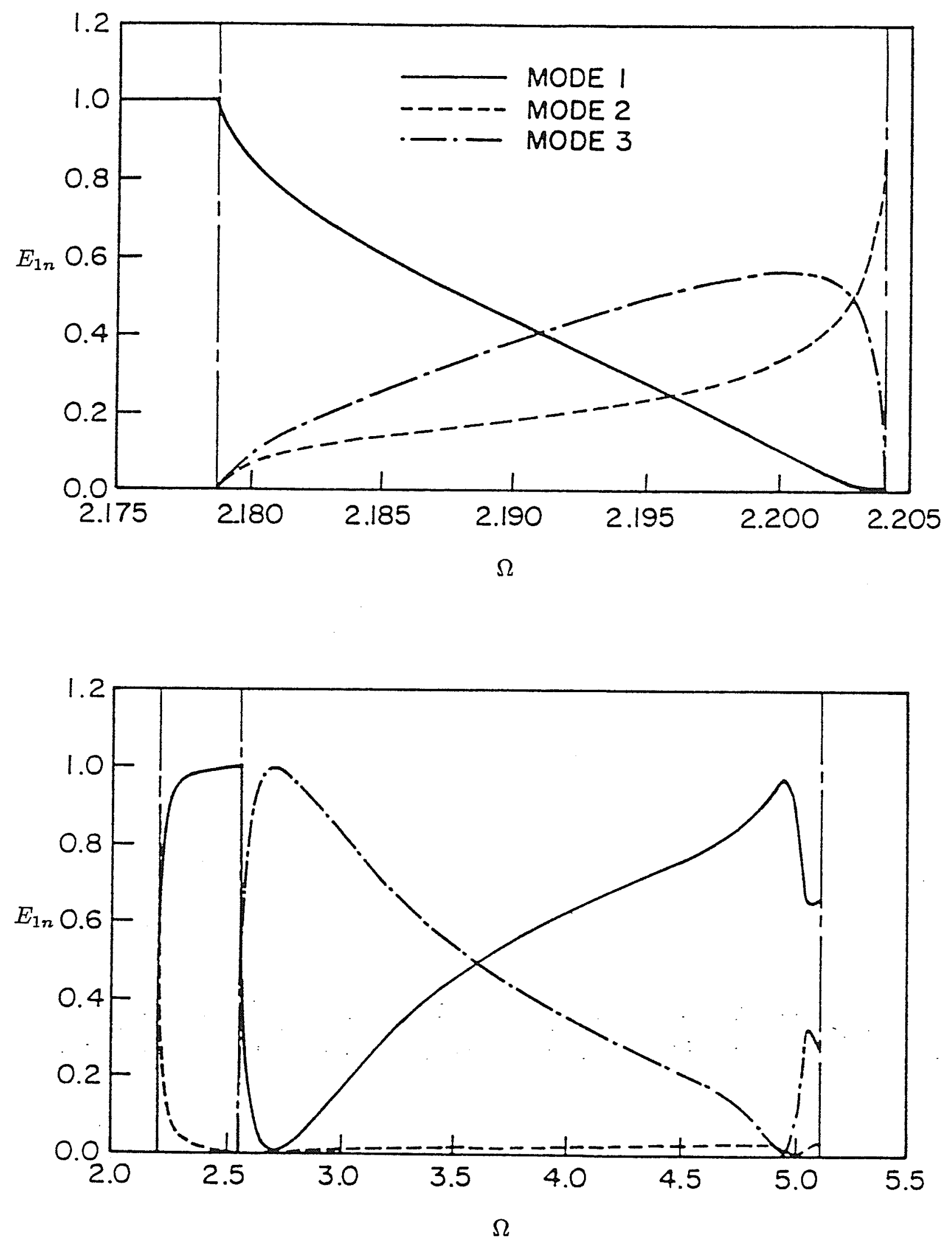

Figure 3.6 : The proportions of energy $E_{1 n}$ vs $\Omega$, for the 35-layer graphite-epoxy plate, due to first symmetric incident mode. 


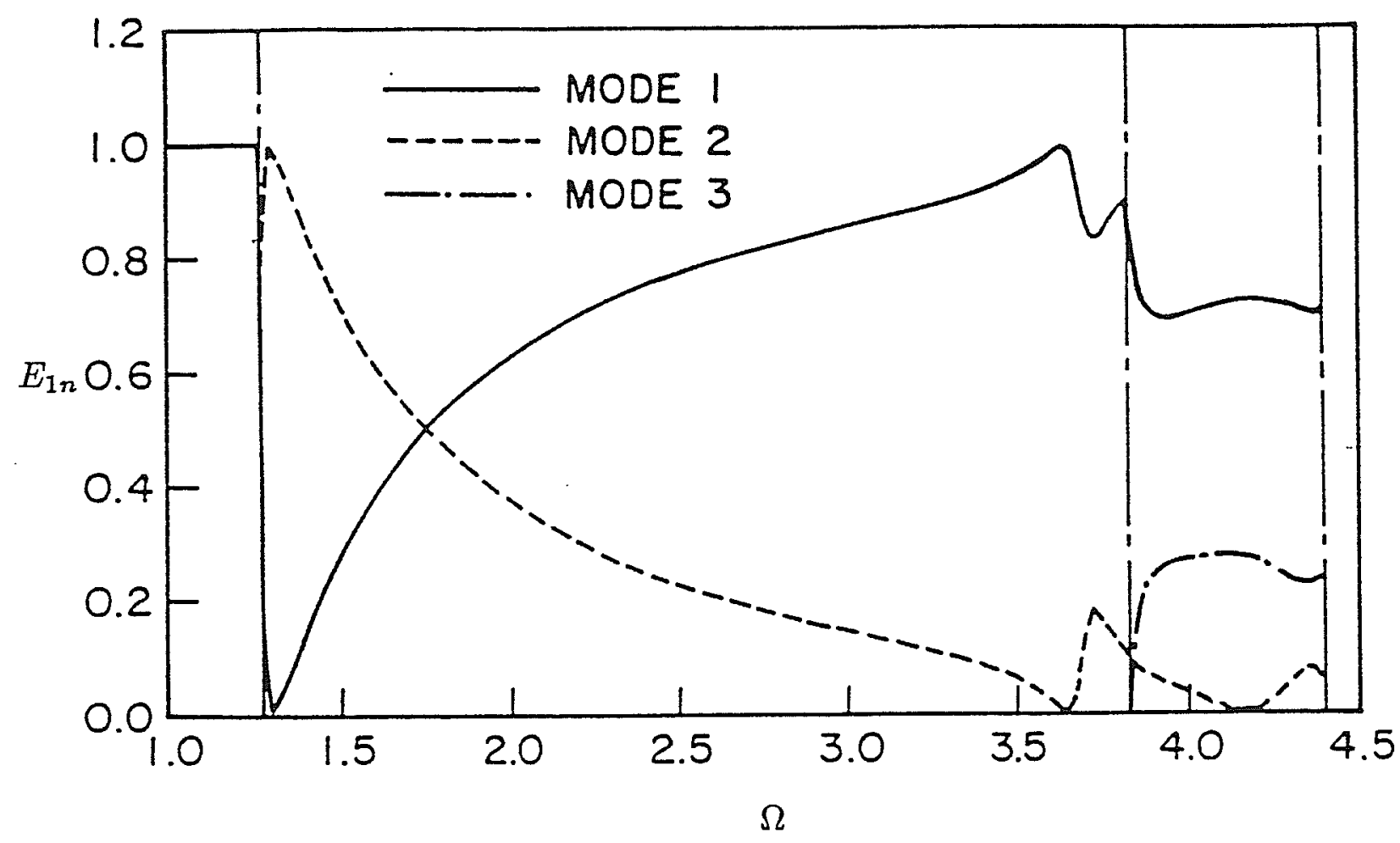

Figure 3.7 : The proportions of energy $E_{1 n}$ vs $\Omega$, for the 35-layer graphite-epoxy plate, due to first antisymmetric incident mode. 


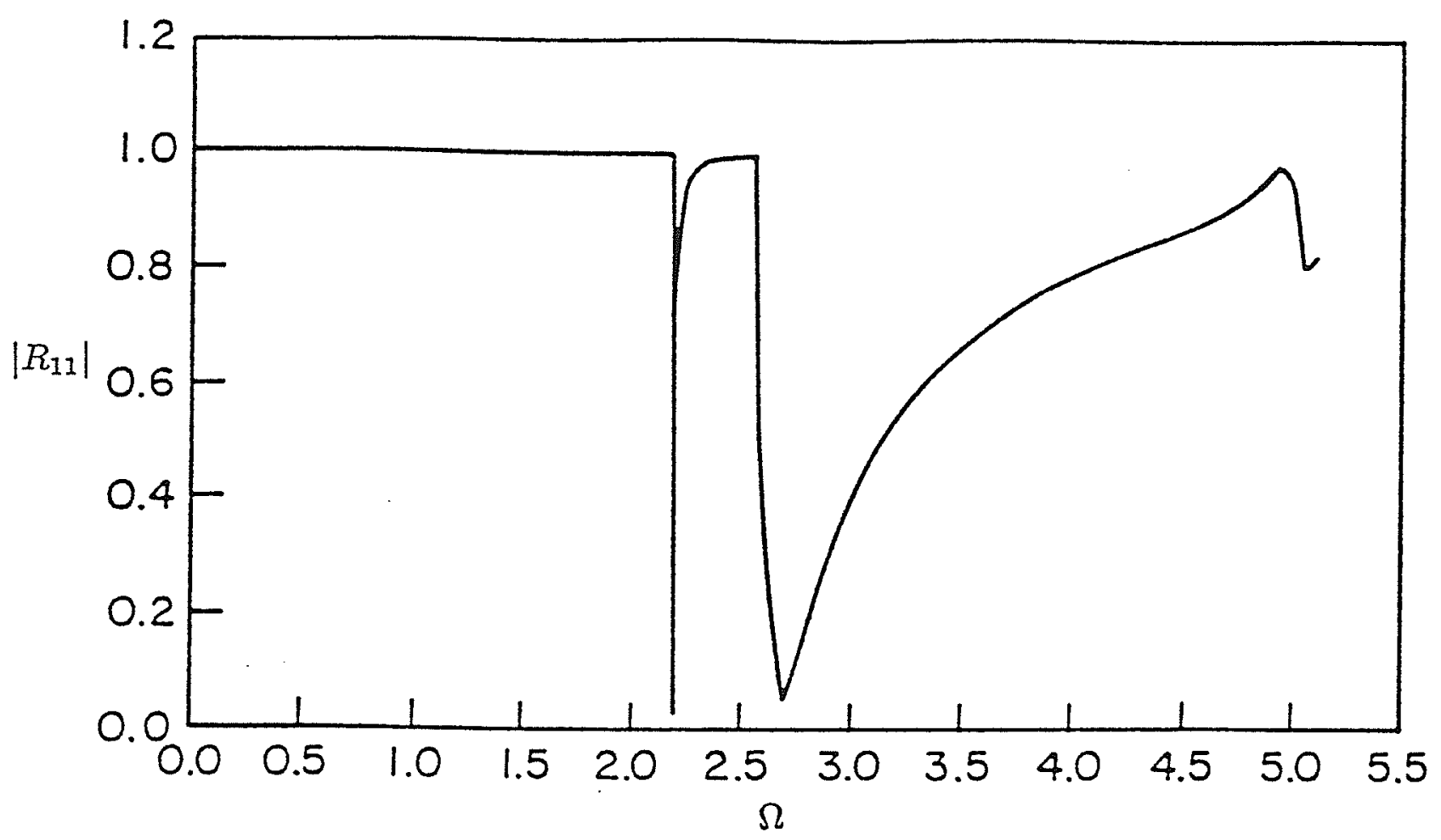

(a)

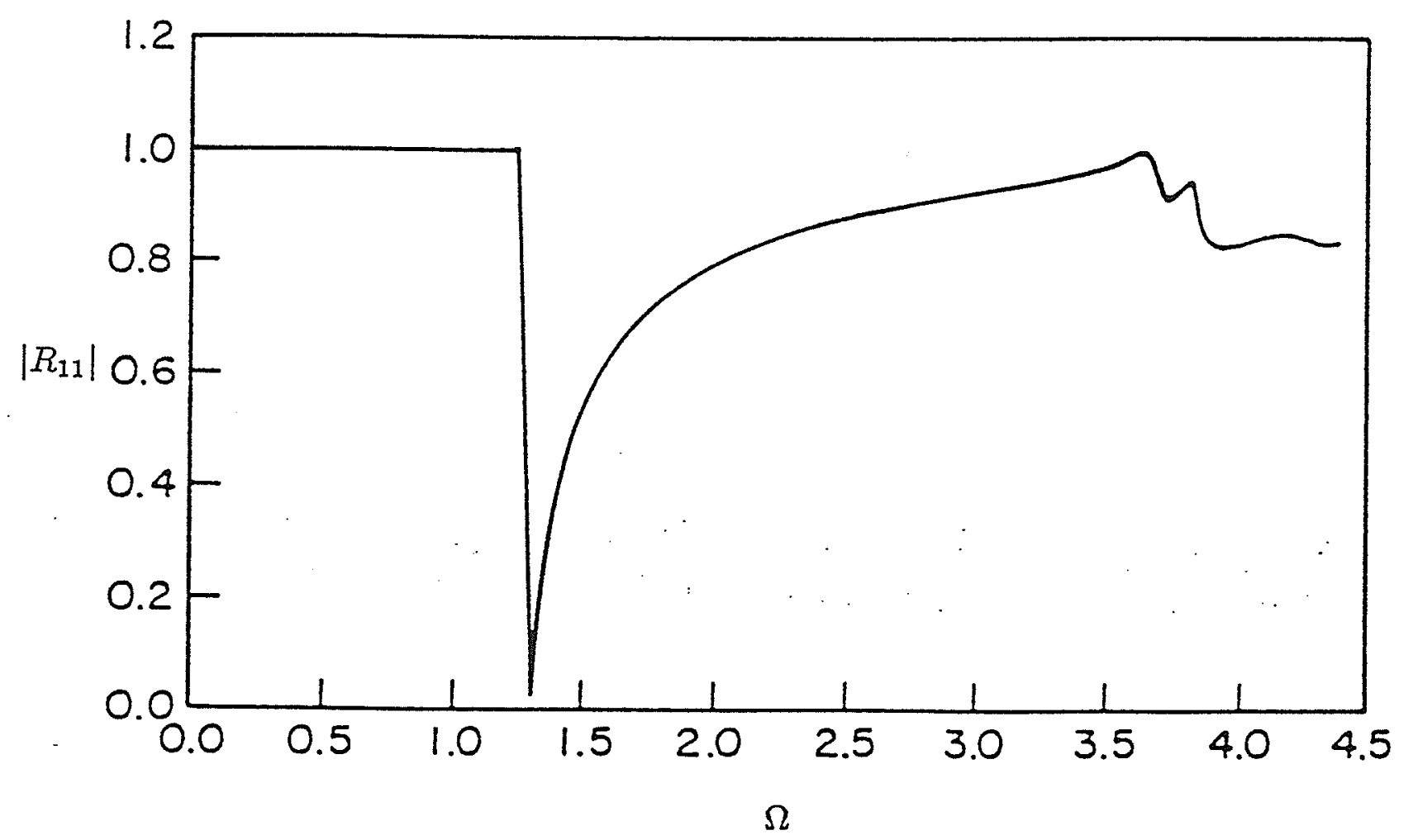

(b)

Figure 3.8: The reflection coefficient $\left|R_{11}\right|$ vs $\Omega$, for the 35-layer graphite-epoxy plate, (a) due to first symmetric incident mode,

(b) due to first antisymmetric incident mode. 
Table 3.1

Elastic sfiffnesses of $0^{\circ}$ and $90^{\circ}$ graphite-epoxy laminae (All stiffnesses are in units of $10^{11} \mathrm{~N} / \mathrm{m}^{2}$ ).

\begin{tabular}{|c|rrrr|}
\hline \hline lamina & $D_{11}$ & $D_{33}$ & $D_{13}$ & $D_{55}$ \\
\hline $0^{\circ}$ lamina & 1.6073 & 0.1392 & 0.0644 & 0.0707 \\
$90^{\circ}$ lamina & 0.1392 & 0.1392 & 0.0692 & 0.0350 \\
\hline \hline
\end{tabular}


Table 3.2

Variation of percentage error $\varepsilon$ in energy balance and reflection coefficient $\left|R_{11}\right|$ with number of modes, $M$, for homogeneous graphite-epoxy plate.

\begin{tabular}{|c|r|r|r|r|r|}
\hline \hline & & \multicolumn{2}{|c|}{$\varepsilon$} & \multicolumn{2}{|c|}{$\left|R_{11}\right|$} \\
\cline { 3 - 6 } Example & $M$ & Eq. $(3.20)$ & Eq. (3.24) & Eq. (3.20) & Eq. (3.24) \\
\hline & 4 & 51.354 & 10.836 & 0.170 & 0.245 \\
& 7 & 21.505 & 1.907 & 0.339 & 0.426 \\
Example 1 & 11 & 3.192 & 0.062 & 0.477 & 0.493 \\
$\Omega=4.0$ & 15 & 0.982 & 0.073 & 0.489 & 0.494 \\
& 19 & 0.325 & 0.140 & 0.493 & 0.494 \\
& 21 & 0.209 & 0.153 & 0.493 & 0.494 \\
& 23 & 0.155 & 0.160 & 0.494 & 0.494 \\
& 25 & 0.127 & 0.164 & 0.494 & 0.494 \\
\hline & 3 & 76.231 & 17.229 & 0.122 & 0.590 \\
& 6 & 58.006 & 7.023 & 0.253 & 0.621 \\
& 8 & 44.888 & 5.915 & 0.371 & 0.623 \\
Example 2 & 12 & 9.112 & 0.654 & 0.665 & 0.704 \\
& 15 & 6.423 & 0.830 & 0.680 & 0.704 \\
& 20 & 2.068 & 0.247 & 0.696 & 0.708 \\
& 25 & 1.212 & 0.223 & 0.702 & 0.709 \\
& 29 & 0.499 & 0.195 & 0.705 & 0.709 \\
\hline \hline
\end{tabular}




\section{Table 3.3}

Variation of percentage error $\varepsilon$ in energy balance and reflection coefficient $\left|R_{11}\right|$ with number of modes, $M$, for 35-layer graphite-epoxy plate.

\begin{tabular}{|c|r|r|r|r|r|}
\hline \hline & & \multicolumn{2}{|c|}{$\varepsilon$} & \multicolumn{2}{|c|}{$\left|R_{11}\right|$} \\
\cline { 2 - 6 } Example & $M$ & Eq. (3.20) & Eq. (3.24) & Eq. (3.20) & Eq. (3.24) \\
\hline & 3 & 91.947 & 28.291 & 0.188 & 0.735 \\
& 6 & 91.946 & 19.259 & 0.162 & 0.772 \\
& 10 & 92.265 & 19.892 & 0.109 & 0.807 \\
Example 3 & 12 & 84.150 & 16.481 & 0.102 & 0.741 \\
& 15 & 70.368 & 4.019 & 0.270 & 0.796 \\
& 18 & 54.948 & 1.669 & 0.436 & 0.807 \\
& 21 & 53.912 & 0.296 & 0.440 & 0.812 \\
& 24 & 54.996 & 0.172 & 0.441 & 0.811 \\
& 27 & 51.559 & 0.005 & 0.476 & 0.809 \\
& 30 & 51.519 & -0.001 & 0.477 & 0.809 \\
\hline & 3 & 89.365 & 13.817 & 0.083 & 0.674 \\
& 6 & 75.730 & 11.045 & 0.242 & 0.776 \\
& 10 & 74.344 & 9.437 & 0.330 & 0.786 \\
Example 4 4 & 12 & 63.389 & 6.579 & 0.469 & 0.803 \\
& 15 & 59.559 & 4.778 & 0.511 & 0.814 \\
& 17 & 23.118 & 1.466 & 0.776 & 0.827 \\
& 21 & 20.463 & 0.487 & 0.791 & 0.831 \\
& 24 & 20.471 & 0.388 & 0.792 & 0.831 \\
& 27 & 20.474 & 0.362 & 0.792 & 0.831 \\
\hline \hline
\end{tabular}




\section{$\mathrm{Ch}$ a p t e r 4}

\section{Wave Scattering by Cracks and Delaminations in laminated Composite Plates}

\subsection{General}

Defects and material degradation in structures made of composite materials have become a crucial problem for industries which use high-tech materials. Ultrasonic waves provide an efficient means of characterising such flaws. In order to use ultrasonic techniques to characterise flaws, it is neccessary to have a broad understanding of wave scattering by these flaws. Scattering by flaws in plate-like structures is a complicated phenomenon and the problem is made more difficult when the plate is a laminated composite one. In the past, considerable progress has been made towards understanding wave scattering by flaws in isotropic plates. However, information on scattering in anisotropic laminated plates is very limited. In this chapter, wave scattering by flaws in the form of cracks and delaminations in an infinite laminated composite plate is investigated. The geometry of the problem is depicted in Figures 4.1 and 4.2. The wave labeled as incident wave in Figure 4.1 may be thought of as being emitted by a source, which may be a line load applied perpendicular to the plate, located at a distance sufficiently far away from the flaw. Theoretically, any time variation in the incident wave can be considered using the Fourier Transform Technique. For simplicity, analysis presented here is restricted to time-harmonic incident waves. The incident waves propagate along the plate and strike the flaw in the plate resulting in generation of a scattered wave field. The scattered field consists of finitely many propagating wave modes and infinitely many nonpropagating and evanescent wave modes. The scattered field can be separated into reflected and transmitted waves. These waves, whose ampli- 
tudes contain information required for characterising the flaw, can be received by a transducer for post-processing. A hybrid method is presented in this chapter to solve the scattering problem and thereby to find theoretical numerical relationships between scattered wave amplitudes and the parameters of the crack/delamination.

The hybrid method is illustrated for the case of scattering by a symmetric normal edge crack (Figure 4.2(a)) and by a centrally located normal matrix crack that grows into delamination (Figure $4.2(\mathrm{~b})$ ). Reciprocity relations associated with the reflection and transmission coefficients and the principle of energy conservation are used as checks on the numerical accuracies. Numerical results of the scattering problem are presented for an isotropic plate, a homogeneous fiber-reinforced plate and for 8-layer and 35-layer laminated fiber-reinforced composite plates. Of partic-

ular interest are the results showing the dependence of conversion (reflection and transmission) coefficients on the extent of the delamination into which the normal central crack grows when it meets the adjacent lamina with $0^{\circ}$ fiber direction.

\subsection{Description of the Problem}

Time-harmonic wave scattering by a flaw (crack/delamination) in an infinite plate composed of perfectly bonded layers with possibly distinct mechanical properties and thickness is considered. It is assumed that the flaw is of constant geometry and of infinite length in $y$ direction. The two faces of the plate $z=0$ and $z=H$, and the surfaces of the flaw are traction-free. For simplicity in analysis, each layer is assumed to have transversely isotropic material properties. A train of time-harmonic waves excited at a location sufficiently far away from the flaw, propagates in a direction making an angle $\phi^{i n}$ with the negative $x$ direction and is incident upon the flaw. The incident wave, upon striking the flaw, generates a scattered wave field. 
In general, different scattered wave modes propagate in different directions in $x y$ plane. Since the waves are propagating in directions making arbitrary angles with fiber directions, the resulting wave motion, in general, is three dimensional. Denoting the particle displacement components in global $x, y, z$ directions by $u(x, y, z, t)$, $v(x, y, z, t), w(x, y, z, t)$, respectively, the governing equations that describe wave motion can be written as

$$
\begin{aligned}
& \frac{\partial \sigma_{x x}}{\partial x}+\frac{\partial \sigma_{x y}}{\partial y}+\frac{\partial \sigma_{x z}}{\partial z}=\rho \frac{\partial^{2} u}{\partial t^{2}} \\
& \frac{\partial \sigma_{x y}}{\partial x}+\frac{\partial \sigma_{y y}}{\partial y}+\frac{\partial \sigma_{y z}}{\partial z}=\rho \frac{\partial^{2} v}{\partial t^{2}} \\
& \frac{\partial \sigma_{x x}}{\partial x}+\frac{\partial \sigma_{y z}}{\partial y}+\frac{\partial \sigma_{z z}}{\partial z}=\rho \frac{\partial^{2} w}{\partial t^{2}}
\end{aligned}
$$

where $\sigma_{i j}$ are related to $\epsilon_{i j}$ by the constitutive relation given in equation (2.4), and $\epsilon_{i j}$ are related to displacement components by

$$
\begin{aligned}
\epsilon_{x x} & =\frac{\partial u}{\partial x}, \\
\epsilon_{y y} & =\frac{\partial v}{\partial y}, \\
\epsilon_{z z} & =\frac{\partial w}{\partial z} \\
\epsilon_{y z} & =\frac{1}{2} \gamma_{y z}=\frac{1}{2}\left(\frac{\partial v}{\partial z}+\frac{\partial w}{\partial y}\right), \\
\epsilon_{z x} & =\frac{1}{2} \gamma_{z x}=\frac{1}{2}\left(\frac{\partial u}{\partial z}+\frac{\partial w}{\partial x}\right), \\
\epsilon_{x y} & =\frac{1}{2} \gamma_{x y}=\frac{1}{2}\left(\frac{\partial v}{\partial x}+\frac{\partial u}{\partial y}\right) .
\end{aligned}
$$

The boundary conditions of the scattering problem are given by

$$
\sigma_{z x}=\sigma_{z y}=\sigma_{z z}=0 \quad \text { on the plate surfaces, }
$$

and

$$
\text { tractions on the surface of the flaw }=0 \text {. }
$$


The main objective here is to find a solution to the boundary value problem defined in equations (4.1)-(4.3). Since the plate is a laminated one, and in general the flaw is of arbitrary shape, finding an exact solution to the boundary value problem at hand is extremely difficult if not impossible. Therefore, an approximate solution through a hybrid technique is sought here.

\subsection{Hybrid Method}

The hybrid method combines finite element formulation in a bounded interior region of the plate with a wave function expansion representation in the exterior region. The interior region consists of the flaw and a small region of the plate surrounding the flaw. The regions are connected along vertical boundaries $\mathrm{B}^{+}$at $x=x^{+}$, and $\mathrm{B}^{-}$at $x=x^{-}$as shown in Figure 4.2. Continuity conditions for the displacement and interaction forces are imposed at the nodes lying on the boundaries. This results in a system of linear equations that is solved for the unknown wave function amplitudes. These amplitudes can be used to obtain boundary nodal displacements and in turn to obtain interior nodal displacements. This enables the determination of the wave field at any point of interest in the domain of the plate. It is noted here that the boundaries $\mathrm{B}^{+}$and $\mathrm{B}^{-}$need not be neccessarily vertical. One can have a boundary of arbitrary choice, however, choice of vertical boundaries make the algebra and the finite element mesh generation simple. In the sequel, formulation is presented in two parts. In the ensuing $\S 4.4$, the plane strain case is considered. In this case, waves are propagating either along or perpendicular to the fiber directions, and perpendicular to the axis of flaw, viz. $\phi^{\text {in }}=0^{\circ}$ and $\theta$ for each layer is equal to either $0^{\circ}$ or $90^{\circ}$. Note that the axis of flaw is in $y$ direction. However, since the orientation of the axis of the flaw is not known a priori it is not, in

general, possible to excite the incident wave in such a way that $\phi^{\mathrm{in}}=0^{\circ}$. Therefore, 
it is also necessary to consider a general case where $\phi^{\mathrm{in}} \neq 0^{\circ}$. This general case is considered in $\S 4.5$ wherein, the incident wave is propagating in a direction making an arbitrary angle with the $x$ - axis, and in general the fiber direction in each lamina may be at an arbitrary angle to the $x$ - axis. The time factor $\exp (-j \omega t)$ is omitted in what follows unless otherwise specifically mentioned.

\subsection{Plane Strain Case}

Consider the case in which the incident wave is the $p$-th propagating mode corresponding to the wavenumber $k_{p}$, propagating in the negative $x$ direction. The fibers in each lamina are assumed to be in either $x$ or $y$ direction. The only nonvanishing particle displacement components in this case are $u(x, z, t)$ and $w(x, z, t)$ in $x$ and $z$ directions, respectively, and in addition, the field quantities are independent of $y$.

\subsubsection{Wave Functions for Exterior Regions}

In the exterior regions $\mathrm{R}^{+}$and $\mathrm{R}^{-}$, the total wave field consists of a scattered field and the incident field. Since the incident wave is propagating either along or normal to the fiber directions, the resulting scattered waves will be propagating in the positive and negative $x$ directions. The scattered field is represented by wave functions expansion. Details of the methodology used to obtain wave functions for a laminated plate for plane strain case can be found in $\S 3.3$ of Chapter 3 . The procedure starts with dividing each layer into several sublayers so that the total number of sublayers through the thickness of the plate is $N$. When the incident wave strikes the flaw, a scattered wave field will be generated. Using the wave function expansion, the displacement vector of the scattered field, $\left\{q_{x}^{s+}\right\}$, in region 
$\mathrm{R}^{+}$at arbitrary $x$ can be written as

$$
\left\{q_{x}^{s+}\right\}=\sum_{m=1}^{M} A_{m}^{+}\left\{q_{m}\right\} \exp \left(j k_{m} x\right), \quad x \geq x^{+}
$$

where $A_{m}^{+}$is the amplitude of the $m$-th scattered mode in region $\mathrm{R}^{+} ; M$ is the total number of modes used in the wave function expansion; and $\left\{q_{m}\right\}$ is the $m$-th mode displacement vector defined in equation (3.6). The procedure for ordering $M$ number of significant roots $k$ is the same as that described in §3.3. Equation (4.4) gives the displacement vector of scattered wave field at the nodes on the boundary $\mathrm{B}^{+}$as

$$
\left\{q_{\mathrm{B}}^{\mathrm{s}+}\right\}=\left[G^{+}\right]\left\{D^{+}\right\}
$$

where

$$
\begin{aligned}
{\left[G^{+}\right] } & =\left[\begin{array}{llllll}
\left\{q_{1}\right\} & \left\{q_{2}\right\} & \cdots \cdots & \left\{q_{m}\right\} & \cdots \cdots & \left\{q_{M}\right\}
\end{array}\right], \\
\left\{D^{+}\right\}^{\mathrm{T}} & =\left\langle\begin{array}{llllll}
D_{1}^{+} & D_{2}^{+} & \cdots & D_{m}^{+} & \cdots & D_{M}^{+}
\end{array}\right\}, \\
D_{m}^{+} & =A_{m}^{+} \exp \left(j k_{m} x^{+}\right),
\end{aligned}
$$

$\left[G^{+}\right]$in equation (4.5) is a matrix of size $2(N+1)$ by $M$. The nodal force vector at the boundary $\mathrm{B}^{+}$due to scattered field can be formed as

$$
\left\{P_{\mathrm{B}}^{\mathrm{s}+}\right\}=\left[F^{+}\right]\left\{D^{+}\right\},
$$

where $\left[F^{+}\right]$is the matrix of nodal force mode vectors at the interfaces due to stress components $\sigma_{x x}$ and $\sigma_{x z}$. Note that $\left[F^{+}\right]=[F]$ defined in equation (3.15). Following a similar procedure at the boundary $\mathrm{B}^{-}$, the displacement and force vectors due to scattered field can be obtained as

$$
\begin{aligned}
\left\{q_{\mathrm{B}}^{\mathrm{s}-}\right\} & =\left[G^{-}\right]\left\{D^{-}\right\}, \\
\left\{P_{\mathrm{B}}^{\mathrm{s}-}\right\} & =\left[F^{-}\right]\left\{D^{-}\right\},
\end{aligned}
$$


respectively. It can be shown that $\left[G^{-}\right]$can simply be obtained from $\left[G^{+}\right]$after replacing each $x$ direction displacement component by the negative value of it. Similarly, $\left[F^{-}\right]$can be obtained from $\left[F^{+}\right]$after replacing each $x$ direction force component by the negative value of it. $\left\{D^{-}\right\}$is given by

$$
\left\{D^{-}\right\}^{\mathrm{T}}=\left\langle\begin{array}{llllll}
D_{1}^{-} & D_{2}^{-} & \cdots & D_{m}^{-} & \cdots & D_{M}^{-}
\end{array}\right\rangle
$$

where

$$
D_{m}^{-}=A_{m}^{-} \exp \left(-j k_{m} x^{-}\right), \quad m=1,2, \cdots, M
$$

in which, $A_{m}^{-}$is the amplitude of $m$-th scattered mode in region $\mathrm{R}^{-}$.

In a similar manner, the boundary displacement and force vectors for the incident wave can be constructed as

$$
\begin{array}{r}
\left\{q_{\mathrm{B}}^{\mathrm{in}+}\right\}=A_{p}^{\mathrm{in}}\left\{G_{p}^{-}\right\} \exp \left(-j k_{p} x^{+}\right), \\
\left\{q_{\mathrm{B}}^{\mathrm{in}-}\right\}=A_{p}^{\mathrm{in}}\left\{G_{p}^{-}\right\} \exp \left(-j k_{p} x^{-}\right), \\
\left\{P_{\mathrm{B}}^{\mathrm{in}+}\right\}=-A_{p}^{\mathrm{in}}\left\{F_{p}^{-}\right\} \exp \left(-j k_{p} x^{+}\right), \\
\left\{P_{\mathrm{B}}^{\mathrm{in}-}\right\}=A_{p}^{\mathrm{in}}\left\{F_{p}^{-}\right\} \exp \left(-j k_{p} x^{-}\right),
\end{array}
$$

where $\left\{G_{p}^{-}\right\}$and $\left\{F_{p}^{-}\right\}$are the $p$-th column of $\left[G^{-}\right]$and $\left[F^{-}\right]$matrices, respectively; and $A_{p}^{\text {in }}$ is the amplitude of the incident mode.

\subsubsection{Finite Element Model of Interior Region}

The interior region $R$ is modeled by finite elements. The crack tip singularity is modeled using six node quarterpoint triangular crack tip elements proposed by Barsoum (1976). The crack tip elements are surrounded with a layer of five node 
elements followed by conventional four node elements as shown in Figure 4.2c. The layer adjacent to the vertical boundaries $\mathrm{B}^{+}$and $\mathrm{B}^{-}$consists of five node elements with the midside node lying on the boundaries. This is done to increase the number of nodes lying on the boundaries.

The quarterpoint triangular elements have a built-in inverse square root stress singularity in them. The existence of an inverse square root singularity in the stress field around the tip of a crack imbedded in a homogeneous medium is well-known (see Fenner, 1976). Numerous studies on stress singularities at a crack located between two dissimilar media have been reported in the past (see, for example, Williams, 1959; Fenner, 1976; Ting and Hoang, 1984; Barsoum, 1988; Im, 1990). It has been reported that: when a crack is along the interface between two dissimilar media, the singular behaviour of the crack tip stress remains proportional to inverse square root of $r$ (the distance from the crack tip) but now has a pronounced oscillatory character; and when a crack meets an interface at right angles, the stress singularity is of the form $r^{-p}$, where $p$ depends on the elastic properties of two dissimilar media. In Ting and Hoang (1984), it has been shown that for typical high modulus graphite-epoxy composites $p$ is close to 0.5 for most combinations of ply-angles in the two dissimilar materials. The singularity at the tip of the crack affects only the local stress field near the crack tip. It should be noted here that the present scattering study is mainly concerned with the determination of far field (exterior region) scattered wave amplitudes. Therefore, in the present analysis, only the inverse square root singularity is incorporated in the finite element model near the crack tip.

The displacement vector, $\{u\}$, at a point within a typical finite element, 'e', is 
interpolated from the nodal displacements vector, $\left\{q^{e}\right\}$, as

$$
\{u\}=[N]\left\{q^{e}\right\}
$$

where $[N]$ contains interpolation functions (Zienkiewicz, 1977). The strain vector, $\{\epsilon\}$, at a point are related to displacement field through

$$
\{\epsilon\}=[L]\{u\}
$$

where $[L]$ is an operator matrix defined as

$$
[L]=\left[\begin{array}{cc}
\frac{\partial}{\partial x} & 0 \\
0 & \frac{\partial}{\partial z} \\
\frac{\partial}{\partial z} & \frac{\partial}{\partial x}
\end{array}\right]
$$

In view of equation (4.12), the strain vector can be expressed in terms of nodal displacements as

$$
\{\epsilon\}=[B]\left\{q^{e}\right\}
$$

where

$$
[B]=[L][N]
$$

An approximate equation governing the wave motion in the interior region may be obtained by minimizing the energy functional $\hat{\pi}$. In $(x, y, z)$ cartesian coordinate system, $\hat{\pi}$ (per unit length in $y$ direction) takes the form

$$
\hat{\pi}=\sum_{\mathrm{e}}\left[\frac{1}{2} \int_{A^{e}}\left(\{\bar{\epsilon}\}^{\mathrm{T}}[D]\{\epsilon\}-\rho \omega^{2}\{\bar{u}\}^{\mathrm{T}}\{u\}\right) d A\right]-\frac{1}{2}\left[\left\{\bar{q}_{\mathrm{B}}\right\}^{\mathrm{T}}\left\{P_{\mathrm{B}}\right\}+\left\{q_{\mathrm{B}}\right\}^{\mathrm{T}}\left\{\bar{P}_{\mathrm{B}}\right\}\right]
$$

where $\left\{q_{\mathrm{B}}\right\}$ and $\left\{P_{\mathrm{B}}\right\}$ are, respectively, the nodal displacement vector and interaction force vector (traction vector) corresponding to the nodes lying on the boundaries. In equation (4.17), the integral is over the area of element ' $e$ ' and $\sum_{e}$ implies 
summation over all the finite elements. It should be noted here that $\hat{\pi}$ is independent of time $t$ since $\overline{\exp (-j \omega t)} \exp (-j \omega t)$ is equal to unity. In view of equations (4.12)(4.16), and after the conventional assembly process in the finite element method, equation (4.17) can be written as

$$
\hat{\pi}=\frac{1}{2}\left\{\bar{q}_{\mathrm{T}}\right\}^{\mathrm{T}}[S]\left\{q_{\mathrm{T}}\right\}-\frac{1}{2}\left[\left\{\bar{q}_{\mathrm{B}}\right\}^{\mathrm{T}}\left\{P_{\mathrm{B}}\right\}+\left\{q_{\mathrm{B}}\right\}^{\mathrm{T}}\left\{\bar{P}_{\mathrm{B}}\right\}\right]
$$

where

$$
\begin{gathered}
\left\{q_{\mathrm{T}}\right\}^{\mathrm{T}}=\left\langle\left\{q_{\mathrm{I}}\right\}^{\mathrm{T}} \quad\left\{q_{\mathrm{B}}\right\}^{\mathrm{T}}\right\rangle \\
{[S]=\left[K_{\mathrm{T}}\right]-\omega^{2}\left[M_{\mathrm{T}}\right]=\left[\begin{array}{cc}
{\left[S_{\mathrm{II}}\right]} & {\left[S_{\mathrm{IB}}\right]} \\
{\left[S_{\mathrm{BI}}\right]} & {\left[S_{\mathrm{BB}}\right]}
\end{array}\right] .}
\end{gathered}
$$

In the above, $\left\{q_{\mathrm{I}}\right\}$ is the nodal displacement vector corresponding to interior nodes; and $\left[K_{\mathrm{T}}\right]$ and $\left[M_{\mathrm{T}}\right]$ are, respectively, the global stiffness and mass matrices of the interior region given by

$$
\begin{aligned}
& {\left[K_{\mathrm{T}}\right]=\cup[[k]],} \\
& {\left[M_{\mathrm{T}}\right]=\cup[[m]],}
\end{aligned}
$$

where $\cup$ stands for union or assembly, and

$$
\begin{aligned}
& {[k]=\int_{A^{e}}[B]^{\mathrm{T}}[D][B] d A,} \\
& {[m]=\int_{A^{c}} \rho[N]^{\mathrm{T}}[N] d A .}
\end{aligned}
$$

By minimizing the energy functional, one gets the governing equation of motion of whole interior region as

$$
\delta \hat{\pi}=\delta\left\{\bar{q}_{\mathrm{T}}\right\}^{\mathrm{T}}[S]\left\{q_{\mathrm{T}}\right\}-\delta\left\{\bar{q}_{\mathrm{B}}\right\}^{\mathrm{T}}\left\{P_{\mathrm{B}}\right\}=0 .
$$




\subsubsection{Global Solution}

The global solution is obtained by imposing the following continuity conditions on displacements and tractions at the mesh boundaries:

$$
\begin{gathered}
\left\{q_{\mathrm{B}}\right\}=\left\{q_{\mathrm{B}}^{\mathrm{in}}\right\}+\left\{q_{\mathrm{B}}^{\mathrm{s}}\right\}, \\
\left\{P_{\mathrm{B}}\right\}=\left\{P_{\mathrm{B}}^{\mathrm{in}}\right\}+\left\{P_{\mathrm{B}}^{\mathrm{s}}\right\},
\end{gathered}
$$

where

$$
\begin{aligned}
& \left\{q_{\mathrm{B}}^{\mathrm{in}}\right\}^{\mathrm{T}}=\left\langle\left\{q_{\mathrm{B}}^{\mathrm{in}-}\right\}^{\mathrm{T}} \quad\left\{q_{\mathrm{B}}^{\text {in }+}\right\}^{\mathrm{T}}\right\rangle, \\
& \left\{q_{\mathrm{B}}^{\mathrm{s}}\right\}^{\mathrm{T}}=\left\langle\left\{q_{\mathrm{B}}^{\mathrm{s}-}\right\}^{\mathrm{T}} \quad\left\{q_{\mathrm{B}}^{\mathrm{s}+}\right\}^{\mathrm{T}}\right\rangle, \\
& \left\{P_{\mathrm{B}}^{\mathrm{in}}\right\}^{\mathrm{T}}=\left\langle\left\{P_{\mathrm{B}}^{\mathrm{in}-}\right\}^{\mathrm{T}} \quad\left\{P_{\mathrm{B}}^{\mathrm{in}+}\right\}^{\mathrm{T}}\right\rangle, \\
& \left\{P_{\mathrm{B}}^{\mathrm{s}}\right\}^{\mathrm{T}}=\left\langle\left\{P_{\mathrm{B}}^{\mathrm{s}-}\right\}^{\mathrm{T}} \quad\left\{P_{\mathrm{B}}^{\mathrm{s}+}\right\}^{\mathrm{T}}\right\rangle \text {. }
\end{aligned}
$$

In equations (4.23), those quantities on the left hand side of the equal sign are from interior region while those on the right hand side are from the exterior region.

Using equations (4.5) and (4.8a) in equation (4.23a) and, in turn, in equation (4.22) results in

$$
\begin{aligned}
{\left[S_{\mathrm{II}}\right]\left\{q_{\mathrm{I}}\right\}+\left[S_{\mathrm{IB}}\right]\left\{q_{\mathrm{B}}\right\} } & =0 \\
{\left[\bar{G}_{1}\right]^{\mathrm{T}}\left(\left[S_{\mathrm{BI}}\right]\left\{q_{\mathrm{I}}\right\}+\left[S_{\mathrm{BB}}\right]\left\{q_{\mathrm{B}}\right\}\right) } & =\left[\bar{G}_{1}\right]^{\mathrm{T}}\left\{P_{\mathrm{B}}\right\}
\end{aligned}
$$

where

$$
\left[G_{1}\right]=\left[\begin{array}{cc}
{\left[G^{-}\right]} & {[0]} \\
{[0]} & {\left[G^{+}\right]}
\end{array}\right]
$$

Equation (4.25a) gives

$$
\left\{q_{\mathrm{I}}\right\}=-\left[S_{\mathrm{II}}\right]^{-1}\left[S_{\mathrm{IB}}\right]\left\{q_{\mathrm{B}}\right\}
$$


Substituting equation (4.27) in (4.25b) and making use of equations (4.23), one obtains

$$
\left[C_{1}\right]\left\{D_{1}\right\}=\left\{R_{1}\right\},
$$

where

$$
\begin{aligned}
{\left[C_{1}\right] } & =\left[\bar{G}_{1}\right]^{\mathrm{T}}\left(\left[S_{\mathrm{BB}}^{*}\right]\left[\bar{G}_{1}\right]-\left[F_{1}\right]\right), \\
\left\{D_{1}\right\}^{\mathrm{T}} & =\left\langle\left\{D^{-}\right\}^{\mathrm{T}} \quad\left\{D^{+}\right\}^{\mathrm{T}}\right\rangle, \\
\left\{R_{1}\right\} & =\left[\bar{G}_{1}\right]^{\mathrm{T}}\left(\left\{P_{\mathrm{B}}^{\mathrm{in}}\right\}-\left[S_{\mathrm{BB}}^{*}\right]\left\{q_{\mathrm{B}}^{\mathrm{in}}\right\}\right),
\end{aligned}
$$

in which,

$$
\begin{aligned}
{\left[S_{\mathrm{BB}}^{*}\right] } & =\left[S_{\mathrm{BB}}\right]-\left[S_{\mathrm{BI}}\right]\left[S_{\mathrm{II}}\right]^{-1}\left[S_{\mathrm{IB}}\right], \\
{\left[F_{1}\right] } & =\left[\begin{array}{cc}
{\left[F^{-}\right]} & {[0]} \\
{[0]} & {\left[F^{+}\right]}
\end{array}\right] .
\end{aligned}
$$

In the above, $\left[C_{1}\right]$ is a matrix of size $2 M$ by $2 M .\left\{D^{-}\right\}$and $\left\{D^{+}\right\}$are obtained by solving the linear system of equations (4.28). Amplitudes $A_{m}^{+}$and $A_{m}^{-}$are obtained from equations (4.6c) and (4.10), respectively. $\left\{q_{\mathrm{B}}\right\}$ is computed from equation (4.23a), making use of equations (4.5) and (4.8a). Then, $\left\{q_{\mathrm{I}}\right\}$ is given by equation (4.27).

The reflection coefficient $R_{p m}$ of the $m$-th reflected mode and transmission coefficient $T_{p m}$ of the $m$-th transmitted mode, due to $p$-th incident mode, are defined as

$$
\begin{aligned}
& R_{p m}=\frac{A_{m}^{+}}{A_{p}^{\text {in }}}, \\
& T_{p m}= \begin{cases}\frac{A_{m}^{-}}{A_{p}^{\text {in }}}, & m \neq p \\
\frac{A_{p}^{\text {in }}+A_{m}^{-}}{A_{p}^{\text {in }}}, & m=p .\end{cases}
\end{aligned}
$$




\subsubsection{Energy Conservation}

Reflected and transmitted energy is carried only by the propagating modes. Following the derivation of equation (3.32) in $\S 3.5$, the time-averaged value of the energy flux associated with the $n$-th reflected propagating mode through the plate cross section per unit length in $y$ direction, due to $p$-th incident mode, is given by

$$
I_{p n}^{+}=\omega\left|A_{p}^{\text {in }}\right|^{2}\left|R_{p n}\right|^{2} \vartheta_{n}
$$

where

$$
\vartheta_{n}=\operatorname{Im}\left[\left\{F_{n}^{+}\right\}^{\mathrm{T}}\left\{\bar{q}_{n}\right\}\right], \quad 1 \leq n \leq N_{\mathrm{pr}}
$$

in which, $N_{\mathrm{pr}}$ represents the number of propagating modes in the scattered field. Similarly, the energy flux of the $n$-th transmitted mode and the incident wave can be written, respectively, as

$$
\begin{aligned}
I_{p n}^{-} & =\omega\left|A_{p}^{\text {in }}\right|^{2}\left|T_{p n}\right|^{2} \vartheta_{n}, \\
I_{p}^{\text {in }} & =\omega\left|A_{p}^{\text {in }}\right|^{2} \vartheta_{p} .
\end{aligned}
$$

The percentage error in energy balance, $\varepsilon$, is defined as

$$
\varepsilon=\frac{100}{I_{p}^{\mathrm{in}}}\left[I_{p}^{\mathrm{in}}-\sum_{n=1}^{N_{\mathrm{pr}}}\left(I_{p n}^{+}+I_{p n}^{-}\right)\right]
$$

Application of the principle of conservation of energy to the close region $\mathrm{R}$ bounded by $x=x^{+}, \quad x=x^{-}$, and top and bottom plate surfaces shows that $\varepsilon$ should be zero. This condition is used to assess the numerical accuracy of the analysis. 


\subsubsection{Reciprocity Relations}

Application of the elastodynamic reciprocity theorem (Achenbach, 1973; Auld, 1973 ; Tan and Auld, 1980) to the region $\mathrm{R}$ results in

$$
\begin{aligned}
& R_{p n} \varsigma_{n}=R_{n p} \varsigma_{p}, \\
& T_{p n} \varsigma_{n}=T_{n p} \varsigma_{p},
\end{aligned}
$$

where

$$
\varsigma_{n}=-2\left\{F_{n}^{-}\right\}^{\mathrm{T}}\left\{q_{n}\right\}, \quad 1 \leq n \leq N_{\mathrm{pr}}
$$

Details of the derivation of equations (4.36) are given in Appendix D.

A close examination of the elements of the matrices $\left[P_{i}\right],\left[G^{+}\right],\left[F^{+}\right]$, and $\left[F^{-}\right]$ reveals that $\varsigma_{n}$ is related to $\vartheta_{n}$ by

$$
\varsigma_{n}=2 j \vartheta_{n}
$$

Let $E_{p n}^{+}$be the proportion of incident energy transfered into the $n$-th reflected mode. Then

$$
E_{p n}^{+}=\frac{I_{p n}^{+}}{I_{p}^{\text {in }}}=\left|R_{p n}\right|^{2} \frac{\vartheta_{n}}{\vartheta_{p}}
$$

Similarly,

$$
E_{n p}^{+}=\frac{I_{n p}^{+}}{I_{n}^{\text {in }}}=\left|R_{n p}\right|^{2} \frac{\vartheta_{p}}{\vartheta_{n}} .
$$

Equations (4.36), and (4.38)-(4.40) lead to

$$
E_{p n}^{+}=E_{n p}^{+}
$$


In a similar manner, it can be shown that, for the transmitted modes,

$$
E_{p n}^{-}=E_{n p}^{-} .
$$

Reciprocity relations in equations (4.36), (4.41), and (4.42) serve as numerical checks on computations.

\subsection{General Case}

This case occurs when the incident wave is not propagating in a direction normal to the axis of the flaw. In addition, the fibers in each lamina may be at an arbitrary angle to the $x$ - axis (see Figure 4.1). Thus, the resulting wave motion will have all three particle displacement components. In this case, the scattered waves are propagating in several directions in $x y$ plane. Direction $O x^{\prime}$ in Figure 4.1 represents a typical direction of propagation of a scattered wave mode. Let $k^{(0)}$ be the wavenumber of the incident wave in the direction of propagation. Thus, $k^{(o)}$ should be one of the admissible real roots of the dispersion equation for off-axis propagation case described in $\S 2.3 .3$. As mentioned in $\S 2.3 .3$, superscript (o) here refers to off-axis propagation. Since the flaw extends to infinity in $y$-direction, no scattering will occur in that direction. Only the $x$ direction incident wave component is subjected to scattering by the flaw. Thus, each of the scattered wave modes will have a constant wavenumber $\zeta_{0} \quad\left(=k^{(o)} \sin \phi^{i n}\right)$ in the negative $y$ direction. Therefore, for time-harmonic waves, $y$ and $t$ variation can be separated out as

$$
\left\{\begin{array}{c}
u(x, y, z, t) \\
v(x, y, z, t) \\
w(x, y, z, t)
\end{array}\right\}=\left\{\begin{array}{c}
\hat{u}(x, z) \\
\hat{v}(x, z) \\
\hat{w}(x, z)
\end{array}\right\} \exp \left[-j\left(\zeta_{0} y+\omega t\right)\right]
$$

In the subsequent analysis, the common factor $\exp \left[-j\left(\zeta_{0} y+\omega t\right)\right]$ is dropped unless otherwise specifically mentioned. 


\subsubsection{Wave Functions for Exterior Regions}

The scattered field in the exterior regions $\mathrm{R}^{+}$and $\mathrm{R}^{-}$is represented by wave function expansion. As in the plane strain case, wave functions are obtained by considering the wave propagation in an infinite plate. The procedure starts with dividing each layer into several sublayers. For the $i$-th sublayer, the wavenumbers in $X$ and $Y$ directions are, respectively, given by

$$
K^{(g)}=k \cos \theta+\zeta_{0} \sin \theta
$$

and

$$
L^{(g)}=k \sin \theta-\zeta_{0} \cos \theta
$$

where $k$ now denotes the $x$ direction wavenumber of a typical wave mode, and the superscript $(g)$ has been used to represent the general case. After a careful examination of Figure 4.1, one finds that when every wave mode has a predetermined constant wavenumber $\left(\zeta_{0}\right)$ in the negative $y$ direction, analytical dispersion equation can be obtained from equation (2.46) after replacing $K$ by $K^{(g)}$ and $L$ by $L^{(g)}$. Thus, the dispersion equation for general case can be written as

$$
f^{(g)}(\omega, k)=\left|\begin{array}{lll}
P_{31}^{(g)} & P_{32}^{(g)} & P_{36}^{(g)} \\
P_{41}^{(g)} & P_{42}^{(g)} & P_{46}^{(g)} \\
P_{51}^{(g)} & P_{52}^{(g)} & P_{56}^{(g)}
\end{array}\right|=0 .
$$

where $P_{m n}^{(g)}$ represents elements of the global propagator matrix for general case. $P_{m n}^{(g)}$ are obtained from $P_{m n}^{(o)}$ after replacing $K$ by $K^{(g)}$ and $L$ by $L^{(g)}$. The propagator matrix $\left[P_{i}^{(g)}\right]$ for the $i$-th sublayer is obtained in a similar manner. For a particular value of $\omega$, transcendental equation (4.45) will have a finite number of real roots and an infinite number of imaginary and complex roots for $k$. Roots $k$ 
are found by employing Mullers's method with initial guesses obtained via stiffness method II. The procedure for wavenumber determination and ordering of $M$ number of significant roots $k$ are similar to those given in $\S 3.3$. It may be mentioned here that since $\zeta_{0}$ fixed, orientation of $x^{\prime}$ in Figure 4.1(b) will vary with different $k$.

For the general case, equation (2.43) and (2.45) take the forms

$$
\left\{B_{i+1}^{(g)}\right\}=\left[P_{i}^{(g)}\right]\left\{B_{i}^{(g)}\right\},
$$

and

$$
\left[\begin{array}{lll}
P_{31}^{(g)} & P_{32}^{(g)} & P_{36}^{(g)} \\
P_{41}^{(g)} & P_{42}^{(g)} & P_{46}^{(g)} \\
P_{51}^{(g)} & P_{52}^{(g)} & P_{56}^{(g)}
\end{array}\right]\left\{\begin{array}{c}
\hat{u}_{1} \\
\hat{v}_{1} \\
\hat{w}_{1}
\end{array}\right\}=\left\{\begin{array}{l}
0 \\
0 \\
0
\end{array}\right\}
$$

respectively. In equation (4.46), $\left\{B_{i}^{(g)}\right\}$ represents the displacements and stress components at $z=z_{i}$ as

$$
\left\{B_{i}^{(g)}\right\}^{\mathrm{T}}=\left\langle\begin{array}{llllll}
\hat{u}_{i} & \hat{v}_{i} & \hat{\sigma}_{z z i} & \hat{\sigma}_{z x i} & \hat{\sigma}_{y z i} & \hat{w}_{i}
\end{array}\right\rangle
$$

Traction-free conditions at interface 1 , and equation (4.47) give the components of the $m$-th mode eigenvector at interface 1 , as

$$
\left\{B_{1 m}^{(g)}\right\}^{\mathrm{T}}=\left\langle\begin{array}{llllll}
1 & p_{1} & 0 & 0 & 0 & p_{2}
\end{array}\right\rangle,
$$

where

$$
\begin{aligned}
& p_{1}=\frac{P_{41}^{(g)} P_{36}^{(g)}-P_{31}^{(g)} P_{46}^{(g)}}{P_{32}^{(g)} P_{46}^{(g)}-P_{36}^{(g)} P_{42}^{(g)}}, \\
& p_{2}=\frac{P_{31}^{(g)} P_{42}^{(g)}-P_{42}^{(g)} P_{32}^{(g)}}{P_{32}^{(g)} P_{46}^{(g)}-P_{36}^{(g)} P_{42}^{(g)}} .
\end{aligned}
$$

It is to be noted that $p_{1}$ and $p_{2}$ should be obtained from equations (4.50) for the $m$-th root of $k$ (ie. for $k_{m}$ ). Then applying equation (4.46) at successive interfaces, 
the $m$-th mode eigenvector (values of wave functions at discrete interfaces) can be obtained as

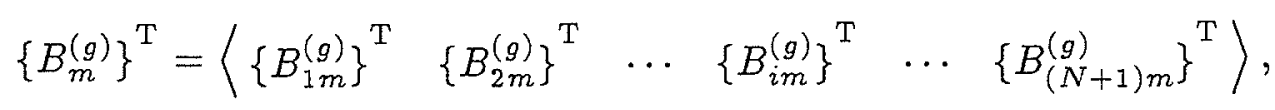

where

$$
\begin{aligned}
& \left\{B_{i m}^{(g)}\right\}^{\mathrm{T}}=\left\langle\begin{array}{llllll}
\hat{u}_{i m} & \hat{v}_{i m} & \hat{\sigma}_{z z i m} & \hat{\sigma}_{z x i m} & \hat{\sigma}_{y z i m} & \hat{w}_{i m}
\end{array}\right\rangle, \\
& i=1,2, \cdots \cdots, N+1 ; \quad m=1,2, \cdots \cdots, M .
\end{aligned}
$$

In the above, $\hat{u}_{i m}, \hat{v}_{i m}$, and $\hat{w}_{i m}$ are the displacement components, and $\hat{\sigma}_{z z i m}$, $\hat{\sigma}_{z x i m}$, and $\hat{\sigma}_{y z i m}$ are the stress components, at the $i$-th interface corresponding to the $m$-th mode (after suppressing the factor $\left.\exp \left[-j\left(\zeta_{0} y+\omega t\right)\right]\right)$. As mentioned previously, if the problem under consideration is symmetric or antisymmetric, only a half-thickness of the plate may be modeled using appropriate boundary conditions at the middle plane of the plate.

Following the analysis presented in $\S 4.4 .1$, the displacement vectors of the scattered wave field at the boundaries $\mathrm{B}^{+}$and $\mathrm{B}^{-}$can still be represented by equation (4.5) and (4.8a), respectively, with following modifications: $\left[G^{+}\right]$is now replaced by

$$
\left[G^{+}\right]=\left[\begin{array}{llllll}
\left\{q_{1}^{(g)}\right\} & \left\{q_{2}^{(g)}\right\} & \cdots & \left\{q_{m}^{(g)}\right\} & \cdots & \left\{q_{M}^{(g)}\right\}
\end{array}\right]
$$

where

$$
\begin{aligned}
& \left\{q_{m}^{(g)}\right\}^{\mathrm{T}}=\left\langle\hat{u}_{1 m} \quad \hat{v}_{1 m} \quad \hat{w}_{1 m} \quad \cdots \cdots \cdot \hat{u}_{i m} \quad \hat{v}_{i m} \quad \hat{w}_{i m} \quad \cdots \cdots\right. \\
& \left.\cdots \hat{u}_{(N+1) m} \quad \hat{v}_{(N+1) m} \quad \hat{w}_{(N+1) m}\right\rangle ;
\end{aligned}
$$

and $\left[G^{-}\right]$is constructed in a similar manner. 
In view of equations $(2.36),(2.37 \mathrm{a}),(2.37 \mathrm{~b}),(2.37 \mathrm{f}),(2.39)$, and $(2.40)$, omitting details of algebra, the $\hat{\sigma}_{x x}$ and $\hat{\sigma}_{x y}$ components of stresses within the $i$-th sublayer can be expressed as

$$
\left\{\begin{array}{c}
\hat{\sigma}_{x x} \\
\hat{\sigma}_{x y}
\end{array}\right\}=[U]\left\{\begin{array}{c}
\hat{u} \\
\hat{v} \\
\hat{\sigma}_{z z}
\end{array}\right\}
$$

In equation (4.55), the 2 by 3 matrix $[U]$ is given by

$$
[U]=\left[T_{3}\right]\left[T_{4}^{(g)}\right]\left[R^{(g)}\right]^{-1}\left[T_{2}\right]^{\mathrm{T}}
$$

where $\left[R^{(g)}\right]$ is obtained from $[R]$ in Appendix B after replacing $K$ by $K^{(g)}$ and $L$ by $L^{(g)} ;\left[T_{2}\right]$ is given in Appendix $\mathrm{B}$; and $\left[T_{3}\right]$ and $\left[T_{4}^{(g)}\right]$ are defined in Appendix E. Since $\hat{u}_{i m}, \hat{v}_{i m}$, and $\hat{\sigma}_{z z i m}$ for the $m$-th mode are known from equation (4.51), equation (4.55) can be used to compute $\hat{\sigma}_{x x i m}$ and $\hat{\sigma}_{x y i m}$ at each interface. It should be noted that $\hat{\sigma}_{x x i m}$ and $\hat{\sigma}_{x y i m}$ are discontinuous at the interfaces between sublayers of different material properties. The nodal force vectors due to scattered wave field at the boundaries $\mathrm{B}^{+}$and $\mathrm{B}^{-}$are now given, respectively, by equations (4.7) and (4.8a) where $\left[F^{+}\right]$is replaced by

$$
\left[F^{+}\right]=\left[\begin{array}{llllll}
\left\{F_{1}^{(g)}\right\} & \left\{F_{2}^{(g)}\right\} & \cdots & \left\{F_{m}^{(g)}\right\} & \cdots & \left\{F_{M}^{(g)}\right\}
\end{array}\right]
$$

where

$$
\begin{aligned}
& \left\{F_{m}^{(g)}\right\}^{\mathrm{T}}=\left\langle F_{1 m}^{(g) x} \quad F_{1 m}^{(g) y} \quad F_{1 m}^{(g) z} \quad \cdots \quad F_{i m}^{(g) x} \quad F_{i m}^{(g) y}, F_{i m}^{(g) z} \quad \cdots\right. \\
& \left.\cdots \quad F_{(N+1) m}^{(g) x} \quad F_{(N+1) m}^{(g) y} \quad F_{(N+1) m}^{(g) z}\right) ;
\end{aligned}
$$

and $\left[F^{-}\right]$is constructed in a similar manner. The explicit forms of $F_{i m}^{(g) x}, F_{i m}^{(g) y}$, and $F_{i m}^{(g) z}$ are given in Appendix F. 
In a similar manner, boundary nodal displacement and force vectors corresponding to the incident wave are given by equations (4.11).

\subsubsection{Finite Element Model of Interior Region}

The procedure of finite element formulation for interior region $\mathrm{R}$ for general case is very similar to that of for plane strain case given in $\S 4.4 .2$. Therefore, no detailed formulation is presented here. A point to note is that operator matrix $[L]$ in equation (4.14) now assumes the form

$$
[L]=\left[\begin{array}{ccc}
\frac{\partial}{\partial x} & 0 & 0 \\
0 & -j \zeta_{0} & 0 \\
0 & 0 & \frac{\partial}{\partial z} \\
0 & \frac{\partial}{\partial z} & -j \zeta_{0} \\
\frac{\partial}{\partial z} & 0 & \frac{\partial}{\partial x} \\
-j \zeta_{0} & \frac{\partial}{\partial x} & 0
\end{array}\right]
$$

It is interesting to note here that the energy functional, $\hat{\pi}$, for general case is independent of $y$ and $t$. This is because terms involving $y$ and $t$ in $\hat{\pi}$ appear as $\overline{\exp \left[-j\left(\zeta_{0} y+\omega t\right)\right]} \exp \left[-j\left(\zeta_{0} y+\omega t\right)\right]$ which is equal to unity. The fact that $\hat{\pi}$ is not dependent on $y$ reveals that a two dimensional finite element discretization in $x z$ plane can be carried out.

\subsubsection{Global Solution}

The global solution is obtained by imposing the continuity of total (incident plus scattered) displacements and tractions on the boundaries $\mathrm{B}^{+}$and $\mathrm{B}^{-}$. These

lead to linear algebraic equations for governing the amplitudes $A_{m}^{+}$and $A_{m}^{-}$. Since formulation is very similar to the plane case given in $\S 4.4 .3$, no details are produced here. Note that the final system of linear algebraic equations involve $3 M$ equations as compared to $2 M$ equations in plane strain case. The reflection and transmission coefficients are given by equations (4.31). 


\subsubsection{Energy Conservation and Reciprocity Relations}

Application of the principle of energy conservation to the close volume bounded by $x=x^{+}, x=x^{-}, y=y^{+}, y=y^{-}$, and top and bottom plate surfaces shows that the percentage error in energy balance $\varepsilon$ defined in equation (4.35) should be theoretically zero. $y^{+}$and $y^{-}$here denote two arbitrary $y$-coordinates. Equation (4.35) should be used to compute $\varepsilon$ for general case with the understanding that $\vartheta_{n}$ in equation (4.33) has to be replaced by

$$
\vartheta_{n}=\operatorname{Im}\left[\left\{F_{n}^{(g)}\right\}^{\mathrm{T}}\left\{\bar{q}_{n}^{(g)}\right\}\right], \quad 1 \leq n \leq N_{\mathrm{pr}}
$$

The reciprocity relations for transmitted wave field are given by equations $(4.36 \mathrm{~b})$ and (4.42) where $\varsigma_{n}$ is now given by

$$
\varsigma_{n}=-2\left\{F_{n}^{(g)-}\right\}^{\mathrm{T}}\left\{q_{n}^{(g)}\right\}, \quad 1 \leq n \leq N_{\mathrm{pr}} .
$$

where $\left\{F_{n}^{(g)-}\right\}$ is the $n$-th column of $\left[F^{(g)-}\right]$. Reciprocity relations given in equations (4.36a) and (4.41) take a slightly different form for general case. Details of this can be found in Appendix D.

\subsection{Numerical Results and Discussion}

The hybrid method developed in previous sections is used to obtain numerical results of the scattering problem for following five examples:

Example 1 - a homogeneous isotropic plate with Poisson's ratio, $\nu=0.31$. The flaw considered is a symmetric normal edge crack, the geometry of which is depicted in Figure 4.2(a).

Example 2 - a homogeneous graphite-epoxy plate with fibers aligned along the $x$-axis $\left(0^{\circ}\right)$. The flaw is a symmetric normal edge crack. 
Example 3 - a 35-layer graphite-epoxy cross-ply laminated plate with $90^{\circ} / 0^{\circ} / \cdots / 90^{\circ} / 0^{\circ} / 90^{\circ} / \cdots / 0^{\circ} / 90^{\circ}$ configuration. The flaw considered is a symmetric normal edge crack.

Example 4 - an 8-layer graphite-epoxy cross-ply laminated plate with $0^{\circ} / 90^{\circ} / 0^{\circ} / 90^{\circ} / 90^{\circ} / 0^{\circ} / 90^{\circ} / 0^{\circ}$ configuration. The flaw considered is that shown in Figure 4.2(b). Note that for this flaw $b_{2}$ is equal to zero until $b / b_{1}$ is equal to 1.0 , and when the normal crack grows into delamination (i.e. $b_{2}>0$ ) $b$ is equal to $b_{1}+b_{2}$.

Example 5 - The configuration of the plate is same as in example 4, but the flaw considered is a symmetric normal edge crack.

The material properties of graphite-epoxy layers for examples 2-5 are as given in §2.6. Since in each of the above examples, the geometry of the plate and flaw is symmetric with respect to the midplane of the plate, the scattered field consists of either symmetric or antisymmetric modes depending on whether the incident mode is a symmetric or an antisymmetric one. Thus only half-thickness of the plate need be modeled in the analysis. The following nondimensionalization has been used for frequency throughout this section:

$$
\begin{aligned}
& \text { Example } 1-\Omega=\frac{\omega H}{2 \sqrt{\mu / \rho}} ; \mu=\text { shear modulus; } \\
& \text { Example 2-5 }-\Omega=\frac{\omega H}{2 \sqrt{C_{55} / \rho}} .
\end{aligned}
$$

Three checks are made to validate the numerical calculations: (1) for an incident wave without any crack in the interior region, the resulting scattered field should be zero; (2) the percentage error in energy balance, $\varepsilon$, should be zero; and (3) the reciprocity relations should be satisfied. These three checks were satisfied with 
negligible errors for the results to be presented in $\S 4.6 .1$ for plane strain case, and $\S 4.6 .2$ for general case.

The total number of sublayers, $N$, used to compute the wave functions for the exterior region plays an important role in the accuracy of the analysis. In each of the examples considered a suitable value for $N$ was chosen after computing $\vartheta_{n}$ by increasing the value of $N$ at few selected lower, intermediate and higher frequencies in the frequency range of interest, until converged values were obtained for $\vartheta_{n}$. The finite element mesh was automatically generated with arbitrary normalized crack length, $a /(H / 2)$, at normalized crack length increment of $1 / 20$ in examples 1 and 2 , and $1 / 35$ in examples 3 . In example 4 , for scattering by the normal central crack, the finite element mesh was automatically generated with arbitrary normalized crack length, $b / b_{1}$, at normalized crack length increment of $1 / 8$. To simplify the automatic generation of the mesh, the finite elements were taken to be rectangular (except near the crack tip) with equal heights and equal widths.

In order to choose suitable values for the boundary coordinates, $x^{+}$, and $x^{-}$, numerical results were obtained by increasing the overall width of the mesh, starting with a smaller width. It was found that any width above a threshold mesh width can satisfy the three checks mentioned in the previous paragraph with negligible errors. However, as the mesh width becomes larger, the number of finite elements involved also becomes larger thus effectively increasing the number of degrees of freedoms in the finite element analysis. The mesh boundaries were chosen here as $x^{-} /(H / 2)=-0.20$, and $x^{+} /(H / 2)=0.20$ except when $b / b_{1}$ is greater than 1.0 in example 4. The interior region enclosed by these mesh boundary coordinates consisted of 8 columns of finite elements symmetrically located about the $x=0$ plane. For the delamination crack $\left(b / b_{1}>1.0\right.$ in example 4$)$ the mesh boundaries were chosen as $x^{-} /(H / 2)=-7 / 32$, and $x^{+} /(H / 2)=7 / 32$, and there were 14 
columns of finite elements symmetrically located about the $x=0$ plane. The meshes contained: 20 rows of finite elements in examples 1,2 , and $5 ; 35$ rows of finite elements in example 3 ; and 32 rows of finite elements in example 4 . In all five examples, the number of modes, $M$, employed in the wave function expansion were in the range 15 to 21 .

\subsubsection{Plane Strain Case}

\subsubsection{Normal Edge Crack}

The numerical results of the scattering problem for example 1-3, for plane strain case are presented in this section. A comparison of numerical results for the reflection coefficient $R_{11}$ due to first symmetric incident mode, with those of Koshiba et al. (1984) for example 1 are shown in Figure 4.3 where $\Omega$ is $\pi / 2, N_{\mathrm{pr}}$ is one, and circles indicate results of Koshiba et al.. The frequency spectrum for this example can be found in Mindlin (1960). For the results presented, $|\varepsilon|$ was less than $0.005 \%$. The excellent comparison of results serves to verify the computer code developed here to model the scattering problem.

Variation of the reflection coefficient $R_{11}$ (due to first symmetric incident mode) with normalized crack length for $\Omega=2.0$ in example 2 are shown in Figure 4.4. The frequency spectrum for this problem was shown in Figure 3.2(a). It can be seen from the frequency spectrum that at $\Omega=2.0$, the scattered field consists of only one symmetric propagating mode. For these results, $|\varepsilon|$ was less than $0.006 \%$. Figure 4.5 shows the variation of reflection coefficients with normalized crack length for $\Omega=4.0$ in example 2, when the incident mode is the first symmetric one. At this frequency, the scattered field consists of three symmetric propagating modes. It was found that $|\varepsilon|$ was less than $0.35 \%$. Comparison of Figure 4.4 with Figure 4.5 shows that 
the variation of $R_{11}$ is quite different in these two cases. This is due to the fact that at $\Omega=2.0$ the energy of incident wave is shared by only one reflected propagating mode and one transmitted propagating mode whereas at $\Omega=4.0$ the energy of the incident wave is shared by three reflected and three transmitted propagating wave modes. It is seen from Figure 4.5 that when the crack length approches the special case of through-thickness crack (ie. free end reflection), $R_{12}$ approaches zero resulting in the reflected energy being shared by only the first and third reflected propagating modes.

Numerical results of reflection coefficients for example 3 at $\Omega=1.2$ and 2.5, when the incident mode is the first symmetric mode, are shown in Figures 4.6 and 4.7 , respectively. The frequency spectrum for this example was presented in Figure 3.2(b). $N_{\mathrm{pr}}$ was 1 at $\Omega=1.2$, and 2 at $\Omega=2.5$. The maximum value of $|\varepsilon|$ was $0.003 \%$ at $\Omega=1.2$ and $0.09 \%$ at $\Omega=2.5$. In $\S 2.6 .4$, it was found that when the number of layers in a cross-ply laminated plate exceeds a threshold number, the plate can be modeled as an effective homogeneous anisotropic plate. This limit for the graphite-epoxy laminated plate considered here was found to be about 35 . In Figures 4.6 and 4.7, the results of reflection coefficient for this effective plate are shown by triangles. It can be seen that the results for the effective plate are in close agreement with the results for the 35-layer plate. Finally, in Figures 4.8 and 4.9 , the results of the reflection coefficients for example 3 at $\Omega=1.2$ and 2.5 , when the incident mode is the first antisymmetric mode, are shown. The scattered field consists of one antisymmetric propagating mode at $\Omega=1.2$ and two antisymmetric propagating modes at $\Omega=2.5$. For this case, $|\varepsilon|$ was less than $0.20 \%$. Corresponding results for the effective plate are also shown in these figures by triangles. A good agreement can be seen between the results for the effective plate and 35-layer plate. Figure 4.8 shows that $\left|R_{11}\right|$ approaches zero at normalized 
crack length near 0.74 . At this value of crack length almost all the incident energy is absorbed by the fundamental antisymmetric transmitted propagating wave. A comparison of Figures 4.6 and 4.7 with Figures 4.8 and 4.9 shows that the reflection coefficients of the symmetric incident wave behaves significantly different from those of the antisymmetric incident wave with the crack length.

An overall observation that can be made from Figures 4.3-4.9 is that the scattered signal amplitudes are quite sensitive to the crack length. Therefore, the results presented in this section are rather important for ultrasonic nondestructive evaluation of surface breaking cracks in laminated composite plates.

\subsubsection{Normal Central Crack Extending to Delamination}

Numerical results for scattering by a normal central crack that grows into delamination are presented in the current section (example 4). The frequency spectrum for this example is shown in Figure 4.10 where $\gamma=(k H) / 2$. Figures 4.11-4.16 show the variations in the magnitudes of reflection and transmission coefficients with the crack length at a normalized frequency of 4.0. It can be seen from the frequency spectrum that, at this frequency, there are three symmetric and three antisymmetric propagating modes.

Figures $4.11,4.12$, and 4.13 correspond to the first, second, and third symmetric incident modes, respectively. The maximum value of $|\varepsilon|$ for the results presented in these figures was $0.20 \%$. It is seen from Figure 4.11 that $\left|T_{1 n}\right|$ and $\left|R_{1 n}\right|(n=1,2,3)$ are almost not sensitive to the extent of delamination. Figure 4.12 shows that $\left|T_{22}\right|$ and $\left|R_{22}\right|$ are the most sensitive to the crack length and $\left|T_{23}\right|$ and $\left|R_{23}\right|$ are the least affected by the extent of the crack. $\left|T_{2 n}\right|$ and $\left|R_{2 n}\right|(n=1,2)$ change gradually until crack is about half way through the thickness of the middle lamina. Then $\left|T_{22}\right|$ and 
$\left|R_{22}\right|$ change quite rapidly as the crack is almost all the way through the middle $90^{\circ}$ lamina. As the crack grows into delamination at the interfaces between the middle $\left(90^{\circ}\right)$ and adjacent lamina $\left(0^{\circ}\right)$, these coefficients change less rapidly in a linear manner. $\left|T_{21}\right|$ and $\left|R_{21}\right|$, on the otherhand, tend to decrease reaching a plateau as the delaminations become $b_{1}$ in length. It can be noticed from Figure 4.13 that $\left|T_{3 n}\right|$ and $\left|R_{3 n}\right|(n=1,2,3)$ are not affected much by the crack. A comparison between Figure 4.11 and 4.12 shows that the behaviour of $\left|T_{12}\right|$ and $\left|R_{12}\right|$ is very similar to $\left|T_{21}\right|$ and $\left|R_{21}\right|$. This is expected from the reciprocity relations between the pairs $\left(T_{p n}, T_{n p}\right)$ and $\left(R_{p n}, R_{n p}\right)$ established in $\S 4.4 .5$.

Figures $4.14,4.15$, and 4.16 correspond to first, second, and third antisymmetric incident modes, respectively. For the range of the crack lengths considered in these figures, $|\varepsilon|$ was less than $0.17 \%$. One can notice from these three figures that, at the frequency considered here, the antisymmetric incident modes are not very sensitive to the normal central crack even when the crack was almost all the way through the thickness of the middle $90^{\circ}$ lamina. However, as the crack grows into delamination, $T_{1 n}$ and $R_{1 n}(n=1,2,3)$ change rapidly with the extent of the delamination. The coefficients $\left|T_{11}\right|$ and $\left|R_{11}\right|$ show the most sensitivity to the increasing length of delamination as seen from Figure 4.14.

Numerical results of the magnitudes of transmission and reflection coefficients as a function of frequency for example 4 are presented in Figures 4.17-4.24. For the results presented in these figures $|\varepsilon|$ was less than $0.21 \%$. Figures $4.17-4.20$ correspond to first symmetric incident mode; Figures 4.21 and 4.22 correspond to second symmetric incident mode; and Figures 4.23 and 4.24 are for third symmetric incident mode. For the purpose of presentation, two crack lengths have been chosen. Figures $4.17,4.19,4.21$, and 4.23 are for the case where normal central crack has grown all the way through the thickness of the middle $90^{\circ}$ lamina viz. when $b / b_{1}=$ 
1.0. Results when the delaminations have become $b_{1}$ in length, namely when $b / b_{1}=$ 1.5, are shown in Figures 4.18, 4.20, 4.22, and 4.24.

It can be seen from Figure 4.17 that for the normal central crack, $\left|T_{11}\right|$ and $\left|R_{11}\right|$ are almost unaffected by the frequency until $\Omega$ is about 3.0. Then these coefficients change gradually as $\Omega$ increases from 3.0 to 4.0 . On the other hand, for the delamination, as seen in Figure 4.18, $\left|T_{11}\right|$ and $\left|R_{11}\right|$ change quite abruptly in the normalized frequency range $1.6-2.3$. This suggests that ultrasonic nondestructive evaluation in this frequency range will make it possible to decide whether the normal central crack has grown into delamination. Furthermore, Figure 4.18 shows that $\left|T_{11}\right|$ and $\left|R_{11}\right|$ for the delamination are not very sensitive to the frequency as $\Omega$ increases from 2.3 to 4.0 . It is seen from Figure 4.19 that $\left|T_{13}\right|$ and $\left|R_{13}\right|$ do not change appreciably with frequency for the normal central crack. However, $\left|T_{12}\right|$ and $\left|R_{12}\right|$ increases gradually in the normalized frequency range $2.6-4.0$. A similar trend in $\left|T_{1 n}\right|$ and $\left|R_{1 n}\right|(n=2,3)$ for the delamination can be seen from Figure 4.20. A comparison between Figures 4.19 and 4.20 shows that $\left|T_{1 n}\right|$ and $\left|R_{1 n}\right|(n=2,3)$ are not sensitive to the crack length in the frequency range considered. It can be noticed from Figures 4.21 and 4.22 that the most frequency sensitive coefficients of second symmetric incident mode are $\left|T_{22}\right|$ and $\left|R_{22}\right|$ in the normalized frequency range $3.0-4.0$. Also it is seen that $\left|T_{13}\right|$ and $\left|R_{13}\right|$ are the least sensitive. Moreover, a comparison between Figures 4.21 and 4.22 reveals that $\left|T_{22}\right|$ and $\left|R_{22}\right|$ for the normal crack $\left(b / b_{1}=1.0\right)$ and the delamination crack $\left(b / b_{1}=1.5\right)$ are quite different in the normalized frequency range $3.0-4.0$. Figures 4.23 and 4.24 show that $\left|T_{3 n}\right|$ and $\left|R_{3 n}\right|(n=1,2,3)$ are not very much affected by the frequency. However, among the three scattered propagating modes of third symmetric incident mode, second mode is the most sensitive one to the frequency and the crack length. 


\subsubsection{General Case}

To illustrate the general case, some numerical results for scattering by a symmetric normal edge crack are presented in this section. Note that since the impedance matrix, $[S]$, (refer to equation $4.19 \mathrm{~b}$ ) for the general case is complex, and each node involves three degrees of freedom, the core storage requirement for the impedance matrix is approximately 4.5 times that required for plane strain case with the same finite element mesh.

Numerical results for the magnitudes of transmission and reflection coefficients $\left(\left|T_{p n}\right|\right.$ and $\left.\left|R_{p n}\right|\right)$, and proportions of transmitted and reflected energies $\left(E_{p n}^{-}\right.$and $E_{p n}^{+}$) for scattering by a symmetric normal edge crack in a homogeneous graphite epoxy plate (example 2) are presented in Table 4.1. The percentage error in energy balance, $\varepsilon$, is presented also in this table. The normalized frequency considered is 2.0. At this frequency, when wave propagation occurs in an uncracked plate in a direction making an angle $45^{\circ}$ with the fiber direction, it is found that the dispersion equation for the off-axis propagation case (described in $§ 2.3 .3$ ) has two real roots corresponding to symmetric modes. The fundamental or the first root (the one which has the largest magnitude among the real roots) has been considered here. The numerical value of this root is $2.0371\left(=k^{(o)}\right)$. Part (a) of Table 4.1 corresponds to $\theta=0^{\circ}$ and $\phi^{\text {in }}=45^{\circ}$. Thus, $\zeta_{0}^{\prime}$ is equal to $1.4404\left(=2.0371 \sin 45^{\circ}\right)$. For this value of $\zeta_{0}$, the dispersion equation for general case (see equation 4.45 ) has two real roots for wavenumber $k$. These two roots are listed in the second column of part (a) of Table 4.1. Part (b) of this table is for $\theta=22.5^{\circ}$ and $\phi^{\text {in }}=22.5^{\circ}$, thus resulting in $\zeta_{0}$ of $0.7796\left(=2.0371 \sin 22.5^{\circ}\right)$. Corresponding wave numbers of propagating modes obtained as roots from the dispersion equation for general case are reported in the second column of part (b) of Table 4.1. It is seen from Table 4.1 that the energy balance and the reciprocity between the pairs $\left(E_{p n}^{-}, E_{n p}^{-}\right)$is satisfied with 
negligible errors. Part (a) of Table 4.1 shows that the reciprocity between the pair $\left(E_{p n}^{+}, E_{n p}^{+}\right)$is satisfied with negligible errors when the fibers are aligned in the $x$ direction. This is expected from equation (D.35) in Appendix D. It is seen also that the reflection coefficients are sensitive to the crack length. Note that in both part (a) and (b) of Table 4.1, for the first symmetric incident mode $(p=1)$, the incident wave normal is in a direction making $45^{\circ}$ with the fiber direction. Thus, for the first symmetric mode, the results of part (b) can be thought of as those corresponding to a crack which is oriented (in $x y$ plane) in a $22.5^{\circ}$ direction to the orientation of the crack in part (a). In view of this, a comparison between (a) and (b) parts of Table 4.1 discloses that the reflection and transmission coefficients are quite sensitive to the orientation of the crack.

Finally, Table 4.2 shows the numerical results for scattering by a symmetric normal edge crack in an 8-layer $0^{\circ} / 90^{\circ} / 0^{\circ} / 90^{\circ} / 90^{\circ} / 0^{\circ} / 90^{\circ} / 0^{\circ}$ graphite-epoxy plate (example 5). The results presented correspond to a normalized frequency of 4.0 and a normalized crack length of 0.5 . For wave propagation in the uncracked plate in a $45^{\circ}$ direction to the $0^{\circ}$ fibers, off-axis dispersion equation gave the wave number $\left(k^{(o)}\right)$ of the fundamental symmetric propagating mode as 4.7551 . This results in a $\zeta_{0}$ of 3.3624 for part (a) and 1.8197 for part (b) of Table 4.2. For these $\zeta_{0}$ values, at a normalized frequency of 4.0 , the general case dispersion equation leads to two real roots for part (a) and three real roots for part (b), all of which correspond to real symmetric propogating modes. These wave numbers $\left(k_{p}\right)$ are listed in the second column of Table 4.2. It can be seen from this table that the energy balance and the reciprocity relations among proportions of energy are satisfied with small errors. Numerical results for $\left|T_{11}\right|$ and $\left|R_{11}\right|$ show that these coefficients are very sensitive to the orientation of the crack (in $x y$ plane). 


\subsection{Concluding Remarks}

A hybrid method combining the finite element method with wave function expansion procedure has been presented to study time harmonic wave scattering by cracks and delaminations in laminated composite plates. The validity of the results obtained from the hybrid method is established by checking the energy balance and the satisfaction of elastodynamic reciprocity relations. It is shown that results presented here agree well with other reported results for homogeneous isotropic plate.

It is found that the reflection and transmission coefficients are very sensitive to the extent and the orientation of the crack, the incident mode number, and the frequency. The study shows that judicious choice of frequency and incident mode can be made to obtain optimum results in ultrasonic nondestructive evaluation of flaws. Only a few examples have been chosen to illustrate the applicability of the hybrid method. The hybrid formulation based finite element code developed here has the capability to investigate scattering of ultrasonic elastic waves by flaws over a wide range of the parameters involved. This enables one to perform a detailed parametric study of the scattering problem and the results of this study may be used to interpret ultrasonic test measurements to characterize flaws.

For the sake of simplicity, wave function expansion has been considered for laminated plates having orthotropic or transversely isotropic material properties. Since wave functions are available for a homogeneous plate possessing general anisotropic material properties (Nayfeh and Chimenti, 1989), by employing the propagator matrix technique, the present hybrid method can be easily adapted to laminated plates with laminates of general anisotropic material properties. 
There are several advantages of the hybrid method:

1. Each lamina of the plate can have arbitrary mechanical properties and thickness.

2. Because the scattered field is expressed in wave function expansion, an arbitrary number of layers can be accommodated without appreciable increase in computational time. This is to be contrasted with the integral representation technique using Green's function. In the latter, computational time increases considerably with increasing number of laminae because of the time involved in the computation of the Green's function.

3. The interior region containing the flaw can have quite arbitrary material properties.

4. The flaw can be quite arbitrary in geometry and in orientation. Also, the multiple scattering by a cluster of neighbouring flaws can be studied without much difficulty.

The hybrid method is suited ideally for low frequencies. At high frequencies, very fine finite element meshes are required to ensure accuracy, thus increasing the number of degrees of freedoms involved in the analysis. 


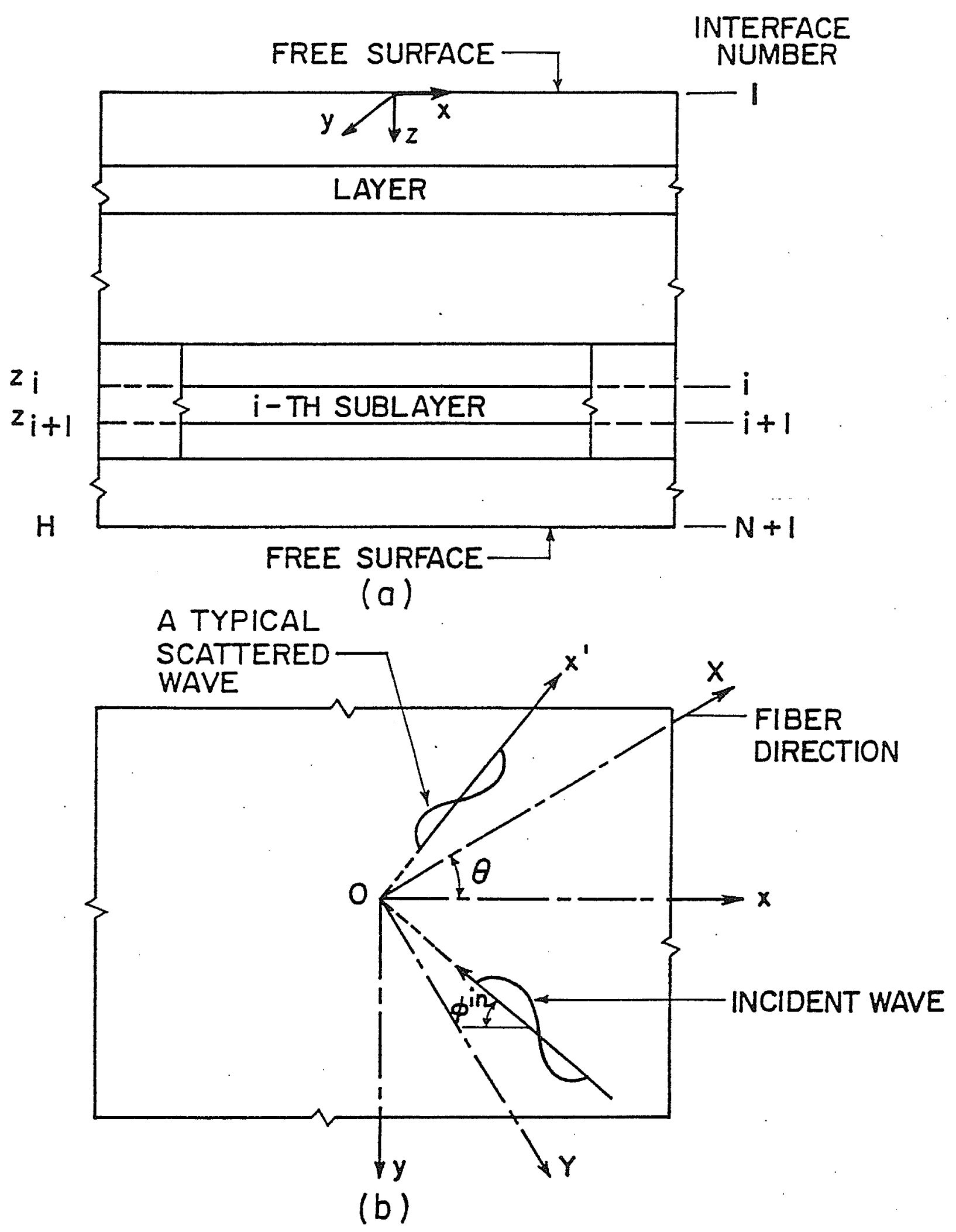

Figure 4.1: Geometry of the layered plate

(a) Elevation showing subdivision of layers,

(b) Plan view of a typical ( $i$-th) sublayer showing orientation of fibers and wave normals. 


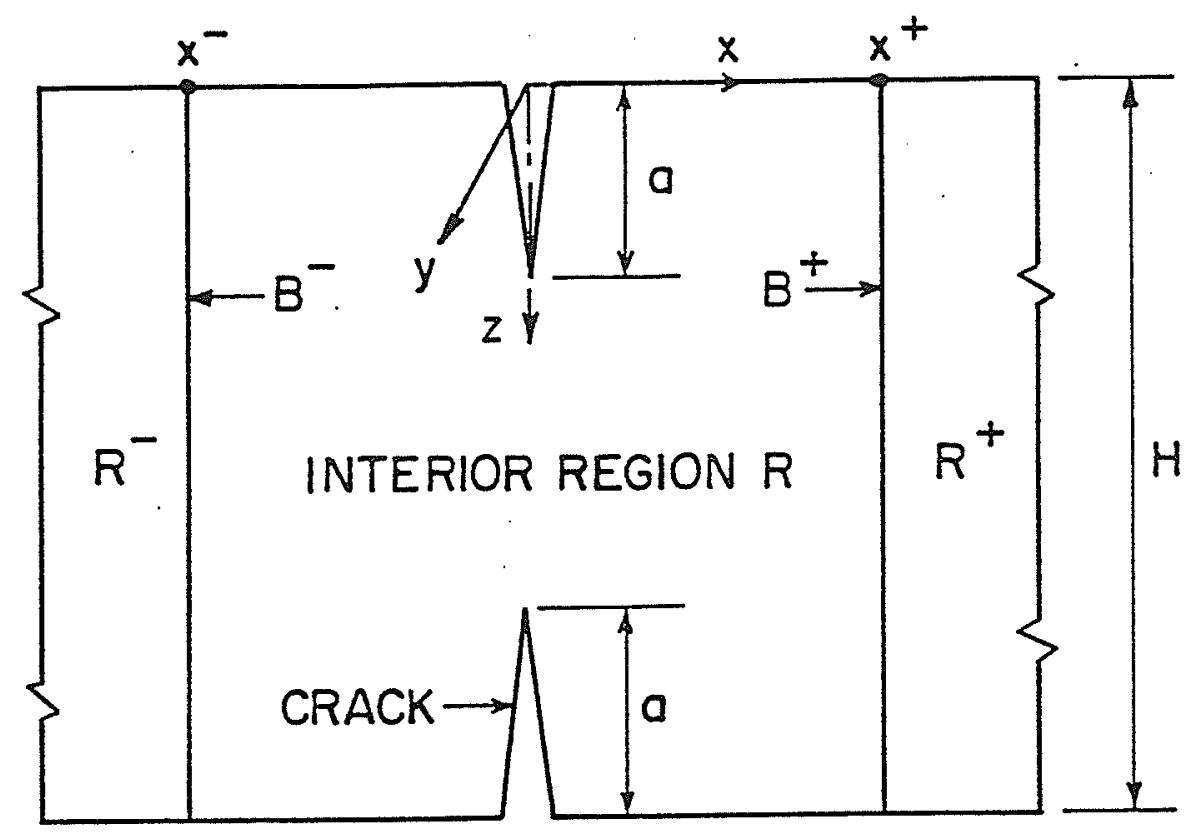

(a)

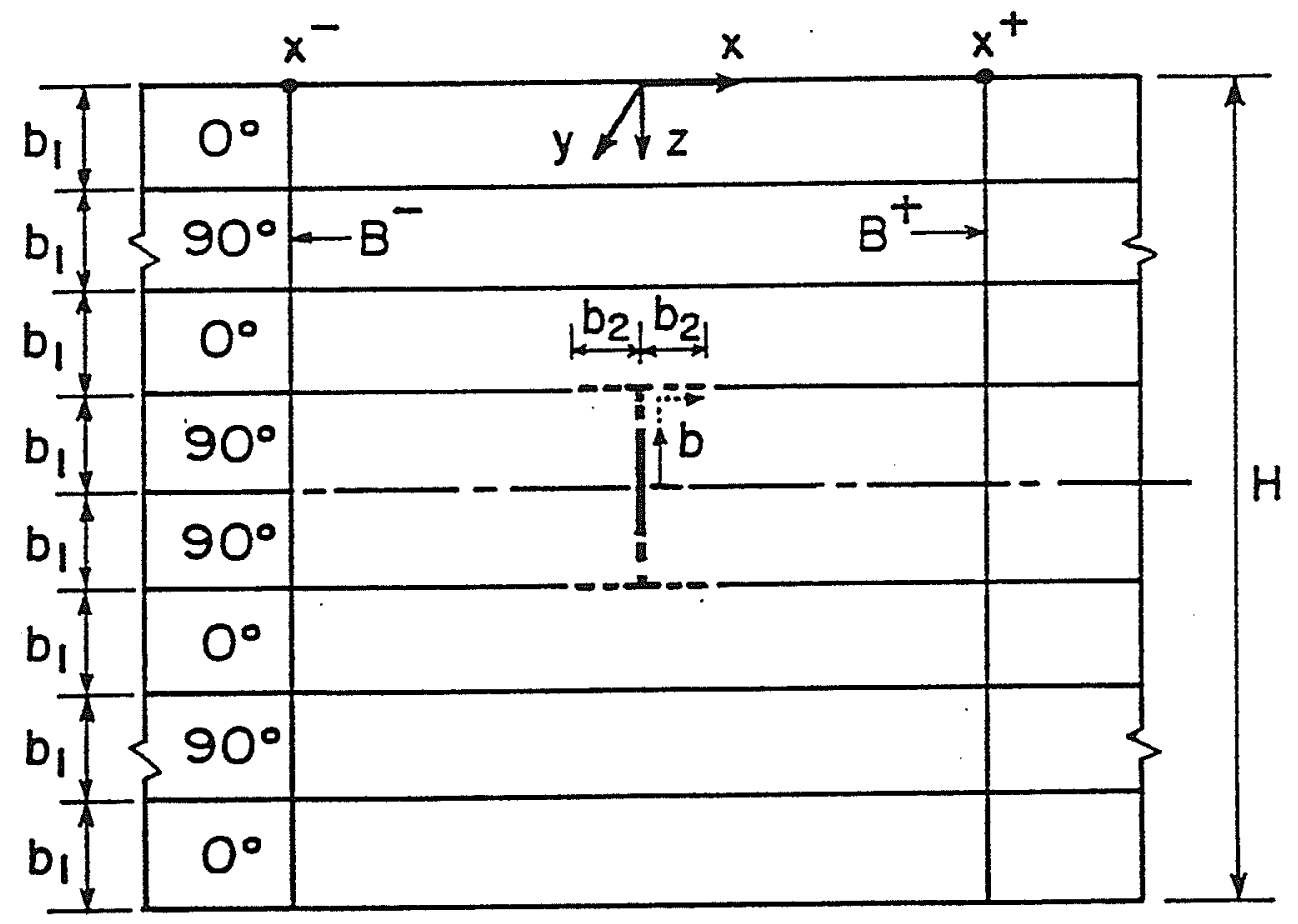

(b)

Figure 4.2 : Geometry of flaws and details of regions

(a) Normal edge crack in a plate

(b) Centrally located normal matrix crack extending to delamination (shown by solid and dashed lines) in an 8-layer cross-ply $\left(0^{\circ} / 90^{\circ} / 0^{\circ} / 90^{\circ} / 90^{\circ} / 0^{\circ} / 90^{\circ} / 0^{\circ}\right)$ plate

(c) A typical finite element mesh near the crack tip. 


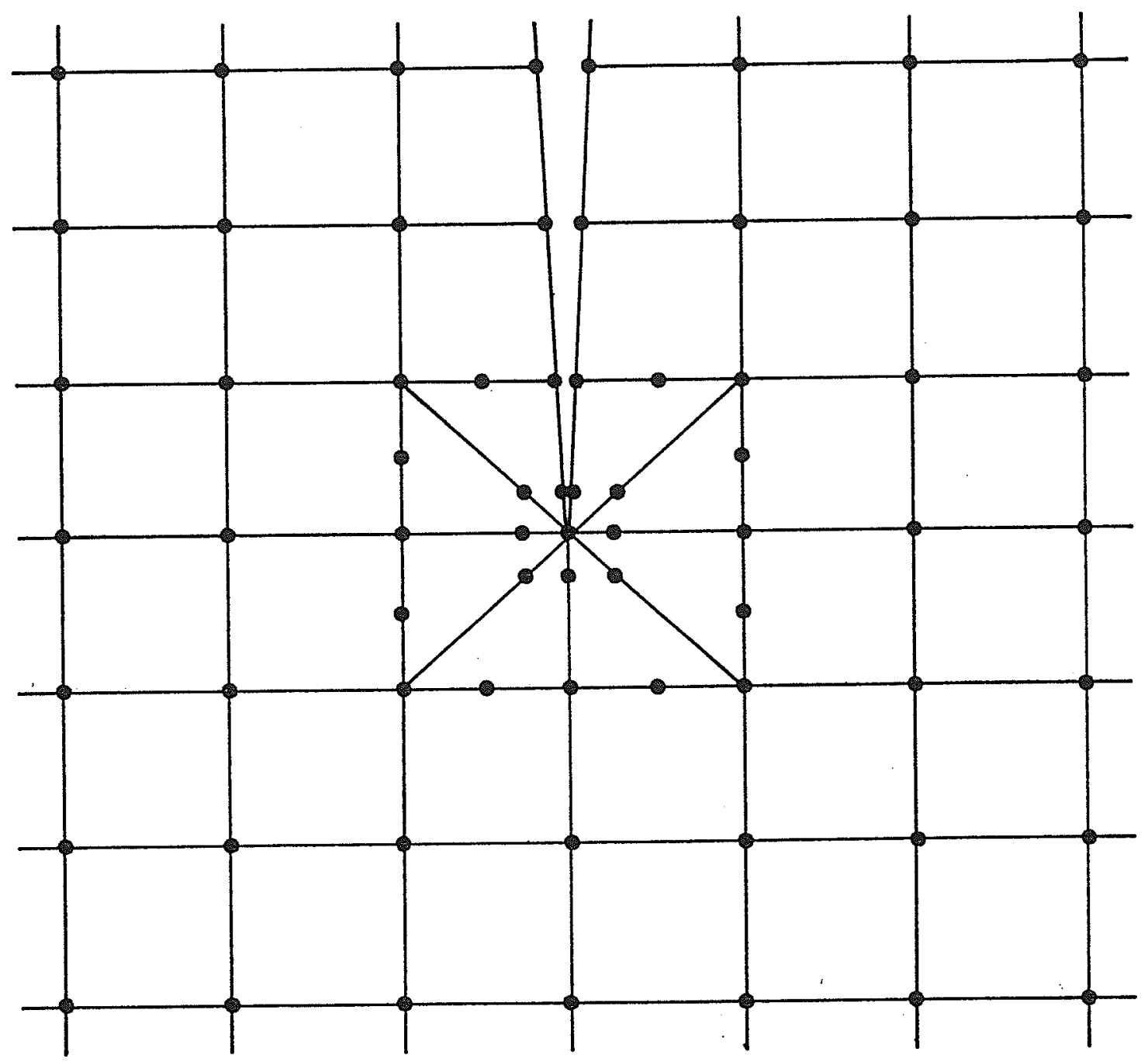

(c)

Figure 4.2 : Geometry of flaws and details of regions

(a) Normal edge crack in a plate

b) Centrally located normal matrix crack extending to delamination (shown by solid and dashed lines) in an 8-layer cross-ply

$\left.0^{\circ} / 90^{\circ} / 0^{\circ} / 90^{\circ} / 90^{\circ} / 0^{\circ} / 90^{\circ} / 0^{\circ}\right)$ plate

(c) A typical finite element mesh near the crack tip. 


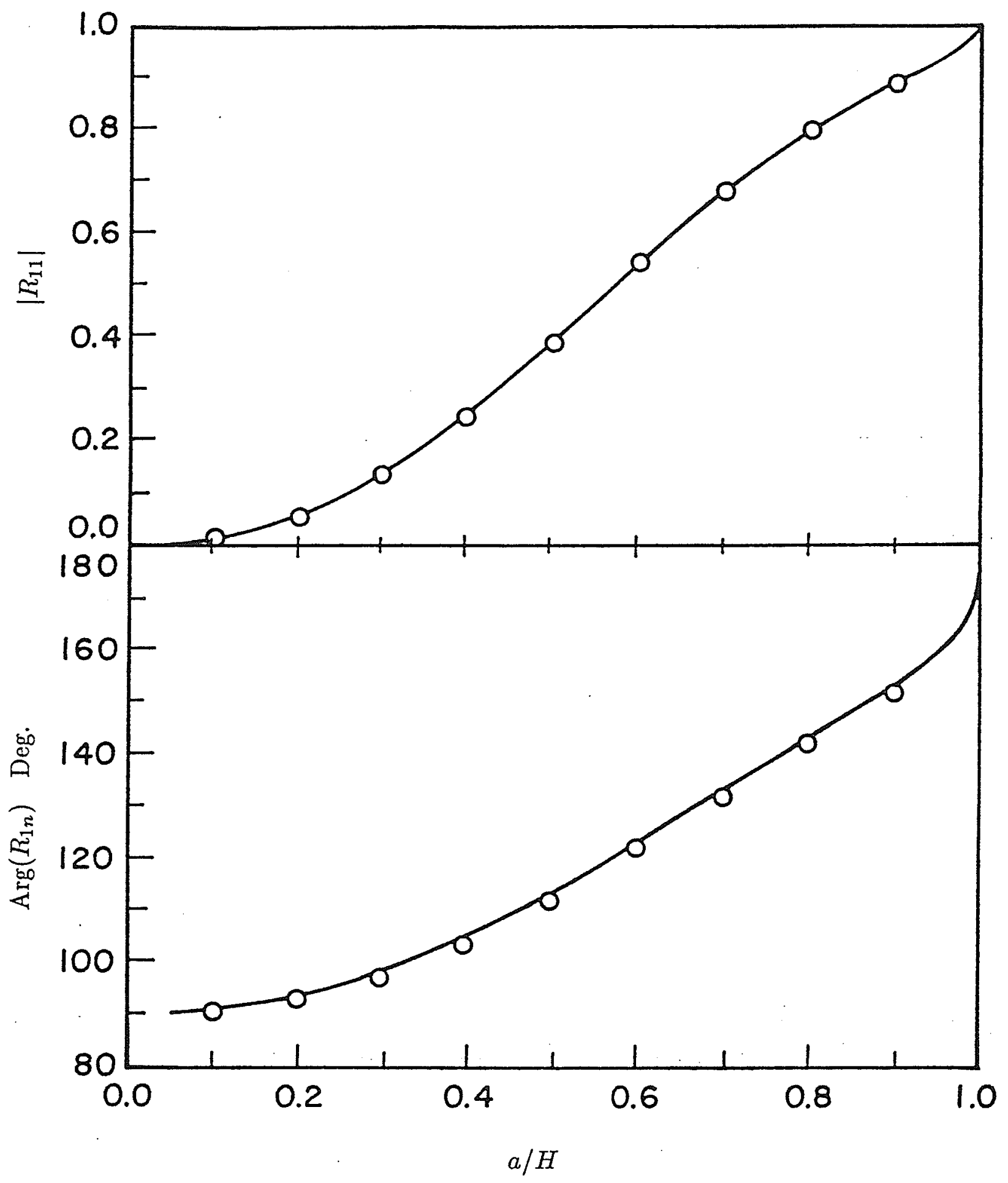

Figure 4.3: $R_{11}$ vs normalized crack length at $\Omega=\pi / 2$ for example 1 , due to first symmetric incident mode (plane strain case). 


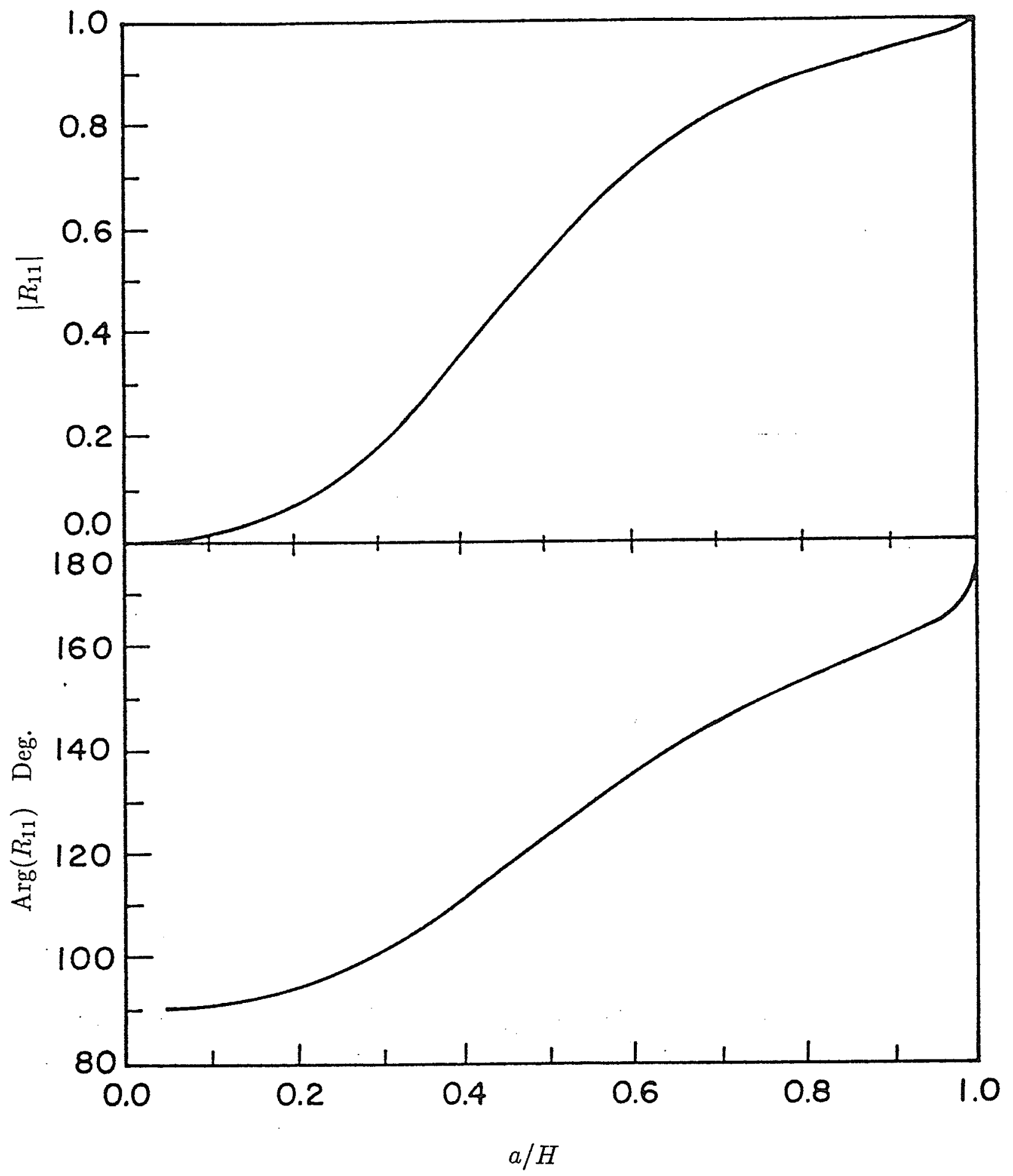

Figure 4.4: $R_{11}$ vs normalized crack length at $\Omega=2.0$ for example 2, due to first symmetric incident mode (plane strain case). 


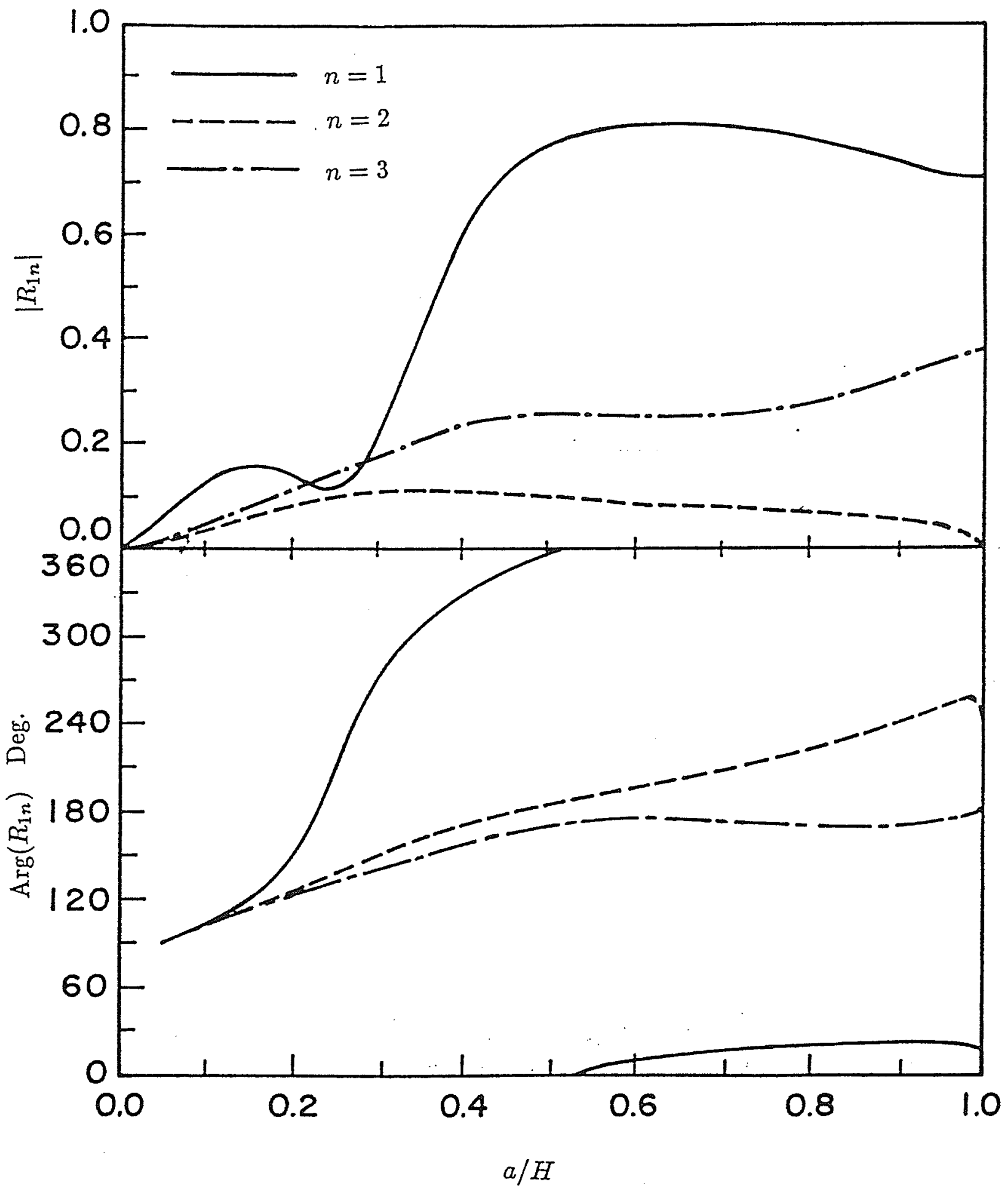

Figure $4.5: R_{1 n}$ vs normalized crack length at $\Omega=4.0$ for example 2, due to first symmetric incident mode (plane strain case). 


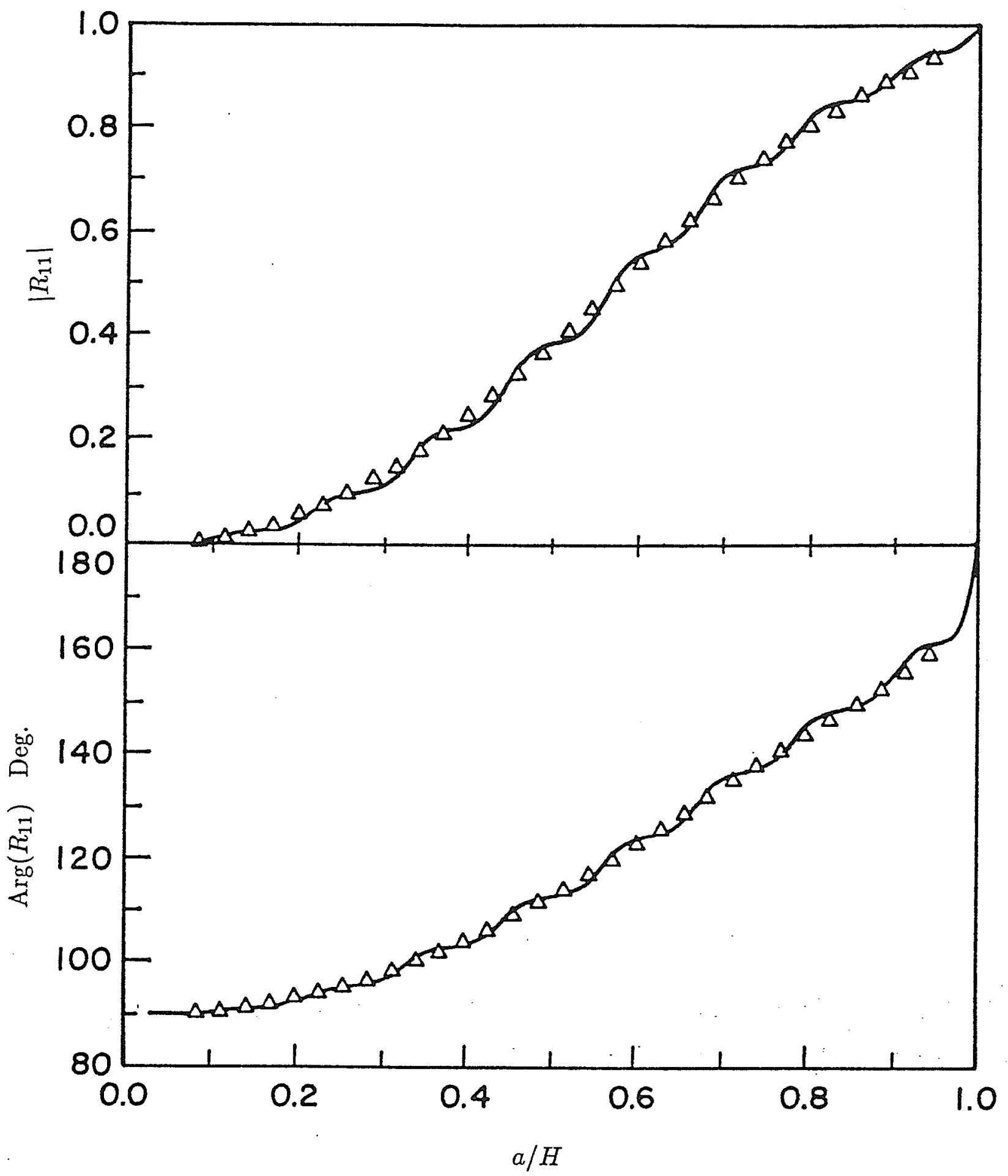

Figure 4.6: $R_{11}$ vs normalized crack length at $\Omega=1.2$ for example 3 , due to first symmetric incident mode (plane strain case). 


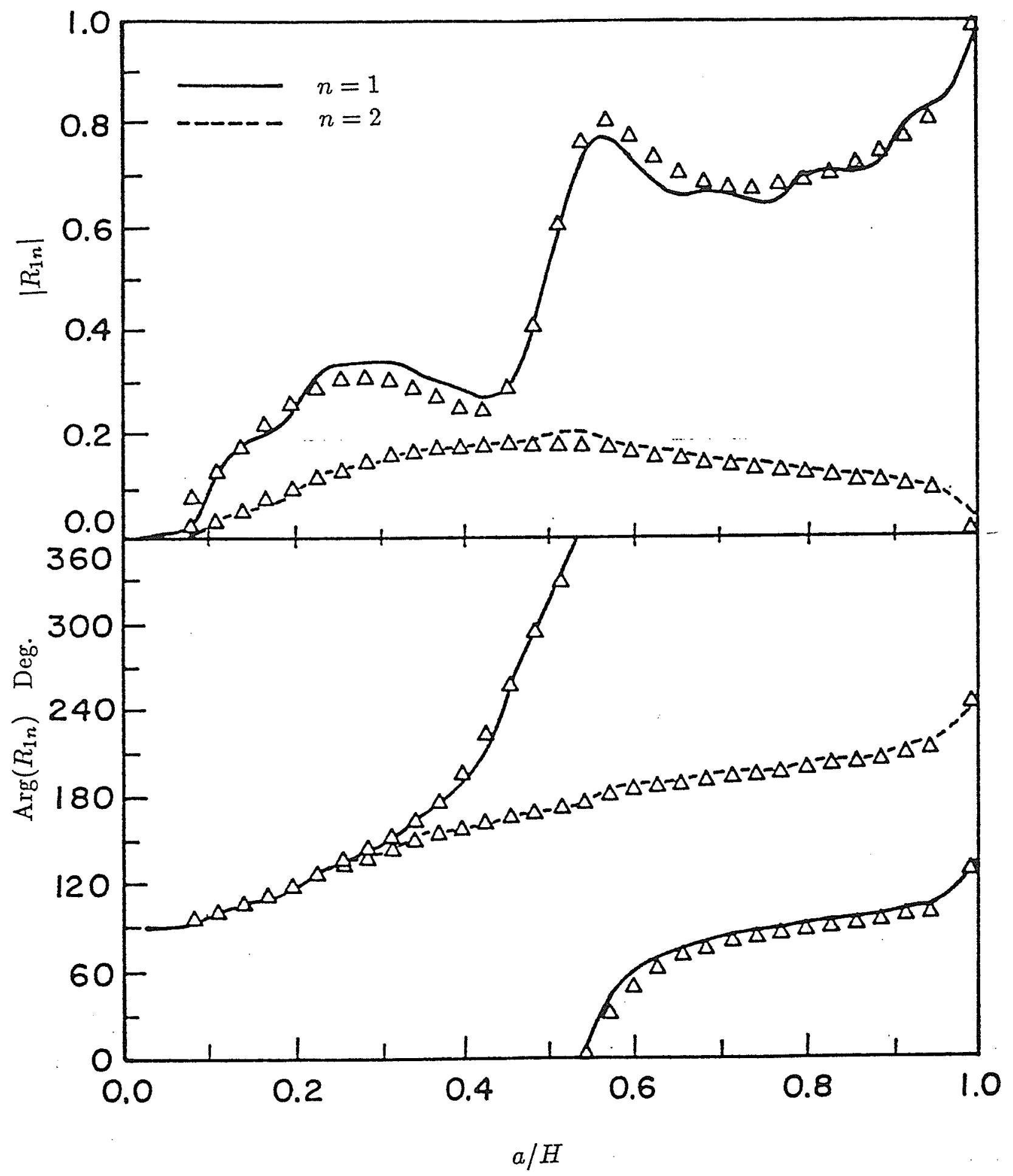

Figure $4.7: R_{1 n}$ vs normalized crack length at $\Omega=2.5$ for example 3 , due to first symmetric incident mode (plane strain case). 


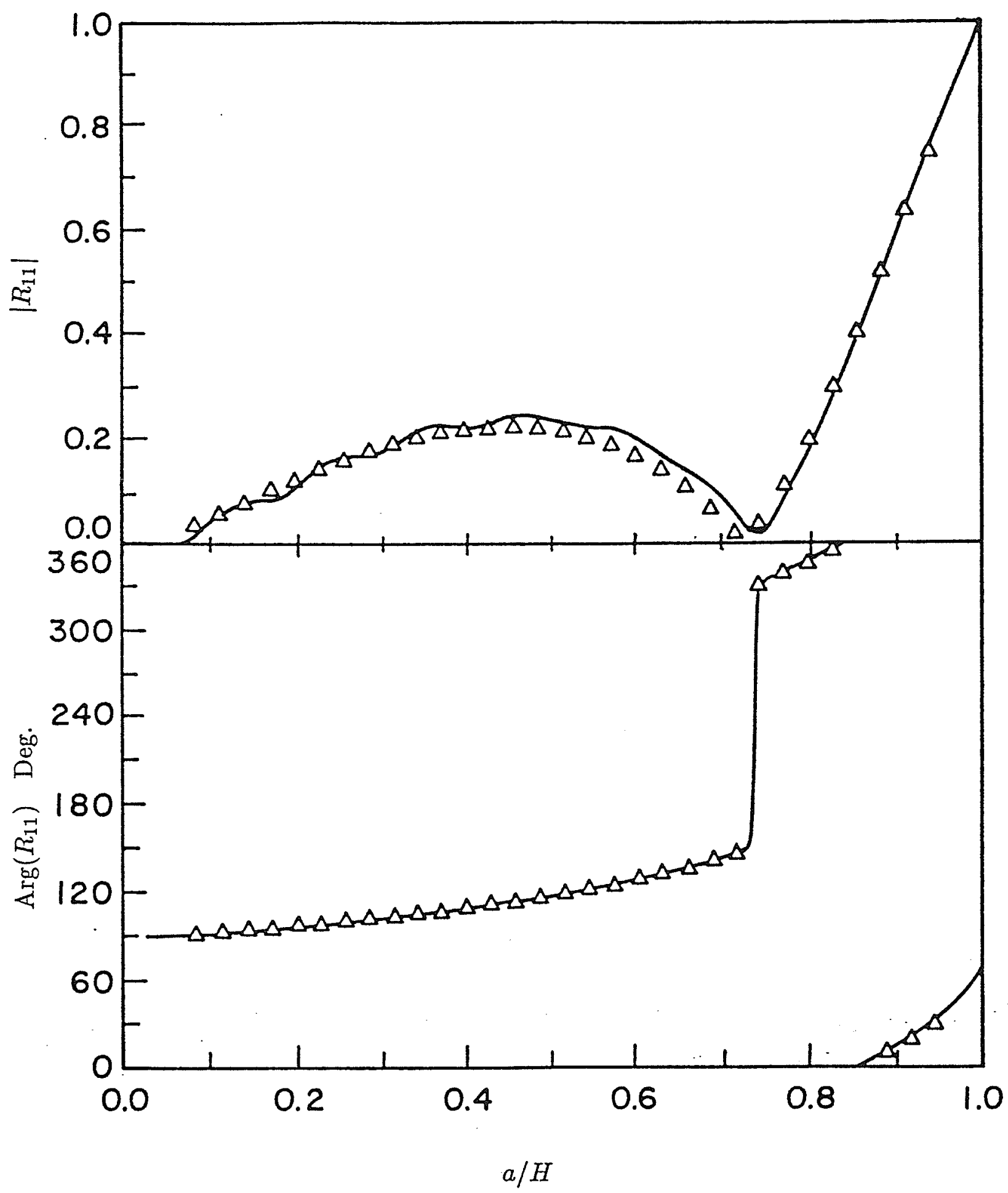

Figure 4.8: $R_{11}$ vs normalized crack length at $\Omega=1.2$ for example 3 , due to first antisymmetric incident mode (plane strain case). 


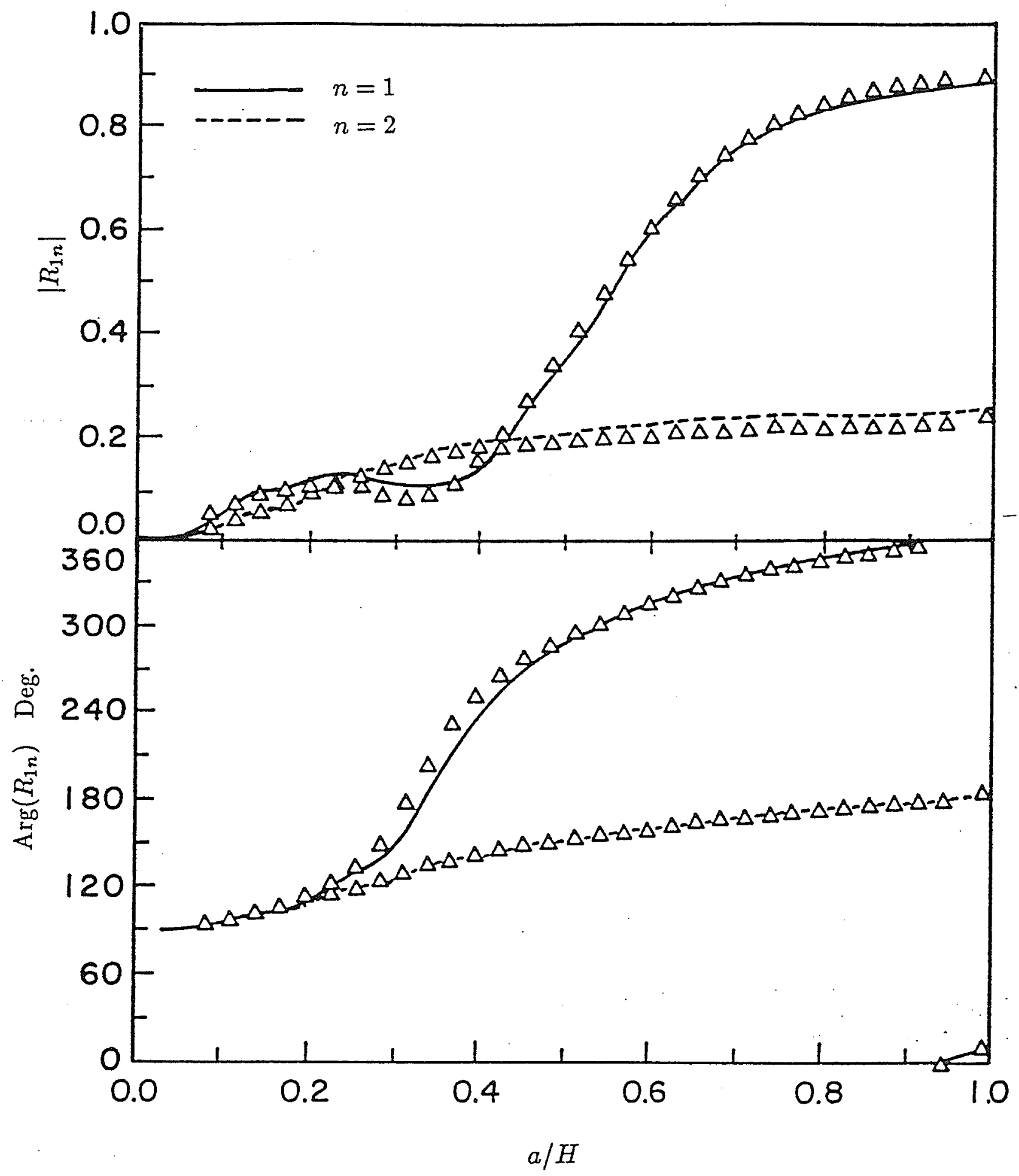

Figure $4.9: R_{1 n}$ vs normalized crack length at $\Omega=2.5$ for example 3 , due to first antisymmetric incident mode (plane strain case). 


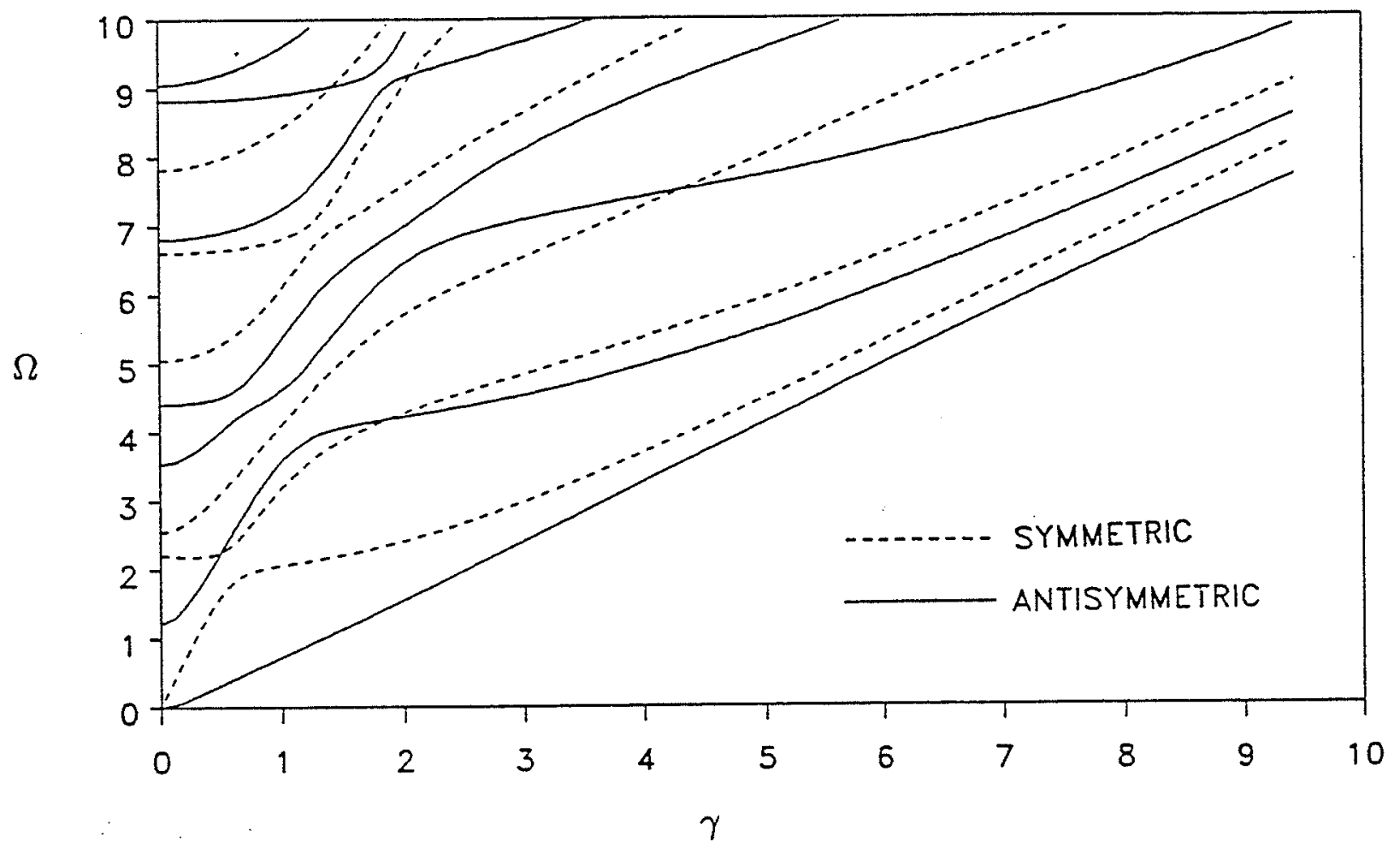

$\begin{aligned} \text { Figure 4.10: } & \text { Real branches of the frequency spectrum for an 8-layer cross-ply } \\ & \left(0^{\circ} / 90^{\circ} / 0^{\circ} / 90^{\circ} / 90^{\circ} / 0^{\circ} / 90^{\circ} / 0^{\circ}\right) \text { graphite-epoxy plate (plane strain case). }\end{aligned}$ 


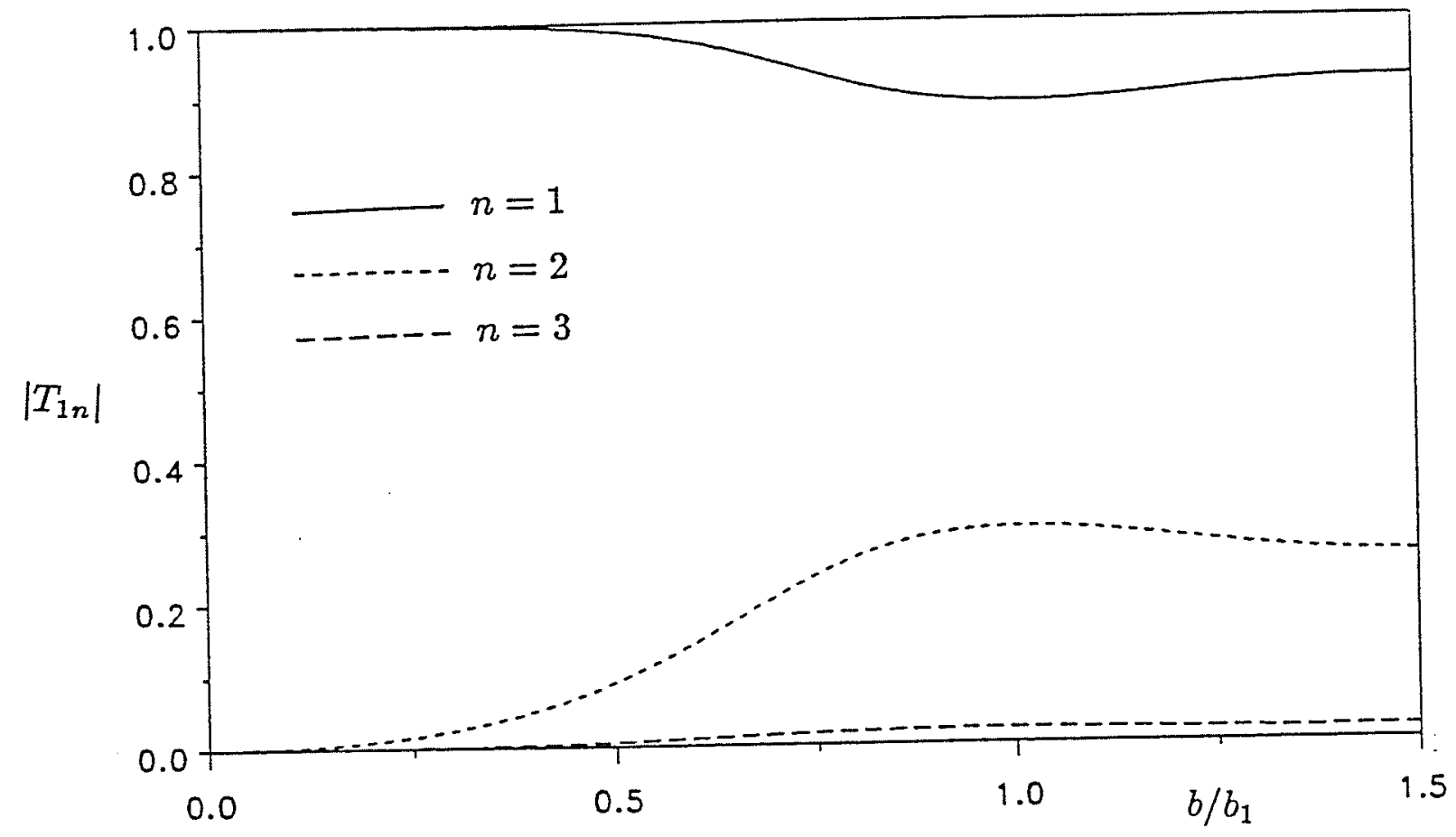

(a)

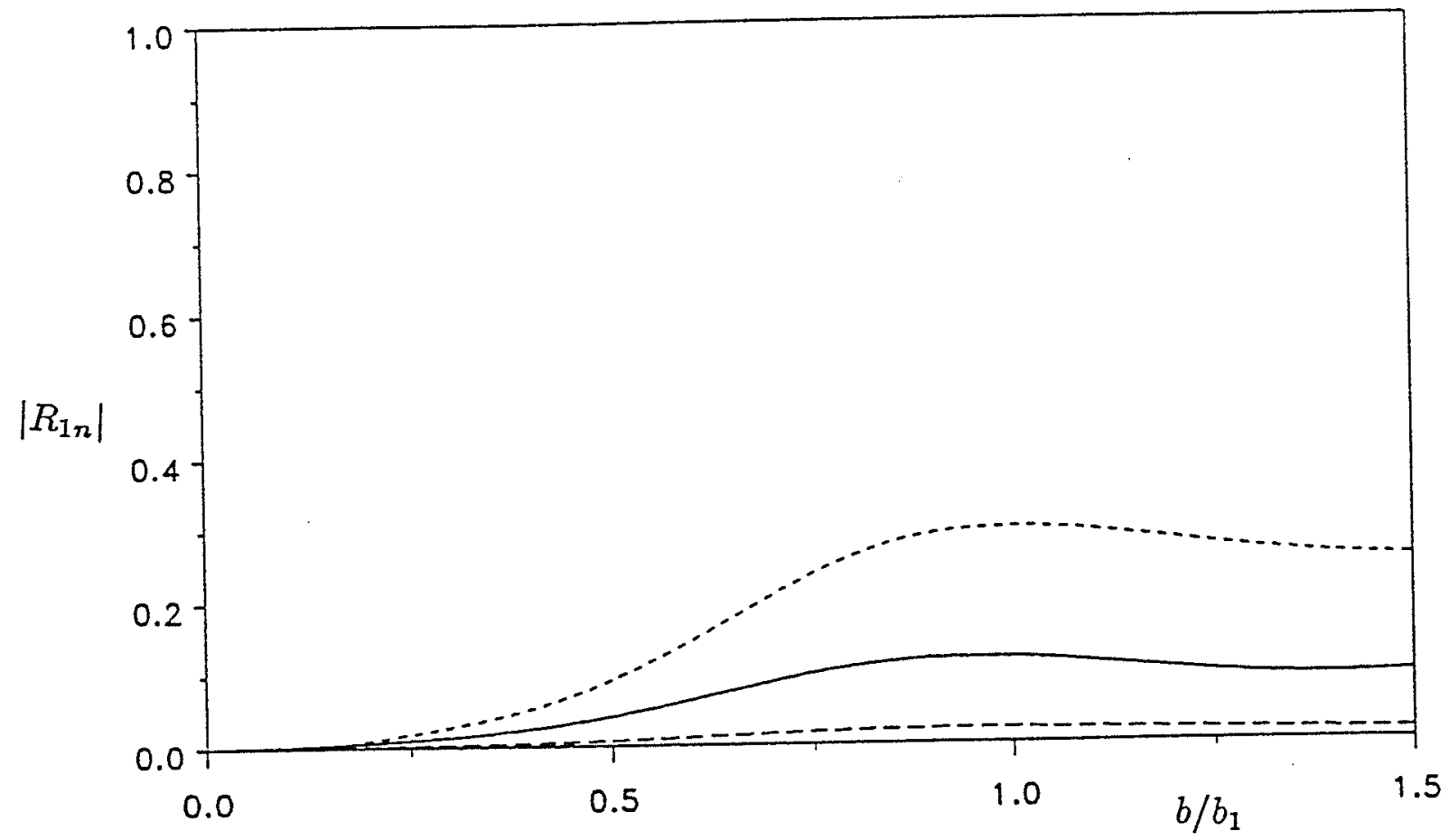

(b)

Figure 4.11 : Variation of $\left|T_{1 n}\right|$ and $\left|R_{1 n}\right|$ with normalized crack length at $\Omega=4.0$ for example 4 , due to first symmetric incident mode (plane strain case):

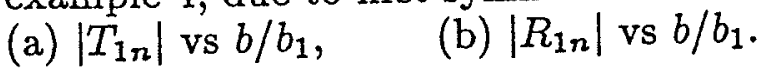




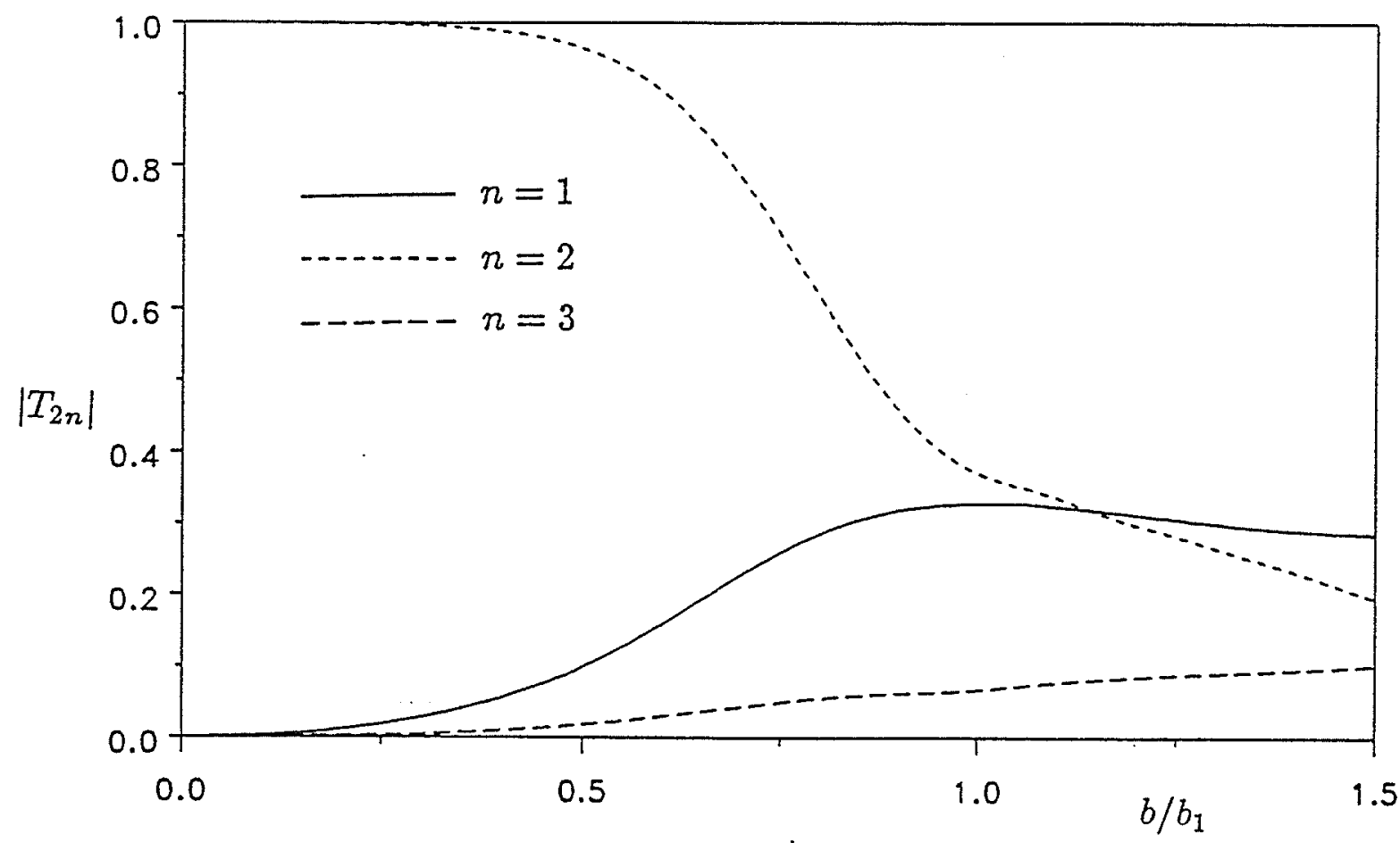

(a)

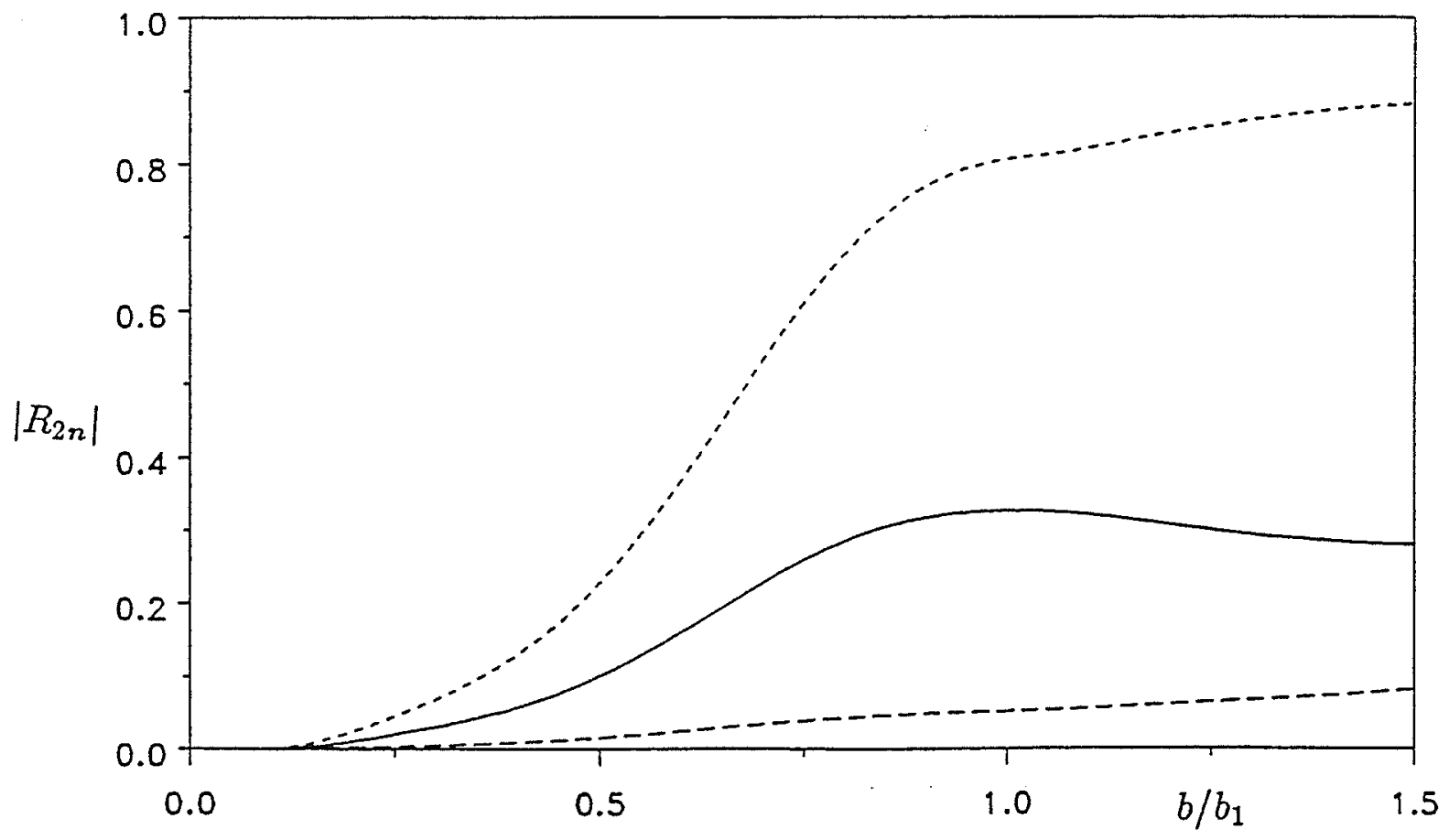

(b)

Figure 4.12: Variation of $\left|T_{2 n}\right|$ and $\left|R_{2 n}\right|$ with normalized crack length at $\Omega=4.0$ for example 4, due to second symmetric incident mode (plane strain case):
(a) $\left|T_{2 n}\right|$ vs $b / b_{1}$
(b) $\left|R_{2 n}\right|$ vs $b / b_{1}$. 


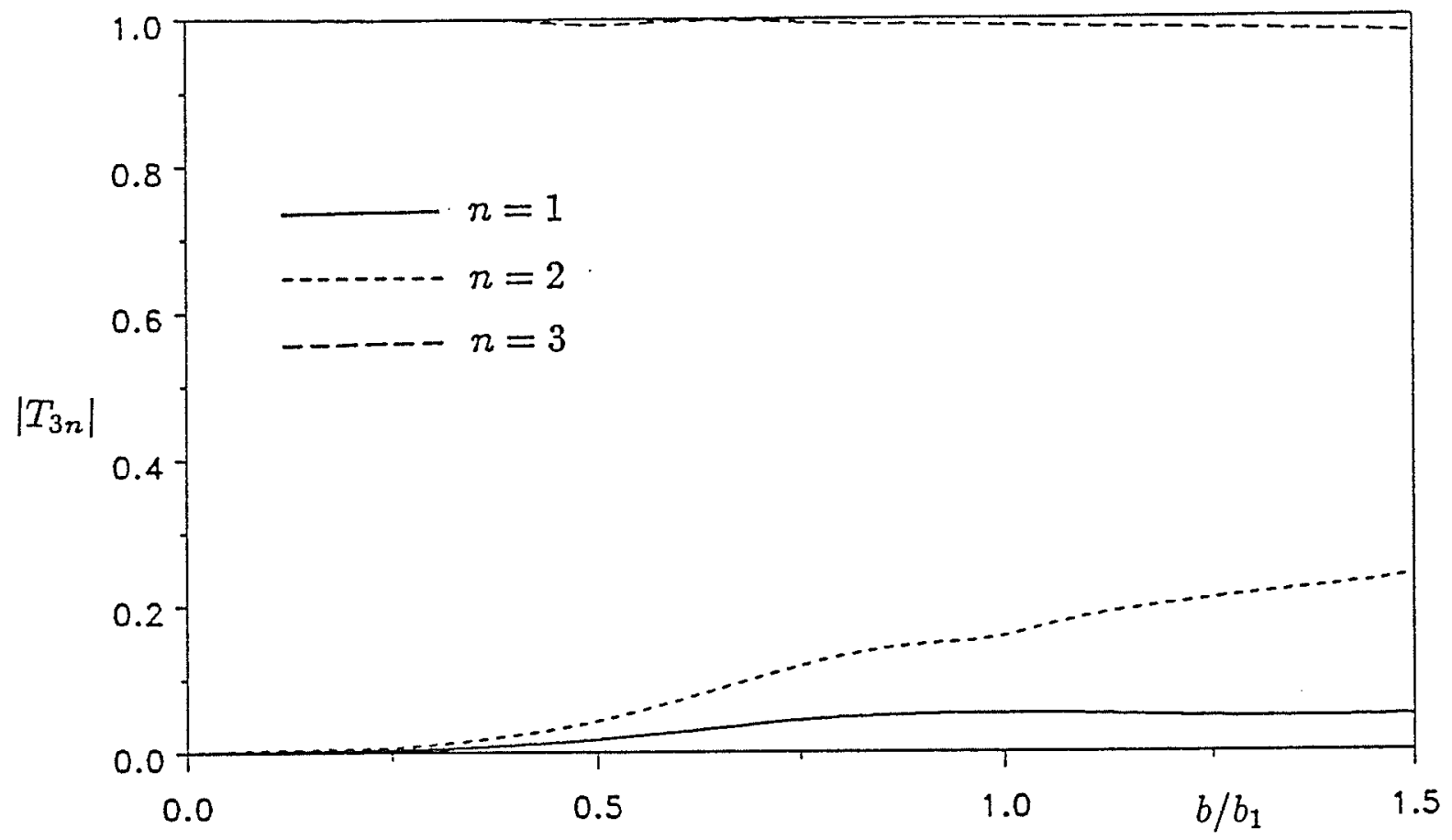

(a)

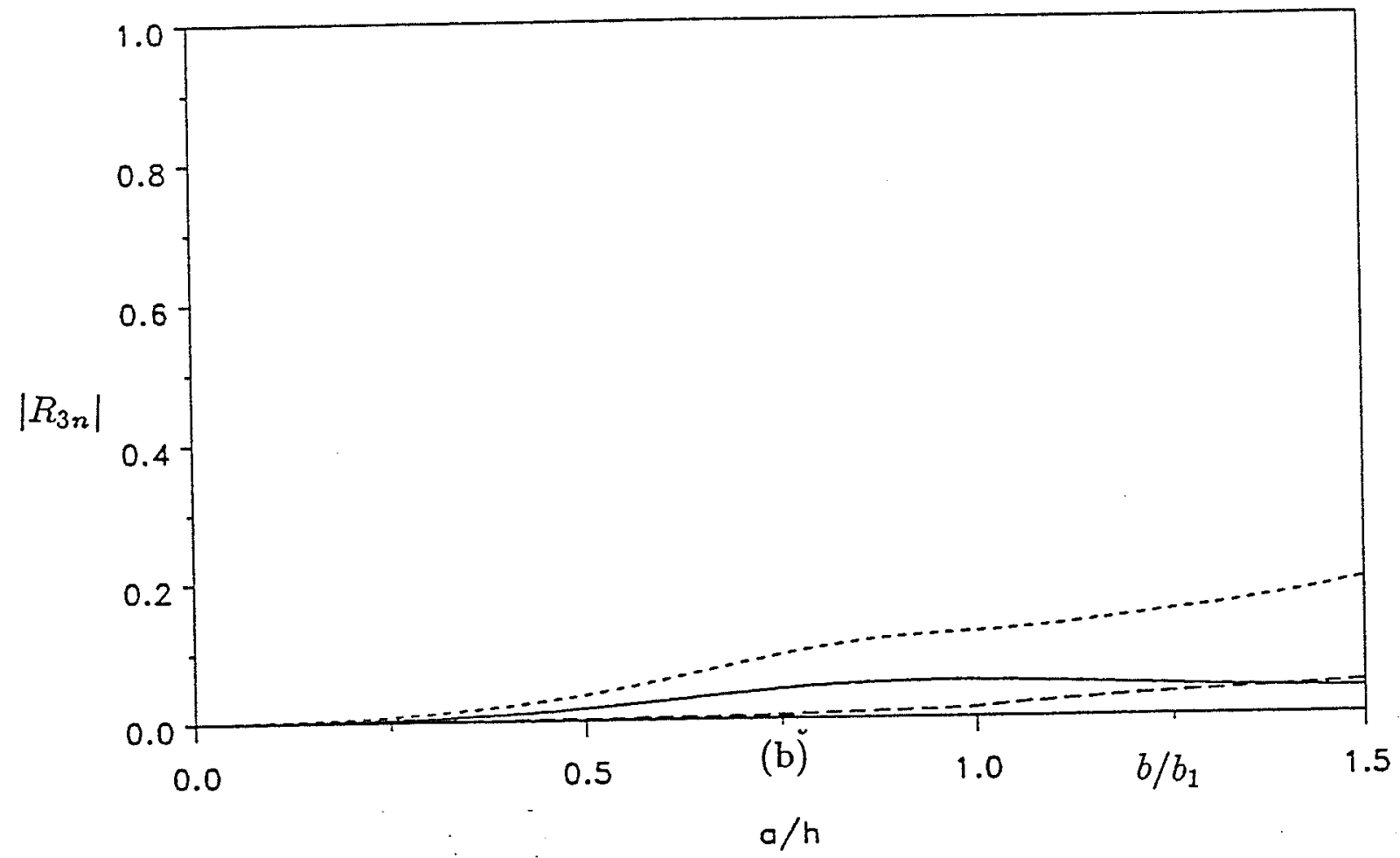

Figure 4.13 : Variation of $\left|T_{3 n}\right|$ and $\left|R_{3 n}\right|$ with normalized crack length at $\Omega=4.0$ for example 4 , due to third symmetric incident mode (plane strain case):
(a) $\left|T_{3 n}\right|$ vs $b / b_{1}$,
(b) $\left|R_{3 n}\right|$ vs $b / b_{1}$. 


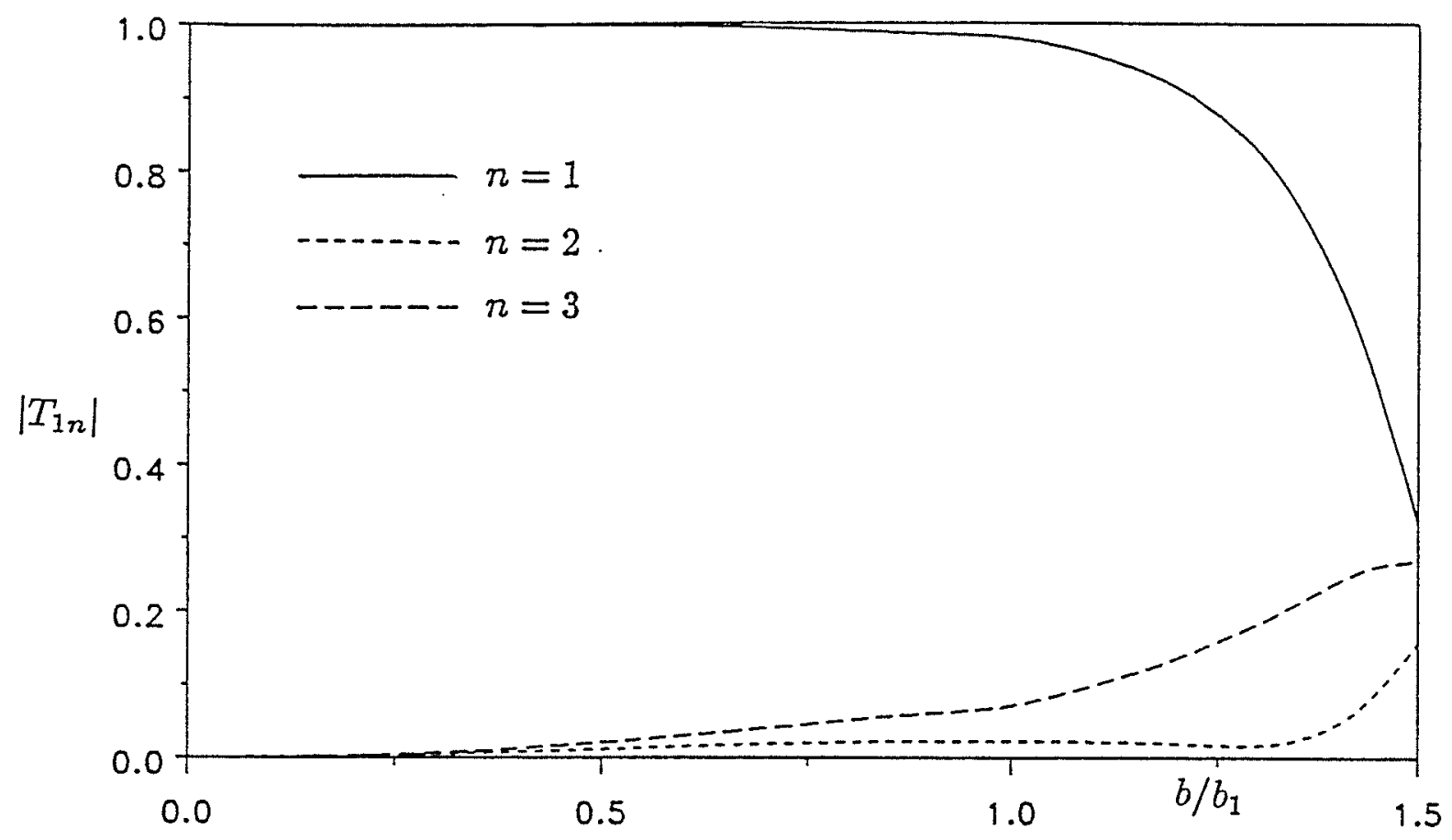

(a)

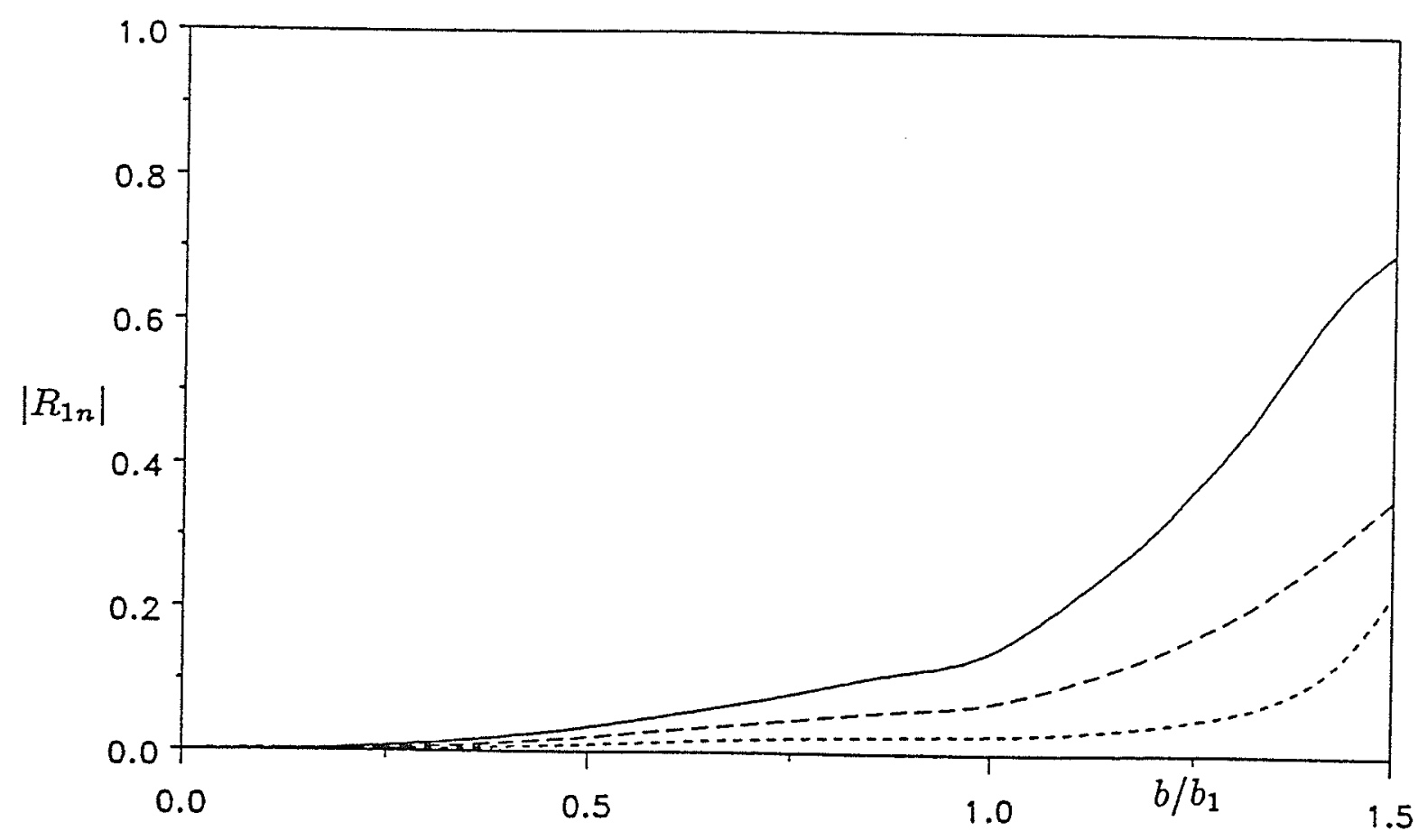

(b)

Figure 4.14: Variation of $\left|T_{1 n}\right|$ and $\left|R_{1 n}\right|$ with normalized crack length at $\Omega=4.0$ for example 4, due to first antisymmetric incident mode (plane strain case):

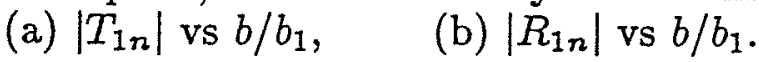




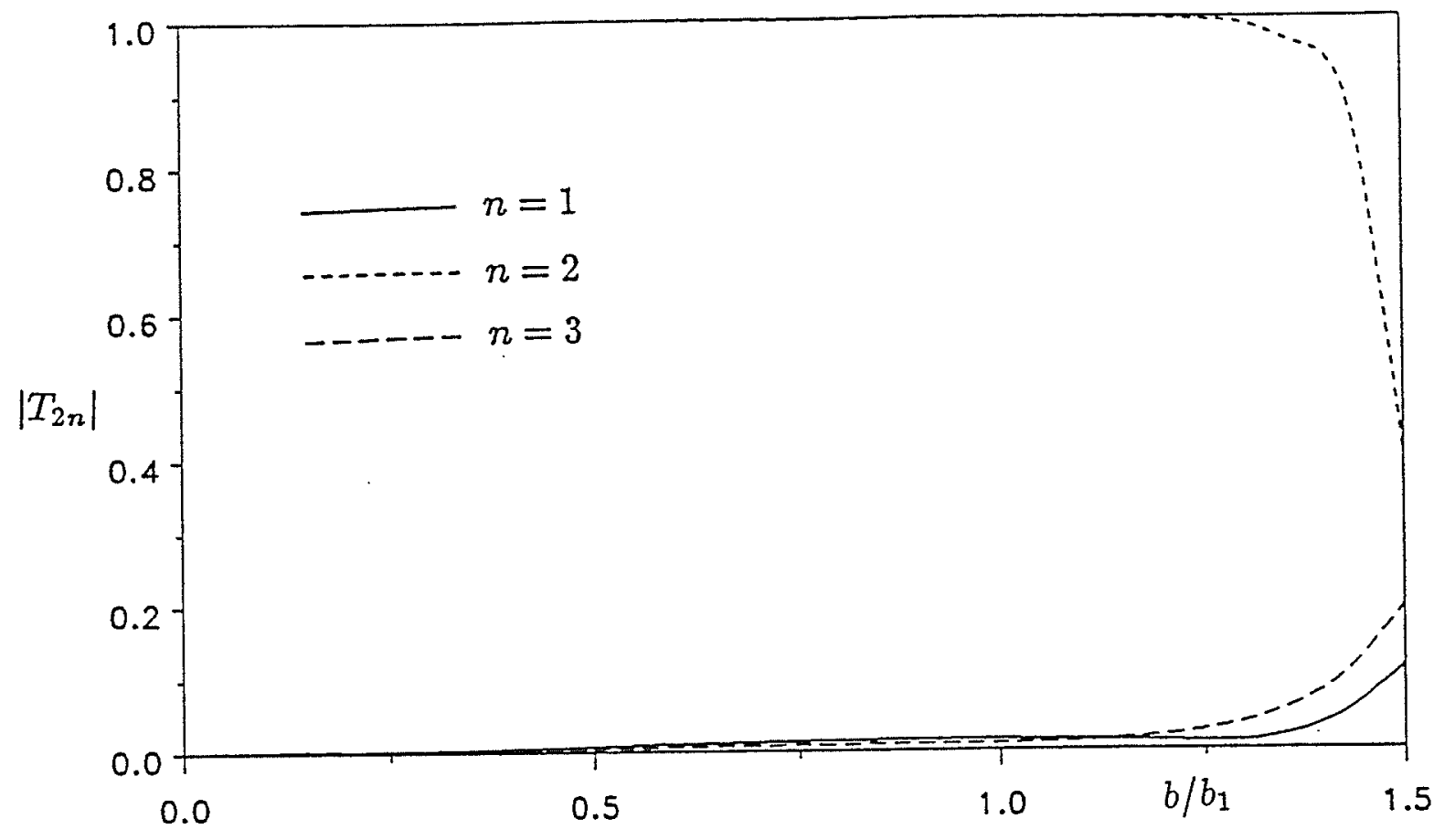

(a)

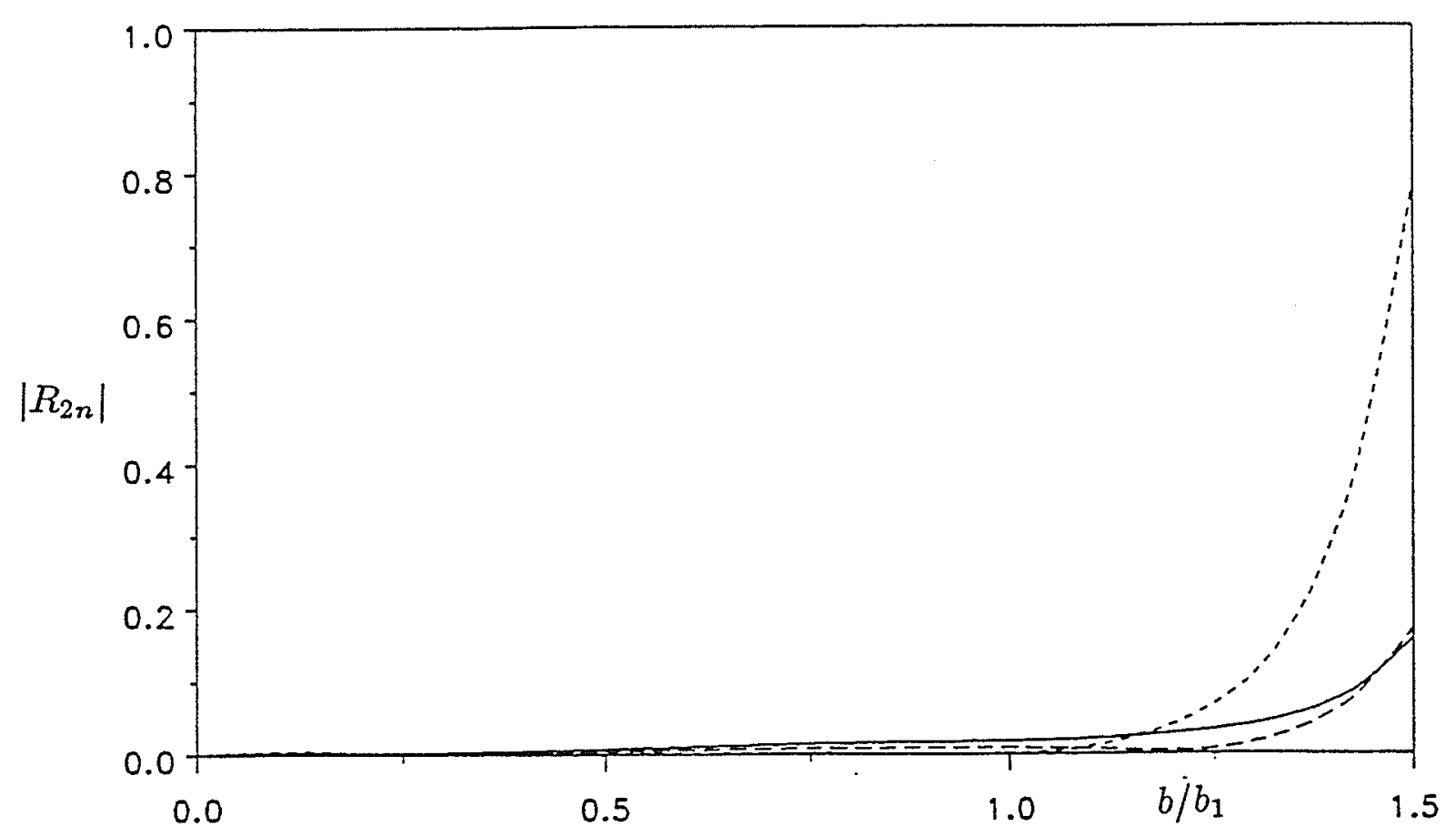

(b)

Figure 4.15: Variation of $\left|T_{2 n}\right|$ and $\left|R_{2 n}\right|$ with normalized crack length at $\Omega=4.0$ for example 4 , due to second antisymmetric incident mode (plane strain case):
(a) $\left|T_{2 n}\right|$ vs $b / b_{1}$,
(b) $\left|R_{2 n}\right|$ vs $b / b_{1}$. 


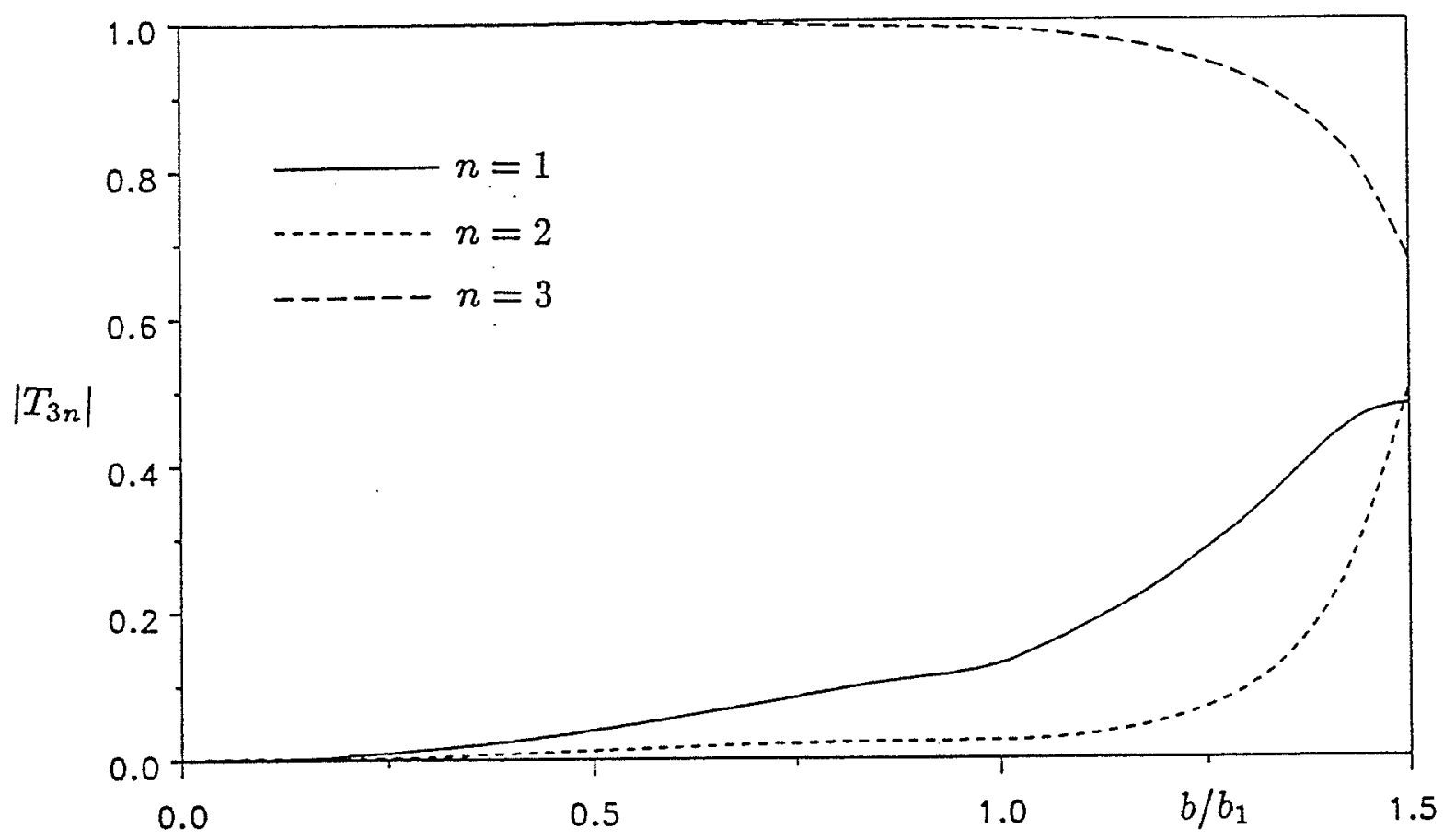

(a)

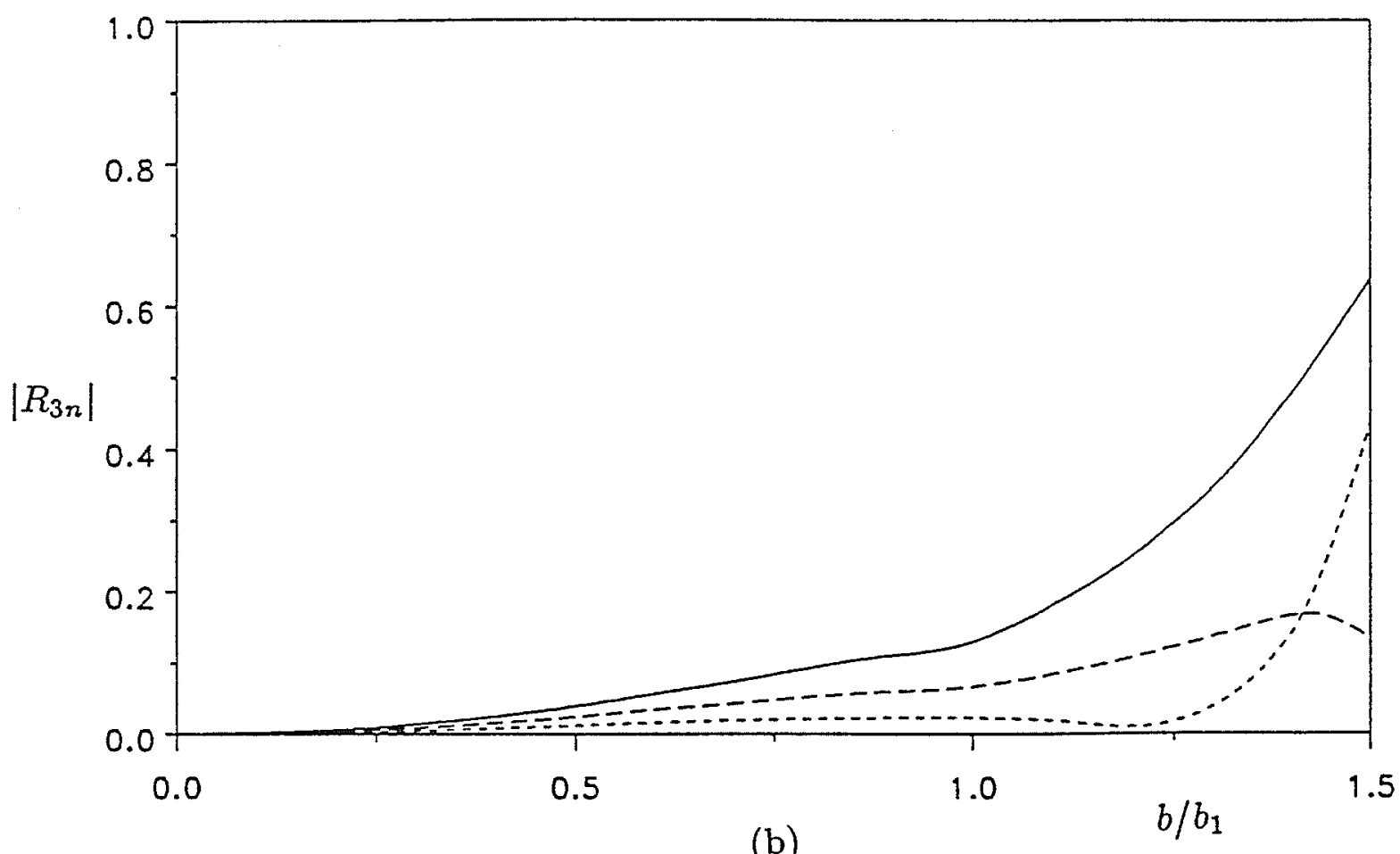

(b)

Figure 4.16: Variation of $\left|T_{3 n}\right|$ and $\left|R_{3 n}\right|$ with normalized crack length at $\Omega=4.0$ for example 4, due to third antisymmetric incident mode (plane strain case):
(a) $\left|T_{3 n}\right|$ vs $b / b_{1}$
(b) $\left|R_{3 n}\right|$ vs $b / b_{1}$. 


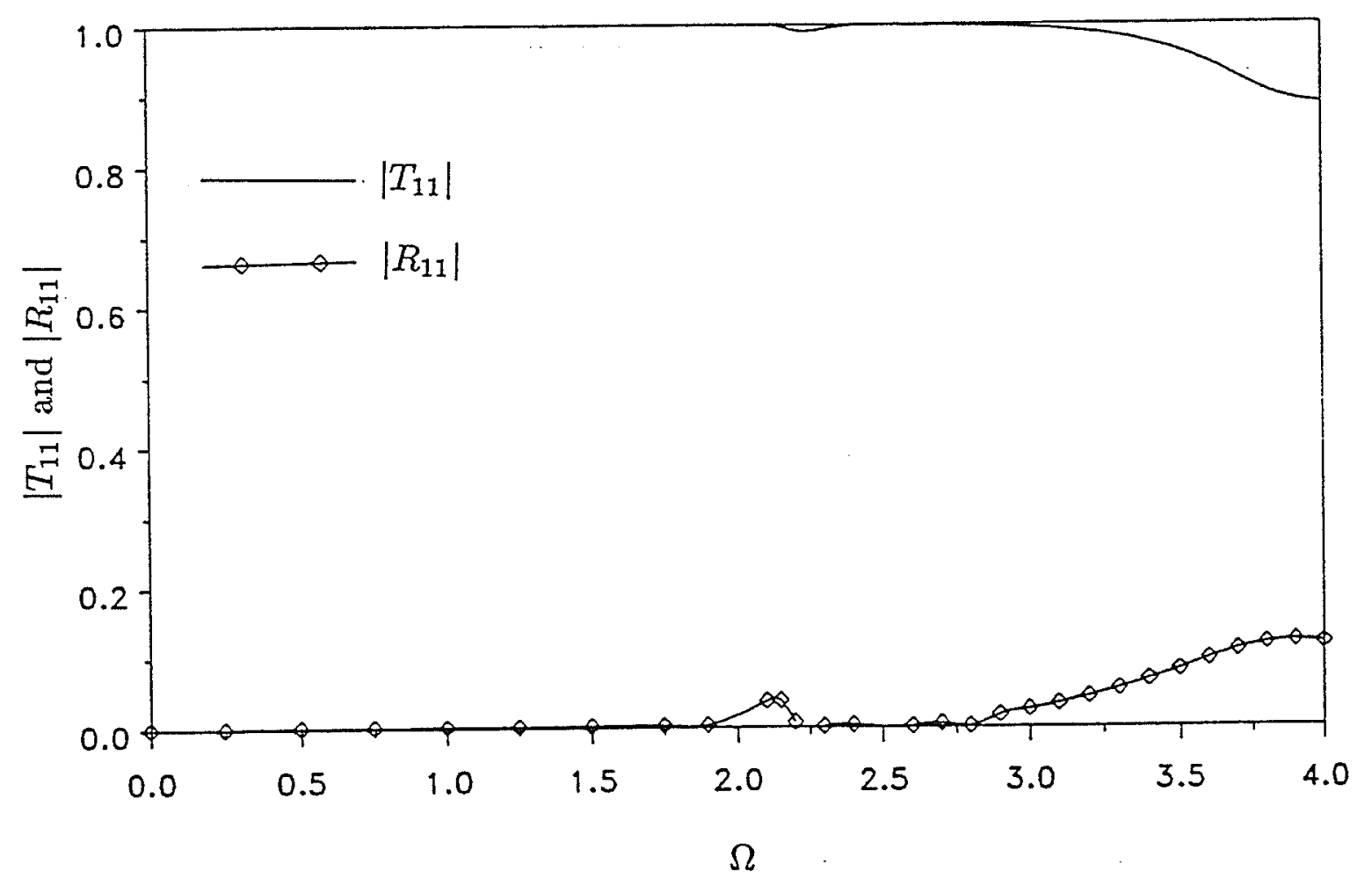

Figure 4.17 : Variation of $\left|T_{11}\right|$ and $\left|R_{11}\right|$ with $\Omega$ for example 4 , due to first symmetric incident mode, when $b / b_{1}=1.0$ (plane strain case). 


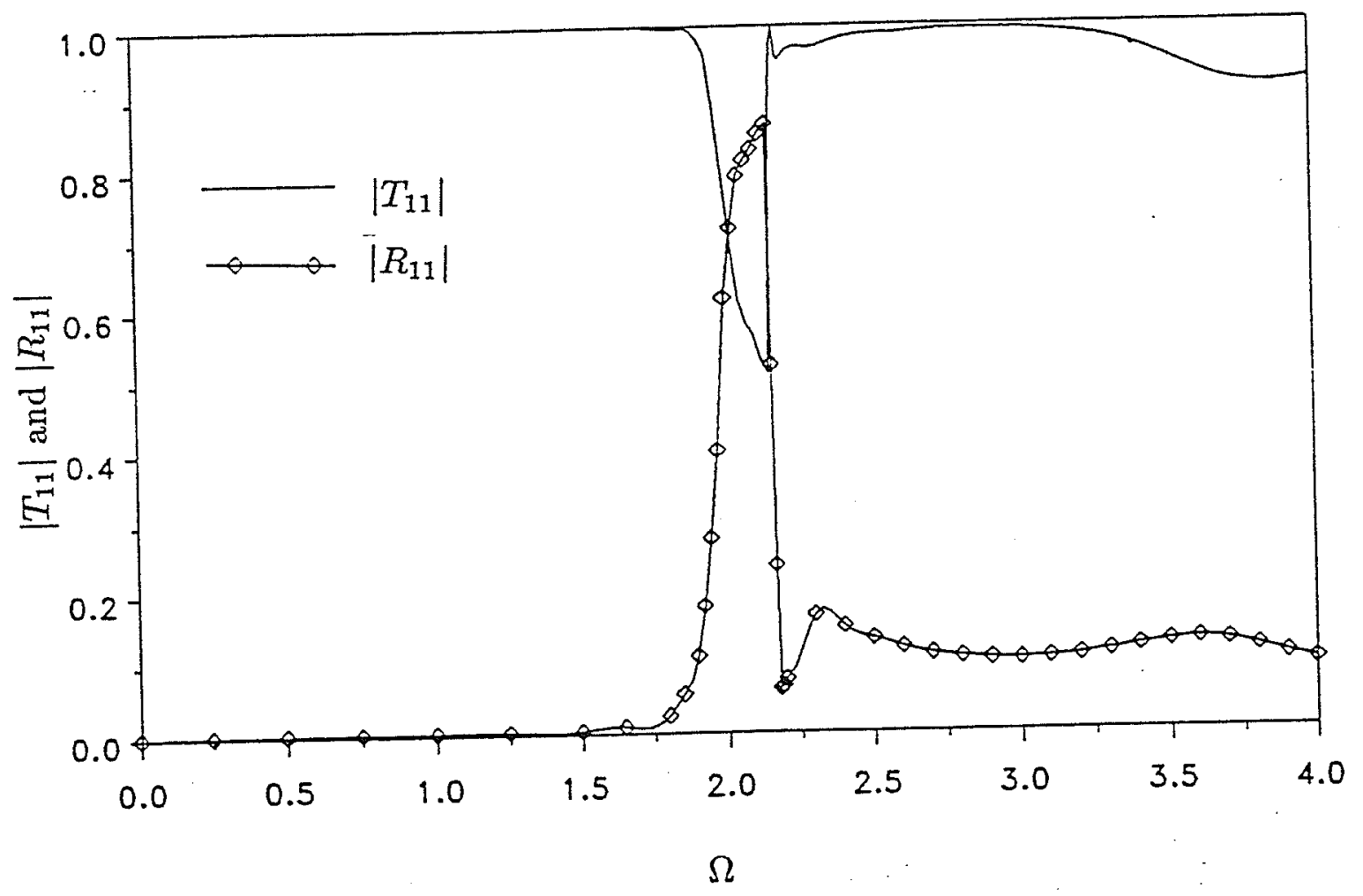

Figure 4.18: Variation of $\left|T_{11}\right|$ and $\left|R_{11}\right|$ with $\Omega$ for example 4 , due to first symmetric incident mode, when $b / b_{1}=1.5$ (plane strain case). 


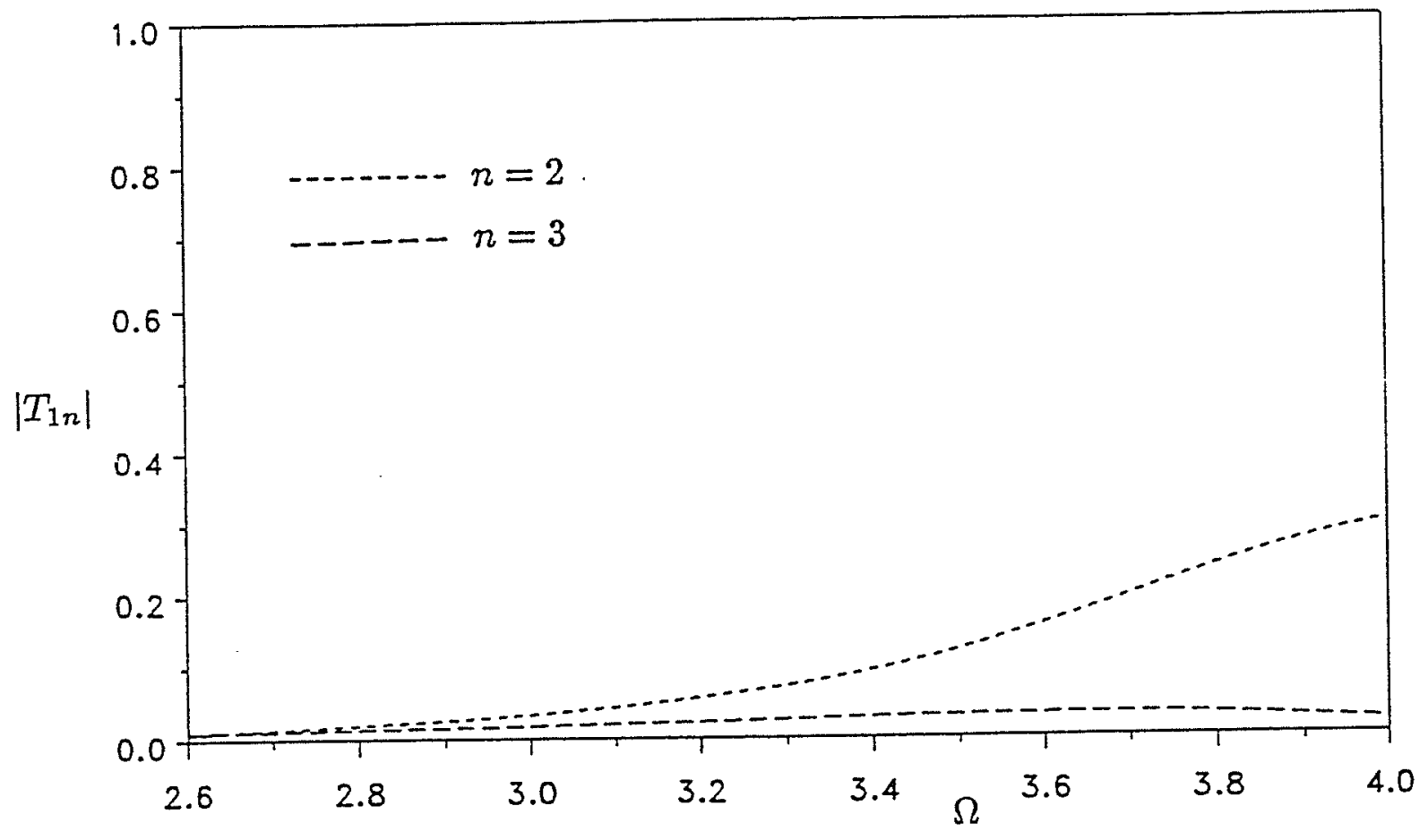

(a)

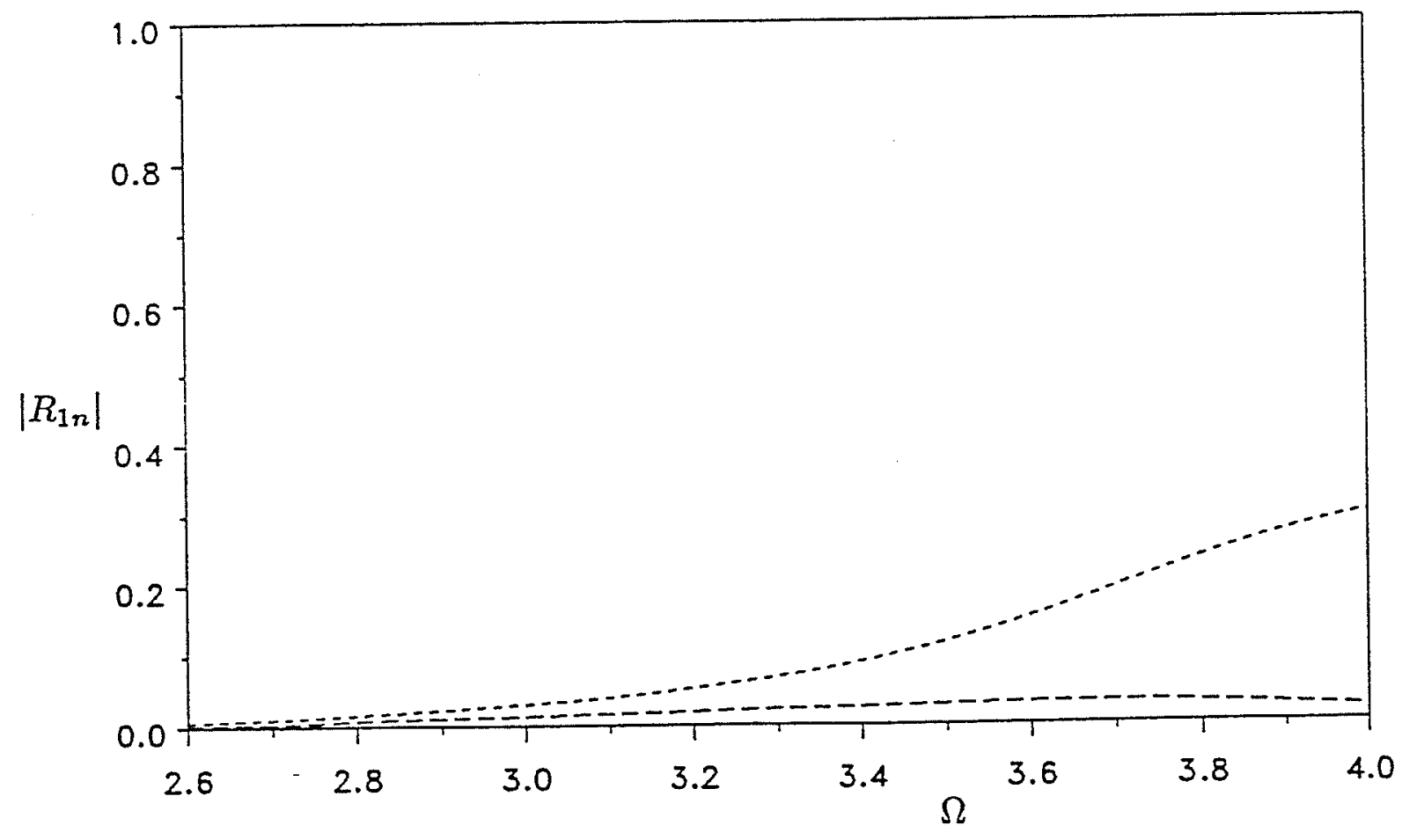

(b)

Figure 4.19 : Variation of $\left|T_{1 n}\right|$ and $\left|R_{1 n}\right|$ with $\Omega$ for example 4, due to first symmetric incident mode, when $b / b_{1}=1.0$ (plane strain case):
(a) $\left|T_{1 n}\right|$ vs $\Omega$,
(b) $\left|R_{1 n}\right|$ vs $\Omega$. 


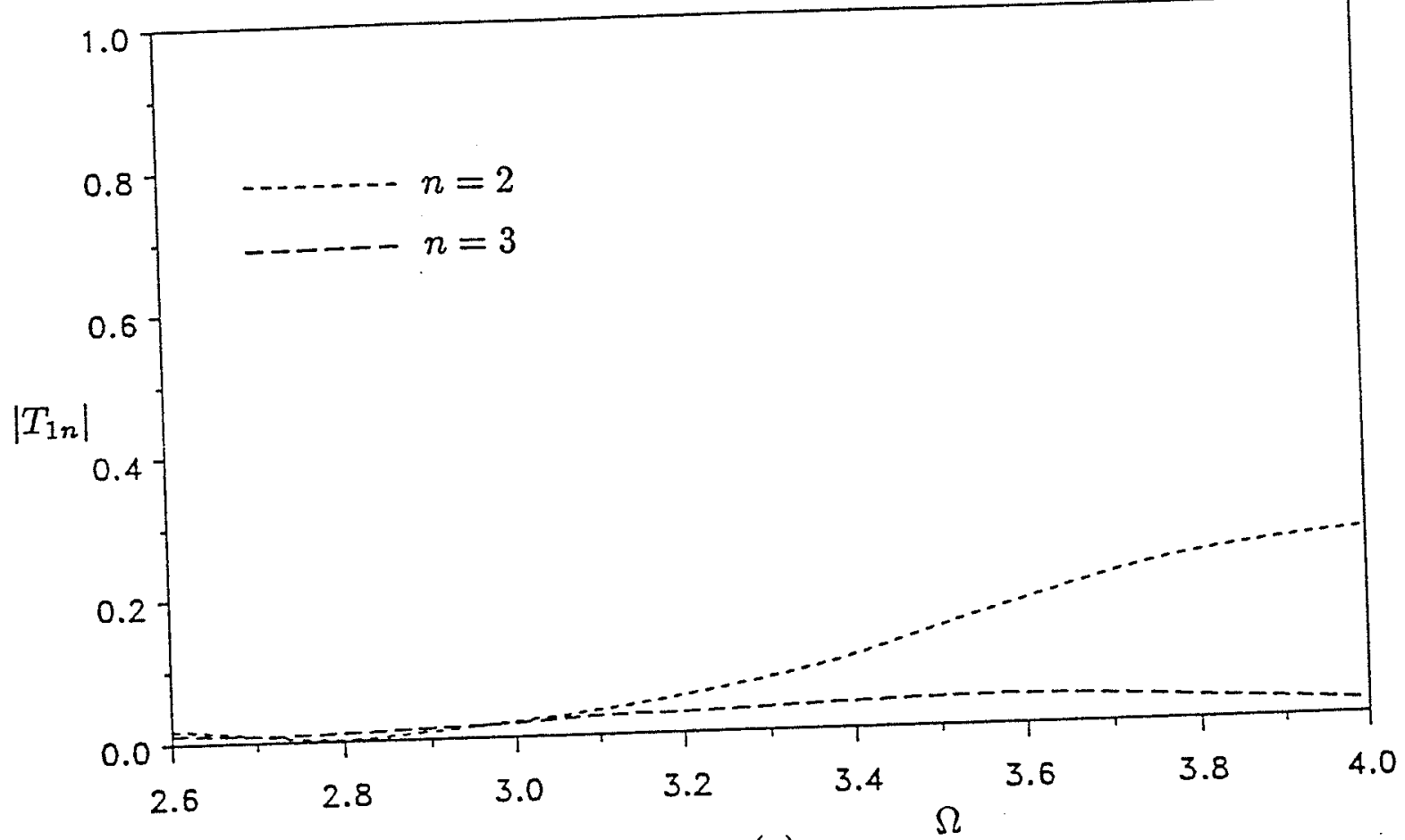

(a)

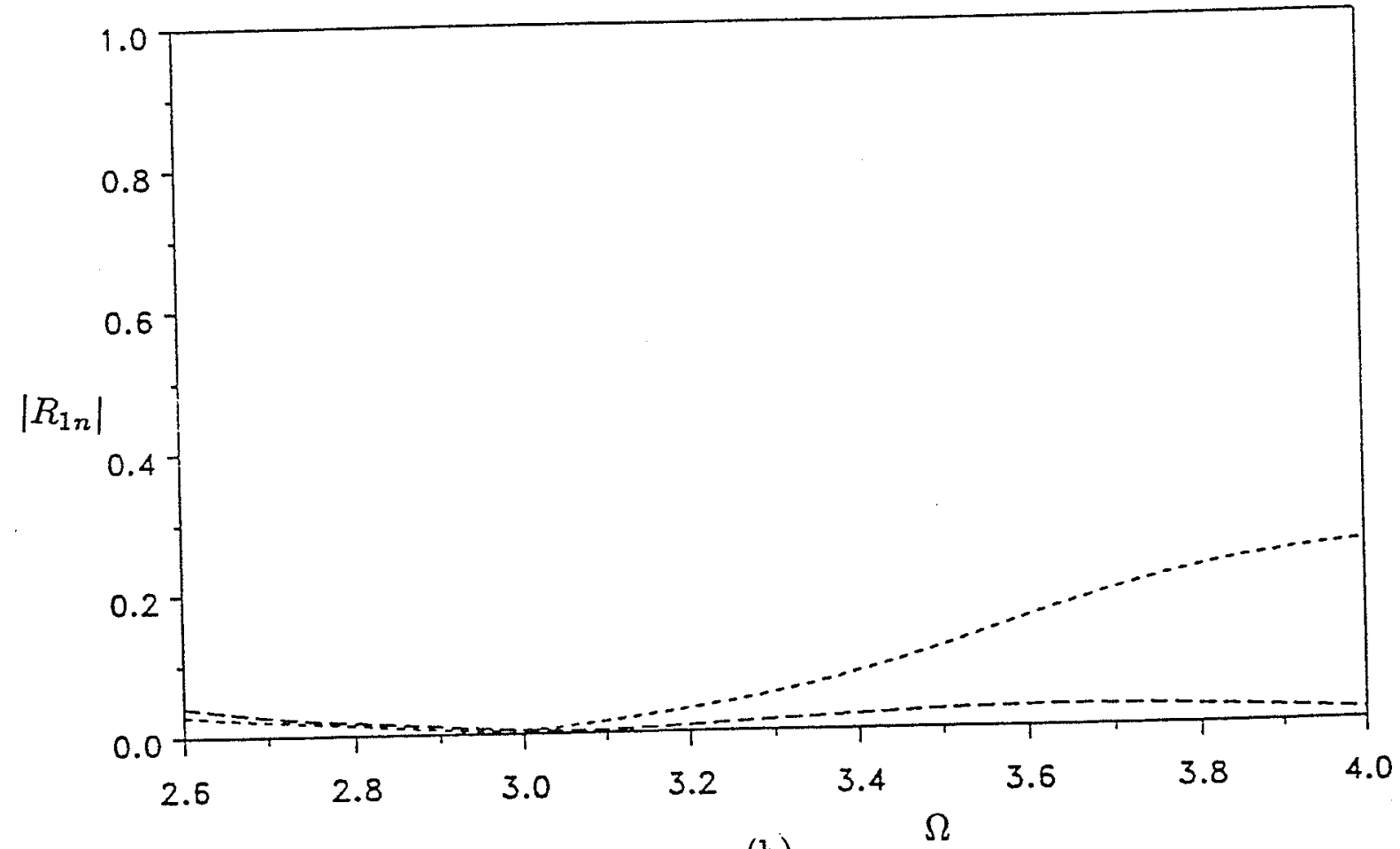

(b)

Figure 4.20: Variation of $\left|T_{1 n}\right|$ and $\left|R_{1 n}\right|$ with $\Omega$ for example 4, due to first symmetric incident mode, when $b / b_{1}=1.5$ (plane strain case):
(a) $\left|T_{1 n}\right|$ vs $\Omega$,
(b) $\left|R_{1 n}\right|$ vs $\Omega$. 


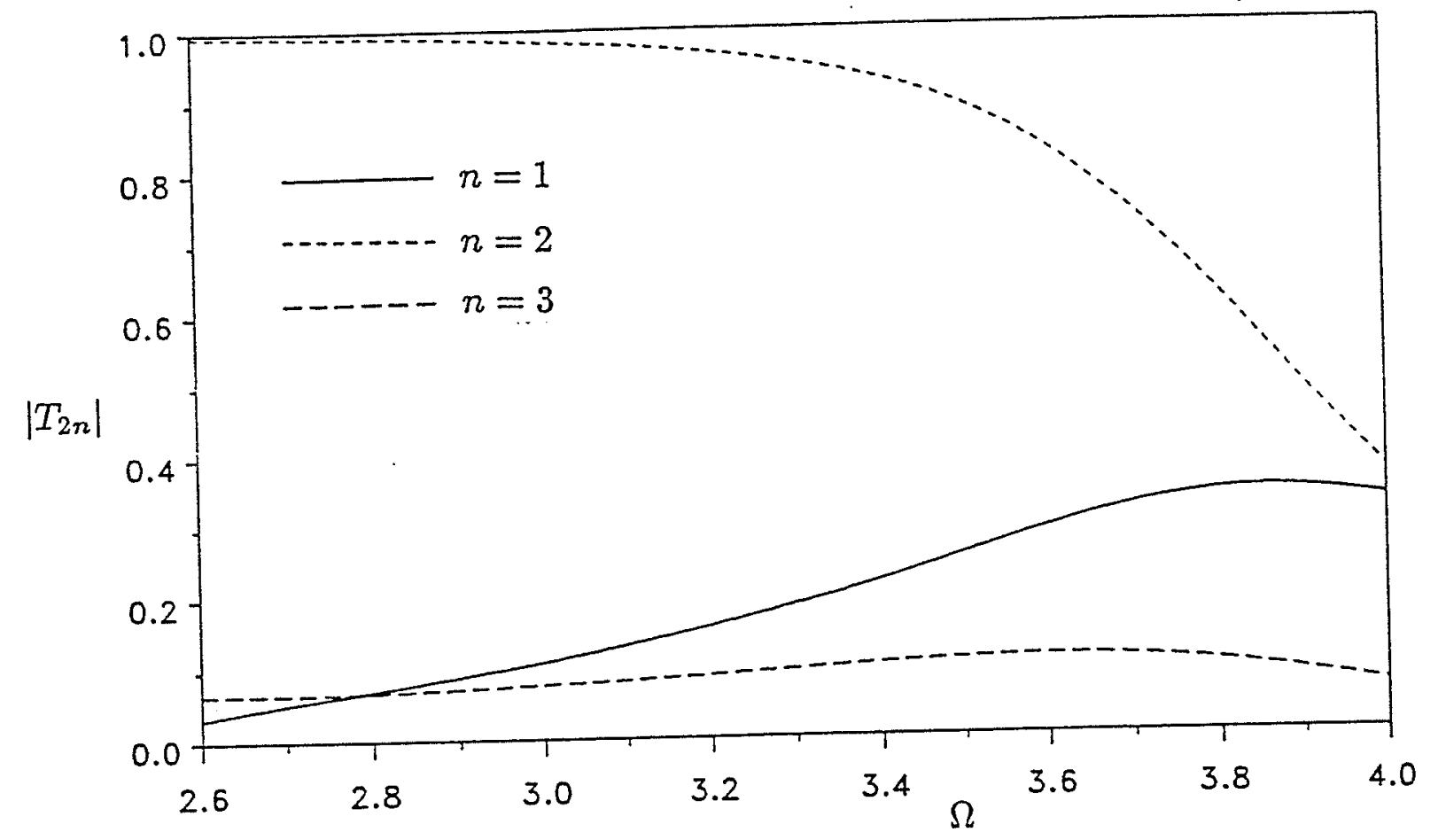

(a)

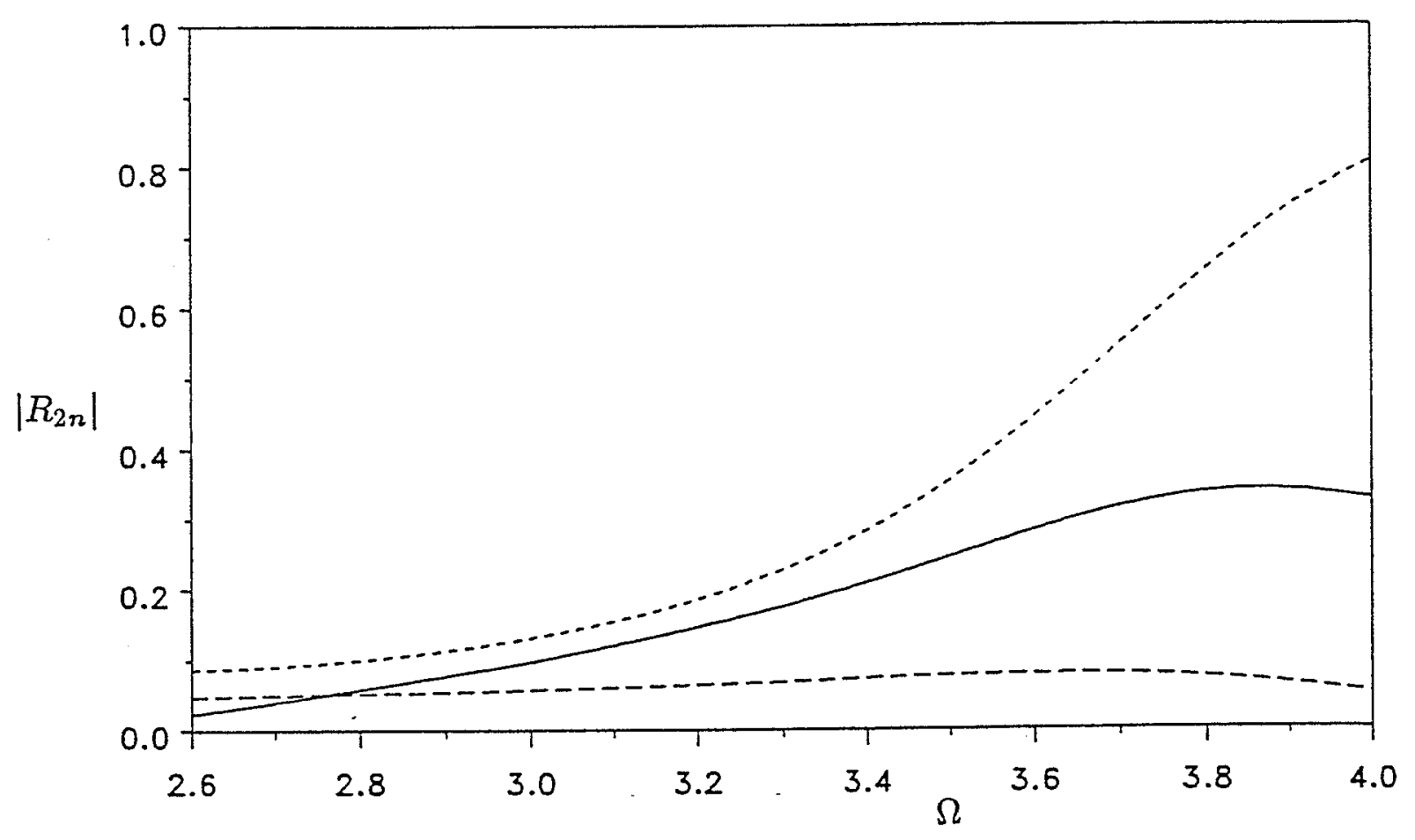

(b)

Figure 4.21: Variation of $\left|T_{2 n}\right|$ and $\left|R_{2 n}\right|$ with $\Omega$ for example 4, due to second symmetric incident mode, when $b / b_{1}=1.0$ (plane strain case):
(a) $\left|T_{2 n}\right|$ vs $\Omega$,
(b) $\left|R_{2 n}\right|$ vs $\Omega$. 

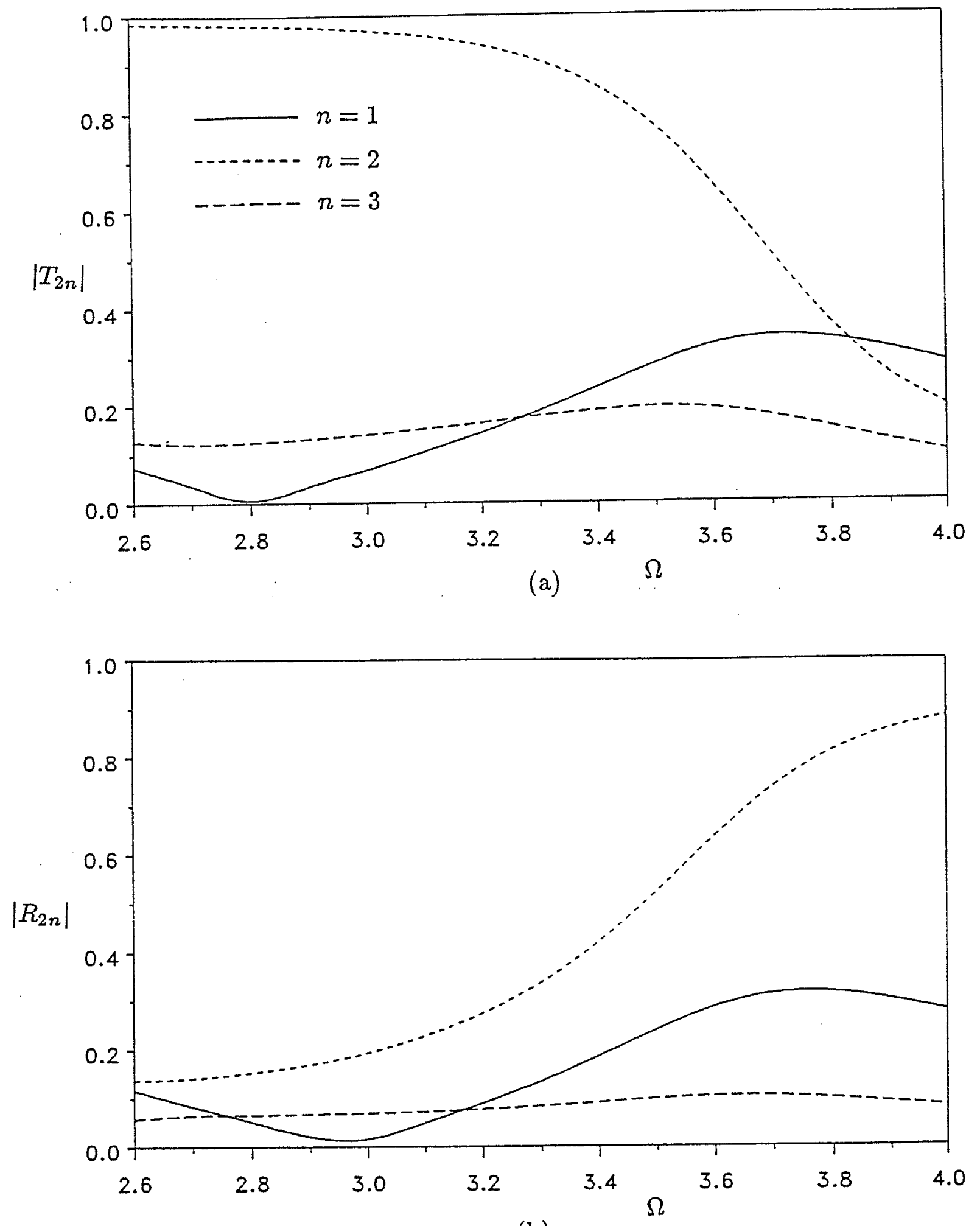

(b)

Figure 4.22 : Variation of $\left|T_{2 n}\right|$ and $\left|R_{2 n}\right|$ with $\Omega$ for example 4, due to second symmetric incident mode, when $b / b_{1}=1.5$ (plane strain case):
(a) $\left|T_{2 n}\right|$ vs $\Omega$,
(b) $\left|R_{2 n}\right|$ vs $\Omega$. 


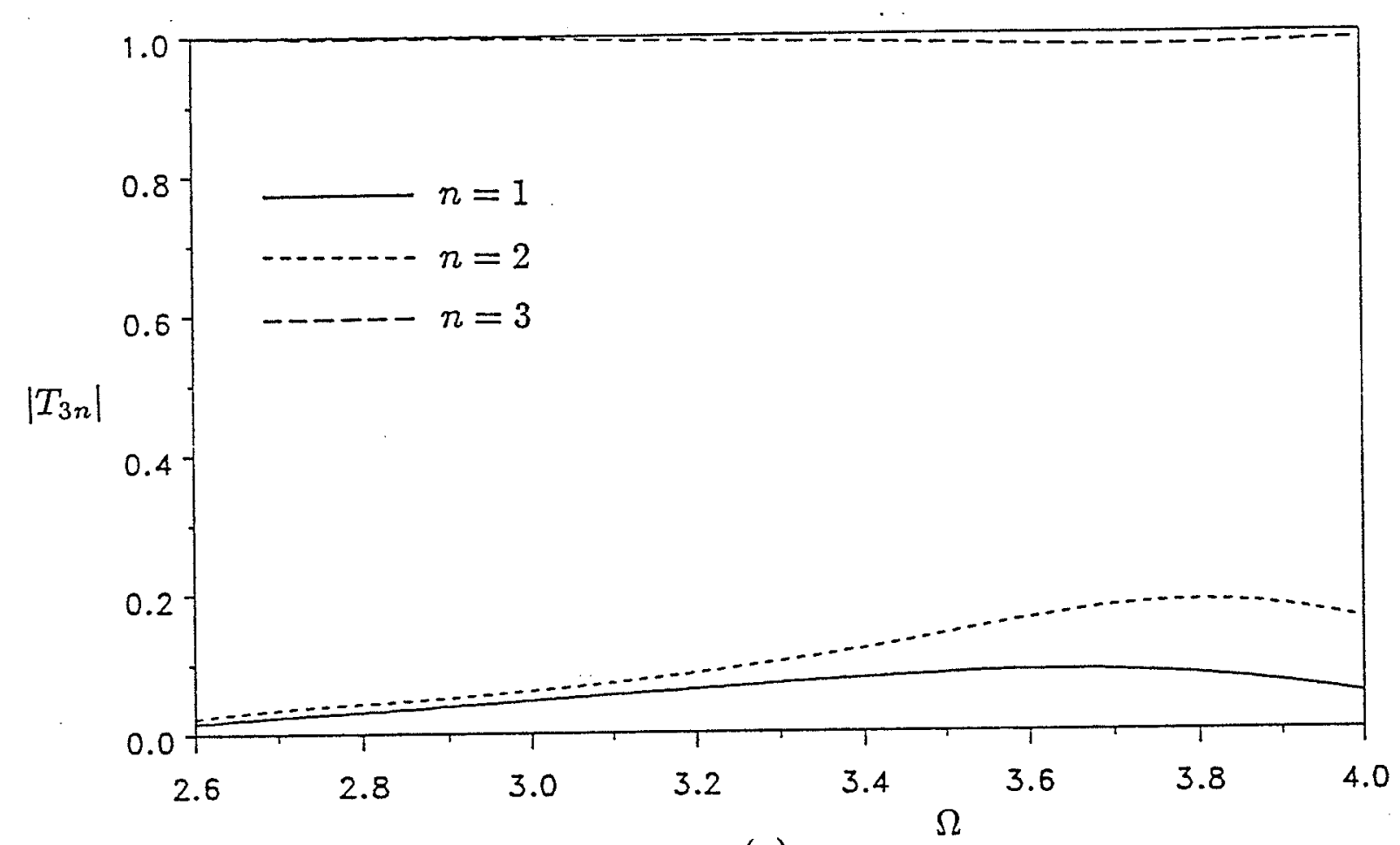

(a)

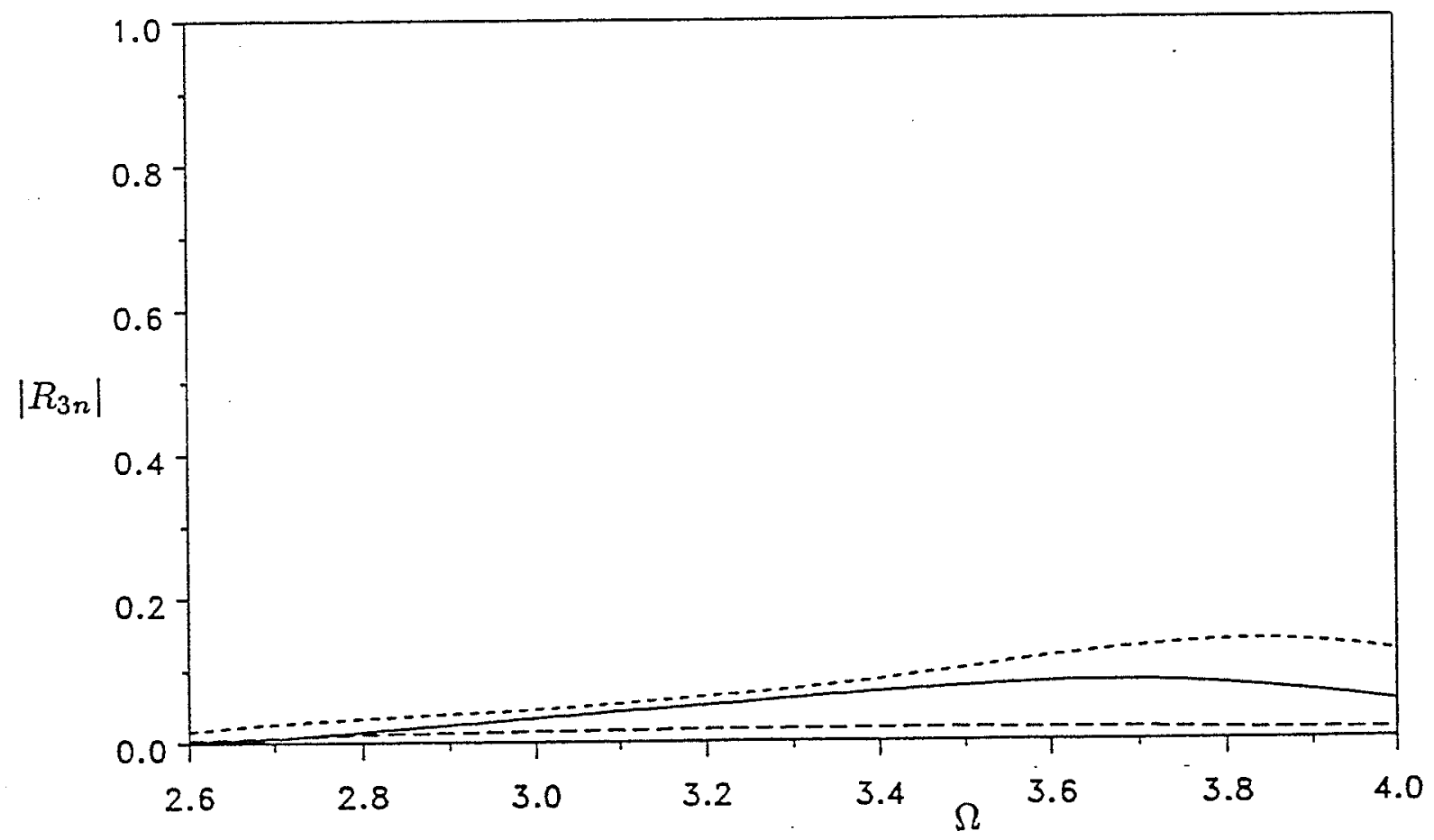

(b)

Figure 4.23 : Variation of $\left|T_{3 n}\right|$ and $\left|R_{3 n}\right|$ with $\Omega$ for example 4, due to third symmetric incident mode, when $b / b_{1}=1.0$ (plane strain case):

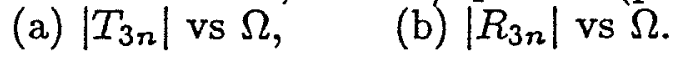




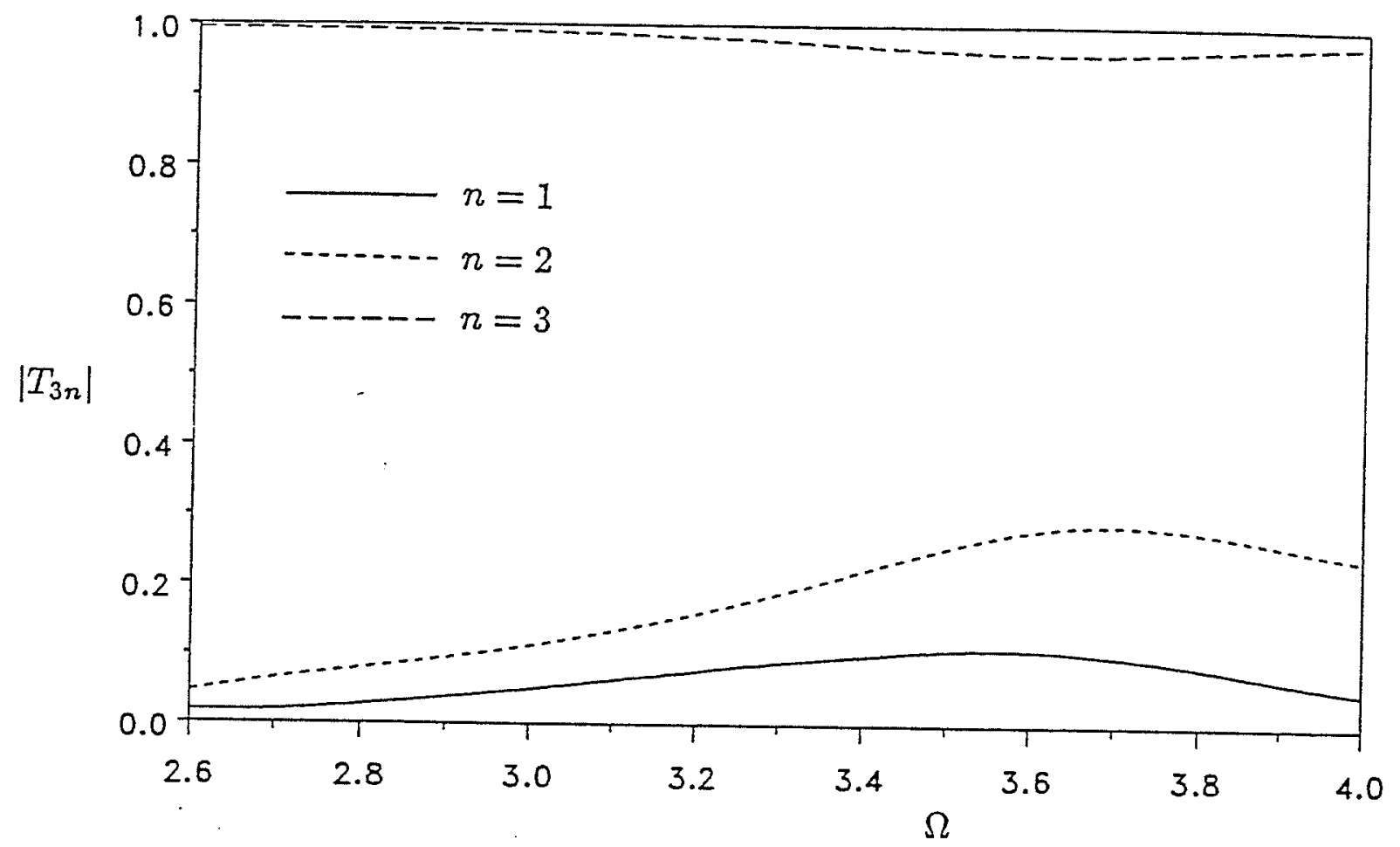

(a)

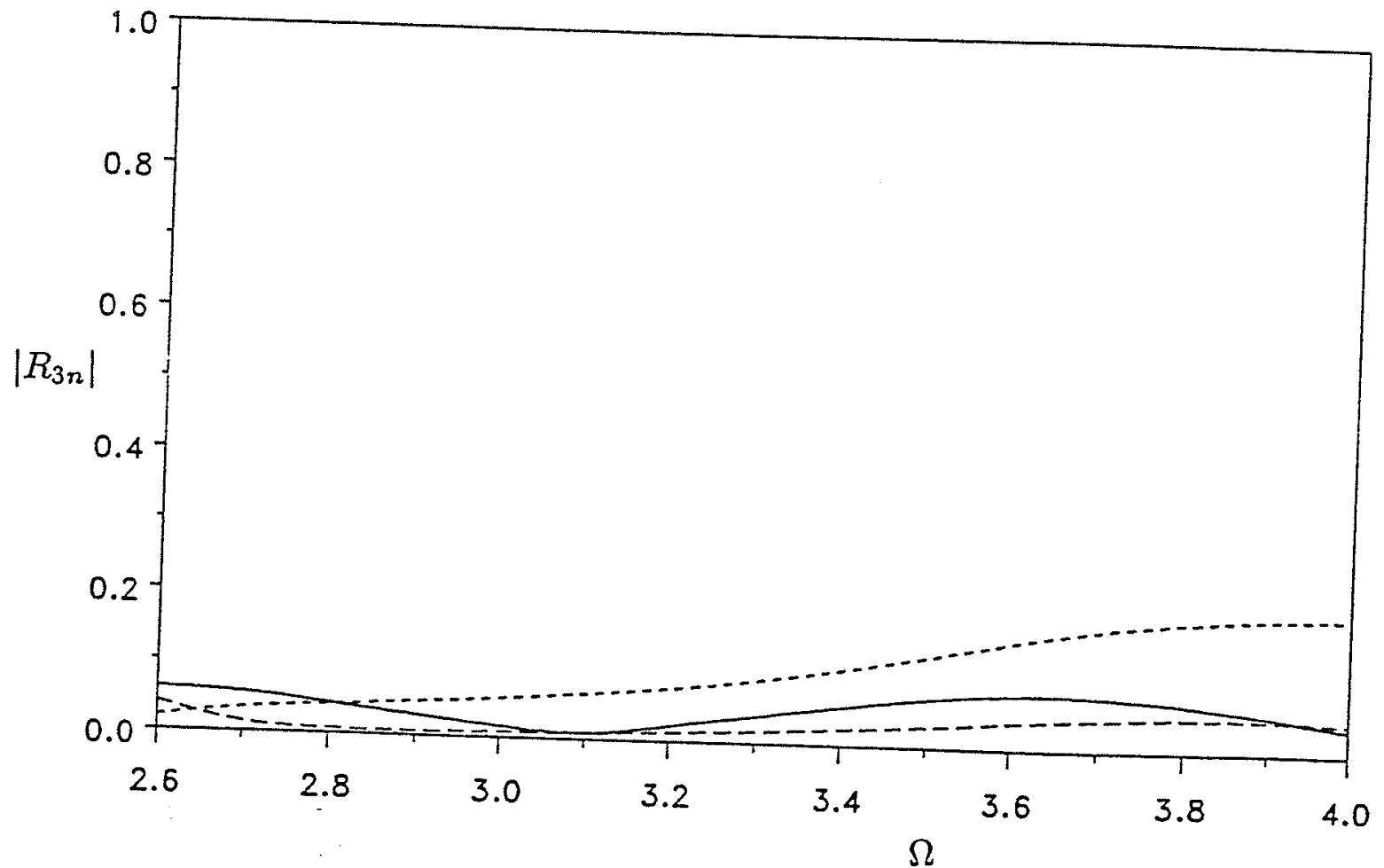

(b)

Figure 4.24: Variation of $\left|T_{3 n}\right|$ and $\left|R_{3 n}\right|$ with $\Omega$ for example 4 , due to third symmetric incident mode, when $b / b_{1}=1.5$ (plane strain case):
(a) $\left|T_{3 n}\right|$ vs $\Omega$,
(b) $\left|R_{3 n}\right|$ vs $\Omega$. 
Table 4.1

Numerical results for the scattering problem in example 2 at $\Omega=2.0$

(general case):

(a) $\theta=0^{\circ}, \phi^{\text {in }}($ when $p=1)=45^{\circ}, \zeta_{0}=1.4404$.

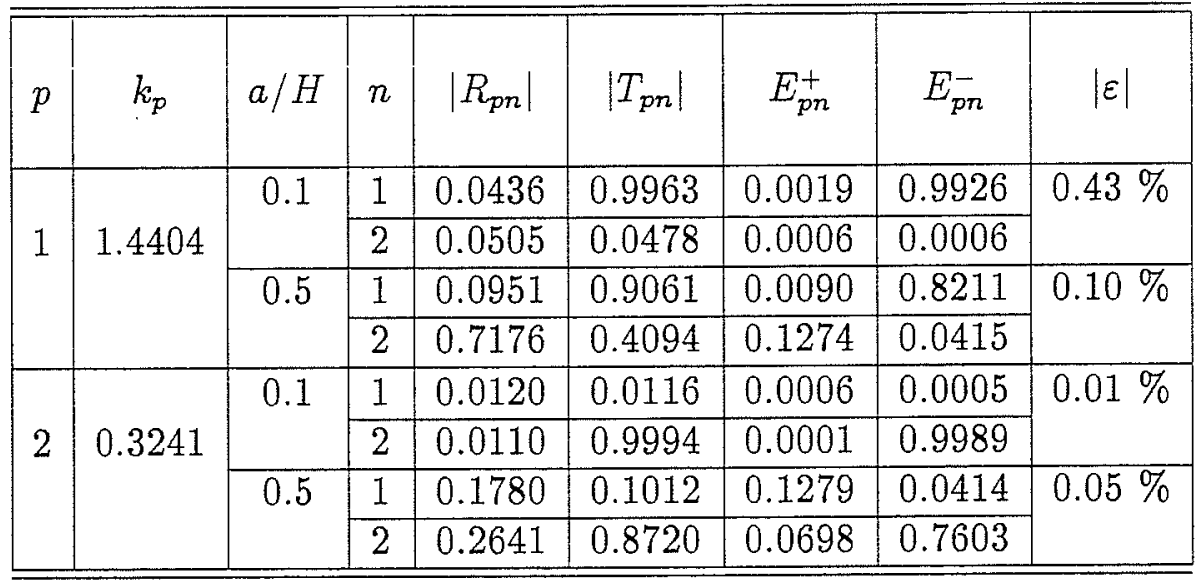

(b) $\theta=22.5^{\circ}, \phi^{\text {in }}($ when $p=1)=22.5^{\circ}, \zeta_{0}=0.7796$.

\begin{tabular}{|c|c|c|c|c|c|c|c|c|}
\hline$p$ & $k_{p}$ & $a / H$ & $n$ & $\left|R_{p n}\right|$ & $\left|T_{p n}\right|$ & $E_{p n}^{+}$ & $E_{p n}^{-}$ & $\mid \varepsilon$ \\
\hline \multirow{4}{*}{1} & \multirow{4}{*}{1.8820} & \multirow[t]{2}{*}{0.1} & 1 & 0.0092 & 0.9987 & 0.0000 & 0.9975 & \multirow[t]{2}{*}{$0.21 \%$} \\
\hline & & & 2 & 0.0619 & 0.0325 & 0.0002 & 0.0002 & \\
\hline & & \multirow[t]{2}{*}{0.5} & 1 & 0.1051 & 0.9129 & 0.0016 & 0.8334 & \multirow[t]{2}{*}{$0.21 \%$} \\
\hline & & & 2 & 1.3568 & 0.6322 & 0.0811 & 0.0818 & \\
\hline \multirow{4}{*}{2} & \multirow{4}{*}{0.8129} & \multirow[t]{2}{*}{0.1} & $\overline{1}$ & 0.0136 & 0.0065 & 0.0001 & 0.0002 & \multirow[t]{2}{*}{$0.01 \%$} \\
\hline & & & 2 & 0.0087 & 0.9999 & 0.0000 & 0.9997 & \\
\hline & & \multirow[t]{2}{*}{0.5} & 1 & 0.0240 & 0.1294 & 0.0004 & 0.0819 & \multirow[t]{2}{*}{$0.09 \%$} \\
\hline & & & 2 & 0.5902 & 0.9175 & 0.0751 & 0.8419 & \\
\hline
\end{tabular}

Notes : 1) $p$ and $n$ denote the incident and scattered wave node numbers, respectively.

2) Symmetric incident wave modes have been considered. 
Table 4.2

Numerical results for the scattering problem in example 5 at $\Omega=4.0$ and

$$
a / H=0.5 \text { (general case): }
$$

(a) $\theta\left(\right.$ for $0^{\circ}$ lamina $)=0^{\circ}, \phi^{\text {in }}($ when $p=1)=45^{\circ}, \zeta_{0}=3.3624$.

\begin{tabular}{|c|c|c|c|c|c|c|c|}
\hline \hline$p$ & $k_{p}$ & $n$ & $\left|R_{p n}\right|$ & $\left|T_{p n}\right|$ & $E_{p n}^{+}$ & $E_{p n}^{-}$ & $|\varepsilon|$ \\
\hline \multirow{2}{*}{1} & 3.3624 & 1 & 0.8401 & 0.4240 & 0.7058 & 0.1797 & $0.04 \%$ \\
\cline { 3 - 7 } & & 2 & 0.1432 & 0.5706 & 0.0068 & 0.1081 & \\
\hline \multirow{2}{*}{2} & \multirow{2}{*}{0.6622} & 1 & 0.0466 & 0.1893 & 0.0066 & 0.1080 & $0.25 \%$ \\
\cline { 3 - 6 } & & 2 & 0.7370 & 0.5828 & 0.5432 & 0.3397 & \\
\hline
\end{tabular}

(b) $\theta\left(\right.$ for $0^{\circ}$ lamina $)=22.5^{\circ}, \phi^{\text {in }}($ when $p=1)=22.5^{\circ}, \zeta_{0}=1.8197$.

\begin{tabular}{|c|c|c|c|c|c|c|c|}
\hline$p$ & $k_{p}$ & $n$ & $\left|R_{p n}\right|$ & $\left|T_{p n}\right|$ & $E_{p n}^{+}$ & $E_{p n}^{-}$ & $|\varepsilon|$ \\
\hline & \multirow{3}{*}{4.3931} & 1 & 0.3686 & 0.3171 & 0.6417 & 0.1006 & \multirow{3}{*}{$0.02 \%$} \\
\hline & & 2 & 0.4614 & 0.0768 & 0.1640 & 0.0394 & \\
\hline & & 3 & 0.1963 & 0.1072 & 0.0472 & 0.0073 & \\
\hline \multirow{3}{*}{2} & \multirow{3}{*}{1.9389} & 1 & 0.1308 & 0.5111 & 0.0121 & 0.0391 & \multirow{3}{*}{$0.08 \%$} \\
\hline & & 2 & 0.9173 & 0.8243 & 0.0970 & 0.6795 & \\
\hline & & 3 & 0.0297 & 1.3467 & 0.0002 & 0.1730 & \\
\hline \multirow{3}{*}{3} & \multirow{3}{*}{1.4683} & 1 & 0.1726 & 0.0687 & 0.2209 & 0.0074 & \multirow{3}{*}{$0.47 \%$} \\
\hline & & 2 & 0.6732 & 0.1283 & 0.5479 & 0.1725 & \\
\hline & & 3 & 0.0874 & 0.2032 & 0.0147 & 0.0413 & \\
\hline
\end{tabular}

Notes : 1) $p$ and $n$ denote the incident and scattered wave node numbers, respectively.

2) Symmetric incident wave modes have been considered. 


\section{Conclusions, and recommendations for Future Work}

\subsection{Conclusions}

The stiffness method, developed in Chapter 2, can be used to obtain accurate predictions of theoretical dispersion characteristics that are required for material characterisation of laminated composite plates with arbitrary number of layers. It is found that measurable changes in phase velocity are caused by interface bond layers. These changes are quite appreciable at high frequencies and specially in higher modes within certain frequency bands. The present investigation suggests not only that ultrasonic waves may be used to characterise interface bond layer properties but also that judicious choice of frequency and modes may be made to obtain optimum results in ultrasonic nondestructive evaluation of these properties. The study shows that anisotropy, caused by the orientation of fibers with respect to the direction of wave propagation, has a strong influence on dispersion characteristics. It is shown that the number of layers in a cross-ply laminated plate has a strong influence on the dispersion characteristics of guided waves when the plate is composed of only a few

layers. However, as the number of layers increases, the layering effects diminish, and when there is a sufficiently large number of layers, the effective mechanical properties of the cross-ply plate can be characterised as homogeneous anisotropic. This finding is very important from the point of view of designing thick laminated composite plates, since the designer can use a homogeneous plate model rather than a complicated layered plate model.

The semi-analytical method, developed in Chapter 3, can be used to study 
guided plane strain wave reflection at the free edge of a laminated composite plate with arbitrary number of layers. Although the case of reflection of waves that propagate along a principle direction has been studied, the method presented in this chapter can be easily applied to off-axis propagation. It is found that the variational principle method gives very good results. Since the least-squares method gives anomalous results form the point of view of energy balance, it is concluded that the least-squares method should be used with caution for free end reflection problem of layered anisotropic plates.

The hybrid method, presented in Chapter 4 to investigate scattering by cracks and delaminations in the laminated composite plates, has several advantages: each lamina of the plate can have arbitrary mechanical properties and thickness; because the scattered field is expressed in wave function expansion, an arbitrary number of layers can be accommodated without appreciable increase in computational time; the interior region containing the flaw can have quite arbitrary material properties; the flaw can be arbitrary in geometry and in orientation; and the multiple scattering by a cluster of neighbouring flaws can be studied without much difficulty. Although the wave function expansion has been presented for laminated plates having orthotropic or transversely isotropic lamina properties, the hybrid method can be easily adapted to suit laminated plates having general anisotropic lamina properies. It is found that the reflection and transmission coefficients are very sensitive to the extent and the orientation of the flaw, the incident mode number, and the frequency. The present study enables the experimentalist to make judicious choice of the frequency and the incident mode to obtain optimum results in ultrasonic nondestructive evaluation of flaws. Futhermore, the hybrid formulation based finite element code, developed in the present study, enables one to perform a detailed parametric study of the scattering problem, and the results of this para- 
metric study may be used to interpret ultrasonic test measurements thus leading to the evaluation of the parameters of the flaw nondestructively. The hybrid method is ideally suited for low frequencies. At high frequencies, very fine finite element meshes have to be used, thus increasing the number of degrees of freedoms involved in the analysis. It is noted here that the scattering problem considered in the thesis is a highy idealised one. Therefore, for the technique to be useful for practical applications, a considerable amount of experimental work and additional theoretical study is required.

\subsection{Recommendations for Future Work}

It is recommended that the theoretical findings of the present study be experimentally validated by carrying out ultrasonic testing of laminated composite plate specimens. This goal may be acheived by testing specimens with accurately known, material properties and flaw parameters. In the hybrid formulation presented in Chapter 4, only time harmonic waves have been considered. In addition, it has been assumed that the source or the transducer which excites guided waves is located at a distance far away from the flaw, and the flaw is of infinite length in $y$ direction. The following recommendations are made for future work for the hybrid method to be useful for ultrasonic nondestructive evaluation of flaws in laminated composite plates:

1. Incorporate transient waves in the hybrid method, by employing the Fourier Transform Technique. This will permit to investigate the possibility of using the time pulses in ultrasonic nondestructive evaluation.

2. Incorporate a guided wave generating transducer located at an arbitrary distance from the flaw. This may be possible, by enclosing the flaw and the 
region loaded by the transducer, by two interior regions: one enclosing the loaded region; and the other enclosing the flaw. In between the two interior regions, there is an exterior region which can be modeled by the wave function expansion procedure.

3. Extend the hybrid method to investigate scattering by finite flaws (i.e. 3dimensional flaws).

The hybrid technique presented in this thesis is a direct method having potential applications in determinig flaw parameters from ultrasonic measurements. However, if the number of unknown flaw parameters involved is quite large, application of this direct method to quantify unknown parameters may become very tedious. To circumvent this difficulty it may be necessary to study the inverse scattering problem. The recent work by Nishimura and Kobayashi (1991) on SH problems is a starting point for future research in this direction. 


\section{References}

Abduljabbar, Z., Datta, S.K., and Shah A.H. (1983). "Diffraction of horizontally polarized shear waves by normal edge cracks in a plate." J. Appl. Phys., 54(2), 461-472.

Achenbach, J.D. (1973). Wave Propagation in Elastic Solids, North-Holland Publishing Company, Amsterdam.

Achenbach, J.D. and Kitahara, M. (1986). "Reflection and transmission of an obliquely incident wave by an array of spherical cavities." J. Acoust. Soc. Am., 80, 1209-1214.

Achenbach, J.D. and Li, Z.L. (1986). "Propagation of horizontally polarized tranverse waves in a solid with a periodic distribution of cracks." Wave Motion, 8, $371-379$.

Al-Nassar (1990). "Scattering of lamb waves in an elastic plate for ultrasonic nondestructive evaluation of weldments and cracks." Ph.D. Thesis, Dept. of Mech. Eng., University of Colorado, Boulder, Colorado, U.S.A.

Angle, Y.C. and Achenbach, J.D. (1987). "Harmonic waves in an elastic solid containing a doubly periodic array of cracks." Wave Motion, 9, 377-385.

Auld, B.A. (1973). Acoustic Fields and Waves in solids, 2, Wiley-Interscience, New York.

Barsoum, R.S. (1976). "On the use of isoparametric finite elements in linear fracture mechanics." Int. J. Numer. Meth. Eng., 10, 25-37.

Barsoum, R.S. (1988). "Application of the finite element iterative method to the eigenvalue problem of a crack between dissimilar media." Int. J. Numer. Meth. Eng., 26, 541-554. 
Bathe, K.J. (1982). Finite Element Procedures in Engineering Analysis, PrenticeHall Inc., New Jersey.

Baylis, E.R. and Green, W.A. (1986a). "Flexural waves in fiber-reinforced laminated plates." J. Sound and Vibration, 110(1), 1-26.

Baylis, E.R. and Green, W.A. (1986b). "Flexural waves in fiber-reinforced laminated plates, Part II." J. Sound and Vibration, 111(2), 181-190.

Baylis, E.R. (1988). "Wave propagation in asymmetric fiber-reinforced laminated plate, Part II." Acta Mechanica, 71, 167-183.

Berryman, J.G. (1980a). "Long-wavelength propagation in composite elastic MediaI Spherical inclusions." J. Acoust. Soc. Am., 68(6), 1809-1819.

Berryman, J.G. (1980b). "Long-wavelength propagation in composite elastic MediaII Ellipsoidal inclusions." J. Acoust. Soc. Am., 68(6), 1820-1831.

Bratton, R.L., Datta, S.K. and Shah, A.H. (1990). "Propagation and scattering of Lamb waves in a fibre-rienforced composite plate." Report 90-1, Center for space construction, University of Colorado, Boulder.

Chimenti, D. and Nayfeh, A.H. (1990). "Ultrasonic reflection and guided wave propagation in biaxially laminated composite plates." J. Acoust. Soc. Am., $87(4), 1409-1415$.

Conte, S.D., and Boor, C.D. (1972). Elementary Numerical Analysis, 2nd Edn. Mc Graw-Hill, New York.

Datta, S.K. (1977). "A self-consistent approach to multiple scattering of elastic waves." J. Appl. Mech., 44, 657-662.

Datta, S.K., Achenbach, J.D., and Rajapakse, Y.S., eds. (1990a). Elastic waves and ultrasonic nondestructive evaluation, Elsevier Science Publishers, Amsterdam, 
North Holland.

Datta, S.K., and Ledbetter, H.M. (1983). "Elastic constants of fiber-reinforced boron-aluminium: Observations and Theory." Int. J. Solids Struct., 19(10), 885894.

Datta, S.K., Ledbetter, H.M. and Kriz, R. (1984). "Calculated elastic constants of composites containing anisotropic fibers." Int. J. Solids Sruct., 20(5), 429-438.

Datta, S.K. and Ledbetter, H.M. (1987). "Ultrasonic characterization of material properties of composite materials." Proceedings of the tenth U.S. National Congress of Applied Mechanics, ASME, (edited by Lamb, J.P.) New York, 377387.

Datta, S.K., Shah, A.H., Bratton, R.L., and Chakraborthy, T. (1988). "Wave propagation in laminated composite plates." J. Acoust. Soc. Am., 83(6), 20202026 .

Datta, S.K., Shah, A.H., and Karunasena, W. (1990b). "Wave propagation in composite media and material characterization." Elastic waves and nondestructive evaluation (edited by Datta, S.K, Achenbach, J.D., and Rajapakse, Y.S.), Amsterdam, North-Holland.

Dong, S.B., and Huang, K.H. (1985). "Edge vibrations in laminated composite plates." J. Appl. Mech., 52, 433-438.

Dong, S.B. and Nelson, R.B. (1972). "On natural vibrations and waves in laminated orthotropic plates." J. Appl. Mech., 39, 739-745.

Dong, S.B. and Pauley, K.E. (1978), "Plane waves in anisotropic plates." J. Eng. Mech., 104, 801-817.

Fenner, D.N. (1976). "Stress singularities in composite materials with an arbitrarily oriented crack meeting an interface." Int. J. Fract., 12(5), 705-721. 
Gregory, R.D. and Gladwell, I. (1983). "The reflection of a symmetric RayleighLamb wave at the fixed or free edge of a plate." J. Elast., 13, 185-206.

Im, S. (1990). "Asymptotic stress field around a crack normal to the ply-interface of an anisotropic composite laminate." Int. J. Solids Struct., 26(1), 111-127.

Jones, J.P. (1964). "Wave propagation in two-layered medium." J. Appl. Mech., $31,213-222$.

Ju, T.-H., Datta, S.K., and Shah, A.H. (1990). "Scattering of impact waves by a crack in a composite plate." Impact Response and Elastodynamics of Composites (edited by Mal, A.K. and Rajapakse, Y.S.), The American Society of Mechanical Engineers, New York, 153-171.

Kapania, R.K. and Raciti, S. (1989). "Recent advances in analysis of laminated beams and plates, Part II : Vibrations and wave propagation." AIAA Journal, $27(7), 935-946$

Kaul, R.K. and Mindlin, R.D. (1962). "Frequency spectrum of a monoclinic crystal plate." J. Acoust. Soc. Am., 34(12), 1902-1910.

Kinra, V.K., Petraitis, M.S., and Datta, S.K. (1980). "Ultrasonic wave propagation in a random particulate composite." Int. J. Solids and Struct., 16, 301-312.

Koshiba, M., Karakida, S., and Suzuki, M. (1984). "Finite element analysis of Lamb wave scattering in an elastic plate wave guide." IEEE Tran. on Sonics and Ultrasonics, SU-31(1), 18-25.

Ledbetter, H.M. and Datta, S.K. (1984). "Young's modulus and the internal friction of an SiC-particle-reinforced aluminum composite." Matls Sc. and Eng., 67, 2530 .

Ledbetter, H.M. and Datta, S.K. (1986). "Effective wave speed in an SiC-particlereinforced Al composite." J. Acoust. Soc. Am., 79(2), 239-248. 
Ledbetter, H.M., Datta, S.K., and Kyono, T. (1989). "Elastic constants of a graphite-magnesium composite." J. Appl. Phys., 65(9), 3411-3416.

Lee, P.C.Y. and Chang, N. (1979). "Harmonic waves in elastic sandwich plates," J. Elast., 9(1), 51-69.

Lekhnitskii, S.G. (1963). Theory of Elasticity in an Anisotropic Body, Holden Day, San Francisco.

Librescu, L. and Reddy, J.N. (1989). "A few remarks concerning several refined theories of anisotropic composite laminated plates." Int. J. Eng. Sci., 27(5), 515-527.

Mal, A.K. (1988). "Wave propagation in layered composite laminates under periodic surface loads." Wave Motion, 10, 257-266.

Mal, A.K., Xu, P., and Bar-Cohen, Y. (1990). "Leaky Lamb waves for the ultrasonic nondestructive evaluation of adhesive bonds." J. of Eng. Matls. and Tech., 112, 255-259.

Meitzler, A.H. (1965). "Backward-wave transmission of stress pulses in elastic cylinders and plates." J. Acoust. Soc. Am., 38, 835-842.

Mikata, Y. and Achenbach, J.D. (1988). "Interaction of harmonic waves with a periodic array of inclined cracks." Wave Motion, 10, 59-72.

Miklowitz, J. (1960). "Recent developments in elastic wave propagation." Appl. Mech. Rev., 13(12), 865-878.

Mindlin, R.D. (1960). "Waves and vibrations in isotropic, elastic solids." Proc. 1st Symp. Naval Structural Mech., (edited by Goodier, J.N., and Hoff, N.), Pergamon Press, 199-232.

Nayfeh, A.H. and Chimenti, D. (1988). "Ultrasonic wave reflection from liquid- 
coupled orthotropic plates with application to fibrous composite." J. Appl. Mech. $, 55,863-870$.

Nayfeh, A.H., and Chimenti, D.E. (1989). "Free wave propagation in plates of general anisotropic media." J. Appl. Mech., 56, 881-886.

Nishimura, N. and Kobayashi, S. (1991). "A boundary integral equation method for an inverse problem related to crack detection." Int. J. Numer. Meth. Eng., $32,1371-1387$.

Paskaramoorthy, R., Shah, A.H., and Datta, S.K. (1989). "Scattering of flexural waves by a crack in a plate." Eng. Fract. Mech., 33(4), 589-598.

Pilarski, A. and Rose, J.L. (1988). "A transverse-wave ultrasonic oblique incidence technique for interfacial weakness detection in adhesive bonds." J. Appl. Phys., $63(2), 300-307$.

Postma, G.W. (1955). "Wave propagation in a stratified medium." Geophysics., 20, $780-806$.

Press, W.H., Flannery, B.P., Teukolsky, S.A., and Wetterling, W.T. (1988). Numerical Recipes, Cambridge University Press, London.

Read, D.T. and Ledbetter, H.M. (1977). "Elastic properties of a Boron-Aluminum composite at low temperature." J. Appl. Phys., 48(7), 2827-2831.

Rokhlin, S.I. (1980). "Diffraction of Lamb waves by a finite crack in an elastic layer." J. Acoust. Soc. Am., 67(4), 1157-1165.

Rokhlin, S.I. (1981). "Resonance phenomena of Lamb wave scattering by a finite crack in a solid layer." J. Acoust. Soc. Am., 69(4), 922-926.

Rokhlin, S.I. and Marom, D. (1986). "Study of adhesive bonds using low frequency obliquely incident ultrasonic waves." J. Acoust. Soc. Am., 80(2), 585-590. 
Shah, A.H., Wong, K.C., and Datta, S.K. (1982). "Diffraction of plane SH waves in a half-space." Earthquake Eng. Struct. Dyn., 10, 519-528.

Spetzler, H. and Datta, S.K. (1990). "Experimental flaw detection by scattering of plate waves." Elastic Waves and Ultrasonic Nondestructive Evaluation (edited by Datta, S.K., Achenbach, J.D., and Rajapakse, Y.S.), Elsevier Science Publishers, North Holland, 135-141.

Tan, M. and Auld, B.A. (1980). "Normal mode variational method for the twoand three- dimensional accoustic scattering in an isotropic plate." 1980 Ultrason. Symp. Proc., 857-861.

Ting, T.C.T and Hoang, P.H. (1984). "Singularities at the tip of a crack normal to the interface of an anisotropic layered composite." Int. J. Solids Struct., 20(5), 439-454.

Torvik, P.J. (1967). Reflection of wave trains in semi-infinite plates. J. Acoust. Soc. Am., 41(2), 346-353.

Varadan, V.K., Ma, Y., and Varadan, V.V. (1985). "A multiple scattering theory for elastic wave propagation in discrete random media." J. Acoust. Soc. Am., $77(2), 375-385$.

Williams, M.L. (1959). "The stress around a fault or crack in dissimilar media." Bull. Seism. Soc. Am., 49(2), 199-204.

Wong, K.C. (1985). "Elastic wave scattering by arbitrarily-shaped inhomogeneities." Ph.D. Thesis, Dept. of Civil Eng., The University of Manitoba, Winnipeg, Manitoba, Canada.

Wu, C.H. and Plunkett, R. (1967). On the solutions of plates, strips, rods and cylinders subjected to arbitrary dynamic edge load. S.I.A.M. J. Appl. Math., $15,107-119$. 
Yeo, J.K.T. (1983). "Three dimensional analysis of harmonic waves in a periodically laminated medium." M.S. Thesis, Dept. Civil Eng., The University of Manitoba, Winnipeg, Manitoba, Canada.

Yu, Y.Y. (1960). "Forced flexural vibrations of elastic sandwich plate." J. Appl. Mech., 27, 535-540.

Zienkiewicz, O.C. (1977). The Finite Element Method, McGraw-Hill, London. 


\section{Appendix A}

\section{Expressions for Material Property Transformation}

The relations between $D_{i j}$ of equation (2.4) and $C_{I J}$ of equation (2.2) are given by

$$
\begin{aligned}
& D_{11}=C_{11} m^{4}+C_{33} n^{4}+2\left(C_{12}+2 C_{66}\right) m^{2} n^{2}+D_{11}^{*} \\
& D_{12}=\left(C_{11}+C_{22}-4 C_{66}\right) m^{2} n^{2}+C_{12}\left(m^{4}+n^{4}\right)+D_{12}^{*}, \\
& D_{13}=C_{13} m^{2}+C_{23} n^{2}+D_{13}^{*} \\
& D_{16}=\left[\left(C_{12}-C_{11}+2 C_{66}\right) m^{2}+\left(C_{22}-C_{12}-2 C_{66}\right) n^{2}\right] m n+D_{16}^{*}, \\
& D_{22}=C_{11} n^{4}+C_{22} m^{4}+2\left(C_{12}+2 C_{66}\right) m^{2} n^{2}+D_{22}^{*} \\
& D_{23}=C_{13} n^{2}+C_{23} m^{2}+D_{23}^{*} \\
& D_{26}=\left[\left(C_{12}-C_{11}+2 C_{66}\right) n^{2}+\left(C_{22}-C_{12}-2 C_{66}\right) m^{2}\right] m n+D_{26}^{*}, \\
& D_{33}=C_{33}, \\
& D_{36}=\left(C_{23}-C_{13}\right) m n+D_{36}^{*} \\
& D_{44}=C_{44} m^{2}+C_{55} n^{2}+D_{44}^{*} \\
& D_{45}=\left(C_{44}-C_{55}\right) m n+D_{45}^{*} \\
& D_{55}=C_{55} m^{2}+C_{44} n^{2}+D_{55}^{*} \\
& D_{66}=\left(C_{11}+C_{22}-2 C_{12}\right) m^{2} n^{2}+C_{66}\left(m^{2}-n^{2}\right)^{2}+D_{66}^{*}, \\
& D_{1},
\end{aligned}
$$

where $m=\cos \theta, n=\sin \theta$, and $D_{i j}^{*}$ are as defined below.

(i) Transversely isotropic and orthotropic materials

$$
D_{i j}^{*}=0
$$

It should be noted that, for orthotropic materials, equation (2.3) does not hold, and there are no axes of material symmetry. Therefore, $X$ axis is chosen in the direction 
of the normal to one of the material planes of symmetry perpendicular to the plane of the plate. Definition of $Y$ and $Z$ axes remains unchanged.

(ii) Monoclinic materials

$$
\begin{aligned}
& D_{11}^{*}=4\left(C_{16} m^{2}+C_{26} n^{2}\right) m n \\
& D_{12}^{*}=-2\left(C_{16}-C_{26}\right)\left(m^{2}-n^{2}\right) m n \\
& D_{13}^{*}=2 C_{36} m n \\
& D_{16}^{*}=C_{16} m^{2}\left(m^{2}-3 n^{2}\right)+C_{26} n^{2}\left(3 m^{2}-n^{2}\right), \\
& D_{22}^{*}=-4\left(C_{26} m^{2}+C_{16} n^{2}\right) m n \\
& D_{23}^{*}=-2 C_{66} m n \\
& D_{26}^{*}=C_{26} m^{2}\left(m^{2}-3 n^{2}\right)+C_{16} n^{2}\left(3 m^{2}-n^{2}\right), \\
& D_{36}^{*}=C_{36}\left(m^{2}-n^{2}\right), \\
& D_{44}^{*}=-2 C_{55} m n \\
& D_{45}^{*}=C_{45}\left(m^{2}-n^{2}\right), \\
& D_{55}^{*}=2 C_{45} m n \\
& D_{66}^{*}=2\left(C_{26}-C_{16}\right) m n\left(m^{2}-n^{2}\right),
\end{aligned}
$$

For monoclinic materials, equation (2.3) does not hold, and there are no axes of material symmetry. However, there exists only one plane of material symmetry for this material. In writing the material property transformation equations, it has been assumed that the plane of material symmetry is parallel to the plane of the plate. $X$ axis is chosen in an arbitrary direction lying on the plane of the material symmetry, and definition of $Y$ and $Z$ axes remains unchanged. 


\section{Appendix B}

\section{Expressions for Propagator Matrices}

The elements of the propagator matrix $\left[P_{i}\right]$ appearing in equation (2.14) are as given below.

$$
\left[P_{i}\right]=\left[\begin{array}{llll}
p_{11} & p_{12} & p_{13} & p_{14} \\
p_{21} & p_{22} & p_{23} & p_{24} \\
p_{31} & p_{32} & p_{33} & p_{34} \\
p_{41} & p_{42} & p_{43} & p_{44}
\end{array}\right]
$$

where

$$
\begin{aligned}
& p_{11}=\frac{D_{55}}{\Delta_{1}} j k\left[d \cos \left(2 h s_{1}\right)-c B \cos \left(2 h s_{2}\right)\right] \\
& p_{21}=\frac{D_{55}}{\Delta_{1}}\left[-s_{1} A d \sin \left(2 h s_{1}\right)+s_{2} c \sin \left(2 h s_{2}\right)\right], \\
& p_{31}=\frac{D_{55}^{2}}{\Delta_{1}} c d\left[\cos \left(2 h s_{1}\right)-\cos \left(2 h s_{2}\right)\right] \\
& p_{41}=\frac{D_{55}^{2}}{\Delta_{1}} j k\left[-s_{1} d(1+A) \sin \left(2 h s_{1}\right)+s_{2} c(1+B) \sin \left(2 h s_{2}\right)\right], \\
& p_{12}=\frac{D_{55}}{\Delta_{2}} k^{2}\left[-s_{2}(1+B) \sin \left(2 h s_{1}\right)+B s_{1}(1+A) \sin \left(2 h s_{2}\right)\right], \\
& p_{22}=\frac{D_{55}}{\Delta_{2}} j k s_{1} s_{2}\left[A(1+B) \cos \left(2 h s_{1}\right)-(1+A) \cos \left(2 h s_{2}\right)\right] \\
& p_{32}=\frac{D_{55}^{2}}{\Delta_{2}} j k\left[c s_{2}(1+B) \sin \left(2 h s_{1}\right)-d s_{1}(1+A) \sin \left(2 h s_{2}\right)\right], \\
& p_{42}=\frac{D_{55}^{2}}{\Delta_{2}} k^{2} s_{1} s_{2}(1+A)(1+B)\left[-\cos \left(2 h s_{1}\right)+\cos \left(2 h s_{2}\right)\right] \\
& p_{13}=\frac{B k^{2}}{\Delta_{1}}\left[\cos \left(2 h s_{1}\right)-\cos \left(2 h s_{2}\right)\right], \\
& p_{23}=\frac{j k}{\Delta_{1}}\left[s_{1} A B \sin \left(2 h s_{1}\right)-s_{2} \sin \left(2 h s_{2}\right)\right], \\
& p_{33}=\frac{D_{55}}{\Delta_{1}} j k\left[-B c \cos \left(2 h s_{1}\right)+d \cos \left(2 h s_{2}\right)\right], \\
& p_{43}=\frac{D_{55}}{\Delta_{1}} k^{2}\left[-s_{1} B(1+A) \sin \left(2 h s_{1}\right)+s_{2}(1+B) \sin \left(2 h s_{2}\right)\right],
\end{aligned}
$$




$$
\begin{aligned}
p_{14} & =\frac{j k}{\Delta_{2}}\left[-s_{2} \sin \left(2 h s_{1}\right)+A B s_{1} \sin \left(2 h s_{2}\right)\right], \\
p_{24} & =\frac{A}{\Delta_{2}} s_{1} s_{2}\left[-\cos \left(2 h s_{1}\right)+\cos \left(2 h s_{2}\right)\right], \\
p_{34} & =\frac{D_{55}}{\Delta_{2}}\left[-s_{2} c \sin \left(2 h s_{1}\right)+s_{1} A d \sin \left(2 h s_{2}\right)\right], \\
p_{44} & =\frac{D_{55}}{\Delta_{2}} j k s_{1} s_{2}\left[-(1+A) \cos \left(2 h s_{1}\right)+A(1+B) \cos \left(2 h s_{2}\right)\right],
\end{aligned}
$$

in which,

$$
\begin{aligned}
c & =\left(1-\delta_{1}\right) k^{2}-\beta_{1} s_{1}^{2} A, \\
d & =\left(1-\delta_{1}\right) k^{2} B-\beta_{1} s_{2}{ }^{2}, \\
\Delta_{1} & =D_{55} j k \beta_{1} s_{2}{ }^{2}\left(\frac{s_{1}{ }^{2}}{s_{2}{ }^{2}} A B-1\right), \\
\Delta_{2} & =D_{55} j k s_{1} s_{2}(A B-1), \\
h & =\frac{1}{2}\left(z_{i+1}-z_{i}\right) .
\end{aligned}
$$

The propagator matrix $\left[P_{i}^{(o)}\right]$ appearing in equation $(2.41)$ is given by

$$
\left[P_{i}^{(o)}\right]=[E]\left[T_{1}\right][H]
$$

where

$$
\left[T_{1}\right]=\left[\begin{array}{cccccc}
\cos \left(2 h r_{1}\right) & 0 & 0 & \sin \left(2 h r_{1}\right) & 0 & 0 \\
0 & \cos \left(2 h r_{2}\right) & 0 & 0 & \sin \left(2 h r_{2}\right) & 0 \\
0 & 0 & \cos (2 h \zeta) & 0 & 0 & \sin (2 h \zeta) \\
-\sin \left(2 h r_{1}\right) & 0 & 0 & \cos \left(2 h r_{1}\right) & 0 & 0 \\
0 & -\sin \left(2 h r_{2}\right) & 0 & 0 & \cos \left(2 h r_{2}\right) & 0 \\
0 & 0 & -\sin (2 h \zeta) & 0 & 0 & \cos (2 h \zeta)
\end{array}\right]
$$




$$
\begin{aligned}
& {[E]=\left[\begin{array}{cc}
{\left[\left[T_{2}\right][R]\right]} & {[0]} \\
{[0]} & {\left[\left[T_{2}\right][S]\right]}
\end{array}\right]} \\
& {[H]=\left[\begin{array}{cc}
{\left[\left[T_{2}\right][R]\right]^{-1}} & {[0]} \\
{[0]} & {\left[\left[T_{2}\right][S]\right]^{-1}}
\end{array}\right]} \\
& {\left[T_{2}\right]=\left[\begin{array}{ccc}
m & n & 0 \\
-n & m & 0 \\
0 & 0 & 1
\end{array}\right]} \\
& {[S]=\left[\begin{array}{ccc}
C_{55} j K r_{1}\left(1+A^{(o)}\right) & C_{55} j K r_{2}\left(1+B^{(o)}\right) & C_{55} K L \\
2 C_{55} \varepsilon_{2} j L r_{1} A^{(o)} & 2 C_{55} \varepsilon_{2} j L r_{2} & C_{55} \varepsilon_{2}\left(L^{2}-\zeta^{2}\right) \\
r_{1} A^{(o)} & r_{2} & -j L
\end{array}\right],} \\
& {[R]=\left[\begin{array}{ccc}
j K & j K B^{(o)} & 0 \\
j L A^{(o)} & j L & -\zeta \\
R_{31} & R_{32} & 2 C_{55} \varepsilon_{2} j L \zeta
\end{array}\right]} \\
& R_{31}=C_{55}\left[\left(1-\delta_{2}\right) K^{2}-A^{(o)} \beta_{2}\left(r_{1}^{2}+L^{2}\right)+2 A^{(o)} \varepsilon_{2} L^{2}\right] \text {, } \\
& R_{32}=C_{55}\left[\left(1-\delta_{2}\right) K^{2} B^{(o)}-\beta_{2}\left(r_{2}{ }^{2}+L^{2}\right)+2 \varepsilon_{2} L^{2}\right] .
\end{aligned}
$$




\section{Appendix $\mathrm{C}$}

\section{Expressions for Stiffness Matrices}

To define the matrices $\left[c_{2}\right],\left[c_{3}\right],[m]$, and $\left[e_{1}\right]$ through $\left[e_{6}\right]$ appearing in equation (2.56) and $\left[C_{1}\right],\left[C_{2}\right],[M]$, and $\left[E_{1}\right]$ through $\left[E_{5}\right]$ appearing in equation (2.58), the following matrices are defined first:

$$
\begin{aligned}
& {\left[N_{1}\right]=\left[\begin{array}{ll}
{\left[N_{3}\right]} & {\left[N_{4}\right]}
\end{array}\right],} \\
& {\left[N_{2}\right]=\left[\begin{array}{ll}
{\left[N_{5}\right]} & \left.\left[N_{6}\right]\right],
\end{array}\right.} \\
& {[a]=\left[\begin{array}{ll}
{\left[a_{1}\right]} & {\left[a_{2}\right]}
\end{array}\right],} \\
& {[b]=\left[\left[\begin{array}{ll}
{\left[b_{1}\right]} & {\left[b_{2}\right.}
\end{array}\right],\right.} \\
& {[d]=\left[\begin{array}{ll}
{\left[d_{1}\right]} & \left.\left[d_{2}\right]\right],
\end{array}\right.} \\
& {\left[N_{3}\right]=\left[\begin{array}{cccccc}
0 & 0 & 0 & 0 & -n_{3} & 0 \\
0 & 0 & 0 & 0 & 0 & 0 \\
\frac{-n_{3} D_{13}}{D_{33}} & 0 & \frac{-n_{3} D_{36}}{D_{33}} & 0 & 0 & 0
\end{array}\right],} \\
& {\left[N_{5}\right]=\left[\begin{array}{cccccc}
n_{1} & \frac{n_{3} D_{44}}{\Delta} & 0 & \frac{-n_{3} D_{45}}{\Delta} \Delta_{55} & 0 & 0 \\
0 & \frac{-n_{3} D_{45}}{\Delta} & n_{1} & \frac{n_{3} D_{55}}{\Delta} & 0 & 0 \\
0 & 0 & 0 & 0 & n_{1} & \frac{n_{3}}{D_{33}}
\end{array}\right]} \\
& {\left[a_{1}\right]=\left[\begin{array}{cccccc}
0 & 0 & 0 & 0 & 0 & 0 \\
0 & 0 & 0 & 0 & n_{1}^{\prime} & \frac{n_{3}^{\prime}}{D_{33}} \\
0 & \frac{-n_{3}^{\prime} D_{45}}{\Delta} & n_{1}^{\prime} & \frac{n_{3}^{\prime} D_{55}}{\Delta} & 0 & 0 \\
n_{1}^{\prime} & \frac{n_{3}^{\prime} D_{44}}{\Delta} & 0 & \frac{-n_{3}^{\prime} D_{45}}{\Delta} & 0 & 0 \\
0 & 0 & 0 & 0 & 0 & 0
\end{array}\right],}
\end{aligned}
$$




$$
\begin{aligned}
& {\left[b_{1}\right]=\left[\begin{array}{cccccc}
n_{1} & \frac{n_{3} D_{44}}{\Delta} & 0 & \frac{-n_{3} D_{45}}{\Delta} & 0 & 0 \\
\frac{-n_{3}^{\prime} D_{13}}{D_{33}} & 0 & \frac{-n_{3}^{\prime} D_{36}}{D_{33}} & 0 & 0 & 0 \\
0 & 0 & 0 & 0 & 0 & 0 \\
0 & 0 & 0 & 0 & n_{1}-n_{3}^{\prime} & \frac{n_{3}}{D_{33}} \\
0 & \frac{-n_{3} D_{45}}{\Delta} & n_{1} & \frac{n_{3} D_{55}}{\Delta} & 0 & 0
\end{array}\right]} \\
& {\left[d_{1}\right]=\left[\begin{array}{cccccc}
0 & 0 & 0 & 0 & -n_{3} & 0 \\
0 & 0 & 0 & 0 & 0 & 0 \\
0 & 0 & 0 & 0 & 0 & 0 \\
\frac{-n_{3} D_{13}}{D_{33}} & 0 & \frac{-n_{3} D_{36}}{D_{33}} & 0 & 0 & 0 \\
0 & 0 & 0 & 0 & 0 & 0
\end{array}\right] .}
\end{aligned}
$$

The matrices $\left[N_{4}\right],\left[N_{6}\right],\left[a_{2}\right],\left[b_{2}\right]$, and $\left[d_{2}\right]$, are obtained from $\left[N_{3}\right],\left[N_{5}\right],\left[a_{1}\right],\left[b_{1}\right]$, and $\left[d_{1}\right]$, respectively, after replacing $n_{1}$ by $n_{2}, n_{3}$ by $n_{4}, n_{1}^{\prime}$ by $n_{2}^{\prime}$ and $n_{3}^{\prime}$ by $n_{4}^{\prime}$.

Then

$$
\begin{aligned}
& {\left[c_{2}\right]=\int_{-h}^{h} \rho\left[N_{1}\right]^{\mathrm{T}}\left[N_{1}\right] d z,} \\
& {\left[c_{3}\right]=\int_{-h}^{h} \rho\left[N_{1}\right]^{\mathrm{T}}\left[N_{2}\right] d z,} \\
& {[m]=\int_{-h}^{h} \rho\left[N_{2}\right]^{\mathrm{T}}\left[N_{2}\right] d z,} \\
& {\left[e_{1}\right]=\int_{-h}^{h}[d]^{\mathrm{T}}[D][d] d z,} \\
& {\left[e_{2}\right]=\int_{-h}^{h}[d]^{\mathrm{T}}[D][b] d z,} \\
& {\left[e_{3}\right]=\int_{-h}^{h}[d]^{\mathrm{T}}[D][a] d z,} \\
& {\left[e_{4}\right]=\int_{-h}^{h}[b]^{\mathrm{T}}[D][b] d z,} \\
& {\left[e_{5}\right]=\int_{-h}^{h}[b]^{\mathrm{T}}[D][a] d z,} \\
& {\left[e_{6}\right]=\int_{-h}^{h}[a]^{\mathrm{T}}[D][a] d z,}
\end{aligned}
$$




$$
\begin{aligned}
& {\left[C_{1}\right]=\cup\left[\left[c_{3}\right]-\left[c_{3}\right]^{\mathrm{T}}\right],} \\
& {\left[C_{2}\right]=\cup\left[\left[c_{2}\right]\right],} \\
& {\left[E_{1}\right]=\cup\left[\left[e_{1}\right]\right],} \\
& {\left[E_{2}\right]=\cup\left[\left[e_{2}\right]-\left[e_{2}\right]^{\mathrm{T}}\right],} \\
& {\left[E_{3}\right]=\cup\left[\left[e_{3}\right]-\left[e_{4}\right]+\left[e_{3}\right]^{\mathrm{T}}\right],} \\
& {\left[E_{4}\right]=\cup\left[\left[e_{5}\right]^{\mathrm{T}}-\left[e_{5}\right]\right],} \\
& {\left[E_{5}\right]=\cup\left[\left[e_{6}\right]\right],} \\
& {[M]=\cup[[m]],}
\end{aligned}
$$

where $U$ stands for union or assembly. 


\section{Appendix D}

\section{Derivation of the Reciprocity Relations}

The reciprocity relations given in equations (4.36), and the reciprocity relations for general case are derived in this appendix.

The reciprocity relations are derived from the elastodynamic reciprocity theorem (Achenbach, 1973; Auld, 1973; Tan and Auld, 1980) which may be written, in the absence of body forces, in the following form using tensor notation:

$$
\oint_{S}\left(u_{j}^{\mathrm{B}} \sigma_{j k}^{\mathrm{A}}-u_{j}^{\mathrm{A}} \sigma_{j k}^{\mathrm{B}}\right) n_{k} d S=0, \quad j, k=x, y, z
$$

where $u_{j}^{\mathrm{A}}$ and $\sigma_{j k}^{\mathrm{A}}$ represent the displacements and stresses corresponding to elas-

todymanic state A while $u_{j}^{\mathrm{B}}$ and $\sigma_{j k}^{\mathrm{B}}$ are the displacements and stresses corresponding to elastodynamic state $\mathrm{B}$, in a region $\mathrm{V}$ bounded by a surface $\mathrm{S}$. Wave fields corresponding to both elastodynamic states vary harmonically in time with circular frequency $\omega$. In writing equation (D.1), Einstein's summation convention of repeated indices has been assumed to hold, and the displacement components $u, v, w$ have been represented by $u_{x}, u_{y}, u_{z}$, respectively. In the ensuing $\S \mathrm{D} .1$, derivation of reciprocity relations for plane strain case is given. General case is treated in §D.2.

\section{D.1 Plane Strain Case}

In order to derive the reciprocity relations, the orthogonality relations among wave modes have to be established first.

\section{D.1.1 Orthogonality Relations}

The orthogonality relations can be derived by applying the reciprocity theorem 
for two different wave modes travelling in an infinite laminated composite plate with no inhomogeneity.

Let $\mathrm{V}$ be the region bounded by the planar surfaces $x=x_{1}, x=x_{2}, z=0$, and $z=H$. Herein, $x_{1}$ and $x_{2}$ are any two arbitray $x$ coordinates chosen in such a way that $x_{2}>x_{1}$. The state $\mathrm{A}$ is taken to be the field due to $-n$-th wave mode (namely, the field due to the wave travelling in the negative $x$ direction with wave number $k_{n}$ ), and the state B is chosen as the field due to $m$-th wave mode (namely, the field due to the wave travelling in the positive $x$ direction with wave number $\left.k_{m}\right)$. The wave fields corresponging to two states can be written as

$$
\begin{gathered}
u_{j}^{\mathrm{A}} \rightarrow A_{-n}\left\{u_{-n}\right\} \exp \left(-j k_{n} x\right), \\
\sigma_{j k}^{\mathrm{A}} \rightarrow A_{-n}\left\{\sigma_{-n}\right\} \exp \left(-j k_{n} x\right), \\
u_{j}^{\mathrm{B}} \rightarrow A_{m}\left\{u_{m}\right\} \exp \left(j k_{m} x\right), \\
\sigma_{j k}^{\mathrm{B}} \rightarrow A_{m}\left\{\sigma_{m}\right\} \exp \left(j k_{m} x\right),
\end{gathered}
$$

where $A_{m},\left\{u_{m}\right\}$, and $\left\{\sigma_{m}\right\}$ represent the amplitude, displacement mode shape vector, and the mode shape vector of tractions on $x$ face, respectively, for the $m$ th wave mode; and $A_{-n},\left\{u_{-n}\right\}$, and $\left\{\sigma_{-n}\right\}$ represent the same quantities for the $-n$-th wave mode. Application of the reciprocity theorem given in equation (D.1) to the region $\mathrm{V}$ for the states $\mathrm{A}$ and $\mathrm{B}$ defined in equations (D.2) results in

$$
A_{m} A_{-n}\left\{\exp \left[j\left(k_{m}-k_{n}\right) x_{2}\right]-\exp \left[j\left(k_{m}-k_{n}\right) x_{1}\right]\right\} I_{1}=0
$$

where

$$
I_{1}=\int_{0}^{H}\left(\left\{u_{m}\right\}^{\mathrm{T}}\left\{\sigma_{-n}\right\}-\left\{u_{-n}\right\}^{\mathrm{T}}\left\{\sigma_{m}\right\}\right) d z
$$

Since $x_{1}$ and $x_{2}$ are arbitrary, equation (D.3) leads to the orthogonality relation

$$
\int_{0}^{H}\left(\left\{u_{m}\right\}^{\mathrm{T}}\left\{\sigma_{-n}\right\}-\left\{u_{-n}\right\}^{\mathrm{T}}\left\{\sigma_{m}\right\}\right) d z=0, \quad \text { for } m \neq n .
$$


In a similar manner, the following orthogonality relations can be derived:

$$
\begin{array}{r}
\int_{0}^{H}\left(\left\{u_{m}\right\}^{\mathrm{T}}\left\{\sigma_{n}\right\}-\left\{u_{n}\right\}^{\mathrm{T}}\left\{\sigma_{m}\right\}\right) d z=0 ; \\
\int_{0}^{H}\left(\left\{u_{-m}\right\}^{\mathrm{T}}\left\{\sigma_{-n}\right\}-\left\{u_{-n}\right\}^{\mathrm{T}}\left\{\sigma_{-m}\right\}\right) d z=0 .
\end{array}
$$

\section{D.1.2 Reciprocity Realtions}

With a view to derive the reciprocity relations among conversion coefficients, the plate with the inhomogeneity is considered next. Choosing $\mathrm{V}$ as the region of the plate, bounded by the palnar surfaces $z=0, z=H, x=x_{3}$, and $x=x_{4}$ $\left(x_{3} \geq x^{+}, x_{4} \leq x^{-}\right)$; state $\mathrm{A}$ as the total field (incident plus scattered) due to $\boldsymbol{n}$-th incident wave mode; and state $B$ as the total field due to $-p$-th incident wave mode, equation (D.1) can be written as

$$
I_{2}-I_{3}=0
$$

where

$$
\begin{aligned}
& I_{2}=\left.\int_{0}^{H}\left(\left\{u^{\mathrm{B}}\right\}^{\mathrm{T}}\left\{\sigma^{\mathrm{A}}\right\}-\left\{u^{\mathrm{A}}\right\}^{\mathrm{T}}\left\{\sigma^{\mathrm{B}}\right\}\right)\right|_{x_{3}} d z \\
& I_{3}=\left.\int_{0}^{H}\left(\left\{u^{\mathrm{B}}\right\}^{\mathrm{T}}\left\{\sigma^{\mathrm{A}}\right\}-\left\{u^{\mathrm{A}}\right\}^{\mathrm{T}}\left\{\sigma^{\mathrm{B}}\right\}\right)\right|_{x_{4}} d z .
\end{aligned}
$$

The total wave field at $x_{3}$ is given by

$$
\begin{aligned}
& \left.\left\{u^{\mathrm{A}}\right\}\right|_{x_{3}}=A_{-n}\left[\left\{u_{-n}\right\} \exp \left(-j k_{n} x_{3}\right)+\sum_{q=1}^{M} R_{n q}\left\{u_{q}\right\} \exp \left(j k_{q} x_{3}\right)\right] \\
& \left.\left\{\sigma^{\mathrm{A}}\right\}\right|_{x_{3}}=A_{-n}\left[\left\{\sigma_{-n}\right\} \exp \left(-j k_{n} x_{3}\right)+\sum_{q=1}^{M} R_{n q}\left\{\sigma_{q}\right\} \exp \left(j k_{q} x_{3}\right)\right] \\
& \left.\left\{u^{\mathrm{B}}\right\}\right|_{x_{3}}=A_{-p}\left[\left\{u_{-p}\right\} \exp \left(-j k_{p} x_{3}\right)+\sum_{m=1}^{M} R_{p m}\left\{u_{m}\right\} \exp \left(j k_{m} x_{3}\right)\right], \\
& \left.\left\{\sigma^{\mathrm{B}}\right\}\right|_{x_{3}}=A_{-p}\left[\left\{\sigma_{-p}\right\} \exp \left(-j k_{p} x_{3}\right)+\sum_{m=1}^{M} R_{p m}\left\{\sigma_{m}\right\} \exp \left(j k_{m} x_{3}\right)\right]
\end{aligned}
$$


where $R_{n q}$ and $R_{p m}$ represent reflection coefficients. The total wave field at $x_{4}$ can be written as

$$
\begin{aligned}
& \left.\left\{u^{\mathrm{A}}\right\}\right|_{x_{4}}=A_{-n} \sum_{q=1}^{M} T_{n q}\left\{u_{-q}\right\} \exp \left(-j k_{q} x_{4}\right), \\
& \left.\left\{\sigma^{\mathrm{A}}\right\}\right|_{x_{4}}=A_{-n} \sum_{q=1}^{M} T_{n q}\left\{\sigma_{-q}\right\} \exp \left(-j k_{q} x_{4}\right), \\
& \left.\left\{u^{\mathrm{B}}\right\}\right|_{x_{4}}=A_{-p} \sum_{m=1}^{M} T_{p m}\left\{u_{-m}\right\} \exp \left(-j k_{m} x_{4}\right), \\
& \left.\left\{\sigma^{\mathrm{B}}\right\}\right|_{x_{4}}=A_{-p} \sum_{m=1}^{M} T_{p m}\left\{\sigma_{-m}\right\} \exp \left(-j k_{m} x_{4}\right),
\end{aligned}
$$

where $T_{n q}$ and $T_{p m}$ represent transmission coefficients. Substitution of equations (D.10) in equation (D.9a), and subsequent simplification of the resulting equation by making use of the orthogonality relations given in equations (D.5)-(D.7) leads to

$$
I_{2}=A_{-p} A_{-n}\left(R_{p n} \varsigma_{n}-R_{n p} \varsigma_{p}\right)
$$

where $\varsigma_{p}$ and $\varsigma_{n}$ are given by

$$
\varsigma_{m}=\int_{0}^{H}\left(\left\{u_{m}\right\}^{\mathrm{T}}\left\{\sigma_{-m}\right\}-\left\{u_{-m}\right\}^{\mathrm{T}}\left\{\sigma_{m}\right\}\right) d z, \quad m=p, n .
$$

In view of the orthogonality relations, substituion of equations (D.11) into equation (D.9b) results in

$$
I_{3}=0
$$

Equations (D.8), (D.12), and (D.14) leads to

$$
R_{p n} \varsigma_{n}=R_{n p} \varsigma_{p}
$$

which is the reciprocity relation given in equation (4.36a). 
The vectors $\left\{u_{m}\right\}$ and $\left\{\sigma_{m}\right\}$ can be written in the component form as

$$
\begin{aligned}
& \left\{u_{m}\right\}=\left\{\begin{array}{l}
u_{x m} \\
u_{z m}
\end{array}\right\}, \\
& \left\{\sigma_{m}\right\}=\left\{\begin{array}{l}
\sigma_{x x m} \\
\sigma_{z x m}
\end{array}\right\} .
\end{aligned}
$$

Imagining a wave propagating in the negative $x$ direction as being the mirror image about the plane $x=0$ of a wave propagating in the positive $x$ direction, one finds that $\left\{u_{-m}\right\}$ and $\left\{\sigma_{-m}\right\}$ can be written as

$$
\begin{aligned}
& \left\{u_{-m}\right\}=\left\{\begin{array}{r}
-u_{x m} \\
u_{z m}
\end{array}\right\}, \\
& \left\{\sigma_{-m}\right\}=\left\{\begin{array}{r}
\sigma_{x x m} \\
-\sigma_{z x m}
\end{array}\right\} .
\end{aligned}
$$

In view of equations (D.16) and (D.17), $\varsigma_{m}$ appearing in equation (D.13) take the form

$$
\varsigma_{m}=2 \int_{0}^{H}\left\{u_{m}\right\}^{\mathrm{T}}\left\{\sigma_{-m}\right\} d z
$$

Approximating the integral in equation (D.18), $\varsigma_{m}$ can be written as

$$
\varsigma_{m} \approx-2\left\{F_{m}^{-}\right\}^{\mathrm{T}}\left\{q_{m}\right\}
$$

where $\left\{F_{m}^{-}\right\}$is the $m$-th column of $\left[F^{-}\right]$and $\left\{q_{m}\right\}$ is the $m$-th column of $\left[G^{+}\right]$.

The reciprocity relation given in equation $(4.36 \mathrm{~b})$ can be derived in a similar manner by applying the reciprocity theorem to the same region $\mathrm{V}$, with state $\mathrm{A}$ as the total field due to $n$-th incident mode and state B as the total field due to $-p$-th incident mode. Note that when deriving equation (4.36b), it has been assumed that the geometry of the inhomogeneity is symmetric with respect to the plane $x=0$. 


\section{D.2 General Case}

As in the plane strain case, the first step is to derive the orthogonality relations among wave modes that exist in an infinite plate with no inhomogeneity.

\section{D.2.1 Orthogonality Relations}

For convenience in derivation of orthogonality relations, few notations are introduced first. Let the wavenumber pair $\left(k_{n}, \zeta_{0}\right)$ denotes a wave mode propagating in the first quadrant of $x y$ plane. Herein, $k_{n}$ represents the positive $x$ direction wavenumber, and $\zeta_{0}$ (which is fixed) represents the positive $y$ direction wavenumber as opposed to the definition of $\zeta_{0}$ in Chapter 5. In a similar manner, let the wavenumber pairs $\left(-k_{n}^{*}, \zeta_{0}\right),\left(-k_{n},-\zeta_{0}\right)$, and $\left(k_{n}^{*},-\zeta_{0}\right)$ denote the wave modes corresponding to second, third, and fourth quadrants of $x y$ plane, respectively. It should be mentioned here that if $\left(k_{n}, \zeta_{0}\right)$-th wave mode is an admissible wave mode (of the dispersion relation of the plate), then $\left(-k_{n},-\zeta_{0}\right)$-th wave mode, which is propagating in the opposite direction, is also an admissible wave mode. Similary, if $\left(k_{n}^{*},-\zeta_{0}\right)$-th wave mode is admissible, then $\left(-k_{n}^{*}, \zeta_{0}\right)$-th wave mode is also admissible. This point can be explained by visualising the configuration of the plate with respect to a new coordinate system that is obtained after rotating the $x, y$ axes by $180^{\circ}$ about the $z$ axis. However, when $\left(k_{n}, \zeta_{0}\right)$-th wave mode is an admissible mode, then $\left(k_{n},-\zeta_{0}\right)$-th wave mode is not necessarily an admissible mode. Due to this reason, a superscript star $\left({ }^{*}\right)$ has been introduced to the $x$ direction wavenumbers of wave modes corresponding to second and fourth quadrants of the $x y$ plane. Note that $\left(k_{n},-\zeta_{0}\right)$-th wave mode is admissible when the fibers in each layer are aligned either in $x$ or $y$ directions. This can be visualized by considering the mirror image of the plate with respect to the $x z$ plane. 
The orthogonality relations are derived by applying the reciprocity theorem to the close region $\mathrm{V}$ bounded by the planes $z=0, z=H, x=x_{1}, x=x_{2}, y=y_{1}$, and $y=y_{2}$ where $x_{1}, x_{2}, y_{1}$, and $y_{2}$ are arbitrary coordinates chosen in such a way that $x_{2}>x_{1}$ and $y_{2}>y_{1}$. State $\mathrm{A}$ is taken to be the field due to $\left(-k_{n}^{*}, \zeta_{0}\right)$-th wave mode and state $\mathrm{B}$ is taken as the field due to $\left(k_{m}^{*},-\zeta_{0}\right)$-th wave mode. Then, the wave fields due to two states can be written as

$$
\begin{aligned}
& u_{j}^{\mathrm{A}} \rightarrow A_{-n}^{*+}\left\{u_{-n}^{*+}\right\} \exp \left[j\left(-k_{n}^{*} x+\zeta_{0} y\right)\right], \\
& \sigma_{j k}^{\mathrm{A}} \rightarrow A_{-n}^{*+}\left\{\begin{array}{l}
\left\{\sigma_{-n x}^{*+}\right\} \\
\left\{\sigma_{-n y}^{*+}\right\}
\end{array}\right\} \exp \left[j\left(-k_{n}^{*} x+\zeta_{0} y\right)\right], \\
& u_{j}^{\mathrm{B}} \rightarrow A_{m}^{*-}\left\{u_{m}^{*-}\right\} \exp \left[j\left(k_{m}^{*} x-\zeta_{0} y\right)\right], \\
& \sigma_{j k}^{\mathrm{B}} \rightarrow A_{m}^{*-}\left\{\begin{array}{l}
\left\{\sigma_{m x}^{*-}\right\} \\
\left\{\sigma_{m y}^{*-}\right.
\end{array}\right\} \exp \left[j\left(k_{m}^{*} x-\zeta_{0} y\right)\right]
\end{aligned}
$$

where $A_{-n}^{*+},\left\{u_{-n}^{*+}\right\},\left\{\sigma_{-n x}^{*+}\right\}$, and $\left\{\sigma_{-n y}^{*+}\right\}$ represent the amplitude, displacement mode shape vector, the mode shape vector of tractions on $x$ face, and the mode shape vector of tractions on $y$ face, respectively, for the $\left(-k_{n}^{*}, \zeta_{0}\right)$-th wave mode; and $A_{m}^{*-},\left\{u_{m}^{*-}\right\},\left\{\sigma_{m x}^{*-}\right\}$, and $\left\{\sigma_{m y}^{*-}\right\}$ represent the same quantities for the $\left(k_{m}^{*},-\zeta_{0}\right)$-th wave mode. Application of the reciprocity theorem to the region $\mathrm{V}$ for the states $\mathrm{A}$ and B defined in equations (D.20) results in

$$
\left\{\exp \left[j\left(k_{m}^{*}-k_{n}^{*}\right) x_{2}\right]-\exp \left[j\left(k_{m}^{*}-k_{n}^{*}\right) x_{1}\right]\right\} I_{4}\left[\left(k_{m}^{*},-\zeta_{0}\right) ;\left(-k_{n}^{*}, \zeta_{0}\right)\right]=0
$$

where the notation $I_{4}\left[\left(k_{m}^{*},-\zeta_{0}\right) ;\left(-k_{n}^{*}, \zeta_{0}\right)\right]$ has been used to represent the integral

$$
\int_{0}^{H}\left(\left\{u_{m}^{*-}\right\}^{\mathrm{T}}\left\{\sigma_{-n x}^{*+}\right\}-\left\{u_{-n}^{*+}\right\}^{\mathrm{T}}\left\{\sigma_{m x}^{*-}\right\}\right) d z
$$

Since $x_{1}$ and $x_{2}$ are arbitrary, equation (D.21) leads to the orthogonality relation

$$
I_{4}\left[\left(k_{m}^{*},-\zeta_{0}\right) ;\left(-k_{n}^{*}, \zeta_{0}\right)\right]=0 \quad \text { for } \quad k_{m}^{*} \neq k_{n}^{*} .
$$


It needs to be mentioned here that the net contributions from the surface integrals (see equation (D.1)) on planes $y=y_{1}$ and $y=y_{2}$ amount to zero.

In a similar manner, choosing state $\mathrm{A}$ and $\mathrm{B}$ as the fields due to $\left(k_{n}, \zeta_{0}\right)$-th and $\left(-k_{m},-\zeta_{0}\right)$-th wave modes, respectively, it can be shown that

$$
I_{4}\left[\left(-k_{m},-\zeta_{0}\right) ;\left(k_{n}, \zeta_{0}\right)\right]=0 \quad \text { for } \quad k_{m} \neq k_{n} .
$$

Adopting a similar approach, the following orthogonality relations can be derived:

$$
\begin{array}{r}
I_{4}\left[\left(k_{m}^{*},-\zeta_{0}\right) ;\left(k_{n}, \zeta_{0}\right)\right]=0, \\
I_{4}\left[\left(-k_{m},-\zeta_{0}\right) ;\left(-k_{n}^{*}, \zeta_{0}\right)\right]=0,
\end{array}
$$

In deriving equation (D.24), state $\mathrm{A}$ is chosen as the field due to $\left(k_{n}, \zeta_{0}\right)$-th wave mode and state $\mathrm{B}$ is taken as the field due to $\left(k_{m}^{*},-\zeta_{0}\right)$-th wave mode, whereas in deriving equation (D.25), state $\mathrm{A}$ is chosen as the field due to $\left(-k_{n}^{*}, \zeta_{0}\right)$-th wave mode and state $\mathrm{B}$ is taken as the field due to $\left(-k_{m},-\zeta_{0}\right)$-th wave mode.

\section{D.2.2 Reciprocity Relations}

In order to derive the reciprocity relations, $\mathrm{V}$ is chosen as the region of the plate surrounding the inhomogeneity, bounded by the planar surfaces $z=0, z=H$, $x=x_{3}\left(x_{3} \geq x^{+}\right), x=x_{4}\left(x_{4} \leq x^{-}\right), y=y_{1}$, and $y=y_{2}$ (where $y_{1}$ and $y_{2}$ are arbitrary, and $y_{2}>y_{1}$ ). The reciprocity relations among reflection coeffcients can be derived by choosing state $\mathrm{A}$ as the total field due to $\left(-k_{n},-\zeta_{0}\right)$-th incident wave mode, and state $\mathrm{B}$ as the total field due to $\left(-k_{p}^{*}, \zeta_{0}\right)$-th incident wave mode. Let $R_{n q}$ and $T_{n q}$ denote the reflection and transmission coefficients, respectively, due to $\left(-k_{n},-\zeta_{0}\right)$-th incident wave mode; and $R_{p m}^{*}$ and $T_{p m}^{*}$ denote the same quantities 
due to $\left(-k_{p}^{*}, \zeta_{0}\right)$-th incident wave mode. In view of the orthogonality relations given in equations (D.22)-(D.25), and omitting details of algebra, application of the reciprocity theorem to the region $\mathrm{V}$ results in the reciprocity relation

$$
R_{p n}^{*} \varsigma_{n}^{(g)}=R_{n p} \varsigma_{p}^{(g) *}
$$

where

$$
\begin{aligned}
\varsigma_{n}^{(g)} & =\int_{0}^{H}\left(\left\{u_{n}^{+}\right\}^{\mathrm{T}}\left\{\sigma_{-n x}^{-}\right\}-\left\{u_{-n}^{-}\right\}^{\mathrm{T}}\left\{\sigma_{n x}^{+}\right\}\right) d z, \\
\varsigma_{p}^{(g) *} & =\int_{0}^{H}\left(\left\{u_{p}^{*-}\right\}^{\mathrm{T}}\left\{\sigma_{-p x}^{*-}\right\}-\left\{u_{-p}^{*+}\right\}^{\mathrm{T}}\left\{\sigma_{p x}^{*-}\right\}\right) d z .
\end{aligned}
$$

In equations (D.27), $\left\{u_{n}^{+}\right\},\left\{u_{-n}^{-}\right\},\left\{u_{p}^{*-}\right\}$, and $\left\{u_{-p}^{*+}\right\}$ denote the displacement mode shape vectors corresponding to $\left(k_{n}, \zeta_{0}\right)$-th, $\left(-k_{n},-\zeta_{0}\right)$-th, $\left(k_{p}^{*},-\zeta_{0}\right)$-th, and $\left(-k_{p}^{*}, \zeta_{0}\right)$-th wave modes, respectively, in an infinite plate with no inhomogeneity. The corresponding traction mode shape vectors on $x$ face are denoted by $\left\{\sigma_{n x}^{+}\right\}$, $\left\{\sigma_{-n x}^{-}\right\},\left\{\sigma_{p x}^{*-}\right\}$, and $\left\{\sigma_{-p x}^{*+}\right\}$. It should be noted that the net contribution to the surface integral in equation (D.1) from surfaces $y=y_{1}$ and $y=y_{2}$ becomes zero.

With a view to approximate the integrals in equations (D.27) to a simple form, $\left\{u_{n}^{+}\right\}$and $\left\{\sigma_{n x}^{+}\right\}$are written in the component forms as

$$
\begin{gathered}
\left\{u_{n}^{+}\right\}=\left\{\begin{array}{l}
u_{x n}^{+} \\
u_{y n}^{+} \\
u_{z n}^{+}
\end{array}\right\}, \\
\left\{\sigma_{n x}^{+}\right\}=\left\{\begin{array}{l}
\sigma_{x x n}^{+} \\
\sigma_{x y n}^{+} \\
\sigma_{x z n}^{+}
\end{array}\right\} .
\end{gathered}
$$

Then, it can be shown that $\left\{u_{-n}^{-}\right\}$and $\left\{\sigma_{-n x}^{-}\right\}$are given by

$$
\begin{gathered}
\left\{u_{-n}^{-}\right\}=\left\{\begin{array}{c}
-u_{x n}^{+} \\
-u_{y n}^{+} \\
u_{z n}^{+}
\end{array}\right\}, \\
\left\{\sigma_{-n x}^{-}\right\}=\left\{\begin{array}{c}
\sigma_{x x n}^{+} \\
\sigma_{x y n}^{+} \\
-\sigma_{z x n}^{+}
\end{array}\right\} .
\end{gathered}
$$


In view of equations (D.28) and (D.29), $\varsigma_{n}^{(g)}$ appearing in equation (D.27a) can be written as

$$
\varsigma_{n}^{(g)}=2 \int_{0}^{H}\left\{u_{n}^{+}\right\}^{\mathrm{T}}\left\{\sigma_{-n x}^{-}\right\} d z .
$$

Approximating the integral in equation (D.30), $\varsigma_{n}^{(g)}$ can be written as

$$
\varsigma_{n}^{(g)} \approx-2\left\{F_{n}^{(g)-}\right\}^{\mathrm{T}}\left\{q_{n}^{(g)}\right\}
$$

where $\left\{F_{n}^{(g)-}\right\}$ is the $n$-th column of $\left[F^{(g)-}\right]$, and $\left\{q_{n}^{(g)}\right\}$ is the $n$-th column of $\left[G^{+}\right]$ for general case. $\varsigma_{p}^{(g) *}$ can also be approximated by a form similar to that given in equation (D.30).

Applying the reciprocity theorem to the same region $\mathrm{V}$, with state $\mathrm{A}$ as the total field due to $\left(k_{n}, \zeta_{0}\right)$-th incident mode and state $\mathrm{B}$ as the total field due to $\left(-k_{p},-\zeta_{0}\right)$ th incident mode, the reciprocity relation among transmission coefficients can be shown to have the form

$$
T_{p n} \varsigma_{n}^{(g)}=T_{n p} \varsigma_{p}^{(g)}
$$

where $\varsigma_{p}^{(g)}$ is given by equation (D.31) with $n$ replaced by $p$. As in the plane strain case, when deriving equation (D.32) it has been assumed that the geometry of the inhomogeneity is symmetric with respect to the plane $x=0$.

When the fibers in each layer are either in $x$ or $y$ direction, the the reciprocity relation in equation (D.26) degenerates into

$$
R_{p n} \varsigma_{n}^{(g)}=R_{n p} \varsigma_{p}^{(g)}
$$

With equation (D.26) in hand, following the procedure adopted to derive equation (4.39) in plane strain case, the reciprocity relation among proportions of re- 
flected energies can be derived as

$$
E_{p n}^{+*}=E_{n p}^{+*} .
$$

When the fibers in each layer are aligned in either $x$ or $y$ direction, equation (D.34) degenerates to

$$
E_{p n}^{+}=E_{n p}^{+} .
$$




\section{Appendix $\mathrm{E}$}

\section{Definition of Matrices $\left[T_{3}\right]$ and $\left[T_{4}^{(g)}\right]$}

The matrix $\left[T_{3}\right]$ appearing in equation (4.56) is given by

$$
\left[T_{3}\right]=C_{55}\left[\begin{array}{ccc}
m^{2} & n^{2} & 2 m n \\
-m n & m n & m^{2}-n^{2}
\end{array}\right]
$$

The matrix $\left[T_{4}^{(g)}\right]$ in equation (4.56) is obtained from $\left[T_{4}(K, L)\right]$ after replacing $K$ by $K^{(g)}$, and $L$ by $L^{(g)}$, where $\left[T_{4}(K, L)\right]$ is given by

$$
\left[T_{4}(K, L)\right]=\left[\begin{array}{lll}
t_{11} & t_{12} & t_{13} \\
t_{21} & t_{22} & t_{23} \\
t_{31} & t_{32} & t_{33}
\end{array}\right]
$$

where

$$
\begin{aligned}
& t_{11}=-\lambda_{2} K^{2}+\left(1-\delta_{2}\right)\left(r_{1}{ }^{2}+L^{2}\right) \\
& t_{12}=-\lambda_{2} K^{2} B^{(o)}+\left(1-\delta_{2}\right)\left(r_{2}{ }^{2}+L^{2}\right) \\
& t_{13}=0 \\
& t_{21}=\left(1-\delta_{2}\right) K^{2}-\left[\beta_{2}\left({r_{1}}^{2}+L^{2}\right)-2 \varepsilon_{2} r_{1}{ }^{2}\right] A^{(o)} \\
& t_{22}=\left(1-\delta_{2}\right) K^{2} B^{(o)}-\left[\beta_{2}\left(r_{2}{ }^{2}+L^{2}\right)-2 \varepsilon_{2} r_{2}{ }^{2}\right] \\
& t_{23}=-2 \varepsilon_{2} j L \zeta \\
& t_{31}=-K L\left(1+A^{(o)}\right) \\
& t_{32}=-K L\left(1+B^{(o)}\right) \\
& t_{33}=-j K \zeta .
\end{aligned}
$$




\section{Appendix $\mathrm{F}$}

\section{Expressions for Force Components}

The force components $F_{i m}^{(g) x}, F_{i m}^{(g) y}$, and $F_{i m}^{(g) z}$ appearing in equation (4.58) are given below.

$$
\begin{aligned}
& F_{1 m}^{(g) x}= \frac{h_{1}}{6}\left(2 \hat{\sigma}_{x x 1 m}^{(+)}+\hat{\sigma}_{x x 2 m}^{(-)}\right) \\
& F_{1 m}^{(g) y}= \frac{h_{1}}{6}\left(2 \hat{\sigma}_{x y 1 m}^{(+)}+\hat{\sigma}_{x y 2 m}^{(-)}\right) \\
& F_{1 m}^{(g) z}= \frac{h_{1}}{6}\left(2 \hat{\sigma}_{z x 1 m}+\hat{\sigma}_{z x 2 m}\right) \\
& F_{i m}^{(g) x}= \frac{h_{i-1}}{6}\left(\hat{\sigma}_{x x(i-1) m}^{(+)}+2 \hat{\sigma}_{x x i m}^{(-)}\right)+ \\
& \frac{h_{i}}{6}\left(2 \hat{\sigma}_{x x i m}^{(+)}+\hat{\sigma}_{x x(i+1) m}^{(-)}\right) \\
& F_{i m}^{(g) y}= \frac{h_{i-1}}{6}\left(\hat{\sigma}_{x y(i-1) m}^{(+)}+2 \hat{\sigma}_{x y i m}^{(-)}\right)+ \\
& \frac{h_{i}}{6}\left(2 \hat{\sigma}_{x y i m}^{(+)}+\hat{\sigma}_{x y(i+1) m}^{(-)}\right) \\
& F_{i m}^{(g) z}= \frac{h_{i-1}}{6}\left(\hat{\sigma}_{z x(i-1) m}+2 \hat{\sigma}_{z x i m}\right)+ \\
& \frac{h_{i}}{6}\left(2 \hat{\sigma}_{z x i m}+\hat{\sigma}_{z x(i+1) m}\right) \quad \text { for } \quad 2 \leq i \leq N, \quad \text { for } \quad 2 \leq i \leq N \\
& F_{(N+1) m}^{(g) x}= \frac{h_{N}}{6}\left(\hat{\sigma}_{x x N m}^{(+)}+2 \hat{\sigma}_{x x(N+1) m}^{(-)}\right) \\
& F_{(N+1) m}^{(g) y}= \frac{h_{N}}{6}\left(\hat{\sigma}_{x y N m}^{(+)}+2 \hat{\sigma}_{x y(N+1) m}^{(-)}\right), \\
& F_{(N+1) m}^{(g) z}= \frac{h_{N}}{6}\left(\hat{\sigma}_{z x N m}+2 \hat{\sigma}_{z x(N+1) m}\right) . \\
&
\end{aligned}
$$

In the above, $\hat{\sigma}_{x x i m}^{(-)}$and $\hat{\sigma}_{x x i m}^{(+)}(1 \leq i \leq N+1)$ denote the $m$-th mode normal stresses in the $x$ direction just, above and below the $i$-th interface, respectively. Similarly, $\hat{\sigma}_{x y i m}^{(-)}$and $\hat{\sigma}_{x y i m}^{(+)} \quad(1 \leq i \leq N+1)$ denote the $m$-th mode shear stresses in the $y$ direction just, above and below the $i$-th interface, respectively. If the adjacent sublayers of the $i$-th interface have the same material properties, $\hat{\sigma}_{x x i m}^{(+)}$ will be equal to $\hat{\sigma}_{x x i m}^{(-)}$, and $\hat{\sigma}_{x y i m}^{(+)}$will be equal to $\hat{\sigma}_{x y i m}^{(-)}$. 